\title{
Passive Acoustic Detection of Wind Turbine In-Flow Conditions FOR ACTIVE CONTROL AND Optimization
} Final Report

\author{
National Center for Physical Acoustics \\ The University of Mississippi \\ University, MS 38677 \\ Attn: Nathan E. Murray (PI) \\ (662) 915-3190 \\ nmurray@olemiss.edu
}

DOE Award No. DE-EE0003269

U.S. Department of Energy

Golden Field Office

Golden, CO 80401

Project Coodinator: Dwight Bailey

(412) 386-4766

wilbur.bailey@netl.doe.gov

March 12, 2012 


\section{Summary}

Wind is a significant source of energy; however, the human capability to harness this great power to produce electrical energy still has many hurdles to overcome. One of these is the unpredictability of the winds in the atmospheric boundary layer (ABL). The ABL is highly turbulent in both stable and unstable conditions (based on the vertical temperature profile) and the resulting fluctuations can have a dramatic impact on wind turbine operation. Any method by which these fluctuations could be observed, estimated, or predicted could provide a benefit to the wind energy industry as a whole.

Based on the fundamental coupling of velocity fluctuations to pressure fluctuations in the nearly incompressible flow in the $\mathrm{ABL}$, this work hypothesizes that a ground-based array of infrasonic pressure transducers could be employed to estimate the vertical wind profile over a height relevant for wind turbines. To analyze this hypothesis, experiments and field deployments were conducted. Wind tunnel experiments were performed for a thick turbulent boundary layer over a neutral or heated surface. Surface-pressure and velocity probe measurements were acquired simultaneously. Two field deployments yielded surface pressure data from a 49 element array. The second field deployment at the Reese Technology Center in Lubbock, TX, also included data from a smaller aperture, 96-element, array and a 200-meter tall meteorological tower. Analysis of the data successfully demonstrated the ability to estimate the vertical velocity profile using coherence data from the pressure array. Also, dynamical systems analysis methods were successful in identifying and tracking a gust type event.

In addition to the passive acoustic profiling method, this program also investigated a rapid response Doppler SODAR system, the optimization of wind turbine blades for enhanced power with reduced aeroacoustic noise production, and the implementation of a wireless health monitoring system for wind turbine blades. Each of these other objectives was met successfully. The use of phase unwrapping applied to SODAR data was found to yield reasonable results for per-pulse measurements. A health monitoring system design analysis was able to demonstrate the ability to use a very small number of sensors to monitor blade health based on the blade's overall structural modes. Most notable was the development of a multi-objective optimization methodology that successfully yielded an aerodynamic blade

design that produces greater power output with reduced aerodynamic loading noise. This optimization method could be significant for future design work. 


\section{Table of Contents}

Summary

List of Figures $\quad$ iv

List of Tables

1 Project Goals and Accomplishments Summary 1

1.1 Summary of Proposed Tasks . . . . . . . . . . . . . . . . . . . . . . 1

1.2 Relevance of Program Objectives to Wind Energy . . . . . . . . . . . . . . . . . 1

1.3 Program Outcomes at a Glance . . . . . . . . . . . . . . . . . . . 2

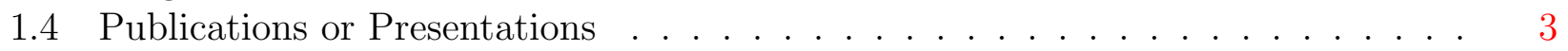

1.5 Collaborations Formed . . . . . . . . . . . . . . . . . . 3

1.6 A Note on the NCPA Infra-Sound Sensors . . . . . . . . . . . . . . . . . . . 4

2 Development of a Rapid Response Doppler SODAR 5

2.1 Summary . . . . . . . . . . . . . . . . . . . . . . 5

2.2 Task Objectives . . . . . . . . . . . . . . . . . . . . . 5

2.3 Overview of Current Doppler SODAR Measurements . . . . . . . . . . . . . 6

2.4 Procurement of ASC-3000S SODAR . . . . . . . . . . . . . . . . . . . 6

2.5 Theoretical Model of Instantaneous SODAR Signals . . . . . . . . . . . . . . . . . 7

2.6 Measurements at the WiSE Meteorological Tower . . . . . . . . . . . . . . . 16

2.7 Summary \& Conclusions . . . . . . . . . . . . . . . . . . . . . 22

3 Passive Acoustic In-Flow Profiling 27

3.1 Concept Description . . . . . . . . . . . . . . . . . . . . . . 27

3.2 Background . . . . . . . . . . . . . . . . . . . . . . . . . . . . . . . . . . . . . . . . .

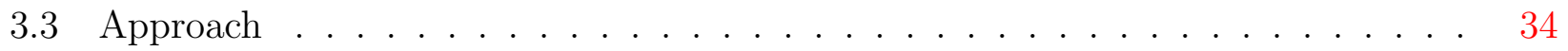

3.4 Shields' Analysis of Past Infra-Sound Data . . . . . . . . . . . . . . . . . . . 35

3.5 Details of Lab Experiments and Field Deployments . . . . . . . . . . . . . . . 36

3.6 Velocity Profile Estimation . . . . . . . . . . . . . . . . . . . . 59

3.7 Gust Detection and Tracking . . . . . . . . . . . . . . . . . . . . 70

3.8 Conclusions and Future Work . . . . . . . . . . . . . . . . . . . . . . . . 79

4 LES of Gust Generated Surface Pressure $\quad 82$

4.1 CFD Efforts of CRAFT Tech . . . . . . . . . . . . . . . . . . . . . . . . . 82

4.2 LES Simulation of the ABL . . . . . . . . . . . . . . . . . . 82

5 CFD Optimization of Rotor Blade Geometry 84 
6 Health Monitoring of Wind Turbine Systems

Appendices

A Hyperion Technology Group, Inc., IFS-3000 Infra-Sound Sensor . . A-1

B Shields' Report on Piñon Flat Data Analysis . . . . . . . . . . . B-1

C Combustion Research \& Flow Technology - Final Report . . . . . C-1

D Miltec Research \& Technology - Final Report . . . . . . . . . . . D-1 


\section{List of Figures}

Figure Number $\quad$ Page

2.1 Illustrations of a single-beam Doppler SODAR. . . . . . . . . . . . . . . 8

2.2 Diagram of a multi-beam Doppler SODAR. . . . . . . . . . . . . . . . . 9

2.3 Photo of the ASC-3000S SODAR Unit . . . . . . . . . . . . . . . . . . . . . . . . . . . . . . . . . . . . .

2.4 Simulation of Doppler Phase Wander . . . . . . . . . . . . . . . . . . 13

2.5 Simulated SODAR Echo Signal . . . . . . . . . . . . . . . . . 15

2.6 Doppler Phase versus Altitude . . . . . . . . . . . . . . . . . . 15

2.7 SODAR Return Echos from 7 Jan. 2011 . . . . . . . . . . . . . . . . 16

2.8 Unwrapped Doppler Phases for Fig. 2.7 Signals . . . . . . . . . . . . . . . . . . 17

2.9 Comparison of WiSE Tower Data to Standard SODAR Output . . . . . . . . . 18

2.10 Doppler Phase Derived SODAR Data vs. WiSE Tower Data . . . . . . . . . . . 21

2.11 Near Real-Time Doppler Phase SODAR Comparison A . . . . . . . . . . . . . . 23

2.12 Near Real-Time Doppler Phase SODAR Comparison B . . . . . . . . . . . . . . 24

2.13 Near Real-Time Doppler Phase SODAR Comparison C . . . . . . . . . . . . . . 25

3.1 Infra-Sound Wind Profiling Concept Illustration . . . . . . . . . . . . . . . . . . 28

3.2 Pressure Wave Propagating Across Array . . . . . . . . . . . . . . . . . . . . . . 29

3.3 Early Result of Shields Showing Cross-Correlation Technique . . . . . . . . . . . 30

3.4 Piñon Flat, CA, 2007 Infra-Sound Array Setup . . . . . . . . . . . . . . 36

3.5 Characteristic Wind and Pressure Data from Piñon Flat . . . . . . . . . . . . 37

3.6 Correlation-Derived Convection Velocity vs. Frequency . . . . . . . . . . . . . 37

3.7 NCPA Two-Foot Tunnel - Inlet Configuration . . . . . . . . . . . . . . . . . . 39

3.8 NCPA Two-Foot Tunnel - Microphone Installation . . . . . . . . . . . . . . . . 39

3.9 NCPA Two-Foot Tunnel - Hot-Wire Setup . . . . . . . . . . . . . . . . . . . . . 40

3.10 NCPA Two-Foot Tunnel - Hot-Wire Calibration Setup . . . . . . . . . . . . . . 41

3.11 NCPA Two-Foot Tunnel - Velocity Spectra vs. Elevation . . . . . . . . . . . . . 42

3.12 NCPA Two-Foot Tunnel - Velocity Spectra vs. Speed and Surf. Temp. . . . . . 42

3.13 NCPA Two-Foot Tunnel - Vel. Profile at Streamwise Stations . . . . . . . . . . 43

3.14 NCPA Two-Foot Tunnel - Vel. Profile vs. Surf. Temp. . . . . . . . . . . . . . . 43

3.15 NCPA Two-Foot Tunnel - Vel. Profile vs. Freestream Speed . . . . . . . . . . . 43

3.16 NCPA Two-Foot Tunnel - Turbulent Energy vs. Mean Vel. and Surf. Temp. . . 44

3.17 NCPA Two-Foot Tunnel - Characteristic Surface Pressure Spectra . . . . . . . . 44

3.18 NCPA Two-Foot Tunnel - Wall-Pressure Correlations . . . . . . . . . . . . . . . 45

3.19 Locke Station - Test Site . . . . . . . . . . . . . . . . . . . . . . . . . . . 46

3.20 Locke Station - Pressure Transducer Layout . . . . . . . . . . . . . . . . . . . 46

3.21 Locke Station - Photo of SODAR System Setup . . . . . . . . . . . . . . . . . . . 47

3.22 Locke Station - Sample SODAR Signal Returns . . . . . . . . . . . . . . . . . . 47

3.23 Locke Station - South-East View from Array Center . . . . . . . . . . . . . . . 48

3.24 Locke Station - Temperature and North-Wind Velocity . . . . . . . . . . . . . . 49

3.25 Locke Station - NCPA-UMX and MRT-TT Infrasound Sensor Units . . . . . . . 49

3.26 Locke Station - Pressure Time Traces from NCPA-UMX and MRT-TT Sensors 50 
3.27 Locke Station - Pressure PDFs . . . . . . . . . . . . . . . . . . . . 51

3.28 Locke Station - Pressure PDFs - LOG Scale . . . . . . . . . . . . . . . . . 51

3.29 Locke Station - Pressure Spectra - Midnight . . . . . . . . . . . . . . . . 52

3.30 Locke Station - Pressure Spectra - Midday . . . . . . . . . . . . . . . . . 53

3.31 Reese Technology Center (RTC) - Met. Tower . . . . . . . . . . . . . . . . 54

3.32 RTC - Met. Tower Measurements . . . . . . . . . . . . . . . . . . 55

3.33 RTC - Small Aperture Array . . . . . . . . . . . . . . . . . . . . 55

3.34 RTC - Large Aperture Array . . . . . . . . . . . . . . . . . . . . . . 56

3.35 RTC - SODAR Setup . . . . . . . . . . . . . . . . . 56

3.36 RTC - Small Aperture Array Average Pressure vs. Time . . . . . . . . . . . . 57

3.37 RTC - Prop-Anemometer Wind Speed . . . . . . . . . . . . . . . . . . 57

3.38 RTC - Sonic Anemometer Wind Speed . . . . . . . . . . . . . . . . . 57

3.39 RTC - Sonic Anemometer Wind Direction . . . . . . . . . . . . . . . 58

3.40 RTC - Prop-Anemometer Vertical Wind Speed . . . . . . . . . . . . . 58

3.41 RTC - Atmospheric Temperature . . . . . . . . . . . . . . . . . 58

3.42 RTC - Surface Pressure Spectra . . . . . . . . . . . . . . . . . . . . . . 59

3.43 Convection Velocity vs. Sensor Separation Distance . . . . . . . . . . . . . . 60

3.44 Low-Speed Tunnel - Pressure Time Scale . . . . . . . . . . . . . . . . . . . . . . 61

3.45 Low-Speed Tunnel - Length Scale . . . . . . . . . . . . . . . . . . . . . . 61

3.46 Low-Speed Tunnel - Signal Coherence . . . . . . . . . . . . . . . . . . . . . . 64

3.47 Low-Speed Tunnel - Spectra of Pressure Signals . . . . . . . . . . . . . . . . . . 64

3.48 Low-Speed Tunnel - Mic. 3-to-4 Transfer Function . . . . . . . . . . . . . . . . 65

3.49 Low-Speed Tunnel - Computed Freq. Dependent Time Lag . . . . . . . . . . . . 65

3.50 Low-Speed Tunnel - Wave Length vs. Convection Velocity . . . . . . . . . . . . 65

3.51 Low-Speed Tunnel - Estimated Mean Velocity Profile . . . . . . . . . . . . . . . 66

3.52 Low-Speed Tunnel - Est. Mean Vel. Profiles at Elevated Surface Temp. . . . . . 66

3.53 RTC Field Data - Sensor 1-to-2 Signal Coherence . . . . . . . . . . . . . . . 67

3.54 RTC Field Data - Computed Freq. Dependent Time Lag . . . . . . . . . . . . 67

3.55 RTC Field Data - Wave Length vs. Convection Velocity . . . . . . . . . . . 68

3.56 RTC Field Data - Estimated Mean Velocity Profiles _ . . . . . . . . . . . . . 69

3.57 POD Modes of ABL Surface Pressure . . . . . . . . . . . . . . . . . . 71

3.58 POD Basis Obtained from DMD Analysis . . . . . . . . . . . . . . 73

3.59 DMD Spectrum . . . . . . . . . . . . . . . . . . . 74

3.60 Evolution of Dynamic Mode 1 Showing Propagation . . . . . . . . . . . . 75

3.61 DMD Spectrum of Synthetic Disturbance . . . . . . . . . . . . . . . . . . . . . 77

3.62 Dynamic Modes of Synthetic Disturbance . . . . . . . . . . . . . . . . 77

3.63 Synthetic Disturbance Time Series Snapshots . . . . . . . . . . . . . . . 78

3.64 Synthetic Disturbance Time Series Reconstruction . . . . . . . . . . . . . . 78 


\section{List of Tables}

Table Number $\quad$ Page

1.1 Program Outcomes at a Glance . . . . . . . . . . . . . . . . . . 2 


\section{1 | Project Goals and Accomplishments Summary}

\subsection{Summary of Proposed Tasks}

At the outset, the proposed research program's primary object was to obtain real-time measurements of wind turbine in-flow conditions using an array of passive, ground-based pressure sensors. As the sensors in question operate to sense both audible and infrasound frequencies, the measurement of wind turbine noise in the infrasound region was included as an objective. A secondary objective was to obtain real-time measurements of the in-flow conditions from an active Sonic Detection and Ranging (SODAR) system. Because of the inherent coupling between atmospheric turbulence, wind turbine noise production, and the health of the turbine components, two additional objectives were pursued: a blade design optimization to produce a low-noise rotor with optimized power output, and a wireless, flush-mounted health monitoring system that could monitor the large amplitude infrasound signatures.

Based on the proposed objectives, four research tasks were defined. The chapters of this report are organized to present final reports associated with each of these tasks:

Task 1 Chapter 2: "Development of a Rapid Response Doppler SODAR"

Task 2 Chapters 3 \& 4: "Passive Acoustic In-Flow Profiling"

Task 3 Chapter 5: "CFD Optimization of Rotor Blade Geometry"

Task 4 Chapter 6: "Health Monitoring of Wind Turbine System"

\subsection{Relevance of Program Objectives to Wind Energy}

The relevance of the current work is summarized well by some comments found in a 2005 report by Kelley et al. ${ }^{[2]}$ which presents a computational analysis of the effect of large-scale turbulent structures on the aeroelastic response of wind turbines. The authors suggest that the "greatest structural fatigue damage tends to occur during the nighttime hours from coherent turbulence that develops in the stable, nocturnal atmospheric boundary layer." The observed "nocturnal low-level jet streams (LLJs) provide increased wind resource with height but also result in intense vertical wind shear and temperature gradients." The authors note that this wind shear feeds Kelvin-Helmholtz instabilities that produces billows which subsequently impact the turbines. These are exactly the type of events that the current work seeks to measure and predict using ground-based measurements.

Kelley et al. ${ }^{[2]}$ also note that the breakdown of the billow structures produces regions of high coherent turbulent kinetic energy which are smaller in size than the spatial extent of the turbine itself. This results in elastic modes being excited rather than producing torque. This points to a specific type of flow-field feature of interest. The current work seeks to understand the relationship between the ground-based pressure fluctuations and these salient events so that the turbine can be optimized and its life extended.

Further validation of the relevance of the current work was given through personal communication with NREL scientist Andrew Clifton who noted that the current state of the art in estimating the available wind resources at a given location has around a $12 \%$ margin of 


\begin{tabular}{|c|c|c|}
\hline $\begin{array}{c}\text { Task } \\
\text { Number }\end{array}$ & Task Description & Outcome Summary \\
\hline 1.1 & Evaluate SODAR & $\begin{array}{l}\text { Developed and demonstrated } \\
\text { framework for rapid response SODAR. }\end{array}$ \\
\hline 1.2 & $\begin{array}{l}\text { Downwind Extrapolation of } \\
\text { Wind Profiles }\end{array}$ & $\begin{array}{l}\text { No work performed - determined to } \\
\text { be out of the scope of the program. }\end{array}$ \\
\hline 2.1 & $\begin{array}{l}\text { Develop Velocity Profile } \\
\text { Prediction Methodology }\end{array}$ & $\begin{array}{l}\text { Successful demonstration of DMD } \\
\text { method for gust detection. Successful } \\
\text { demonstration of coherence method } \\
\text { for mean in-flow profile prediction. }\end{array}$ \\
\hline 2.2 & $\begin{array}{l}\text { Perform Experiments to } \\
\text { Measure Correlation }\end{array}$ & $\begin{array}{l}\text { Completed } 2 \text { field experiments. } \\
\text { Collaboration with TTU resulted in } \\
\text { synchronous tower meteorology and } \\
\text { ground-based pressure measurements. }\end{array}$ \\
\hline 3.1 & $\begin{array}{l}\text { Framework Development for } \\
\text { Design Optimization }\end{array}$ & $\begin{array}{l}\text { Framework implementation } \\
\text { completed. }\end{array}$ \\
\hline 3.2 & $\begin{array}{l}\text { Improvements to Noise } \\
\text { Prediction }\end{array}$ & $\begin{array}{l}\text { Successfully completed and } \\
\text { implemented in optimizer. }\end{array}$ \\
\hline 3.3 & $\begin{array}{l}\text { Prediction of Wind Tunnel } \\
\text { Boundary Layer }\end{array}$ & $\begin{array}{l}\text { Preliminary computations complete. } \\
\text { No conclusive evidence of gust-like } \\
\text { behavior. }\end{array}$ \\
\hline 3.4 & $\begin{array}{l}\text { Integration of Aero-Elastic } \\
\text { Effects in CFD Framework }\end{array}$ & Contingent on future funding. \\
\hline 4.1 & $\begin{array}{l}\text { Development of Wireless Sensor } \\
\text { Technology }\end{array}$ & $\begin{array}{l}\text { Subcontractor focused on data } \\
\text { analysis of fictitious system. No sensor } \\
\text { development was conducted. }\end{array}$ \\
\hline 4.2 & $\begin{array}{l}\text { Apply Sensor Technology to } \\
\text { Working Wind Turbine }\end{array}$ & $\begin{array}{l}\text { Subcontractor did not pursue } \\
\text { implementation. }\end{array}$ \\
\hline
\end{tabular}

Tab. 1.1. Summary of sub-task items and associated outcomes.

error. Clifton noted that the ability to provide ground based estimation of the wind resource, even if just to reduce the margin of error by $3-4 \%$, would be a "game changing technology for the industry. ${ }^{[1]}$ "

\subsection{Program Outcomes at a Glance}

Table 1.1 shows at a glance the sub-task items and summarizes the associated outcomes. Greater detail on the task outcomes is presented in the corresponding chapters.

\subsubsection{Comments on Program Management}

In October 2010, the principle investigator (PI), Dr. John M. Seiner, passed away unexpectedly. In his absence, the lead researcher for Task 2, Dr. Nathan E. Murray, assumed leadership as the PI with Dr. Raspet and Dr. Gilbert remaining as Co-PIs. Dr. Murray remained the technical lead for Task 2. Dr. Gilbert continued as the technical lead for Task 1. Dr. Raspet continued as the principle mentor for graduate students working on related research. 
Tasks 3 and 4 were subcontracted out to research partners. Combustion Research and Flow Technology, Inc. (CRAFT-Tech) were subcontracted to complete Task 3. Task 4 was subcontracted to Miltec Research and Technology, Inc. (MR\&T) of Oxford, MS. The subcontract to MR\&T also included support for the field experiments performed in Task 2.

An additional subcontract was let to retain the consulting services of Dr. F. D. Shields who has significant experience in performing infrasound field experiments and analyzing the related data. Dr. Shields completed data analysis in support of Task 2.

\subsection{Publications or Presentations}

Murray, N. (2011) "Measurements of Atmospheric Boundary Layer Related Surface Pressure Fluctuations." $8^{\text {th }}$ Meeting of the Midsouth Chapter of the Acoustical Society of America. Oxford, MS. April, 15-16.

Lyons, G. \& Murray, N. (2011) "Description of Coherent Features in the Atmospheric Boundary Layer by Low-Dimensional Analysis of Surface Pressures." Abstract D1.00006, 64 ${ }^{\text {th }}$ Annual Meeting of the APS Division of Fluid Dynamics., November 20-22, Baltimore, MD.

ABSTRACT: Short-term localized forecasting of the near-earth atmospheric boundary layer could enhance empirical models and provide input for wind energy control systems. It is expected that a successful forecasting method could be developed through characterization of the coherent turbulent structures in the near-earth flow field. The coherence of these features suggests description by a low-dimensional method, such as the dynamic mode decomposition. To this end, a circular array of pressure transducers approximately 60 meters in diameter was deployed on the earths surface, and time series were recorded at each sensor location. As scalar observables, these pressure measurements are functions of the flow field system. As such, by decomposing these data into dynamic modes, the spatial and temporal coherence of local features in the near-earth atmospheric boundary layer is described.

Publication of the CRAFT Tech optimization efforts and the Reese Technology Center field deployment are anticipated.

\subsection{Collaborations Formed}

A number of informal collaborations were formed during this program. To date, none of these have resulted in funded collaborative efforts; however, discussions with the TTU Wind Science and Engineering Center to have NCPA provide acoustic support for their research efforts have been positive.

- Sandia National Laboratories: Dr. Joshua Paquette provided NCPA with FEA models of the CX-100 and WindPACT turbine blades for the health monitoring analysis.

- U. of Wyoming, Wind Energy Research Center: A letter of support was provided to Dr. Jonathan Naughton to identify a proposal of his on stochastic estimation of inflow conditions as relevant to the current work. 
- Texas Tech University, Wind Science and Engineering Center: A research agreement was put in place to allow NCPA to conduct the field deployment at the Reese Technology Center. TTU provided data acquisition support to NCPA and provided the data from the 200-meter meteorological tower during the field deployment. The successful experimental campaign has led to further discussions that may lead to additional collaborative work.

\subsection{A Note on the NCPA Infra-Sound Sensors}

At the time of this program, the NCPA-UMX infra-sound sensor was still in a developmental phase with ongoing improvement occurring during the period of performance. Therefore, the NCPA-UMX sensors used in the two field deployments discussed in Chapter 3 had slight differences primarily in the digitizer and data acquisition electronics. As of the conclusion of this program, the NCPA-UMX sensor has been commercialized and is available through Hyperion Technology Group, Inc. Details of the analog version of the sensor (to be used with the customers own digitizer and acquisition system) are attached as Appendix A. Hyperion does offer its own digitizer providing a complete stand alone wireless sensor unit, but the frequency response characteristics are unchanged from the analog unit presented in the appendix.

\section{Bibliography}

[1] Clifton, A. (2012) personal communication.

${ }^{[2]}$ Kelley, N. D.; Jonkman, B. J.; Scott, G. N.; Bialasiewicz, J. T.; \& Redmond, L. S. (2005) "The Impact of Coherent Turbulence on Wind Turbine Aeroelastic Response and Its Simulation." National Renewable Energy Laboratory, Conference Paper NREL/CP500-38074. 


\section{2 | Development of a Rapid Response Doppler SODAR}

\subsection{Summary}

A theoretical simulation model has been developed to simulate the instantaneous wave form for the echoes received by a Doppler SODAR. The model led to a new approach for processing SODAR signals which uses the unwrapped Doppler phase. The new approach, denoted as "Doppler phase method," was applied to near real-time data collected during a 24-hour measurement period with an Atmospheric Systems Corporations (ASC) Doppler SODAR. Wind speeds were estimated every 13.6 seconds using the Doppler phase method at four heights $(47 \mathrm{~m}, 75 \mathrm{~m}, 116 \mathrm{~m}$, and $158 \mathrm{~m})$, and compared to the corresponding sonic anemometer measurements on a meteorological tower. The tower/SODAR comparison clearly demonstrates the ability of the Doppler SODAR to track rapid changes in wind speed. For example, a wind speed increase from $2 \mathrm{~m} / \mathrm{s}$ to nearly $10 \mathrm{~m} / \mathrm{s}$ in less than two minutes was accurately tracked. However, in general, the variance in the SODAR wind speed estimates was larger than desired. In an effort to reduce the variance, a simple Kalman filter was developed to track the wind speed in conjunction with the Doppler phase method. The simple Kalman filter did not reduce the variance, but did demonstrate the general applicability of Kalman filter algorithms to estimating wind speed profiles from Doppler SODAR echoes. Since Kalman filter methodology is already highly developed, developing a high-performance, adaptive Kalman filter is proposed for follow-on research.

\subsection{Task Objectives}

\subsubsection{Objective - SODAR Determined Wind Velocity Profile}

Task 1.1 Evaluate the potential of SODAR to estimate real-time or near real-time vector wind profiles: (a) review existing SODAR data comparisons with anemometer data from meteorological towers, (b) use Large Eddy Simulation (LES) model to study the evolution of a wind profile as it undergoes downwind advection, and (c) acquire unprocessed and processed SODAR data and investigate pulse-to-pulse variability in the estimated wind profile to determine the extent to which the estimated profiles meet physical criteria (vector velocity continuity, zero divergence). Field data collection will likely take place at a national lab that is to be determined.

Task 1.2 Conduct tests on measurement and downwind extrapolation of wind profiles: (a) lease or buy 2 SODARs that can be configured for research measurements (alternatively, modify an existing NCPA Remtec SODAR for research use as the second SODAR), (b) compare profiles measured at a fixed location with those measured at increasing downwind distances to determine the decay length/time of the long-period components of the advected wind profile and investigate empirically the capability of a single SODAR to estimate the downwind decay of the long-period components of the wind profile, (c) use the SODAR data to begin to develop and test dynamic estimation models for measuring and extrapolating wind profiles from a fixed upwind location to 
a downwind location with the objective to estimate the turbine inflow at hub height from an upwind wind profile measurement, and (d) carry out a full-scale test with an instrumented wind turbine. The site for this test will likely be at a national lab. Instrumentation will include two commercial SODARs along with a tethersonde and/or radiosonde for meteorological data collection.

\subsubsection{Tasks Addressed}

Early in the present investigation, it became apparent that essentially no experimental or theoretical information existed on real-time or near real-time wind speed profiles determined by Doppler SODARs. As a result, considerable effort had to be devoted to developing a credible theoretical model in order to begin the analysis of real-time SODAR data. Consequently no research, theoretical or experimental, was conducted on the downwind evolution of wind speed profiles. The investigation focused entirely on understanding the real-time, pulse-to-pulse SODAR echoes and comparing the near real-time wind speed estimates with ground-truth data collected on a 200-m instrumented meteorological tower at Texas Tech University.

\subsection{Overview of Current Doppler SODAR Measurements}

SODAR (SOund Detection And Ranging) systems are used to remotely sense the structure of the lower layer of the atmosphere (up to several hundred meters). The measurement of wind speed by Doppler SODAR has a considerable history going back several decades. During the past 30 years, Doppler SODAR data have been extensively compared with tower anemometer measurements. ${ }^{[1,3,6]}$ Typically, when SODAR measurements are averaged for 10-15 minutes the agreement with tower anemometers has been shown to be fairly good, having a standard deviation of approximately $1 \mathrm{~m} / \mathrm{s}^{[3]}$ In contrast, for short-time (i.e. not time averaged) wind speed measurements, there appear to be no studies comparing SODAR measurements to anemometer measurements. This is unfortunate since monitoring rapid changes in wind speed is an important application for the wind turbine industry. The absence of any existing short-time SODAR/anemometer comparisons motivated the investigation reported here. This report presents a theoretical analysis that is applied to data from a 2011 field experiment aimed at assessing the capability of a Doppler SODAR to continuously monitor rapid changes in wind speed at altitudes from $47 \mathrm{~m}$ to $158 \mathrm{~m}$.

\subsection{Procurement and Testing of an ASC-3000S SODAR}

The wind speed profiles output by typical commercially available SODARs are usually averaged over 1 - 20 minutes. For the present investigation, however, access to the pulse-bypulse output of the SODAR was essential. Since the standard signal processing in commercial SODARs generally does not allow users access to the raw SODAR signal, a vendor was sought who would be willing to provide the proprietary information needed to access and record the real-time (pulse-by-pulse) data. Dr. Kenneth Underwood, owner of Atmospheric Systems Corporation (ASC), agreed to do so. Dr. Underwood obtained his Ph.D. in meteorology from Penn State University on the topic of SODAR design. He is recognized internationally as a 
leading SODAR expert. It was felt that Dr. Underwood and ASC would be good partners for the envisioned research. That has proved to be the case.

A model 3000S ASC SODAR was procured in the Spring of 2010, and delivery was taken in August of 2010. Two NCPA engineers were sent to the ASC office in Santa Clarita, CA, for training on the use of the model 3000S SODAR. During September 2010, they loaded the software and learned to set up and use the SODAR. Preliminary testing took place in October and November at the NCPA Locke Station, MS, experiment site. Preliminary comparisons were made with cup anemometers on a tethersonde, which proved to be satisfactory.

Since the Locke Station site is not secure, it could not be used for long-term testing. Consequently, in November 2010, the SODAR was taken to a secure site at the University of Mississippi Biological Field Station. The SODAR remained at the Biological Field Station while long-term data were collected and analyzed through the winter of 2010 and into the Spring of 2011. In May 2011, the SODAR was brought back to be prepared for a comparison with data from a 200-m instrumented meteorological tower during a field test conducted in June 2011 at Texas Tech University. The analysis of the data from the Texas Tech field test is reported here along with the theoretical model on which the analysis is based.

\subsection{A Theoretical Model for Instantaneous SODAR Signals}

While testing and evaluation of the ASC SODAR at the Biological Field Station were being done in the Spring of 2011, research was simultaneously being conducted to develop a theoretical model for the instantaneous wave form (amplitude and phase versus time). It was felt that, in order to effectively analyze the data from the Biological Field Station, more insight was needed on the basic physical processes that govern the instantaneous waveforms of the received echoes.

While there is a good deal of information on the average backscattered acoustic power measured by SODARs, no experimental or theoretical information could be found on predicting the instantaneous waveform, which is essential for real-time wind speed estimation. Hence it became imperative to conduct a fundamental theoretical study aimed at understanding the basic physical mechanisms in real-time acoustic backscatter from turbulence. This investigation is apparently the first study aimed at such an understanding.

\subsubsection{The Physical Principles of Doppler SODARs}

Fig. 2.1 shows (schematically) acoustic backscatter from atmospheric turbulence. An array of transducers is used to project an acoustic pulse vertically in a beam. Turbulencegenerated inhomogeneities in temperature create sound speed fluctuations that scatter sound and lead to weak but measurable echoes. The transducers, which also act as receivers, detect the faint echoes, which are recorded and processed to determine the Doppler shift.

By electronically adjusting the phase of the transducer array, the SODAR can project three acoustic beams as shown in Fig. 2.2, generally in sequence. The $u$-beam is in the $x-z$ plane, the $v$-beam is in the $y-z$ plane, and $w$-beam points directly upward. The Doppler shifts on the three beams determine the $u$-component ( $x$-direction), $v$-component ( $y$-direction), and $w$-component ( $z$-direction), of the vector wind velocity. Fig. 2.3 is a photograph of the model 3000S ASC SODAR actually used in the investigation. From left 


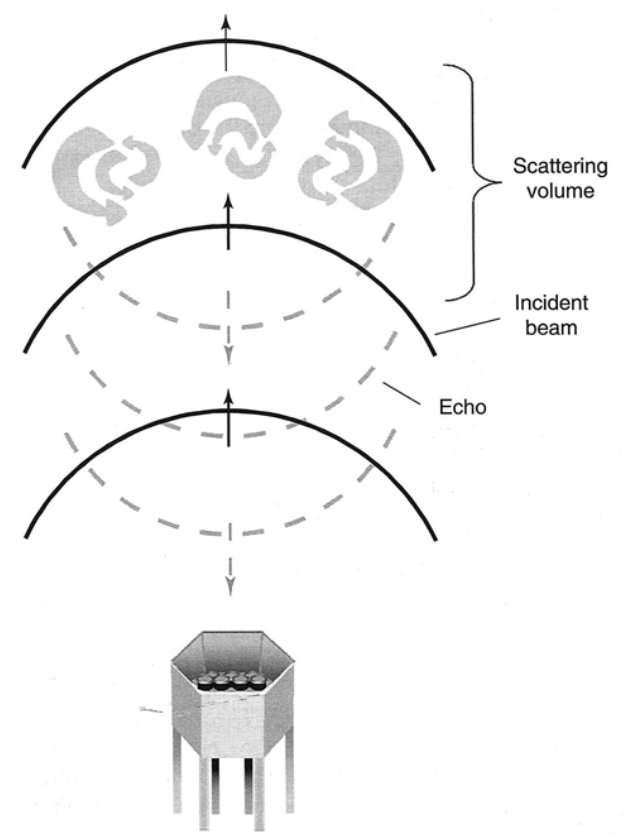

Fig. 2.1. Illustrations of a single-beam Doppler SODAR.

to right in Fig. 2.3 are NCPA researchers Mr. Rick Burgett, Mr. Rommel Stribling, and Dr. Garth Frazier.

The mean flow of the turbulent atmosphere produces a Doppler shift on each of the SODAR beams, so that together the full three-dimensional vector wind velocity can be estimated. Note, however, that even at one instant of time, due to the sampling volume of the SODAR, the measurement yields a vector wind velocity averaged over a considerable volume of atmosphere. For example, for the speed of sound $c_{0}=340 \mathrm{~m} / \mathrm{s}$ and a SODAR pulse of length $\tau=0.2 \mathrm{~s}$, the vertical extent of the pulse is $c_{0} \tau=68 \mathrm{~m}$. At any instant of time, the received echo comes from a sampling volume with a vertical extent of $c_{0} \tau / 2=34 \mathrm{~m}$. With a $6^{\circ}$ beam $(\approx 0.1 \mathrm{rad})$, the averaging volume at $100 \mathrm{~m}$ altitude is thus approximately $3400 \mathrm{~m}^{3}$. Hence, even before any temporal averaging, the SODAR wind speed estimate is an average wind speed that represents the mean wind velocity in a large volume of the atmosphere.

The geometry of the SODAR beams in most modern phased-array SODARs is similar to that shown in Fig. 2.2. For example, for the ASC SODAR the $u$-beam is in the $x-z$ plane and makes an angle of $21^{\circ}$ with the $z$-axis. Similarly, the $v$-beam is in the $y-z$ plane and also makes an angle of $21^{\circ}$ with the $z$-axis. The $w$-beam points along the $z$-axis (i.e., directly upward).

On a given beam, the Doppler shift, $\Delta f$, is given by $\left(2 v_{\text {proj }} / c_{0}\right) f_{0}$, where $v_{\text {proj }}$ is the projection of the mean wind onto the beam direction and is given by $v_{p r o j}=\boldsymbol{v} \cdot \hat{\boldsymbol{n}}$ where $\boldsymbol{v}$ is the volume average of the vector wind velocity in the sampling volume, and $\hat{\boldsymbol{n}}$ is a unit vector pointing in the direction of the beam. The quantity $f_{0}$ is the frequency of the SODAR, which for the ASC SODAR is $2800 \mathrm{~Hz}$. For the $u$-component of the mean wind 


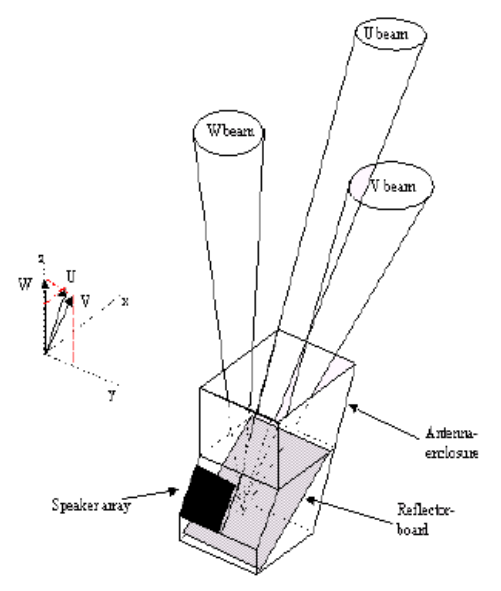

Fig. 2.2. Diagram of a multi-beam Doppler SODAR.

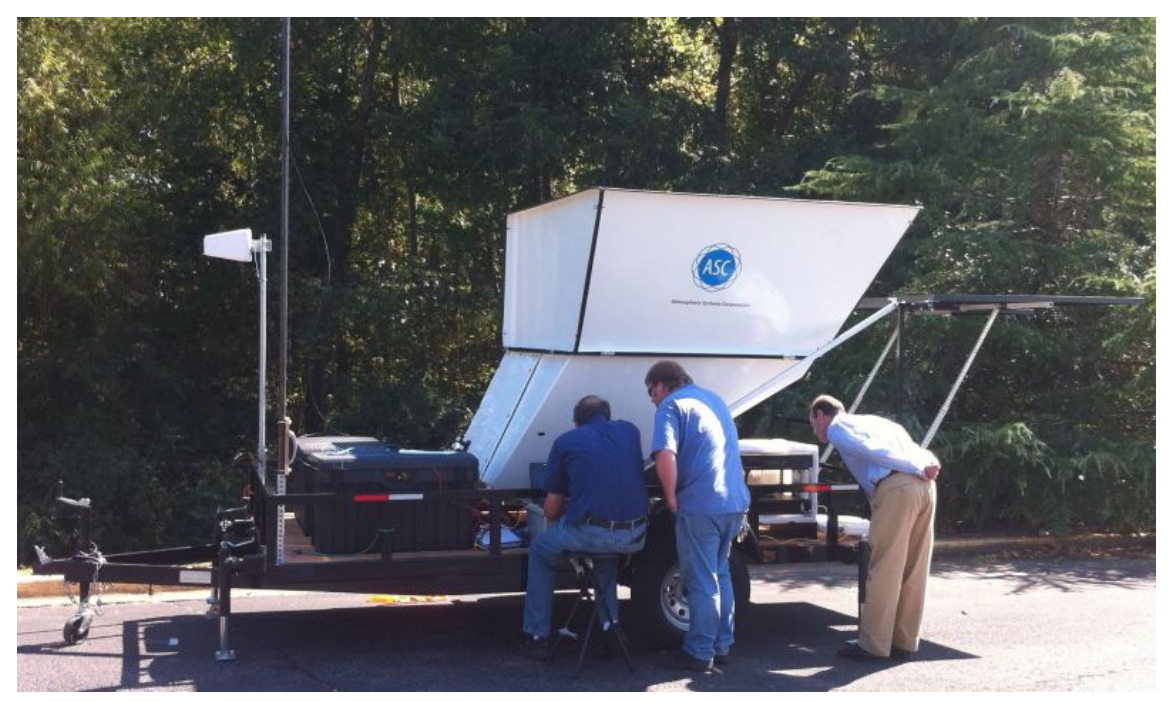

Fig. 2.3. The Atmospheric Systems Corporation multi-beam Doppler SODAR used in the present investigation. 
velocity (x-component), the projection $v_{\text {proj }}=u \sin \theta$, where $\theta=21^{\circ}$ is the tilt angle on the ASC SODAR. Similarly for the $v$-beam, $v_{\text {proj }}=v \sin \theta$. For the $w$-beam, $v_{\text {proj }}=w$.

As will be discussed in detail later, $\Delta f$ can be determined either from the Fourier transform of the backscattered signal on a given beam or by the time derivative for the unwrapped phase of the signal. Once values of $\Delta f$ are obtained for each beam, $v_{\text {proj }}=\left(c_{0} / 2\right)\left(\Delta f / f_{0}\right)$ is determined for each beam. Hence we have $u=\left(c_{0} / 2 \sin \theta\right)\left(\Delta f_{u} / f_{0}\right), v=\left(c_{0} / 2 \sin \theta\right)\left(\Delta f_{v} / f_{0}\right)$, and $w=\left(c_{0} / 2\right)\left(\Delta f_{w} / f_{0}\right)$, where the subscript on $\Delta f$ indicates the beam. The mean horizontal wind speed is then given by $s=\sqrt{u^{2}+v^{2}}$. The vertical component $w$ is typically an order of magnitude smaller than $u$ and $v$, and is generally neglected. The quantity $w$ is not considered in the analysis given in this report.

As indicated above, to determine the real-time values of the horizontal wind, the critical quantities to be measured on a SODAR are the real-time Doppler shifts on the $u$ and $v$ beams. Accurately estimating these Doppler shifts is the focus of the present analysis. We present what to our knowledge is a new method for obtaining the Doppler shift using the "unwrapped" phase on the $u$ beam and the $v$ beam.

\subsubsection{Estimating Acoustic Scattering from "Frozen Turbulence"}

The Doppler shift on a given SODAR beam is modeled here as the sum of two components: a random unpredictable component and a systematic, deterministic component determined by the mean wind flow. The deterministic component is straightforward to estimate. The random component, in contrast, requires a theoretical model that captures the essential physics but is simple enough for a useful analysis.

\subsubsection{Gaussian Statistics of the Impulse Response}

To estimate the random component, a first-order Born approximation ${ }^{[5]}$ is used to compute backscatter for an acoustic Dirac delta function pulse scattering from a "snapshot" of turbulence with no mean flow. Such turbulence is here referred to as "frozen" turbulence. It is argued that the central limit theorem leads to a Gaussian statistical distribution for an ensemble of many backscatter snapshots from frozen turbulence.

The integral equation for the full Green's function (point source solution) for an acoustic point source of angular frequency $\omega$ in an atmosphere with sound speed $c_{0}$ is given by

$$
G\left(\mathbf{R}, \mathbf{R}^{\prime}\right)=e^{-i \omega t} G_{0}\left(\mathbf{R}, \mathbf{R}^{\prime}\right)+\frac{e^{-i \omega t}}{4 \pi} \int G_{0}\left(\mathbf{R}, \mathbf{R}^{\prime \prime}\right) \delta k^{2}\left(\mathbf{R}^{\prime \prime}\right) G\left(\mathbf{R}^{\prime \prime}, \mathbf{R}^{\prime}\right) d \mathbf{R}^{\prime \prime},
$$

where $G_{0}\left(\mathbf{R}, \mathbf{R}^{\prime}\right)=\exp \left(i k_{0}\left|\mathbf{R}-\mathbf{R}^{\prime}\right|\right) /\left|\mathbf{R}-\mathbf{R}^{\prime}\right|, \delta k^{2}\left(\mathbf{R}^{\prime \prime}\right)=k^{2}\left(\mathbf{R}^{\prime \prime}\right)-k_{0}^{2}$, and $k_{0}=\omega / c_{0}$. The term $G_{0}\left(\mathbf{R}, \mathbf{R}^{\prime}\right)$ on the right-hand side is the unscattered field and the second term is the scattered field.

Since acoustic backscatter from turbulence is very weak the first-order Born approximation holds which assumes $G\left(\mathbf{R}, \mathbf{R}^{\prime}\right) \approx G_{0}\left(\mathbf{R}, \mathbf{R}^{\prime}\right)$ on the right-hand side, so that the scattered field, $\delta G$, due to $\delta k^{2}$ is given by

$$
\delta G \approx \frac{e^{-i \omega t}}{4 \pi} \int G_{0}\left(\mathbf{R}, \mathbf{R}^{\prime \prime}\right) \delta k^{2}\left(\mathbf{R}^{\prime \prime}\right) G_{0}\left(\mathbf{R}^{\prime \prime}, \mathbf{R}^{\prime}\right) d \mathbf{R}^{\prime \prime} .
$$


The quantity $\delta k^{2} \approx-2\left(\delta c / c_{0}\right) k_{0}^{2}$, where $\delta c\left(\mathbf{R}^{\prime \prime}\right)=c\left(\mathbf{R}^{\prime \prime}\right)-c_{0}$ is the sound speed fluctuation generated by turbulent temperature fluctuations. This notation yields

$$
\delta G \approx \frac{-2 k_{0}^{2} e^{-i \omega t}}{4 \pi c_{0}} \int G_{0}\left(\mathbf{R}, \mathbf{R}^{\prime \prime}\right) \delta c\left(\mathbf{R}^{\prime \prime}\right) G_{0}\left(\mathbf{R}^{\prime \prime}, \mathbf{R}^{\prime}\right) d \mathbf{R}^{\prime \prime} .
$$

Now, the impulse response for backscatter received at $\mathbf{R}=\mathbf{R}^{\prime}=0$, denoted as $\sigma(t)$, is given by the integral of $\delta G$ over frequency (a Dirac delta function acoustic pulse has infinite bandwidth):

$$
\begin{aligned}
\sigma(t) & \approx \frac{-1}{2 \pi c_{0}^{3}} \iint \omega^{2} e^{-i \omega t} G_{0}\left(0, \mathbf{R}^{\prime \prime}\right) \delta c\left(\mathbf{R}^{\prime \prime}\right) G_{0}\left(\mathbf{R}^{\prime \prime}, 0\right) d \mathbf{R}^{\prime \prime} \frac{d \omega}{2 \pi}, \\
& =\frac{1}{2 \pi c_{0}^{3}} \frac{\partial^{2}}{\partial t^{2}} \iint \frac{e^{i \omega\left(2 R / c_{0}-t\right)}}{R^{\prime 2}} \delta c\left(\mathbf{R}^{\prime \prime}\right) d \mathbf{R}^{\prime \prime} \frac{d \omega}{2 \pi} .
\end{aligned}
$$

The integral over $\omega$ yields a Dirac delta function, $\delta\left(2 R^{\prime \prime} / c_{0}-t\right)=\left(c_{0} / 2\right) \delta\left(R^{\prime \prime}-c_{0} t / 2\right)$. Therefore,

$$
\sigma(t)=\frac{1}{4 \pi c_{0}^{2}} \frac{\partial^{2}}{\partial t^{2}} \iint \frac{1}{R^{2}} \delta\left(R-c_{0} t / 2\right) \delta c(R) R^{2} d R d \Omega,
$$

where $d \Omega=\sin (\phi) d \phi d \theta$ indicates the differential solid angle. It is assumed that the wave amplitude is zero outside of a solid angle, $\Delta \Omega$, and that the average over the solid angle $\Delta \Omega$ is denoted by \langle\rangle . Then (2.5) becomes

$$
\sigma(t)=\frac{\Delta \Omega}{4 \pi c_{0}^{2}}\left\langle\frac{\partial^{2}}{\partial t^{2}} \delta c\left(c_{0} t / 2\right)\right\rangle .
$$

Since the distance to the scattering surface is $R=c_{0} t / 2$, the time derivative can be written as $\partial^{2} / \partial t^{2}=\left(c_{0}^{2} / 4\right) \partial^{2} / \partial R^{2}$. Thus the impulse response is found to be

$$
\sigma(t)=\frac{\Delta \Omega}{16 \pi}\left\langle\frac{\partial^{2}}{\partial R^{2}} \delta c\left(c_{0} t / 2\right)\right\rangle .
$$

Eq. (2.7) represents the sum of many random contributions over a surface of area $\Delta \Omega R^{2}$, where $\Delta \Omega$ is of order 0.01 and $R^{2}$ is of order $10^{4} \mathrm{~m}^{2}$. Thus the sampling area is of order $100 \mathrm{~m}^{2}$. With such a large sampling area, the central limit theorem implies that $\sigma(t)$ is a random variable with a Gaussian distribution. The assumption of Gaussian statistics for $\sigma(t)$ is made in this report.

In (2.7), $\delta c$ is the turbulence-induced sound speed fluctuation, and \langle\rangle indicates the average over the scattering surface (area approximately $\Delta \Omega R^{2}$ ) that generates the SODAR signal received at time $t$. The scattering surface is at a vertical distance given by $R(t)=c_{0} t / 2$ where $c_{0}$ is the mean speed of sound, e.g., $340 \mathrm{~m} / \mathrm{s}$. The solid angle of the beam is $\Delta \Omega$ and $R$ is the distance along the beam. As noted above, a scattering surface of area $100 \mathrm{~m}^{2}$ is typical.

It is important to note in (2.7) that, because of the large size of the scattering surface, the signal $\sigma(t)$ is proportional to the average of a large number of random turbulent components. Hence, by the central limit theorem, we would expect $\sigma(t)$ to be a random variable with a Gaussian distribution ${ }^{[2,4]}$. The Gaussian assumption is made throughout the present analysis. As will be shown, in a moving medium, the volume-averaged (mean) Doppler shifts are essentially independent of the details of the Gaussian distribution. 


\subsubsection{Rayleigh Statistics of the Response to a Gated Sine Wave}

If a SODAR outputs a signal $S_{0}(t)$, then the received signal $S(t)$ on a given beam is the convolution of the output signal with the impulse response. That is,

$$
S(t)=\int S_{0}\left(t-t^{\prime}\right) \sigma\left(t^{\prime}\right) d t^{\prime} .
$$

If $S_{0}(t)$ is a gated sine wave of angular frequency $\omega_{0}$ and length $\tau$, as it is in the ASC SODAR, we have

$$
S(t)=\Re \int_{t}^{t+\tau} e^{-i \omega_{0}\left(t-t^{\prime}\right)} \sigma\left(t^{\prime}\right) d t^{\prime} .
$$

Hence the analytic signal with the carrier wave removed, denoted $S_{A}(t)$, is given by

$$
S_{A}(t)=\int_{t}^{t+\tau} e^{i \omega_{0} t^{\prime}} \sigma\left(t^{\prime}\right) d t^{\prime}
$$

It is well known that the Fourier transform of a random variable with a Gaussian distribution leads to a distribution in the complex plane where the phase is random and the amplitude is Rayleigh distributed..$^{[2]}$ In terms of the real and imaginary components of the analytic signal, both have Gaussian distributions. Hence, in the absence of mean flow in the atmosphere, the analytic signal performs a random walk in the complex plane and the phase associated with the random walk represents the random component of the Doppler shift. This phase is not a real Doppler shift, but is an unavoidable consequence of attempting to extract a Doppler shift using backscattering from a moving random medium (i.e., from turbulence). By examining how the phase evolves over time we can investigate the "phase noise" that we always must deal with in analyzing instantaneous SODAR signals. Extensive simulations were carried out for "frozen" turbulence (no mean flow) and it was found that the magnitude of the random phase variation was surprisingly small relative to expected mean Doppler shifts produced by the mean wind flow. Fig. 2.4(a) shows an example of a typical simulation of the random component of Doppler phase. In the figure the phase is plotted as a function of height instead of time, where the height is given by $c_{0} t / 2$. Note that the random wander in phase is in the range of 10 radians. In comparison, for typical Doppler shifts (greater than approximately $30 \mathrm{~Hz}$ ) the phase shifts due to mean flow are typical more than 100 radians, an order of magnitude larger than the random component. In Fig. 2.4(b) the strength of the turbulence was increased by a factor of 100, but the phase wander is not any larger. Although the strength of the turbulence is critical in governing the strength of the backscatter (and hence the signal to noise ratio), it apparently has little effect on the phase wander.

It can be concluded from Fig. 2.4 that the total Doppler phase should be reasonably stable, even with random scattering from turbulence. To further test the hypothesis, simulations were done for scattering from turbulence including mean flow. These simulations are discussed next.

\subsubsection{Acoustic Scattering from Turbulence with Mean Horizontal Flow}

In the presence of a mean flow vector $\boldsymbol{v}$, the response on a given beam is similar to (2.10), but is modified by a pulse compression/expansion factor given by $\gamma=\left(c_{0}-v_{\text {proj }}\right) /\left(c_{0}+\right.$ 


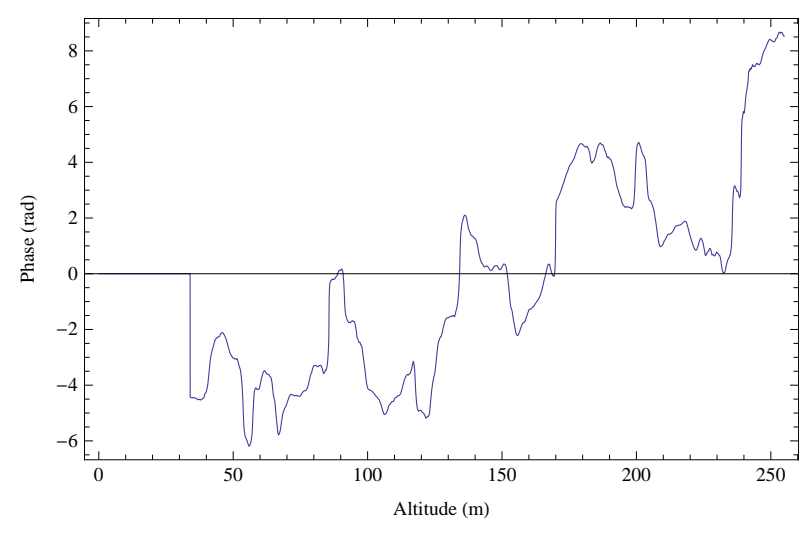

(a) Unit Turbulence Strength

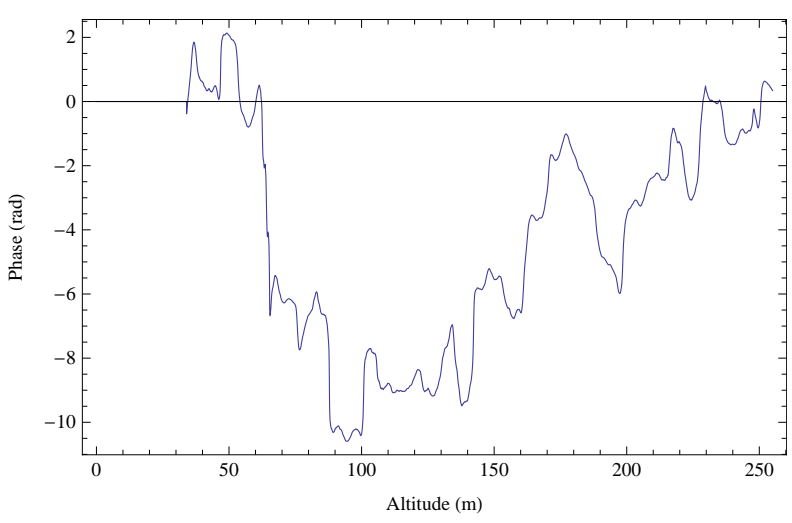

(b) Turbulence Strength $\times 100$

Fig. 2.4. Numerical simulation of the Doppler phase wander produced by scattering from turbulence.

$\left.v_{\text {proj }}\right) \approx 1-2 v_{\text {proj }} / c_{0}$, where $c_{0}$ is the mean speed of sound and $v_{\text {proj }}$ is the projection of the mean flow vector $\boldsymbol{v}$ onto the beam direction.

With mean flow, the response to an output signal $S_{0}$ is given by

$$
S(t)=\int S_{0}\left[\gamma\left(t^{\prime}\right)\left(t-t^{\prime}\right)\right] \sigma\left(t^{\prime}\right) d t^{\prime}
$$

When the flow projection is positive (beam and flow components in same direction) the pulse is stretched and the frequency is lowered (down Doppler). When it is negative (beam and flow components in opposite directions), the pulse is compressed and the frequency is raised (up Doppler).

In the case of a gated sine wave, the received signal (echo) is given by

$$
S(t)=\Re \int_{t}^{t+\tau} e^{-i \gamma\left(t^{\prime}\right) \omega_{0}\left(t-t^{\prime}\right)} \sigma\left(t^{\prime}\right) d t^{\prime}
$$

Since $v_{\text {proj }} / c \ll 1$, it is convenient to to define $2 v_{\text {proj }} / c$ as $\epsilon$ and write $\gamma=1-\epsilon$. With this notation, the analytic signal $S_{A}$ with the carrier wave removed can be written as

$$
S_{A}(t)=\int_{t}^{t+\tau} e^{i \omega_{0} \epsilon\left(t^{\prime}\right) t} e^{i \gamma\left(t^{\prime}\right) \omega_{0} t^{\prime}} \sigma\left(t^{\prime}\right) d t^{\prime} .
$$

The Dopper shift in the received signal is contained in the phase of the factor $\exp \left[i \omega_{0} \epsilon\left(t^{\prime}\right)\right]$. To simplify the integral, we add and subtract the mean phase $\bar{\phi}(t)$, which is defined as

$$
\begin{aligned}
\bar{\phi}(t) & =\omega_{0} \int_{0}^{t} \epsilon\left(t^{\prime}\right) d t^{\prime} \\
& =\left(2 \omega_{0} / c_{0}\right) \int_{0}^{t} v_{p r o j}\left(t^{\prime}\right) d t^{\prime}
\end{aligned}
$$

This leads to the result

$$
S_{A}(t)=e^{i \bar{\phi}(t)} \int_{t}^{t+\tau} e^{i\left[\omega_{0} \epsilon\left(t^{\prime}\right) t-\bar{\phi}(t)\right]} e^{i \gamma\left(t^{\prime}\right) \omega_{0} t^{\prime}} \sigma\left(t^{\prime}\right) d t^{\prime} .
$$


Since we are interested in the wind speed as a function of height and not time, the integral in (2.14) can be rewritten as an integral over $R$ where $R=c_{0} t / 2$. The mean phase then becomes

$$
\bar{\phi}(R)=\left(4 \omega_{0} / c_{0}^{2}\right) \int_{0}^{R} v_{p r o j}\left(R^{\prime}\right) d R^{\prime}
$$

Then we have for $S_{A}(R)$

$$
S_{A}(R)=e^{i \bar{\phi}(R)} \int_{c_{0} t / 2}^{c_{0}(t / 2+\tau / 2)} e^{i\left[\left(2 \omega_{0} / c_{0}\right) \epsilon\left(R^{\prime}\right) R-\bar{\phi}(R)\right]} e^{2 i\left(\omega_{0} / c_{0}\right) \gamma\left(R^{\prime}\right) R^{\prime}} \sigma\left(r^{\prime}\right) d R^{\prime} .
$$

The factor $\gamma\left(R^{\prime}\right)$ in the argument of the second exponential in the above integral is approximately unity. Because the total Doppler phase departs very little from the mean phase, the argument of the first exponential is small. As a consequence, we expect of the integral to yield approximately the same distribution as in the case for no mean flow (Raleigh distributed amplitude and random phase). This expectation has been confirmed by numerical evaluations of the integral. It was observed numerically that the random component of the phase (phase wander) was very similar to the phase obtained from the "random walk" discussed earlier. In all cases the random phase produced by the integral was much less than the mean phase $\bar{\phi}(R)$.

Fig. 2.5 shows the simulated echo return for scattering from turbulence. On any given realization, the signal will be different, due to the random nature of the scattering which is simulated with a random number generator. The unwrapped phase associated with the simulated echo return is shown in Fig. 2.6: the blue line is the unwrapped phase and the red line is the mean unwrapped phase (no turbulence effects). The difference in the two lines is a measure of the Doppler phase wander. The mean unwrapped phase was computed using the mean (deterministic) wind speed profile that served as an input to the calculation. Note that the random phase produced by scattering (phase wander) from turbulence does not significantly corrupt the mean phase. The simulations thus provide strong theoretical evidence for the stability of the Doppler phase.

\subsubsection{Using Doppler Phase to Extract Wind Speed Profiles}

The numerical observation that the phase of the SODAR signal is dominated by the mean phase $\bar{\phi}(R)$ motivated an examination of the measured "unwrapped" phase of the signals obtained from the ASC SODAR. Fig. 2.7 and 2.8 show, respectively, three consecutive returns on the same beam and the corresponding unwrapped phase, both recorded with the ASC SODAR. The data are plotted as a function of height instead of time. The data were collected as part of the SODAR testing at the University of Mississippi Biological Field Station during the winter of 2010-2011. The data shown are for the v-beam (y-axis) of the ASC SODAR and were taken near midnight on the night of January 7, 2011. The three pulses shown are each separated by 13.6 seconds, which is the time for one complete cycle on the ASC SODAR. Note that in Fig. 2.7, the pulse amplitudes bear little detailed resemblance to each other, giving the impression that there is no systematic information in the signals. In contrast, when the unwrapped phase versus height is plotted (2.8), we see that the three phases are very similar. Note further that the random "wiggles" in the phase are relatively small compared 


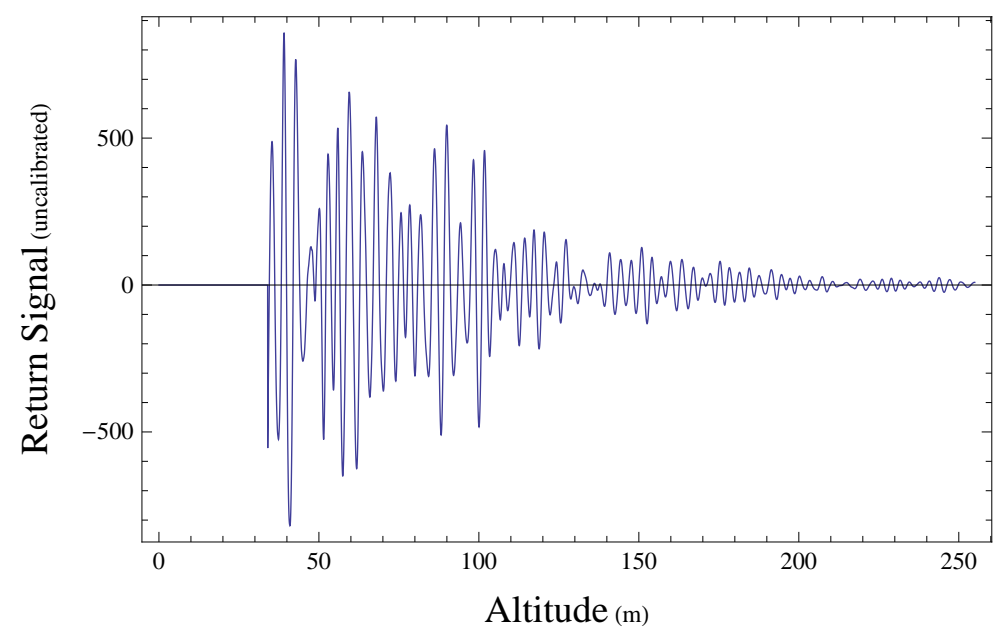

Fig. 2.5. Simulated pressure-vs-altitude signal for the echo received on a SODAR beam (note: Altitude $\left.=c_{0} t / 2\right)$.

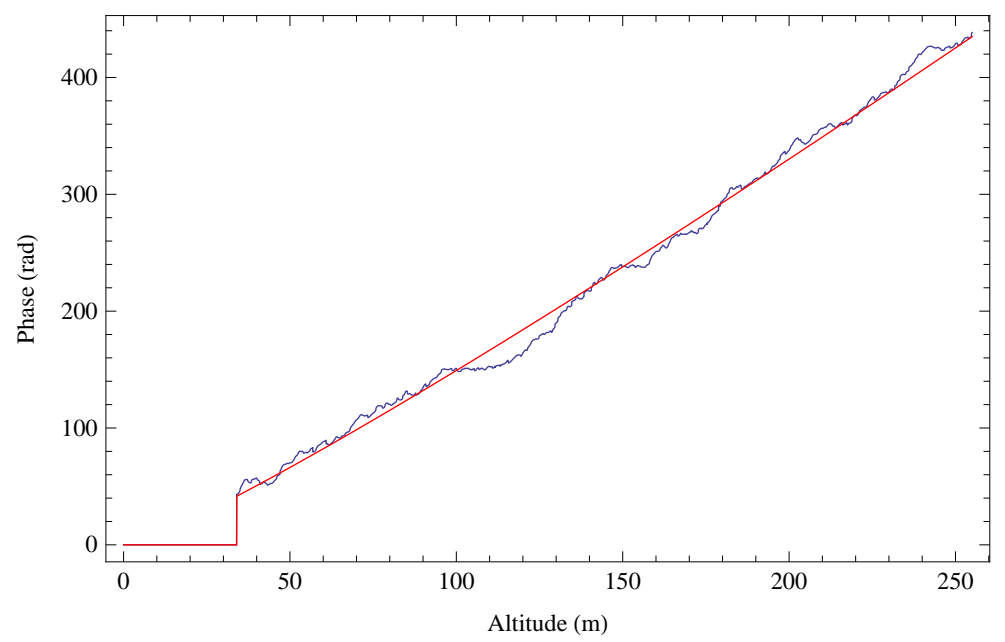

Fig. 2.6. The unwrapped Doppler phase versus altitude for the signal in Fig. 2.5. 


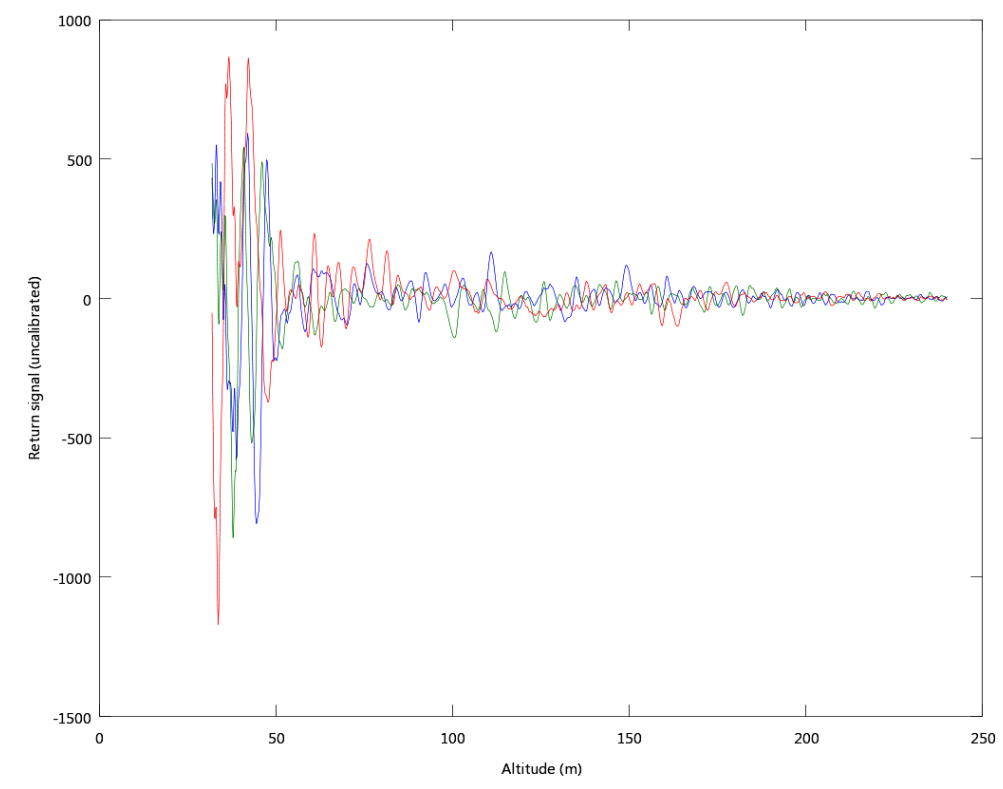

Fig. 2.7. Pressure-vs-altitude for three consecutive $v$-beam echoes separated by 13.6 seconds. The signals were recorded on the ASC SODAR at the University of Mississippi Biological Field Station near midnight on January 7, 2011. Note the similarity of the measured signals to the simulated signal in Fig. 2.5.

to the overall trend in the phase, as was also observed in the numerical simulations. This is experimental evidence that the phase wander does not significantly corrupt the mean phase.

There clearly is order hidden in the apparently random pulses. The conclusion is that the amplitude of the SODAR return reflects the randomness of acoustic scattering from turbulence while the phase shows the systematic behavior of the mean wind speed as a function of height. These suggest that the phases of the instantaneous SODAR returns contain information that can be used to estimate real-time or near-real-time profiles for the wind speed versus height. The ability to obtain systematic information to estimate the wind speed versus height without long-term temporal averaging indicates the potential to utilize SODARs for continuous monitoring of the wind speed in applications for the wind turbine industry. The investigation thus moved forward toward a field experiment conducted adjacent to an instrumented 200-m meteorological tower at Texas Tech University in June of 2011 .

\subsection{Measurements at the WiSE Meteorological Tower}

On June 21-23 of 2011, the ASC SODAR made continuous measurements adjacent to a 200-m meteorological tower at the Texas Tech University (TTU) Wind Science and Engineering Research Center (WiSE). The tower's sonic anemometers were used for comparison to the SODAR data. The SODAR was approximately $150 \mathrm{~m}$ to the west and slightly south of the tower. No attempt was made to correct the time series from the SODAR and tower 


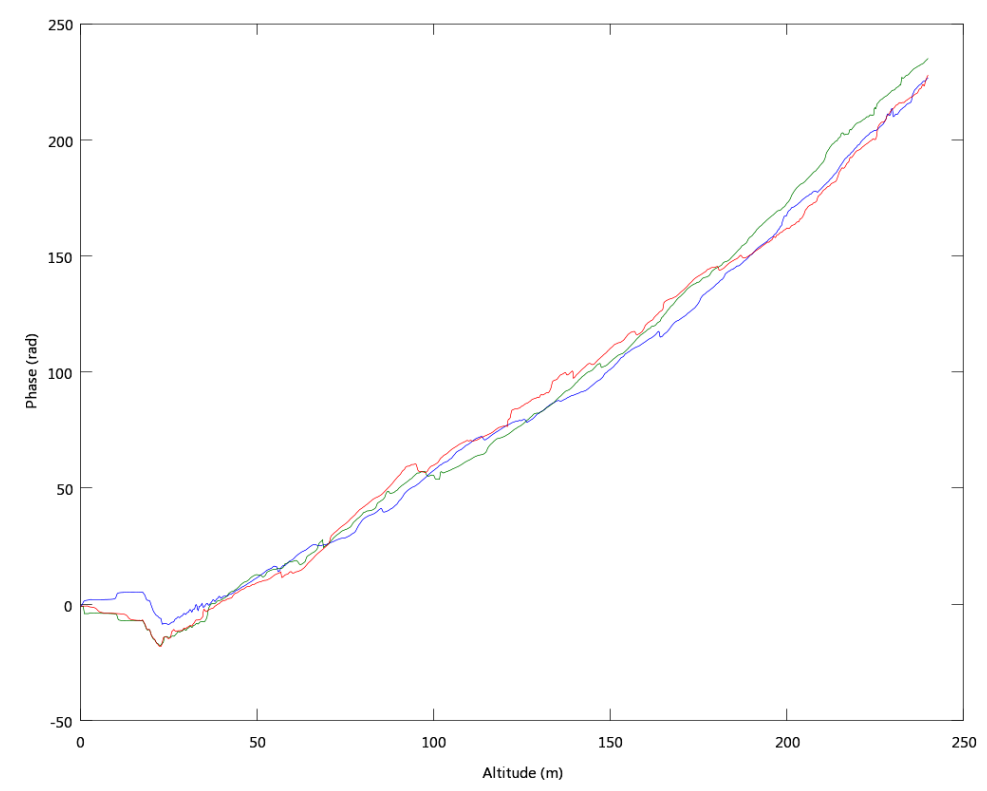

Fig. 2.8. The unwrapped Doppler phases for the echoes shown in Fig. 2.7. Note the similarity of the measured phases to the simulated unwrapped Doppler phase in Fig. 2.6.

anemometers for the time delay associated with convection over the separation distance. Complete details of the TTU field deployment are given in Section 3.5.2.2.

The sonic anemometers on the tower were sampled at $20 \mathrm{~Hz}$ but were down sampled to 1 $\mathrm{Hz}$ for comparison with the SODAR. The ASC SODAR requires approximately 4.5 seconds for the completion of each pulse. Since there are three beams $(u, v$, and $w)$, a complete SODAR cycle takes 13.5 seconds. Thus, a new SODAR wind speed profile is produced every 13.5 seconds.

\subsubsection{Standard Fourier Analysis of TTU SODAR Data}

The first goal was to compare the tower data to the 15-minute averaged profiles produced by the ASC SODAR using the standard ASC SODAR processing which is based on fast Fourier transforms (FFTs). This step was essential in order to verify that the standard ASC wind speed outputs are sufficiently accurate for a useful analysis. These outputs are the standard outputs produced by the ASC software as part of their commercial package.

In the ASC FFT analysis, the SODAR signal on each beam is divided into "time bins" that correspond to altitude bins of $10 \mathrm{~m}$ vertical extent. The $2800 \mathrm{~Hz}$ SODAR carrier wave is removed from the signal and an FFT is computed on the signal in each time bin for each beam. The peak in the FFT gives an estimate of the Doppler shift in each time bin. As discussed earlier, the Doppler shift on each beam is directly proportional to the component of the vector wind velocity in the beam direction.

Due to the finite vertical extent of the sampling volume (34 m for a $0.2 \mathrm{~s}$ pulse), the first usable altitude bin starts at approximately $50 \mathrm{~m}$. At higher altitudes the received echoes die away rapidly, so that the signal-to-noise ratio (SNR) decreases rapidly with altitude. It was 

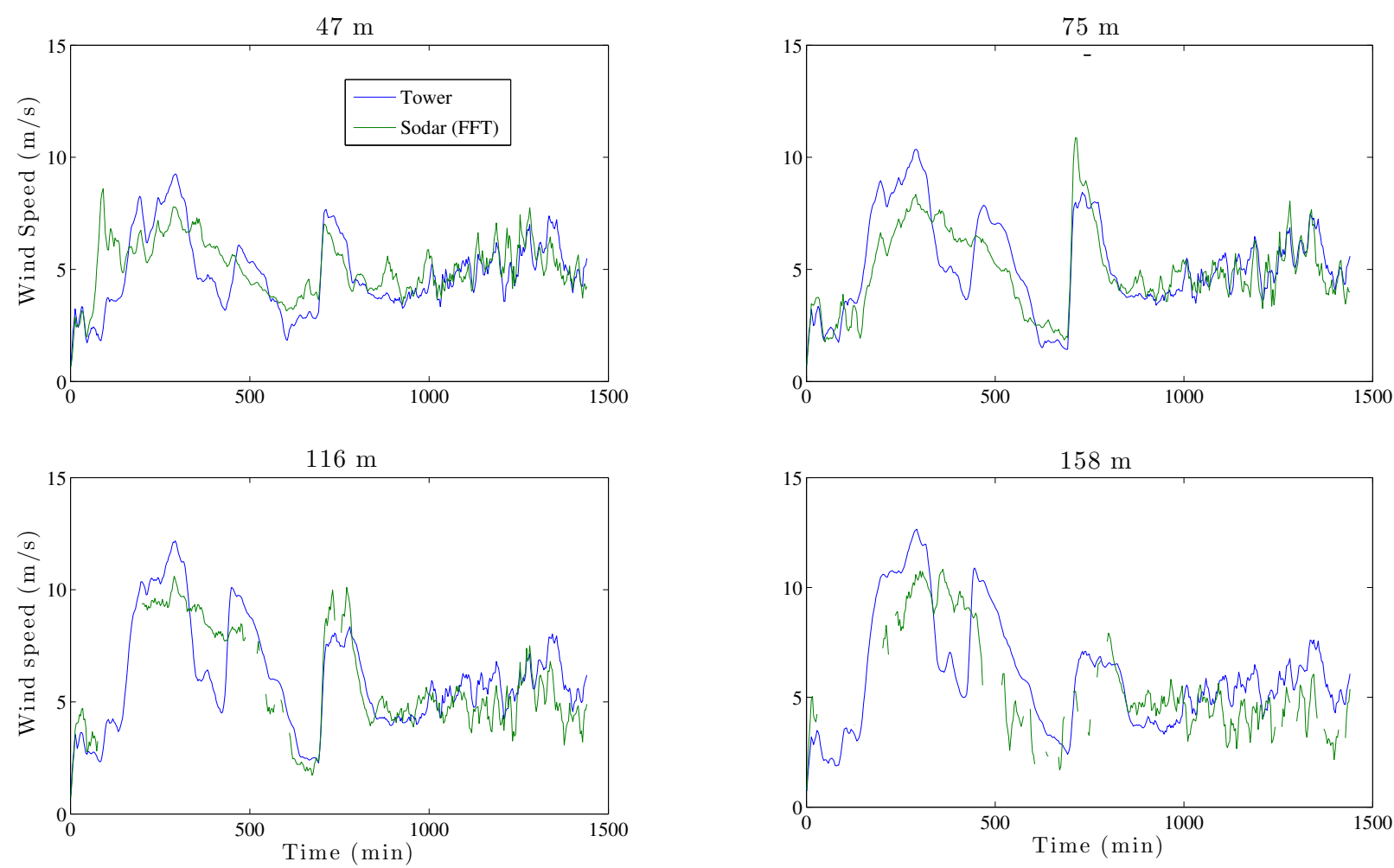

Fig. 2.9. Comparison of wind speeds measured by tower anemometers and estimated by conventional FFT analysis using the ASC SODAR software (15-minute averages).

found that the highest usable altitude was between $150 \mathrm{~m}$ and $175 \mathrm{~m}$. Within these upper and lower limits, there were 4 sonic anemometer locations on the meteorological tower: 47 $\mathrm{m}, 75 \mathrm{~m}, 116 \mathrm{~m}$, and $158 \mathrm{~m}$. The sonic anemometer data from these 4 heights are the tower measurements with which the ASC SODAR was compared.

Fig. 2.9 shows a comparison of the standard ASC SODAR wind speed estimation with the tower sonic anemometers at the heights cited above. Here, only the 24 hour period from $7 \mathrm{pm}$ (00:00 UTC) June 21 to $7 \mathrm{pm}$ June 22 is considered. The blue line is the tower data computed with a 15-minute running average. The green line is the corresponding 15-minute average wind speed from the ASC SODAR. Sunrise in Lubbock, Texas at 6:38 am on June 22 , is 698 minutes into the measurement period.

The ASC SODAR seems to capture the trends, but fails to consistently track the larger excursions particularly during stable atmospheric conditions. In the second half of the 24 hour period, daytime unstable conditions, the tower/SODAR agreement is somewhat better.

The increased turbulence during daytime conditions definitely served to increase the signal-to-noise ratio (SNR). The poor SNR in the first half of the measurement period may be responsible for the marginal agreement with the tower data during that time. Evidence for the poor SNR can be seen in the data dropouts in standard ASC SODAR outputs at the two highest tower heights $(116 \mathrm{~m}$ and $158 \mathrm{~m})$ in the first half of the measurement.

At approximately the middle of the June 22 measurement period, there was a wind speed increase from $2 \mathrm{~m} / \mathrm{s}$ to nearly $10 \mathrm{~m} / \mathrm{s}$ that occurred in less than 2 minutes. This sudden 
increase in wind speed and the subsequent wind speed data are later examined in detail as a test for the usefulness of SODARs for real-time monitoring of rapidly changing wind conditions.

\subsubsection{Doppler Phase Analysis of the SODAR Data}

Doppler phase analysis (using unwrapped phase) has some distinct advantages over the standard FFT analysis. Although the two are mathematically equivalent, Doppler phase analysis lends itself more readily to theoretical analysis than standard FFT analysis. First, Doppler phase analysis does not require peak finding as in the FFT approach. Second, the phase is a continuous function of height, so that continuity constraints are easier to impose. With the FFT approach, in each height bin, each wind speed is essentially an independent estimate, making constraints awkward to apply. Finally, with the Doppler phase approach, once the phase is unwrapped, it is straightforward and efficient to compute the Doppler shifts needed for a vertically continuous wind speed profile. The instantaneous Doppler shift is simply the derivative (time or space) of the phase. That is, $\Delta f=d \phi / d t=\left(c_{0} / 2\right) d \phi / d R \approx$ $\left(c_{0} / 2\right) \Delta \phi / \Delta R$.

Here the mathematical equivalence of using a windowed FFT versus a phase derivative to determine the Doppler shift in a SODAR signal is demonstrated first. Then, the two approaches are applied to the SODAR data and compared.

\subsubsection{Equivalence of Conventional FFT Analysis and Doppler Phase Analysis}

To begin we first treat a Gaussian window function so that a closed form result is obtained. Consider the analytic signal from a SODAR with the carrier wave removed. We assume that the amplitude is normalized to unity, so that the analytic signal is of the form $S_{A}(t)=$ $\exp [i \phi(t)]$, where $\phi(t)$ is the phase. A standard approach for obtaining the Doppler shift, and hence the wind speed, is to compute a windowed FFT on $S_{A}(t)$ and then find the frequency for the peak in the resulting spectrum. For a Gaussian window centered on $\bar{t}$, $\exp (t-\bar{t})^{2}$, the Fourier transform is given by

$$
\tilde{S}(\omega)=\int_{-\infty}^{\infty} e^{-i \omega t} e^{i \phi(t)} e^{-(t-\bar{t})^{2} / \tau^{2}} d t
$$

where $\omega$ is the angular frequency, $\bar{t}$ is the center of the time interval and $\tau$ is the decay constant of the window. If we expand $\phi(t)$ linearly about $\bar{t}$ we have $\phi(t) \approx \phi(\bar{t})+\phi^{\prime}(\bar{t})(t-\bar{t})$, where $\phi^{\prime}(\bar{t})$ is the derivative $d \phi / d t$ evaluated at $t=\bar{t}$. Defining $t^{\prime}=t-\bar{t}$, and using the linear expansion of $\phi(t)$ yields

$$
\tilde{S}(\omega)=e^{i \phi(\bar{t})} e^{-i \omega \bar{t}} \int e^{i\left(\omega^{*}-\omega\right) t^{\prime}} e^{-t^{\prime 2} / \tau^{2}} d t^{\prime}
$$

where $\omega^{*} \equiv \phi^{\prime}(\bar{t})$. Performing the integration yields

$$
\tilde{S}(\omega)=\tau \sqrt{\pi} e^{i \phi(\bar{t})} e^{-i \omega \bar{t}} e^{-\left(\omega^{*}-\omega\right)^{2} \tau^{2} / 4} .
$$

Clearly the peak in the spectrum $|\tilde{S}(\omega)|^{2}=\tau \sqrt{\pi} e^{-\left(\omega^{*}-\omega\right)^{2} \tau^{2} / 4}$ is at $\omega=\omega^{*}=\phi^{\prime}(\bar{t})$. Hence, rather than doing a windowed Fourier transform and locating the peak in $|\tilde{S}(\omega)|^{2}$, we can just evaluate $\phi^{\prime}(\bar{t})$. 
Continuing, a general result can be simply derived for an arbitrary symmetric window function $W(t-\bar{t})$ centered about $\bar{t}$. We again assume that the phase can be expanded linearly about $\bar{t}$, so that we have

$$
\begin{aligned}
\tilde{S}(\omega) & =\int_{-\infty}^{\infty} e^{-i \omega} e^{i\left[\phi(\bar{t})+\phi^{\prime}(\bar{t})(t-\bar{t})\right]} W(t-\bar{t}) d t \\
& =e^{i \phi(\bar{t})} e^{-i \omega \bar{t}} \int e^{i\left(\omega^{*}-\omega\right) t^{\prime}} W\left(t^{\prime}\right) d t^{\prime},
\end{aligned}
$$

where $t^{\prime}=t-\bar{t}, \phi^{\prime}=d \phi / d t=\omega^{*}$. Hence the spectrum is given by

$$
|\tilde{S}(\omega)|^{2}=\left|\tilde{W}\left(\omega-\omega^{*}\right)\right|^{2},
$$

where $\tilde{W}(\omega)$ is the Fourier transform of $W(t)$. For an arbitrary smooth symmetric window, the stationary phase point is at $\omega=\omega^{*}$, and $|\tilde{S}(\omega)|^{2}$ will thus be a maximum at $\omega^{*}=\phi^{\prime}(\bar{t})$. Hence, just as with the Gaussian window function, instead of Fourier transforming $S(t)$ over a window and finding the peak, we can obtain the same result by computing the derivative of the phase, $\phi^{\prime}(\bar{t})$, at the center of the window. The result applies to any smooth symmetric window function.

The only drawback with Doppler phase analysis appears to be the difficulty of accurately unwrapping the phase of noisy data. For the analysis here, the standard MATLAB phase unwrapping routine was adequate for the data considered. To provide a complete analysis, it remains to quantify the uncertainty due to peak finding in the FFT method versus phase unwrapping in the phase analysis method.

\subsubsection{Comparison of Conventional FFT SODAR Analysis and Doppler Phase Analysis}

Here the Doppler shift is estimated by computing the spatial phase derivative using a finite difference approximation. As with the standard FFT approach above, a 15-minute running average is presented. The purpose of the comparison, shown in Fig. 2.10, is to demonstrate the equivalence of the FFT and Doppler phase approaches. The blue line is the same tower data as in Fig. 2.9. The green line was computed by averaging the phase over a vertical interval of $20 \mathrm{~m}$ and approximating the phase derivative with a finite difference also over a $20 \mathrm{~m}$ vertical interval.

Comparison of Fig. 2.9 with Fig. 2.10 shows that the two methods give similar results. It seems that the standard FFT method follows the tower data more accurately. However, with the Doppler phase approach, it was possible to compute wind speed estimates at heights (such as $158 \mathrm{~m}$ ) where, with the FFT approach, there were numerous data dropouts (due to poor SNR). The reason there are no data dropouts with the Doppler phase method is that the phase is continuous. If a poor SNR region has good SNR regions on either side, the Doppler phase method essentially interpolates between the two good SNR regions. Hence if the regions of low SNR are not too numerous, the Doppler phase method will work at all heights under conditions where the FFT method will fail. However, if the SNR becomes sufficiently low, obviously both methods will fail.

It is anticipated that the phase approach could be improved by addressing the phase unwrapping method and the finite differencing used to compute the derivative. 

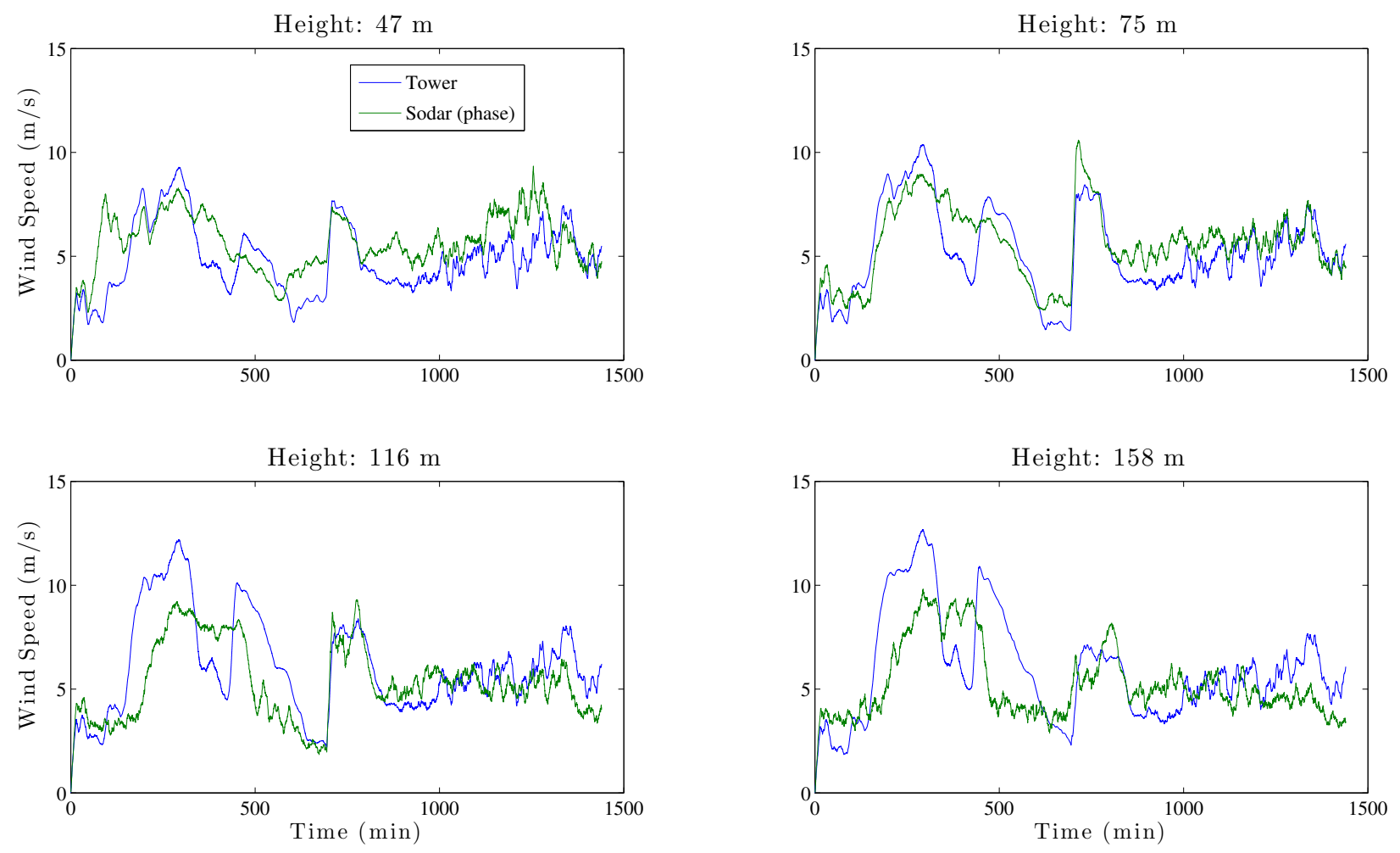

Fig. 2.10. Same as in Fig. 2.9 except the ASC SODAR data was analyzed using a Doppler phase method. 


\subsubsection{Tower/SODAR Comparison: Near Real-Time Data}

A detailed comparison has been made of near real-time tower and SODAR data over three portions of the measurement period: minutes $680-720$, minutes $1010-1050$, and minutes $1300-1340$. These comparisons are shown in Figs. 2.11-2.13. The blue lines are the sonic anemometer wind speeds and the green lines are the wind speeds estimated with the phase derivative method discussed above. The anemometer at $116 \mathrm{~m}$ appears to have an intermittent electronic problem that caused random "spikes" in the wind speed. The spikes did not appear in the 15-minute averages but are apparent in the 1-second wind speed in Fig. 2.11.

The tower sonic anemometer data clearly show the increase in the wind speed fluctuations with a rising sun and increased solar heating. The wind speed fluctuations peak at midafternoon and decrease later in the day as the sun became lower in the sky. In contrast, the fluctuations in the SODAR wind speed estimates stay about the same, as would be expected from the theoretical model presented earlier. That is, the size of the phase fluctuations are relatively insensitive to the turbulence intensity. However, the accuracy of the wind speed estimates are quite sensitive to the SNR. At the highest altitude, $158 \mathrm{~m}$, where the SNR is lowest, the SODAR wind speed estimates are noticeably worse than at the lower altitudes where the SNR is higher.

\subsection{Summary \& Conclusions}

A theoretical model for the instantaneous wave form measured by a Doppler SODAR was presented. The model predicts that, although the amplitude of the received echo is chaotic and apparently random, the unwrapped Doppler phase is quite stable and systematic. Analysis of data confirmed the model predictions and led to a useful approach for analyzing near real-time SODAR data. The approach, which uses the unwrapped phase of the echo, is denoted the "Doppler phase method." The new method has some distinct advantages over conventional FFT processing. The Doppler phase method provides vertically continuous wind speed profiles and can return velocity profiles for individual SODAR echoes allowing for near-real-time output.

The Doppler phase method was applied in a comparison of 24 hours of wind speed data acquired from the WiSE 200-m instrumented tower with wind speeds estimated by an Atmospheric Systems Corporation (ASC) Doppler SODAR. The capability of the ASC SODAR to track rapid changes in wind speed using the Doppler phase method was demonstrated. However, the variances in the estimated wind speeds were larger than desired. This stems from the sensitivity of the Doppler phase method to the signal-to-noise ratio. Traditional FFT methods for SODAR analysis use time-averaging and peak finding to enhance the output. While the Doppler phase method ideally provides a continuous vertical profile for each pulse, applicability of the method would require further research to determine methods for applying physical models to enhance the SNR.

One such method could be to develop an adaptive Kalman filter that should produce smaller variances. This would depend on a realistic parametric model for the spatial-temporal evolution of the second-order statistics of boundary layer turbulence. Secondly, improved models are needed for the spatial correlation and level of the noise in SODAR returns as 

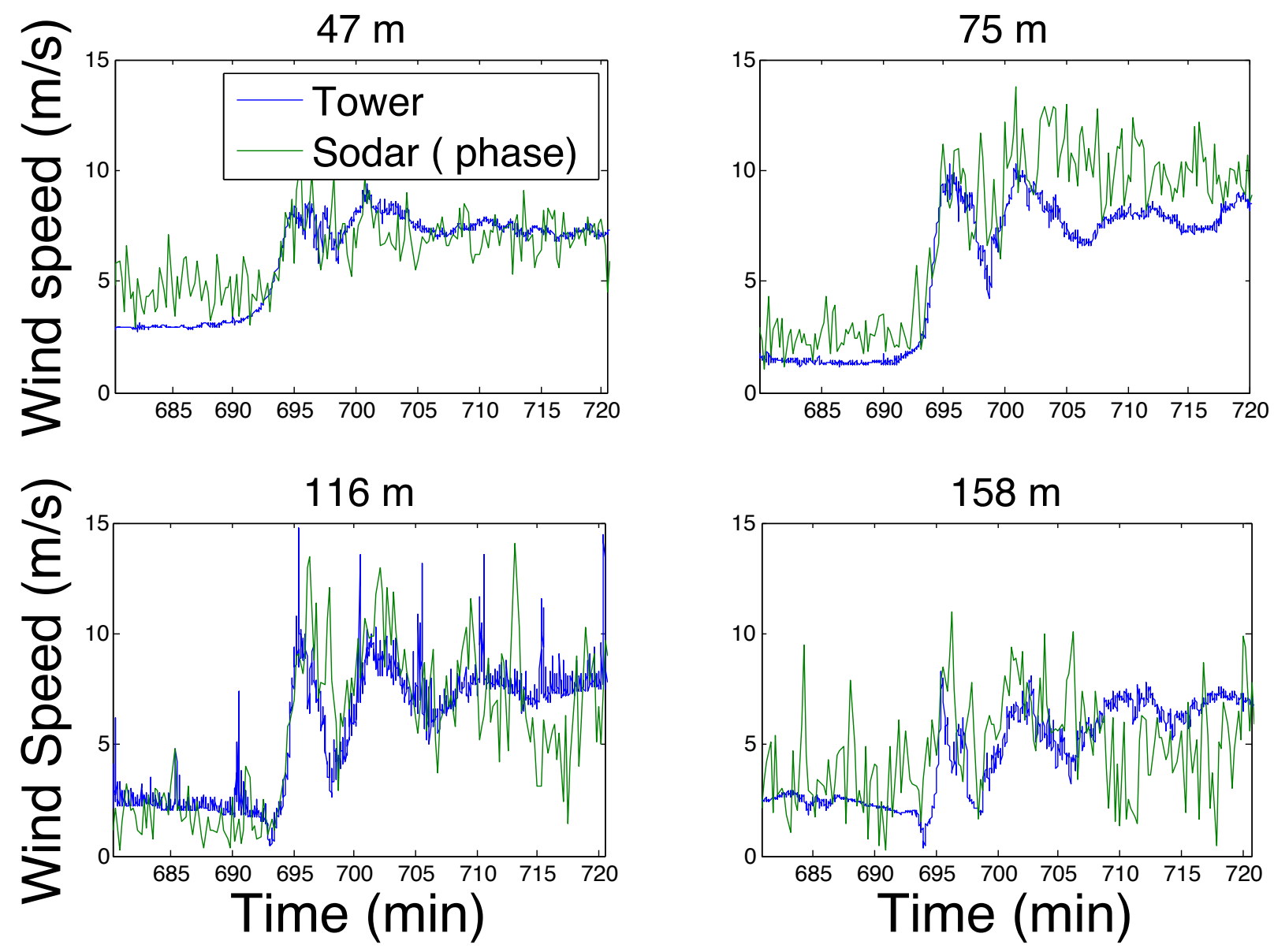

Fig. 2.11. Near real-time comparisons of tower anemometer wind speeds with ASC SODAR estimates using the Doppler phase method for minutes 680 - 720 in Fig. 2.9. The anemometer data rate is 1 sample per second while the SODAR data rate is 1 sample per 13.6 seconds. 

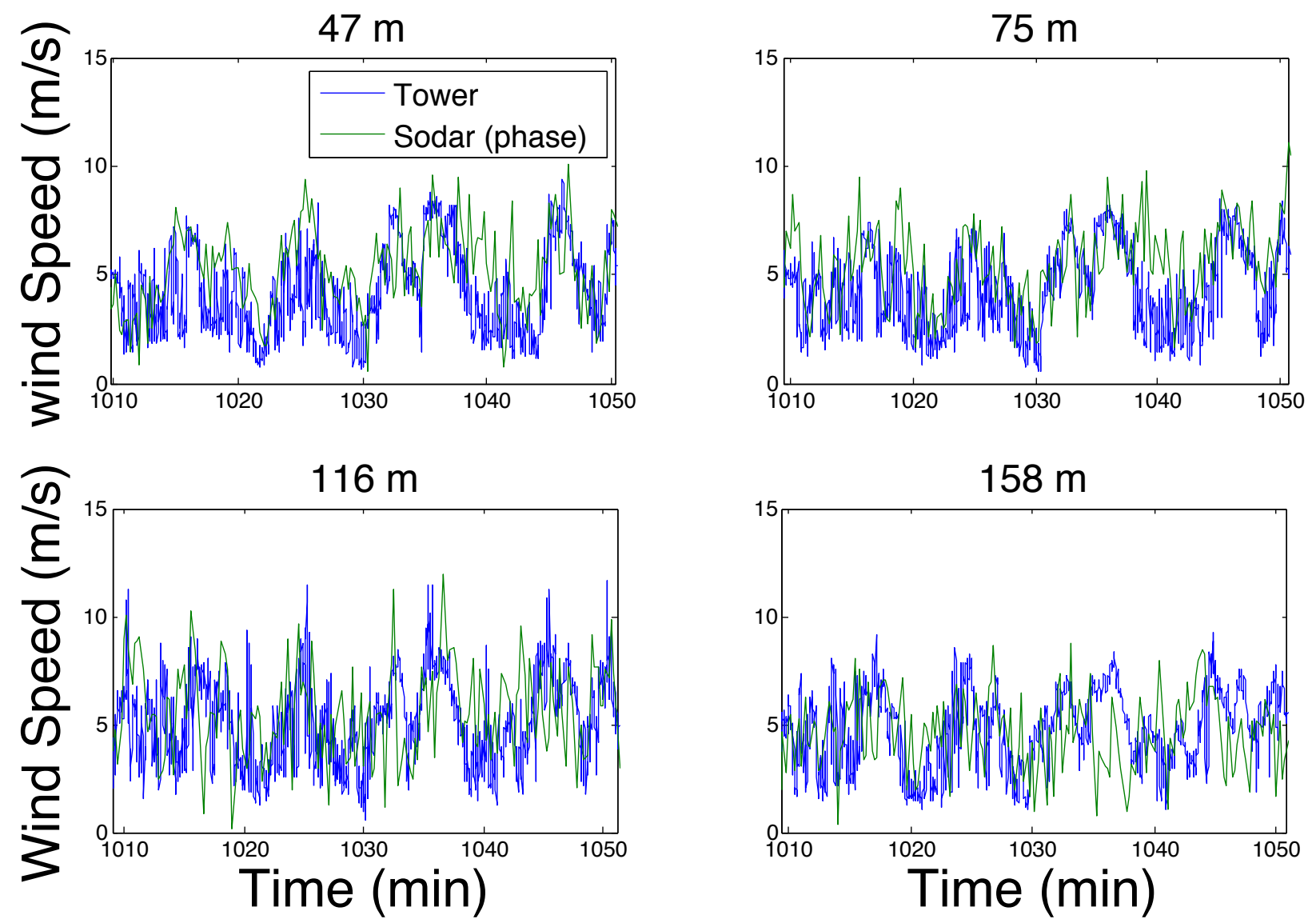

Fig. 2.12. Same as Fig. 2.11 except the time is $1010-1050$ minutes. 

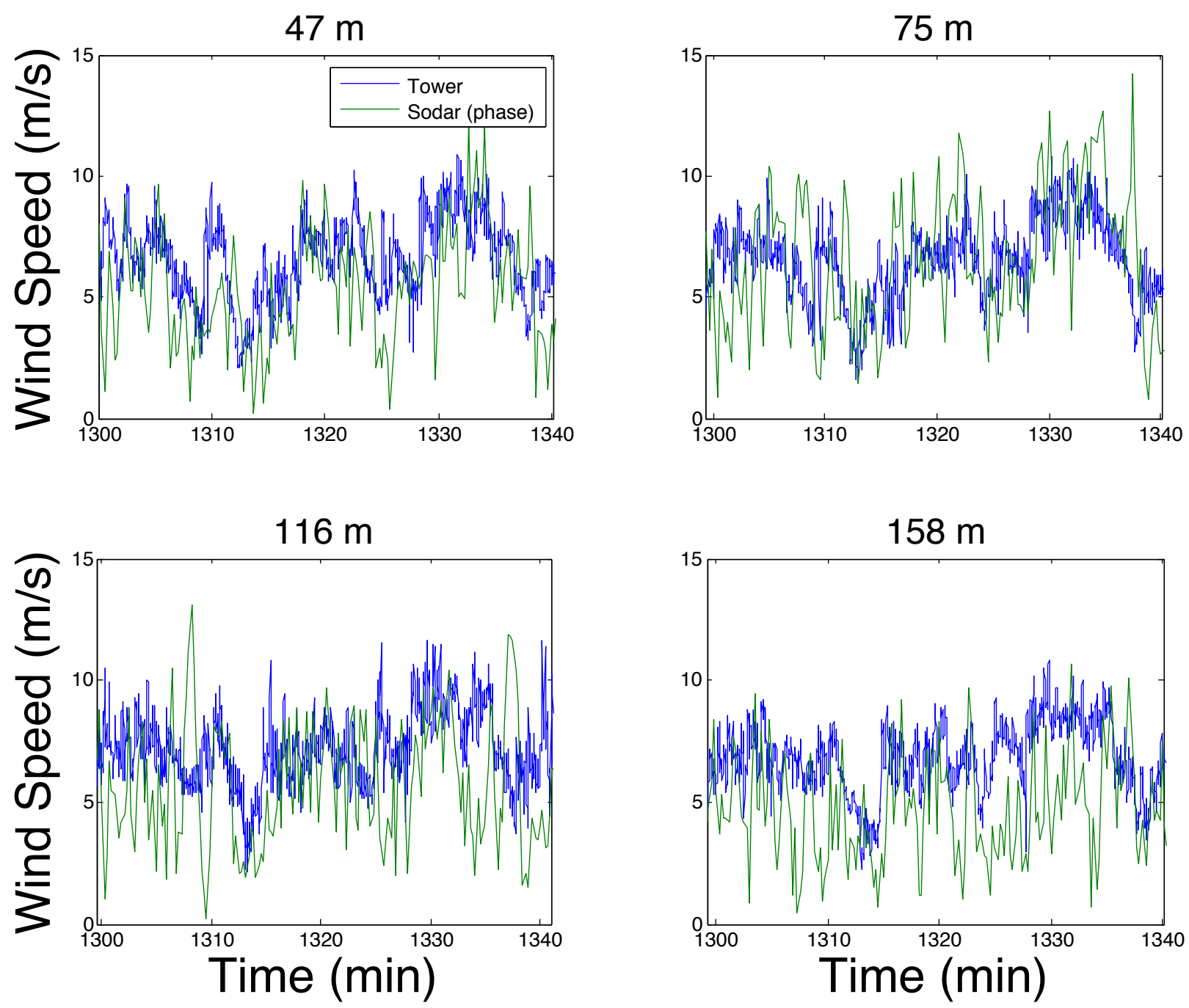

Fig. 2.13. Same as Fig. 2.11 except the time is $1300-1340$ minutes. 
a function of height. Clearly, noise in echo return data will not be uncorrelated sampleto-sample, and this fact should be accounted for in order to not place undue weight on noisy measurements. Thirdly, a more sophisticated method for handling errors in phase unwrapping is needed.

\section{Bibliography}

[1] Antoniou, I.; Jørgensen, H.; Ormel, F.; Bradley, S.; von Hünerbein, S.; Emeis, S.; \& Warmbier, G. (2003) "On the Theory of Sodar Measurement Techniques." Ris $\varnothing$ National Laboratory, Final Report on WP1, EU WISE Project NNE5-2001-297 Ris $\varnothing-$ R-1410, Roskilde, Denmark.

${ }^{[2]}$ Brown, R. G. \& Hwang, P. Y. C. (1997) Introduction to Random Signals and Applied Kalman Filtering. John Wiley \& Sons.

[3] Crescenti, G. (1997) "A Look Back on Two Decades of Doppler Sodar Comparison Studies." Bulletin of the American Meteorological Society, Vol. 78(4):651-673.

${ }^{[4]}$ Maybeck, P. (1979) Stochastic Models, Estimation And Control, Vol. 1. Academic Press, New York.

${ }^{[5]}$ Rodberg, L. \& Thaler, R. (1967) Introduction to the Quantum Theory of Scattering. Academic Press, New York.

[6] Seibert, P. (1998) "Long-time Comparison of Remtech Pa2 Sodar Wind and Turbulence Measurements with Cabauw Tower Data." In Proc. 9th International Symposium on Acoustic Remote Sensing of the Atmosphere and Oceans (ISARS), pp. 136-139, Vienna, Austria. 


\section{3 | Passive Acoustic In-Flow Profiling}

\subsection{Concept Description}

As noted by Kelley et al., unsteady inflow conditions caused by turbulence in the atmospheric boundary layer (ABL) lead to unsteady blade loading which contributes to turbine failure. ${ }^{[8]}$ Additionally, the inability to predict the inflow conditions for seasonal and/or diurnal variation lead to suboptimal performance compared to expectations. ${ }^{[3]}$ The current industry methods for addressing these issues include measurement systems such as

- LIght Detection and Ranging (LIDAR) at $\$ 50 \mathrm{k}$ to $\$ 100 \mathrm{k}$ per unit,

- SOnic Detection and Ranging (SODAR) at $\$ 30 \mathrm{k}$ per unit, and

- instrumented meteorological towers.

These systems are costly and provide measurement along a single vertical line. It would be prohibitively expensive to deploy a large enough number of these systems to provide real-time feedback for optimization of an entire wind farm.

This program offers a low cost concept to the wind resource measurement community by utilizing relatively low cost ( $\$ 1,200$ per unit) infrasound pressure sensors in a wireless array to sense the local wind conditions around a turbine. The concept proposes the distribution of an array of these infra-sound sensors (0.01-100 Hz sensitivity) on the ground in the area around a wind turbine. Then, using correlation-based analysis methods, the vertical profile of the wind velocities is estimated on a real-time basis. Fig. 3.1 illustrates a conceptual layout for such an array to provide inflow velocities. A pair of microphones is positioned in a number of heading directions to estimate the profile in that direction.

The concept builds upon years of experience in infra-sound propagation in the atmosphere and technologies for its measurement. Section 3.2 discusses this experience at the National Center for Physical Acoustics in detail and states an explicit hypothesis based on this experience that guided the Task 2 effort. Section 3.2 also provides other references in the published literature that support the concept. Section 3.3 gives details on the approach taken to test the hypothesis and provides a review of the theoretical basis for the approach. In section 3.4, the results of a re-analysis of some old infrasound data is presented which gives support to the hypothesis. Section 3.5 describes the large experimental campaign completed under this work to provide data to use in testing the governing hypothesis. Finally, sections 3.6 and 3.7 present results of data analysis efforts performed on the collected data. Overall conclusions and comments on future work are provided in section 3.8

\subsection{Background}

\subsubsection{Development of Primary Hypothesis}

The impetus for the title effort of this research program, "Passive Acoustic Detection of Wind-Turbine Inflow Conditions," is found in experimental results discovered by researchers at the National Center for Physical Acoustics (NCPA) over years of experience in infra-sound monitoring. "Since 1998, NCPA has been leading the national resurgence of the development 


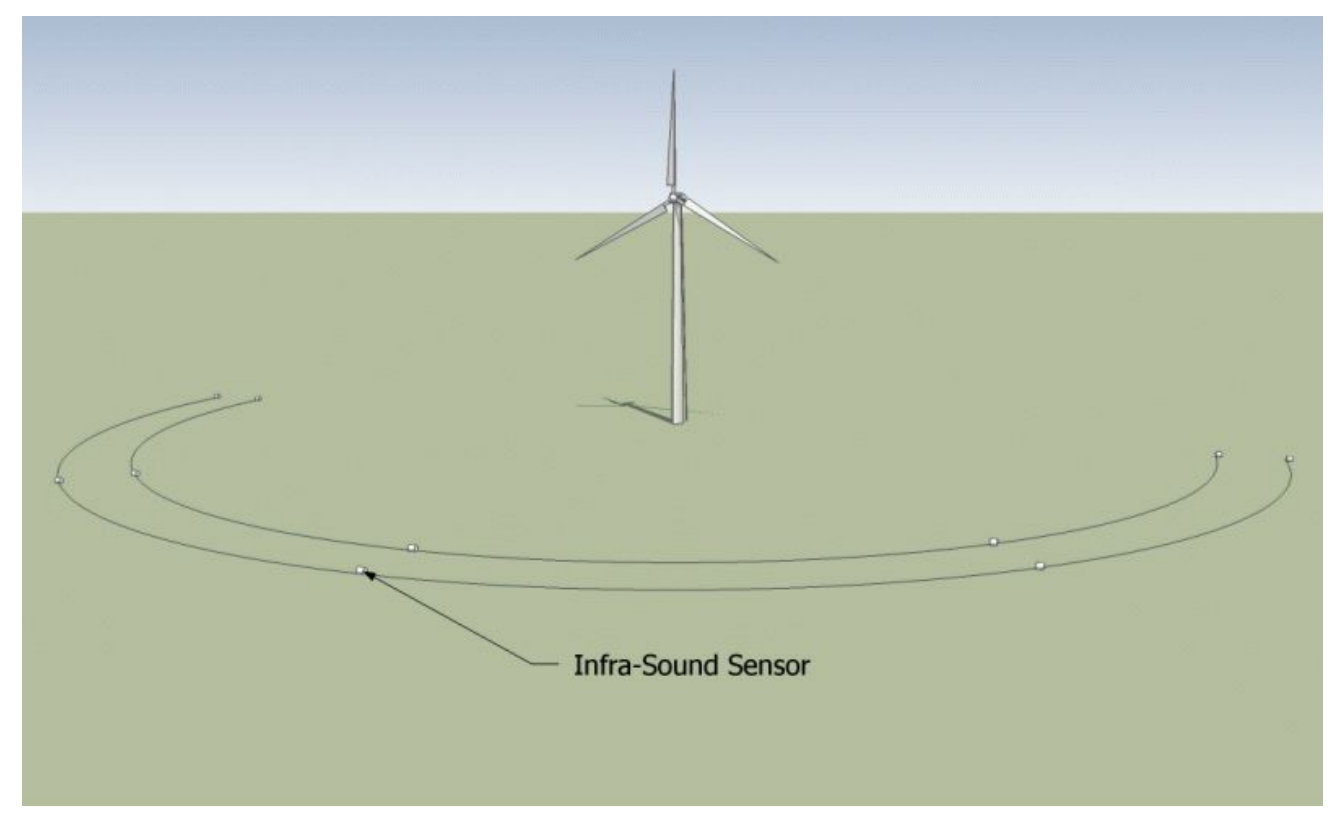

Fig. 3.1. Illustration of the concept for infra-sound detection of wind turbine inflow conditions.

and application of infrasound research and technology. NCPA played a decisive role in the development of the U.S. infrasound component of the United Nations Comprehensive Test Ban Treaty Organizations International Monitoring System (IMS) and has been, and remains, the lead institution for the U.S. Infrasound Research and Development Team. While the IMS was intended primarily for the detection of clandestine nuclear weapons tests, for the last decade the U.S. has been focused on using the infrasound network for geophysical research." *

Typically, the IMS employs infrasound sensing elements that are connected to a network of porous hose. The hose is used to effectively increase the sensing area on the Earth's surface thereby averaging out the effects of atmospheric turbulence. The hose, while effective, ages quickly and needs to be replaced on a regular basis. As an alternative, NCPA researchers began experimenting with large multi-element infra-sound sensor arrays with the intent to average the signals to reduce the effect of wind noise any enhance signal quality. ${ }^{[26]}$

In field testing the multi-element arrays, NCPA researchers began to notice correlated disturbances that neither traveled with the acoustic sound speed nor the seismic speed. These correlated disturbances also appeared to convect at different speeds depending on their correlation length. ${ }^{[4,26]}$ Fig. 3.2 illustrates just such an observation presented in Dillion et al. ${ }^{[4]}$ This was vexing for the physical acoustics researchers but was identified by the aeroacoustics researchers as a familiar trait of turbulent flows. ${ }^{[5]}$ These observations gave rise to the hypothesis that an array of suitable pressure transducers distributed on the ground could be used to predict the time-dependent velocity profile in the near-Earth boundary layer.

\footnotetext{
*http://ncpa.olemiss.edu/infrasound/
} 


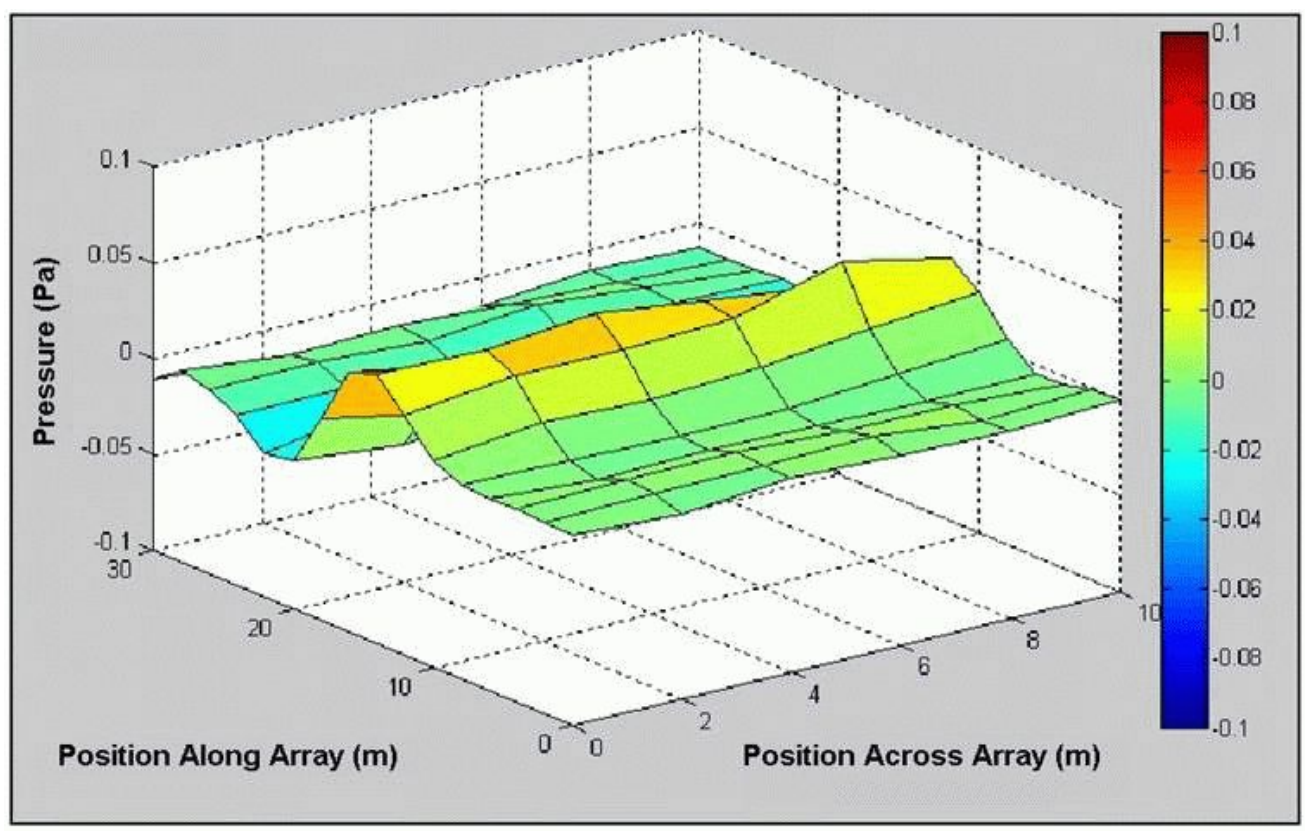

Fig. 3.2. Snapshot of a turbulence-related pressure wave propagating across a 100-element infra-sound sensor array at a speed consistent with the local wind velocity. Taken from Dillion et al. ${ }^{[4]}$

\subsubsection{Infra-Sound Array Observations of Shields ${ }^{[27]}$}

In discussions with Dr. F. D. Shields concerning some data that he had collected with an infra-sound array, it was apparent that his findings were of fundamental importance to the goal of utilizing ground based sensors for estimating wind profiles. These following notes summarize the key points of the discussion.

\subsubsection{Details of Dr. Shields' Data}

The discussion with Dr. Shields centered around an unpublished paper in which the results of data collected at Piñon Flat, CA in September of 2007 were analyzed. The following description of the data is taken from that paper.

"This paper compares signals from a seismometer and a 100 element infra-sound array deployed at Piñon Flat, CA. in Sept. of 2007. The sensors were individually calibrated, had a sensitivity of approximately $2 \mathrm{mv} / \mathrm{Pa}$, and a flat frequency response from 0.02 to $100 \mathrm{~Hz}$. A similar array was previously used in the observation of infra-sound from the second and third high altitude blasts from rockets launched from White Sands Missile Range in 2006. The sensors in the array were arranged in a square, 10 sensors on an edge, with the sensors $2 \mathrm{~m}$ apart, making the array $18 \mathrm{~m}$ square. The signals from the 100 sensors were read and recorded 200 times per second with a 16 bit A/D data acquisition system. Signals from an anemometer located at the center of the array giving the $\mathrm{x}, \mathrm{y}$, and $\mathrm{z}$ components of the wind velocity were also recorded simultaneously. The array was co-located with a calibrated CTBT IMS infrasound pipe array and a seismometer. The original purpose for the deployment was to 


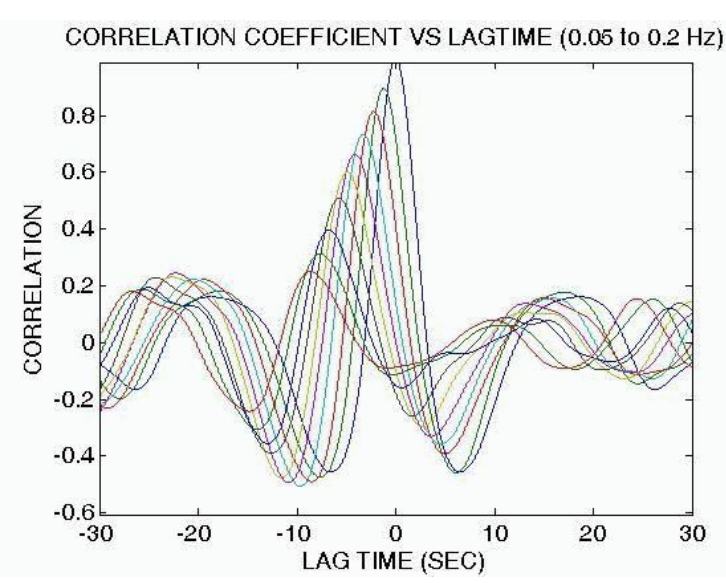

(a) Cross-Correlations

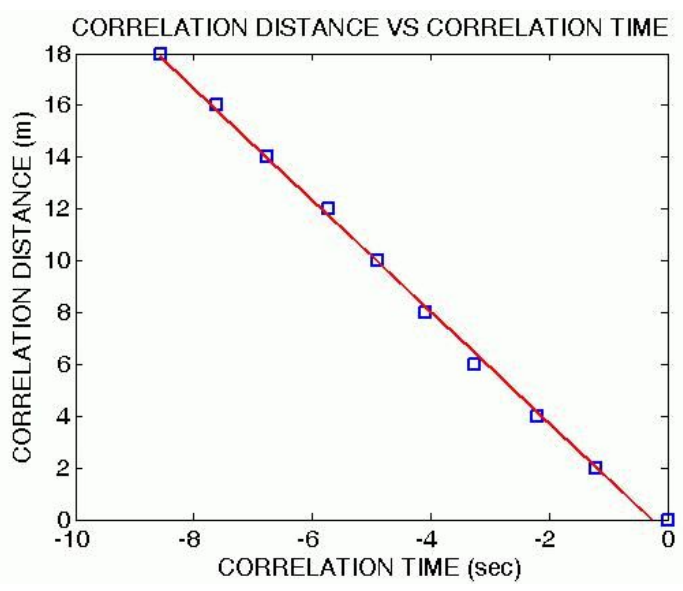

(b) Correlation Peaks

Fig. 3.3. Shields' results from Piñon Flat data showing the cross-correlation of columns in the array running east to west. The slope of the line in $3.3(\mathrm{~b})$ is $-2.15 \mathrm{~m} / \mathrm{s}$.

compare the noise canceling and infra-sound sensing ability of the 100 element array with that of the calibrated pipe array."

The key feature of the data for supporting the present hypothesis is that the infra-sound data were recorded simultaneously with an anemometer which was located at the center of the infra-sound array.

\subsubsection{Shields' Observations Related to Wind Speed Predictions}

Shields' primary observation was the correlation between the infra-sound data and seismic data. However, as a secondary focus, the infra-sound array data was processed to yield crosscorrelations between either rows running south to north or between columns running west to east. The results are particularly telling in relation to the present efforts for wind speed predictions.

\footnotetext{
"When these cross correlations are plotted as a function of lag time, the peaks in the correlation curves are shifted by the time it takes the eddy pattern to move from the first row or column to the row or column in question. When the sensor separation is plotted as a function of this delay time a straight line should result with a slope equal to the wind speed. This effect is shown for a 15 minute interval when the wind was blowing from west to east with a speed of approximately $2.1 \mathrm{~m} / \mathrm{s}$ in Fig. 3.3."
}

Analysis of the slope in Fig. 3.3(b) gives a wind speed of $-2.15 \mathrm{~m} / \mathrm{s}$ which matches the wind speed Shields measured with an anemometer located at the center of the infra-sound array at approximately 5 to 6 feet off the ground.

\subsubsection{Brief Review of Relevant Literature}

As turbulence boundary layers are a significant area of research, there is no end to the number of relevant publications discussing characteristics of the same. In this particular 
effort, the researchers found a selection of these of direct significance. These included publications related specifically to the wall-pressure fluctuations. ${ }^{[2,6,10,12-14,16,17]}$ In addition to these, there are three publications that were key significant references in support of the working hypothesis. These are reviewed here in brief.

\subsubsection{Wind Speed from a Three Microphone Array, Bass et al. ${ }^{[1]}$}

This early work by NCPA researchers found that Taylor's frozen turbulence hypothesis could be applied to determine the local wind speed and direction from a three microphone array. The approach was simply "to determine the wind speed acoustically" using "the travel time between microphones" for the signal associated with turbulent eddies. "The travel time is determined from a cross-correlation between pairs of microphones at known locations."

The researchers note that "the observed noise is most intense below $50 \mathrm{~Hz}$." In conclusion, they state that "initial results indicate that average wind speed and direction can be determined from the wind noise on a microphone array."

This early work only focused on the local wind velocity near the measurement microphones. In contrast, the present work seeks to utilize the frequency dependent phase lag to determine the wind speed at other altitudes above the measurement array.

\subsubsection{Correlation Studies of Priestly ${ }^{[18,19]}$}

J. T. Priestley's work titled, "Correlation Studies of Pressure Fluctuations on the Ground Beneath a Turbulent Boundary Layer," which he completed in 1965 at the University of Maryland while working with Dr. Alan Faller, is a key reference for the current effort. This is because Priestly successfully established an empirical model for the frequency dependent cross-correlation delay and predicted the atmospheric boundary layer velocity profile.

In his thesis, Priestley defines a coherence scale, $\alpha$, such that the optimal (time shifted to provide maximum) cross-correlation decays with distance such that

$$
R_{o p t, \omega}(\xi)=e^{-\alpha \xi}
$$

Priestley recognizes that the coherence scale should be proportional to the wave-length, $k$, which is related to frequency, $\omega$, by the convection velocity, $k=\omega / U_{c}$. Then, he plots all of the values of $\alpha$ and $k$ for all the data he collected over a significant range of wind speeds and solar radiation magnitudes. He finds that his data above $k=0.02 \mathrm{~m}^{-1}$ can be represented by

$$
\alpha=0.41 k^{1.28}
$$

where both $\alpha$ and $k$ are expressed in $\mathrm{m}^{-1}$. The data for $k<0.02$ seem to suggest a constant value for $\alpha$. Next, Priestley makes the following observation:

Bull, et al. $(1963)^{\dagger}$ find that it is necessary to distinguish between small and large eddies; and, in particular, that for wave numbers less than $k=0.35 / \delta^{\star}$ (the large eddies), the coherence scale is independent of $k$. If we assume that the turbulence

\footnotetext{
${ }^{\dagger}$ Bull, M. K., Wilby, J. F., Blackman, D. R. (1963) "Wall Pressure Fluctuations in Boundary Layer Flow and Response of Simple Structures to Random Pressure Fields." University of Southampton A.A.S.U. Report No. 243.
} 
in the present experiment is similar to that investigated by Bull, et al., and that the behavior of the left hand portion of Fig. 7 [Priestley's plot of $\alpha$ and $k$ ] is the result of the same phenomenon they observed, we can get a rough estimate for the present boundary layer displacement thickness.

Priestley went on to estimate $\delta^{\star}=18 \mathrm{~m}$ and $\delta \approx 200 \mathrm{~m}$ which seems reasonable for an atmospheric boundary layer.

Priestley continues his analysis with a discussion of the convection of eddies of various sizes and makes some very pertinent conclusions about the effect that large eddies will have on the surface pressure correlation at high frequencies due to small eddies:

Now, if we look at the effect of the small eddies upon the coherence of the large eddy pressure fluctuations, we find that the velocity variations due to the small eddies cannot physically displace the large eddies and, therefore, cannot cause any phase variation in the large eddies. The only thing the small eddies can do is to cause local high frequency pressure fluctuations which simply add to the low frequency pressure undulations due to the large eddies. Since we are measuring the narrow band correlations, the high frequency pressure fluctuations added to the low frequency variations cannot affect the coherence in the low frequency pass band.

Finally, Priestley utilizes his observations to obtain a wind velocity profile through the application of the following discussion:

... it is probably safe to assume that the dominant influence of an eddy extends a distance approximately proportional to the "size" of that particular eddy. Thus we would expect the high frequency portion of the fluctuating pressure, the part arising from small eddies, to be caused predominantly by turbulence near the ground. Likewise, the effective source height of the low frequencies should be expected to be higher since the larger eddies extend to a greater height. If this is true, the "size" of the eddy versus the convection velocity should be monotonically related to the height versus wind speed. That is to say, if we let convection velocity correspond to wind speed, then the wavelength should be monotonically related to source height.

Priestley then confirms this conclusion be plotting the correlation length, $\lambda$, as a function of the convection velocity, $U_{c}$. He then confirms this is related to altitude by comparison to two anemometer measurements at 4.27 and 10 meter heights above the ground which exactly intersect the curve! The resulting plot of $\lambda$ vs. $U_{c}$ resembles a boundary layer profile.

Priestley's results for $\lambda$ vs. $U_{c}$ were produced for a solar radiation of $\approx 0.1$ Langley $/ \mathrm{hr}$. Data for $\approx 25$ Langley/hr showed little variation in the convection velocity with respect to wave-length. Priestley concludes that the vertical motion of the air mass in a highly unstable condition results in a nearly uniform convection velocity. This is most likely incorrect and results from the fact that the boundary layer thickness for unstable conditions was too thick to be measured using the array size that Priestley had employed. The arrays used by Priestley had no more than 4 sensors and a maximum linear separation of 45 meters. Therefore, he was only able to predict convection velocities up to heights of $\approx 60$ meters (based on Fig. 9 in his thesis). 


\subsubsection{ABL Research by Klewicki et al. ${ }^{[9]}$}

Klewicki et al. present results of an experimental work performed at the Surface Layer Turbulence and Environmental Science Test (SLTEST) facility on the salt playa in Utah is presented. Measurements were acquired during near-neutral conditions in the evening. Streamwise pressure gradients were measured using 3 microphone types: (a) 0.25 inch LarsonDavis resolving down to $23 \mathrm{~Hz}$, (b) 0.5 inch Larson-Davis resolving down to $1.0 \mathrm{~Hz}$, and (c) 0.5 inch B\&K 4193-L-004 resolving down to $0.07 \mathrm{~Hz}$. In discussing the resolution of these sensors the authors state, "Based on estimates of the depth of the atmospheric surface layer, we may surmise that the $0.07 \mathrm{~Hz}$ transducers capture the contributions from $\delta$ scale eddies. Specifically, typical values for the suface-layer thickness, $\delta$, and the mean velocity at $y=\delta$ are $100 \mathrm{~m}$ and $10 \mathrm{~m} / \mathrm{s}$, respectively. Given these values, a $\delta$-sized eddy advecting at $U_{\infty}$ would generate a frequency of about $0.1 \mathrm{~Hz}$. Comparisons of the present data with the model predictions of Panton \& Linebarger (1974) further support the conclusion that the present measurements capture a range of frequencies extending from the high-end to below the peak [pp. 200-201]."

With regard to the wall pressure gradients in the streamwise direction, the authors state, "Axially aligning the array allowed the construction of space-time correlations, $R_{p p}(\Delta x, \Delta t)$. [The advection velocity] $U_{a}^{+}$was calculated by determining the first peak in the correlation between pairs of transducers separated by a distance $\Delta x^{+}$and the simple relation $U_{a}^{+}=$ $\Delta x^{+} / \Delta t^{+}$." Concerning the results the authors state that the calculated values "reveal an increasing trend in $U_{a}$ with increasing $\Delta x^{+}$that is consistent with numerous previous studies (e.g. Willmarth \& Wooldridge 1962; Bull 1967; Blake 1970) [p. 203]." This is extremely significant because it (a) supports the ability to measure convection velocities from surface pressure measurements and (b) provides references showing that this trend is well known from previous work.

The catch will be the determination of the actual velocity, $U^{+}\left(y^{+}\right)$from the advection velocity, $U_{a}^{+}$. The authors comment that the largest $U_{a}^{+}$measured was 18.8 which was significantly larger than previous studies at lower Reynolds number. However, the spacing between sensors was small, so this value is assumed to be a lower bound. This suggests that the relationship between the two quantities may be Reynolds number dependent. This would suggest that wind tunnel measurements at low Reynolds numbers relative to the ABL may not be useful.

The high Reynolds number data was used to update the empirical constants in a relationship between the pressure variance and the boundary layer thickness [p. 207]. This relationship was originally proposed by Farabee \& Casarella (1991) and was update by the authors to

$$
\frac{p_{r m s}}{\tau_{w}}=\sqrt{6.5+2.30 \ln \left(\frac{\delta^{+}}{333}\right)} .
$$

This relationship suggests that it is reasonable to predict $\delta^{+}$using the pressure variance. This may be the key to determining the relationship between the advection velocity, $U_{a}^{+}$, and the actual velocity at a given altitude.

Concerning gusting events the authors reference Schewe (1983) who "revealed that high-frequency high-amplitude negative events in the $p(t)$ signal underlie the negative skew- 
ness" in the p.d.f. of the fluctuating wall pressure [p. 207]. They also note that at $\delta^{+} \simeq 600$ Schewe found that "sensors having a $d^{+}$[normalized diameter $d u_{\tau} / \nu$ ] greater than 50 failed to produce a negative $S(p)$ [skewness]." This gives information concerning the detection of gusting events (i.e. high-frequency, high-amplitude negative events in $p(t)$ ) which may be undetectable if the sensors are too large but should, if detected, manifest themselves in the skewness of the pressure probability density function.

Concerning the pressure spectra, the authors reference Panton \& Linebarger (1974) for a method to calculate the expected spectral shape including the $f^{-1}$ behavior observed by the authors. Noting that the calculations of Panton \& Linebarger assume an irrotational and isothermal free stream, the authors make the interesting statement that "the outer region of the atmospheric surface layer is bounded by a turbulent 'free stream,' and, even under near-neutral stability conditions, will contain motions that formed under unstable conditions [p. 210]."

Concerning the pressure correlations, the authors analyze the auto-correlations and state that the "time to the first zero-crossing has association with advecting $O(\delta)$ eddies [p. 211]." This suggests that the auto-correlation itself may contain enough information for estimating the advection velocity. If this could be confirmed with cross-correlation measurements, it would significantly reduce the number of sensors required to estimated turbine inflow conditions.

The authors examine the $(\partial p / \partial x)_{\text {wall }}$ spectra and find a consistent peak at $f^{+}=0.08$ [p. 214]. They conclude that this reveals that "even at this high Reynolds number the motions involved in the surface flux of vorticity are confined to a relatively narrow band of dynamically significant scales. The frequency associated with this peak corresponds to an advected length of $x^{+} \simeq 285$ [pp. 214-215]." They find that this also manifests itself as a distinctive negative peak at $\Delta t^{+} \simeq 15$ in the autocorrelation of $\partial p / \partial x$.

\subsection{Approach}

\subsubsection{Steps Toward a Deployable System}

In order to effectively determine the wind-turbine inflow conditions, the ground-based system must be able to provide (a) an estimation of the velocity profile over the vertical extent of the turbine and (b) an ability to detect and track gust-type events. The work performed in Task 2 was specifically directed to evaluate these two keys to enabling a realtime acoustic profiler.

Success was anticipated based on previous observations of infra-sound array deployments. The works of Priestly ${ }^{[18,19]}$ and Klewicki et al. ${ }^{[9]}$ discussed above provided some guidance to the efforts. First, as a means of testing the hypothesis, a subcontract was provided to Dr. Shields to re-analyze existing infra-sound array measurements taken at Piñon Flat, CA, in September and October of 2007. These results are discussed in Section 3.4. Second, experiments were planned which would provide the data necessary to assess the key issues. This included a wind tunnel experiment conducted to develop a method for velocity profile estimation and to determine the dependence of the method on the surface temperature. Also, two large field experiments were conducted to acquire infra-sound array data. The deployment at the Reese Technology Center in Lubbock, TX, provided synchronized array data 
with a 200-m meteorological tower. These experiments are described in detail in Section 3.5. The use of correlation-based method to estimate the velocity profile from the surface pressure data is presented in Section 3.6. Finally, the use of low-dimensional methods to detect gust-type events in the ABL from the pressure data is presented in Section 3.7.

\subsubsection{Fundamental Basis for Pressure/Velocity Coupling in the ABL}

Poisson's equation provides a description of the convected pressure field, $P_{c}$, that is the hydrodynamic pressure gradients that seem to move with the flow field, as being dependent on the Laplacian of the turbulence velocity product. Here the velocity, $U$, is decomposed by the familiar Reynolds decomposition into a mean, $U_{\infty}$, and fluctuating, $u$, component such that $U=U_{\infty}+u$.

$$
\frac{\partial^{2}}{\partial x_{k} \partial x_{k}} P_{c}=-\rho_{0} \frac{\partial^{2}}{\partial x_{i} \partial x_{j}} u_{i} u_{j}
$$

Integrating (3.4) gives the pressure on a surface under a turbulent flow. ${ }^{[10,15]}$

$$
P(\vec{x}, t)=\frac{\rho_{0}}{2 \pi} \int_{y_{2}>0} \frac{\partial^{2} u_{i}(\vec{y}, t) u_{j}(\vec{y}, t)}{\partial x_{i} \partial x_{j}} \frac{d^{3} \vec{y}}{|\vec{x}-\vec{y}|}
$$

However, at this point the question becomes, what gradients are important in the atmospheric boundary layer? The work of Klewicki et al. ${ }^{[9]}$ is particularly insightful here. It suggests that the typical assumptions that would be applied to a turbulent boundary layer should also hold for the ABL at least in a local sense (given the length and time scales relevant to wind turbines). The primary of these assumptions is that the horizontal gradient, $\partial / \partial x$, is significantly smaller that the vertical gradient, $\partial / \partial y$. Given that (3.5) describes the pressure on the ground, the result is that the horizontal gradients in the surface pressure fluctuations are dependent on the vertical gradients in the velocity.

The theory supports that primary hypothesis, and indeed many researchers in the field of ABL turbulence hold to these principles in a local sense. Many of the comments of Klewicki et al. ${ }^{[9]}$ reflect this. Nevertheless, in order to turn this into a useable result for a deployable system, either the solution to Navier-Stokes equation must be found or an empirically based model for the relationship between the wavelength, $\lambda$, of pressure fluctuations on the ground and the altitude, $h$, of the related turbulent eddies must be determined. Priestly ${ }^{[18,19]}$ endeavored to develop just such a relationship but did not find good agreement in all atmospheric conditions and was hampered by a lack of velocity data in the ABL. Here, the Task 2 efforts were planned to acquire a data set capable of revisiting Priestly's results with higher fidelity data.

\subsection{Shields' Analysis of Past Infra-Sound Data}

A collaboration with Dr. F. Douglas Shields was formed to provide Dr. Shields the opportunity to re-analyze infra-sound array data collected in Piñon Flat, CA, in September and October of 2007. This new analysis would focus specifically on determining wind speed from correlation-based analysis of the array signals. In previous work, the multi-element array was used to eliminate wind noise through averaging, so attempting to use the wind noise for direct measurement is a new approach. 


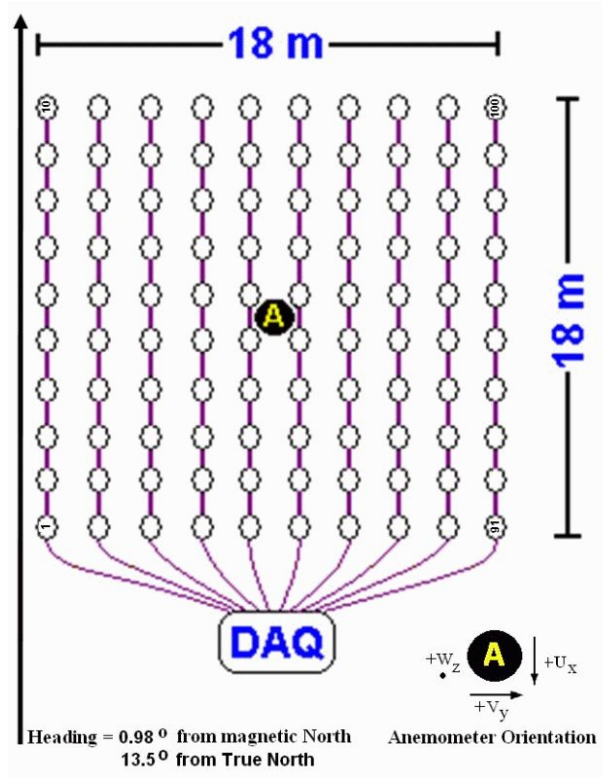

Fig. 3.4. Square array setup in Pinon Flat, CA, from 2007 infra-sound experiments.

The Piñon Flat data was obtained with a square array of 100 elements as shown in Fig. 3.4. The anemometer in the center of the array was only 2.5 meters above the ground which is not at an elevation significant for wind turbines. Nevertheless, the anemometer does give some indication of the wind speed. For reference, Figures 3.5 shows the wind speed and array-averaged pressure for a 15 min. segment of time.

The new analysis of the Piñon Flat data yielded favorable results that support the hypothesis that an array of pressure transducers can be used to predict the wind velocity profile. Dr. Shields was able to apply some new correlation analyses to array data. An example of his results is shown in Fig. 3.6 which plots the correlation derived convection velocity as a function of frequency. The bold red symbols/line is an average of the other plotted data: these represent 4 different 10 minute segments of data (see Appendix B for details). The red ' + ' is the wind velocity measured by an anemometer positioned at the center of the array at $2.5 \mathrm{~m}$ above the ground.

Theoretically, the correlation between signals at lower frequency should be related to physical events with larger wavelength (or correlation length scale). This would imply a relationship between increasing altitude with decreasing frequency. Fig. 3.6 shows an increase in convection speed with decreasing frequency which fits with expectations of a boundary layer flow: velocity is higher farther from the bounding surface. Dr. Shields complete report is provided in Appendix B.

\subsection{Details of Lab Experiments and Field Deployments}

This section presents the details of the various experiments and field tests that were conducted under Task 2. Data analysis and results are presented in later sections. 


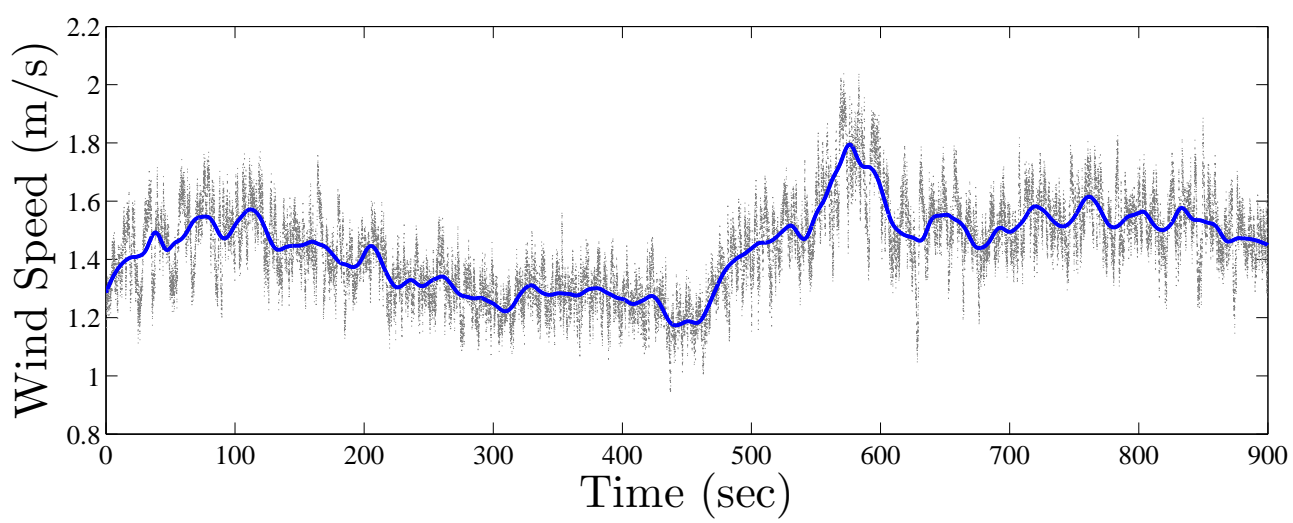

(a) Wind Speed

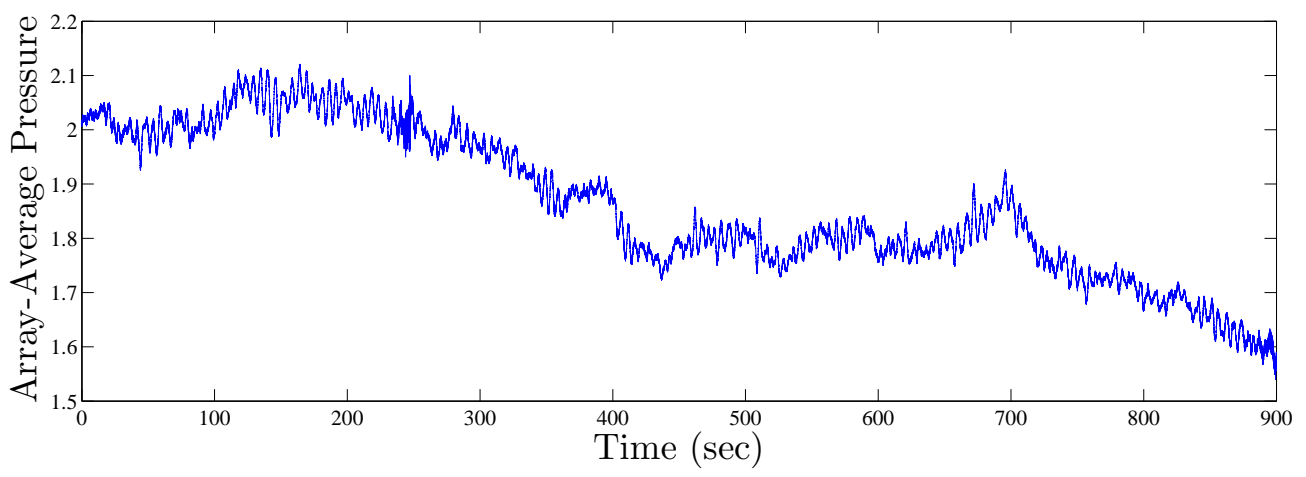

(b) Array-Averaged Pressure

Fig. 3.5. Wind speed and array-averaged pressure during a 900 second sample on 10/1/2007 beginning at $7: 16: 02 \mathrm{pm}$.

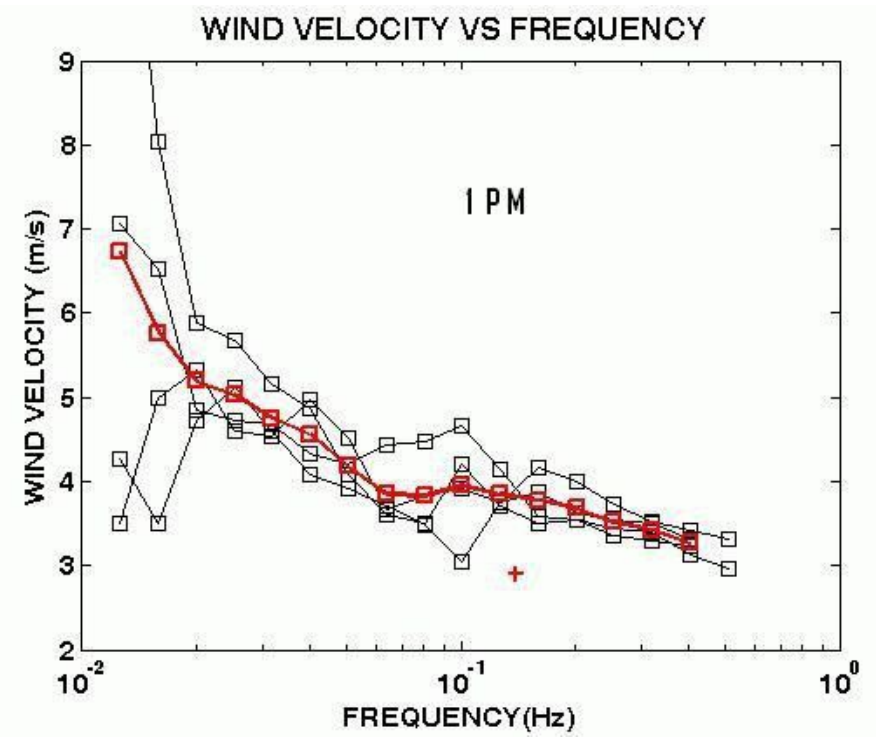

Fig. 3.6. Plot of correlation derived convection velocity as a function of frequency. 


\subsubsection{Low-Speed Wind Tunnel Measurements}

Given that the approach to passive acoustic in-flow profiling is an empirically derived estimation based on surface pressure correlations, an understanding of the effect of surface temperature on the correlations is key. Experiments in the NCPA $2^{\prime} \times 2^{\prime}$ low-speed wind tunnel were carried out to (a) develop an empirical velocity profile estimation methodology and (b) evaluate the effect of surface temperature variation. This section describes the experimental setup and the characteristic data for the wind tunnel environment. Further data analysis is presented in Section 3.6.

The $2^{\prime} \times 2^{\prime}$, low-speed tunnel can reach a maximum test section velocity of approximately $50 \mathrm{mph}$ and has a total test section length of $8 \mathrm{ft}$. An initial scaling analysis confirmed that it is not possible to produce a turbulent boundary layer in the test section whose characteristics are scalable to atmospheric boundary layer (ABL) levels. The drastic difference in Reynolds numbers is too great to overcome. This is a well known issue when trying to simulate ABL flows in a laboratory environment. However, the primary objective in the current work is to predict the velocity at a given elevation using ground-based sensors. The physical basis behind this estimation relies on turbulent boundary layer physics in general. Therefore, the approach was to generate a thick turbulent boundary layer that includes large scale coherence in the wind tunnel environment so that the wall-pressure/flow-field correlations can be studied.

To generate the thick boundary layer, a $3^{\prime \prime}$ back-step geometry was chosen. The back-step was placed immediately at the entrance to the test section. It is known that the reattachment point lies approximately 6 step heights downstream, and that after approximately 20 step heights the flow has regained a large portion of its boundary-layer self-similarity but still retains influences from the large-scale motion generated by the flow separation. ${ }^{[11]}$ For the $3^{\prime \prime}$ back-step, 20 step heights is $5 \mathrm{ft}$. This leaves a $3 \mathrm{ft}$. length in the test section in which measurements were made.

Fig. 3.7 shows the tunnel setup which includes a 3-inch backstep leading into the 8foot long test section. The test section has a 2-foot square cross-section. Tempco silicone rubber coated electric heaters were installed on the test section floor to allow for a heat flux boundary condition. K-type thermocouples were mounted in the vicinity of the heaters and a computer controlled solid state relay was used to supply power to heaters and allow for thermostatic control. During testing with elevated temperature the heater pads were held constant to within $\pm 1^{\circ} \mathrm{C}$.

Eight 1/4 inch Panasonic series WM-61A microphones spaced out along the centerline of tunnel test section were used to acquire the dynamic pressure data. The sensors had a nominal sensitivity of $150 \mathrm{~V} / \mathrm{psi}$ and a specified frequency range of 20-20,000 $\mathrm{Hz}$. The microphones were placed in and flush mounted with a top surface of a thin strip of wood that allowed for alignment and running the microphone wires without having to cut through and possibly disable to heating strip on which it was mounted. Photographs illustrating the trimmed wood piece placed on top of the orange heat strips inside the wind tunnel test section, along with the microphones, are presented in Fig. 3.8. Microphone number 1 was placed $1.61 \mathrm{~m}$ downstream of the backstep inlet. Subsequent microphones were placed 0.006, $0.019,0.044,0.095,0.197,0.400$, and $0.806 \mathrm{~m}$ downstream of microphone 1 . The variation in sensor spacing was chosen to allow for a range of correlation lengths to be investigated. 


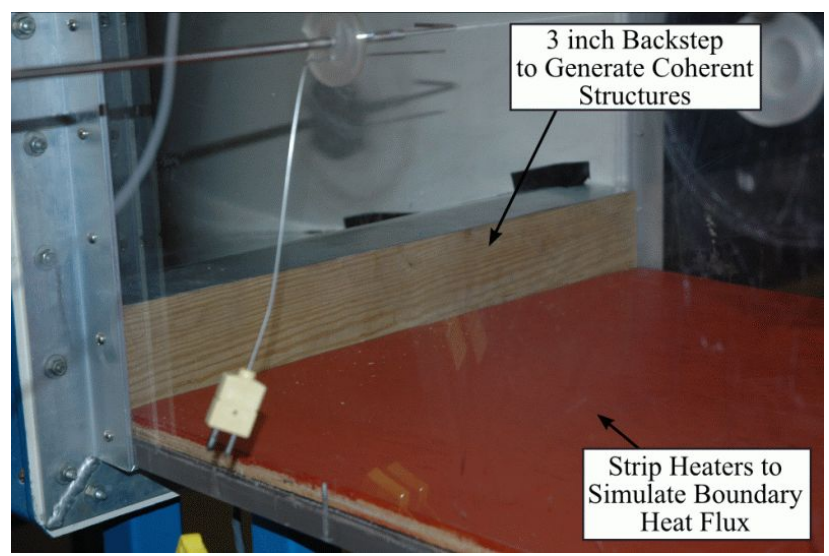

Fig. 3.7. Low-speed wind tunnel inlet modified with a $3^{\prime \prime}$ backstep at the test section entrance.

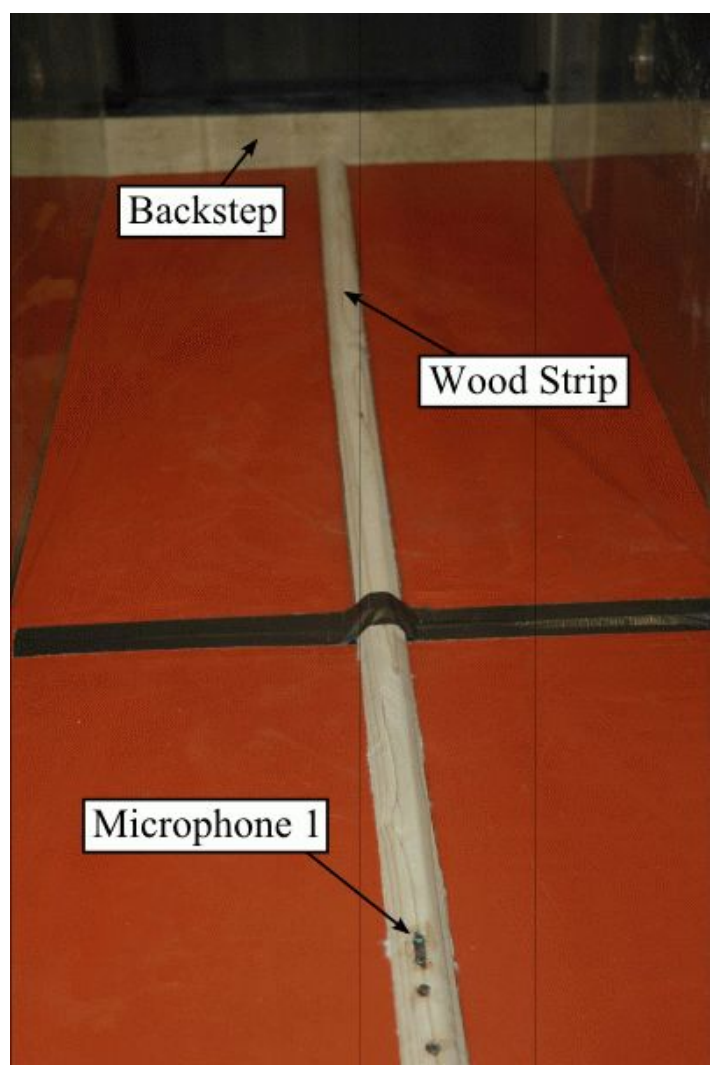

(a) Backstep and Sensor Strip

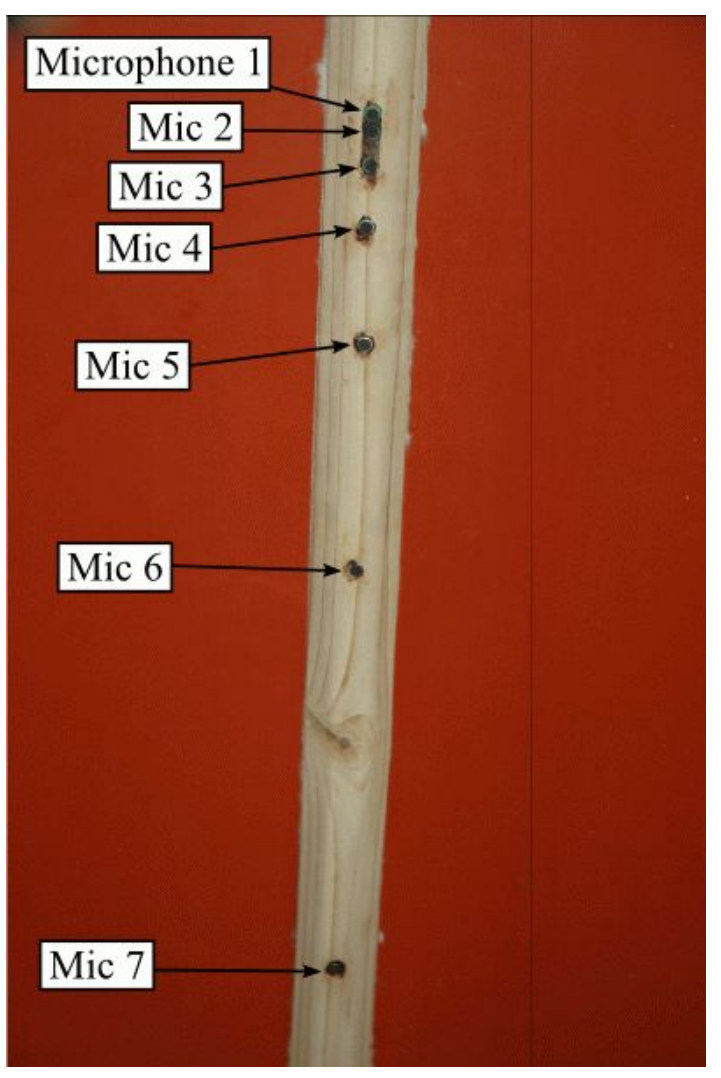

(b) View of Sensor Spacing

Fig. 3.8. Trimmed wood piece installed on top of the strip heaters along the centerline of the wind tunnel test section floor with microphones flush mounted in the downstream section of it. 


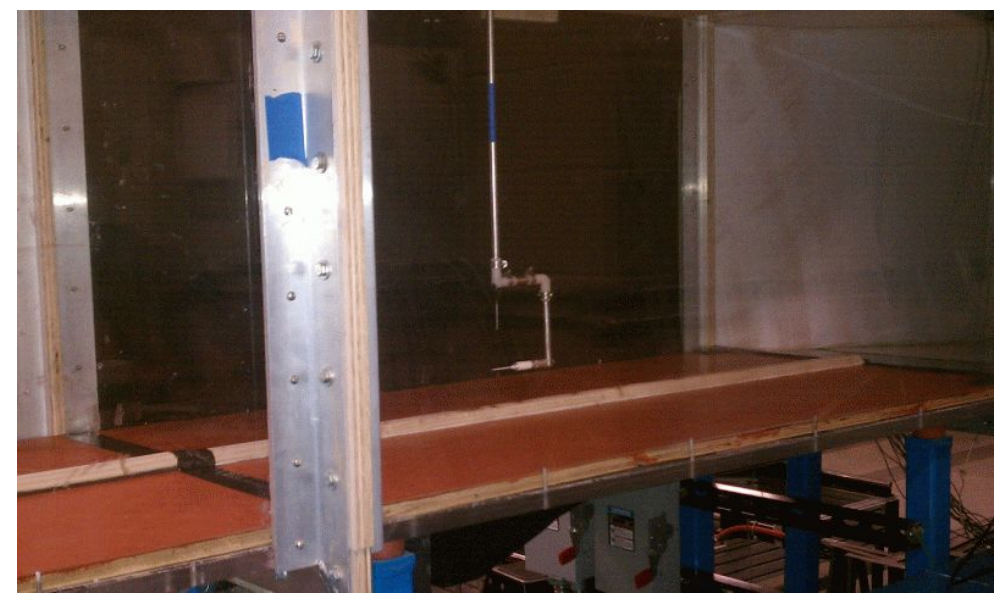

Fig. 3.9. Photograph of the hot-wire probe within the wind tunnel test section.

A two-wire hot-wire probe connected to a Dantec CTA system and attached to a traverse system was used to acquire the velocity measurements above select microphones. A photograph of the hot-wire probe above the strip with microphones is shown in Fig. 3.9. The rod attached to the probe was mounted on a traversing system located on the top of the tunnel which allowed for horizontal and vertical movement. The hot-wire measurements were taken at 39 locations throughout the vertical extent of the wind tunnel boundary layer above select microphone locations starting at approximately $0.013 \mathrm{~m}$ above the floor. The next 14 measurements were acquired every $0.006 \mathrm{~m}$ and the remaining 24 measurements were spaced out every $0.013 \mathrm{~m}$ until the last measurement was acquired $0.406 \mathrm{~m}$ above the test section floor. The horizontal component of the velocity acquired with the hot-wire at vertical location of $0.406 \mathrm{~m}$ above the heater strips was considered to represent the freestream velocity $\left(U_{\infty}\right)$ of the flow within the tunnel.

The 2D hot-wire probe was used to measure the streamwise, $U$, and vertical, $V$, components of the velocity. In the following the mean velocity is denoted with a captial $U$ while the fluctuating part is denoted with lower-case, $u$. Calibration measurements were made in situ as shown in Fig. 3.10 where the hot-wire was placed at various angles to the freestream flow and acquired voltages were compared to the velocities determined by a reference pitot probe.

The hot-wire data was collected synchronously with the microphone data at several freestream velocities, various surface temperatures, and at different longitudinal locations in order to define the effect of flow speed and surface temperature on the measured turbulence and predict downstream flow speed based on upstream pressure measurements. In particular, the measurements were acquired at tunnel (freestream) velocities, $U_{\infty}$, of $6.1,7,8,9$, and 9.5 $\mathrm{m} / \mathrm{s}$, with surface temperatures, $T_{s}$ of $23,32,50$, and $65^{\circ} \mathrm{C}$. Measurements were made at six longitudinal locations corresponding to microphones 1 through 6 , and 39 vertical locations as noted above

The spectra of the hot-wire measured streamwise, $u$, and vertical, $v$, velocity components of select run at various heights is shown in Fig. 3.11 for a freestream velocity, $U_{\infty}=7.9 \mathrm{~m} / \mathrm{s}$ and surface temperature, $T_{s}=23^{\circ} \mathrm{C}$ (unheated). Moreover, the influence of tunnel speed 


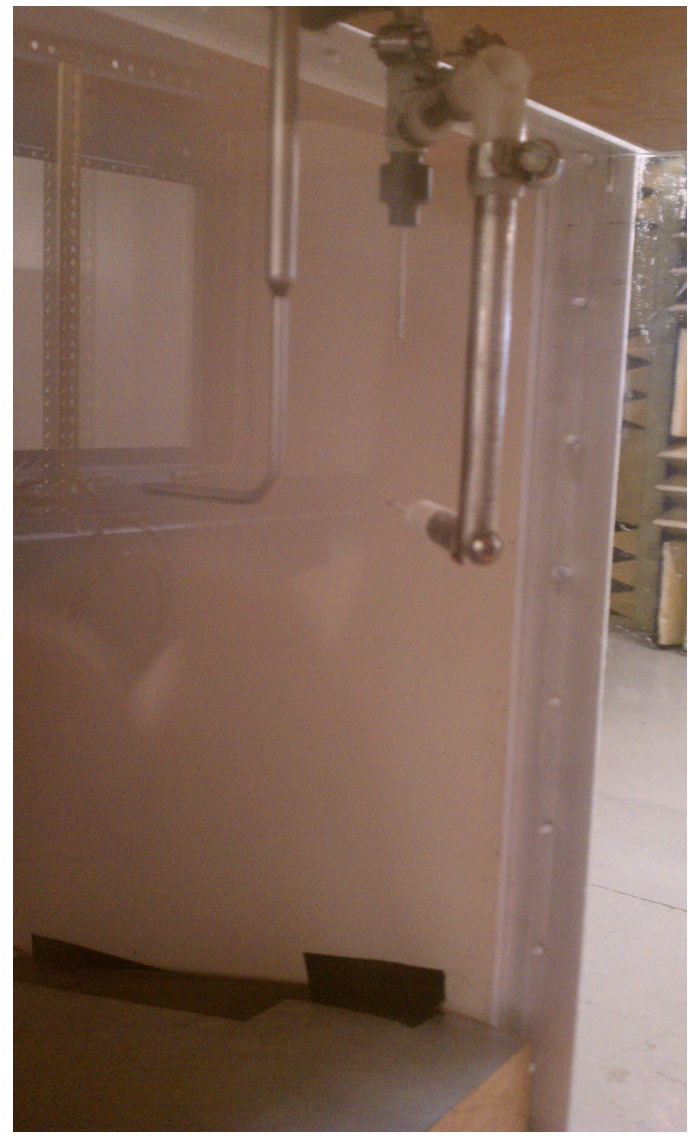

(a) Hot-Wire and Pitot Probe

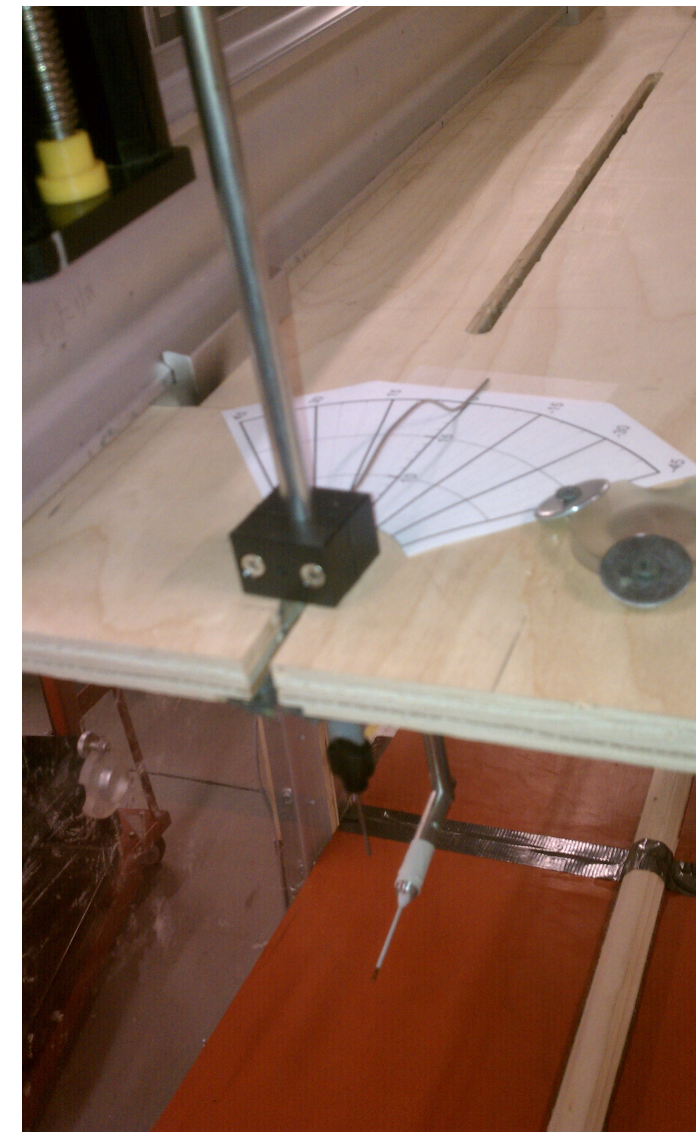

(b) Compass Indicator for Setting Angle

Fig. 3.10. Photographs of the calibration setup where the hot-wire probe is angled to the freestream flow and acquired voltage values are compared to those from a pitot probe. 


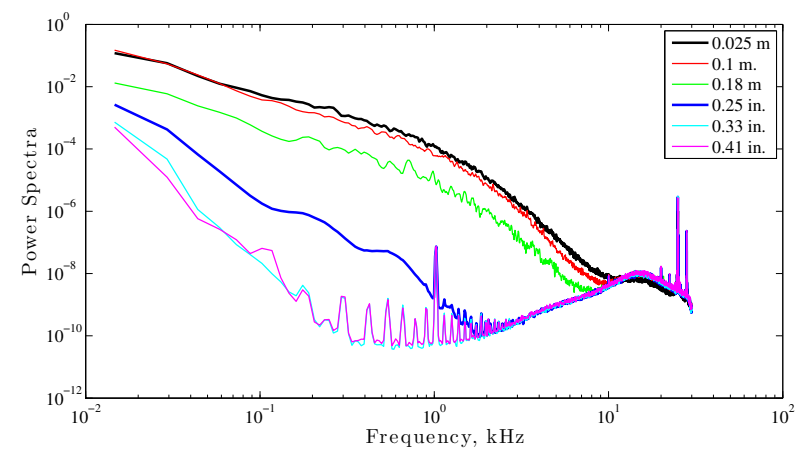

(a) $u$-velocity

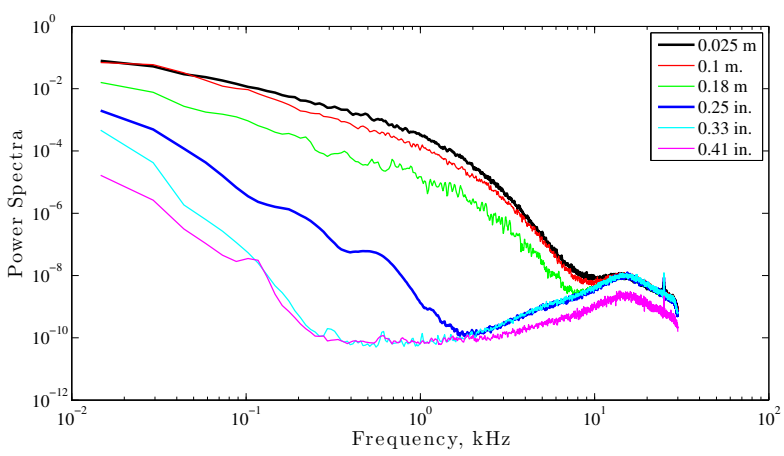

(b) $v$-velocity

Fig. 3.11. Spectra of the $u$ and $v$ velocity components measured with a hot-wire located over microphone 4 at various heights, surface temperature of $23^{\circ} \mathrm{C}$ and freestream speed of $7.9 \mathrm{~m} / \mathrm{s}$.

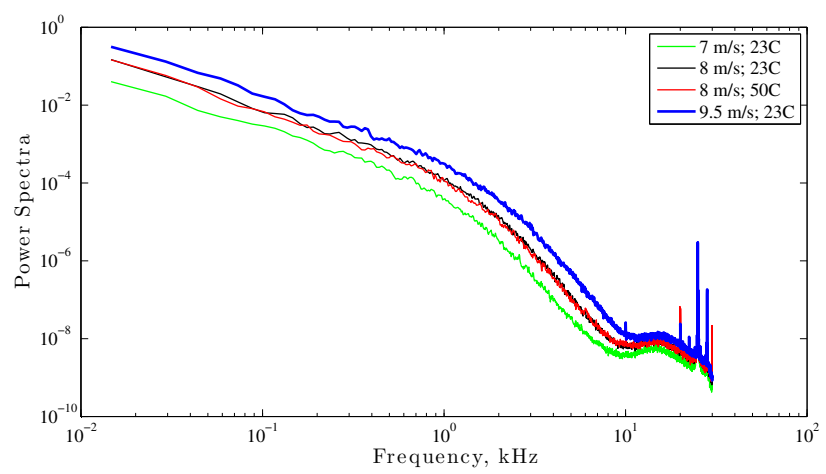

Fig. 3.12. Spectra of the u velocity component measured with a hot-wire at a height of $0.05 \mathrm{~m}$ above the microphone 4 for runs with different freestream speeds and surface temperatures.

and surface temperature on the $u$ spectra at $h=0.05 \mathrm{~m}$ is shown in Fig. 3.12. For this range of $U_{\infty}$ and $T_{s}$ the turbulence spectra seems relatively insensitive to surface temperature.

The mean velocity profiles of the $U$-velocity above various select microphones are illustrated in Fig. 3.13 for $U_{\infty} \approx 8 \mathrm{~m} / \mathrm{s}$. Self-similarity is demonstrated here as the profiles are consistent over the 0.2 meter span between microphones 2 and 6 . The effect of surface temperature on the resulting mean velocity profile above microphone 4 is shown in Fig. 3.14 which shows negligible dependence on $T_{s}$ over the range tested. Fig. 3.15 shows the dependence of the mean $U$ velocity profile on the freestream $U_{\infty}$.

The effect of tunnel speed, $U_{\infty}$, and surface temperature, $T_{s}$ at a given longitudinal station in the wind tunnel on turbulence levels can be represented by computing the RMS values, $\sqrt{\overline{u^{2}}}$, the mean kinetic energy, $k=0.5\left(\overline{u^{2}+v^{2}}\right)$, or the turbulence intensity level, $\sqrt{2 k / 3} / U_{\infty}$. All of these represent similar quantities. Here, the kinetic energy, $k$, is plotted for several $U_{\infty}$ and $T_{s}$ in Fig. 3.16. While it is obvious that $U_{\infty}$ has the largest effect on the turbulence levels, it is also evident that the increase in $T_{s}$ does slightly reduce the maximum $k$ in the boundary layer. That is, the turbulence intensity is inversely proportional to the surface temperature over this range. This may be counter-intuitive; however, the increase 


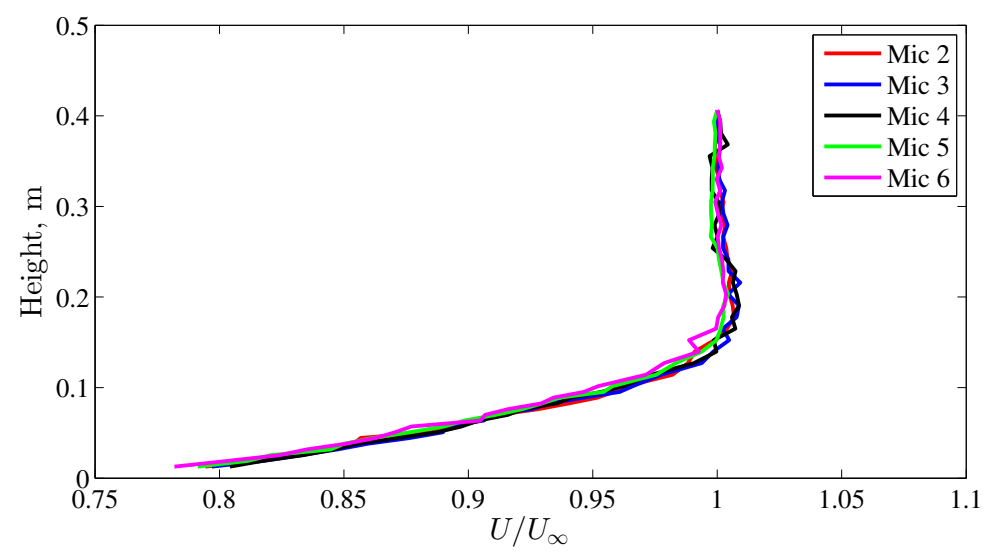

Fig. 3.13. Mean velocity profiles of hot-wire measurements above various microphones for runs with surface temperature of $23^{\circ} \mathrm{C}$ and tunnel speed of approximately $8 \mathrm{~m} / \mathrm{s}$.

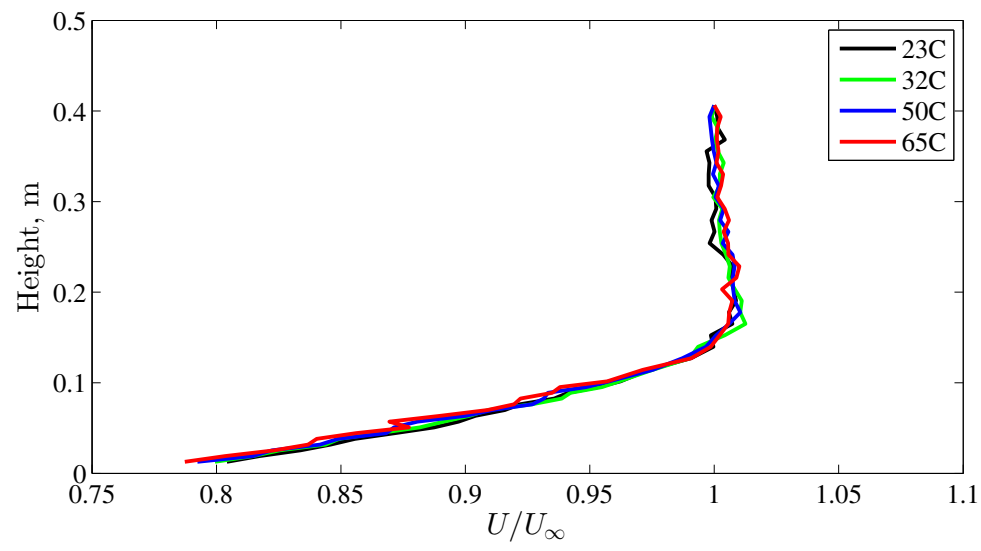

Fig. 3.14. Mean velocity profiles of hot-wire measurements from runs with various surface temperatures and tunnel speed of approximately $8 \mathrm{~m} / \mathrm{s}$.

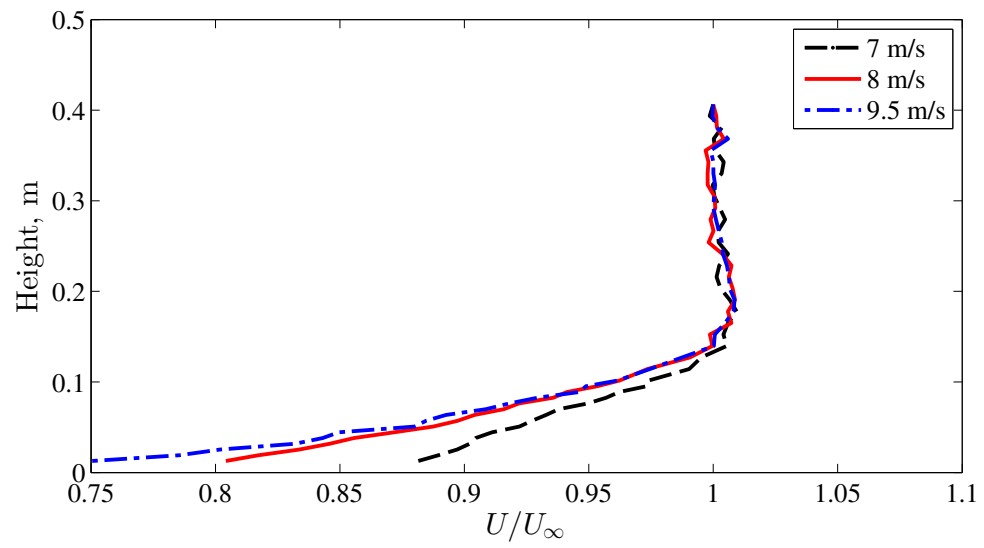

Fig. 3.15. Mean velocity profiles of hot-wire measurements from runs with various tunnel speeds and surface temperature of $23^{\circ} \mathrm{C}$. 


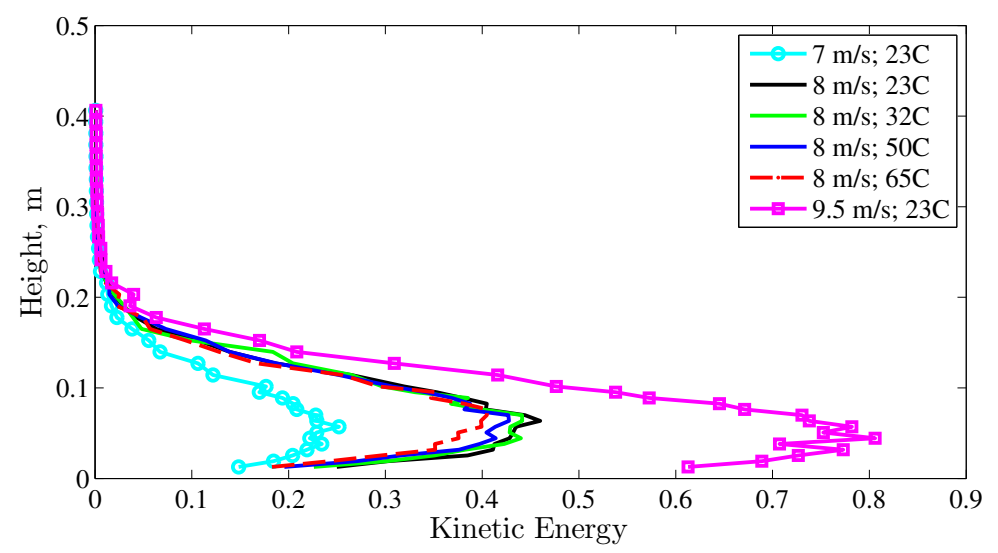

Fig. 3.16. The effect of surface temperature and tunnel flow speed on the kinetic energy computed from the two measured velocity components.

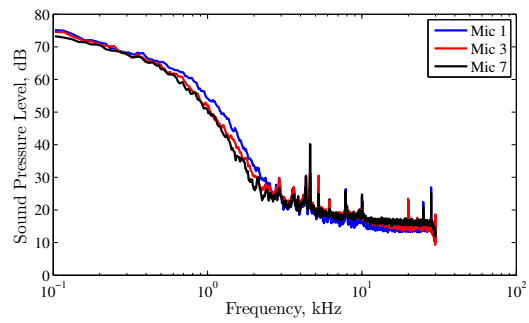

(a) Microphone Location

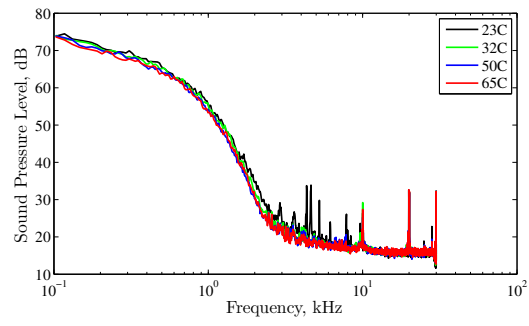

(b) Temperature

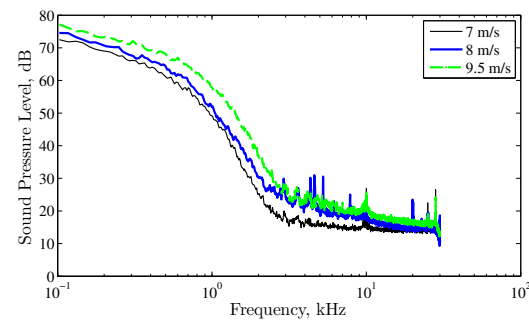

(c) Freestream Velocity

Fig. 3.17. The effect of microphone location, temperature, and freestream velocity on the sound pressure level values.

in $T_{s}$ serves to add buoyancy to the fluid which should spread the energy out in the vertical direction thereby reducing the absolute maximum value.

Pressure measurements were acquired synchronously with the hot-wire data. Although some variation was noted in pressure measurements as the hot-wire height was changing, the analysis of the pressure data was carried out only on measurements acquired when the hot-wire was at its furthest point away from the microphones (height of $0.41 \mathrm{~m}$ ). Here the general characteristics are presented. Further correlation-based analysis to estimate the velocity profile is presented later in Section 3.6.

The analysis of pressure measurements was initiated by looking at the sound pressure level (SPL) values acquired during the runs. Fig. 3.17 illustrates the small variation in SPL results depending on (a) which microphone in a given run was considered, (b) the effect of temperature on the resulting SPL values for runs with similar freestream speeds and longitudinal location, and (c) the effect of freestream speed on the SPL values for runs with the same surface temperature and longitudinal location. In all, only the $U_{\infty}$ variation causes a measurable effect on the SPL. 


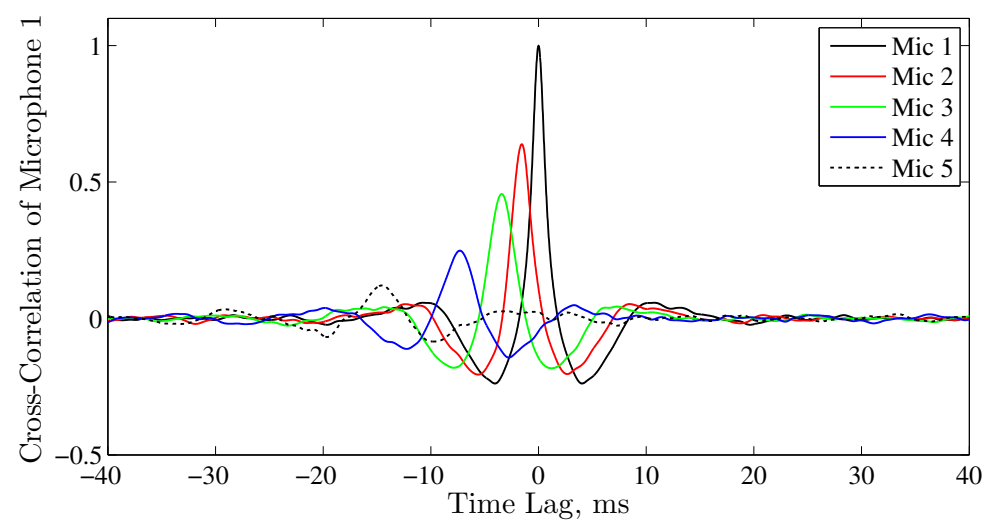

Fig. 3.18. Cross-correlation of microphone 1 with other microphones for a sample run at room temperature.

The cross-correlation coefficients between the eight pressure sensors were calculated as

$$
\rho_{p_{i}^{\prime}, p_{j}^{\prime}}\left(x, \xi_{x}, \tau\right)=\frac{\overline{p_{i}^{\prime}(x, t) p_{j}^{\prime}\left(x+\xi_{x}, t+\tau\right)}}{p_{i, r m s}(x, t) p_{j, r m s}\left(x+\xi_{x}, t+\tau\right)}
$$

using Matlab's build-in xcorr function, which provided the means to compute a convection velocity and an integral time scale for the surface pressure signals. Microphones 7 and 8 were found to be located too far from the other microphones and no cross-correlation was found. Sample results for $\rho_{p_{1}^{\prime}, p_{j}^{\prime}}$ of microphone 1 with other microphones at $U_{\infty}=8 \mathrm{~m} / \mathrm{s}$ and $T_{s}=23{ }^{\circ} \mathrm{C}$ are plotted in Fig. 3.18 showing the characteristic time shift and decay of $\rho_{\operatorname{MAX}} \cdot{ }^{[2]}$

\subsubsection{Field Experiments}

\subsubsection{Locke Station, MS, November 2010}

The first significant field experiment was performed over the course of 5 days at a location near Locke Station in the Mississippi Delta. Fig. 3.19 shows a Google Earth view of the test site whose GPS center is at $34.26453^{\circ} \mathrm{N}, 90.20182^{\circ} \mathrm{W}$. A large array of 49 ground-based measurement locations covering a circular area with a 400 meter diameter was populated with two different types of pressure sensing elements at each measuring location. Fig. 3.20 shows the layout of the sensor elements. These elements recorded their data continuously from 1:00 pm Monday, November 8 through 8:00 am Friday, November 12.

A SODAR unit was deployed on site to record data simultaneously with the array over a 24 hour period starting Tuesday at 2:00 pm. The SODAR was placed just South-West from the array at the location shown in Fig. 3.19. Fig. 3.21 shows the deployed SODAR system. Fig. 3.22 shows a typical time trace of signal returns from the SODAR unit from midnight to 2:00 pm on Wednesday, 10 November.

At the center of the array, a sonic anemometer was placed at a height of 124 inches giving the wind vector near the ground at the array origin. Fig. 3.23 is a view looking South-East from the center of the array showing the anemometer position. The field site had recently been cleared and was characterized by very regular soil rows as shown in Fig. 3.23. Fig. 3.24 shows the wind velocity and temperature measured by the anemometer during the course of 


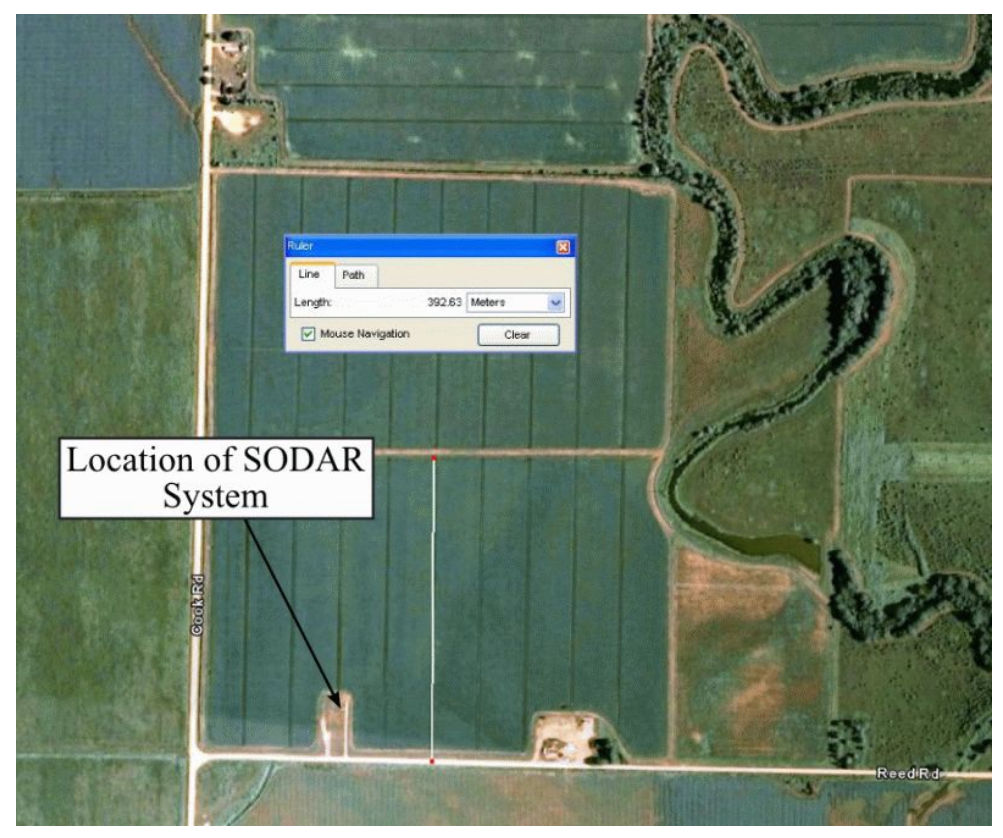

Fig. 3.19. Google Earth view of test site at the corner of Reed and Cook Roads, Locke Station, MS: GPS center $34.26453^{\circ} \mathbf{N}, 90.20182^{\circ} \mathbf{W}$.

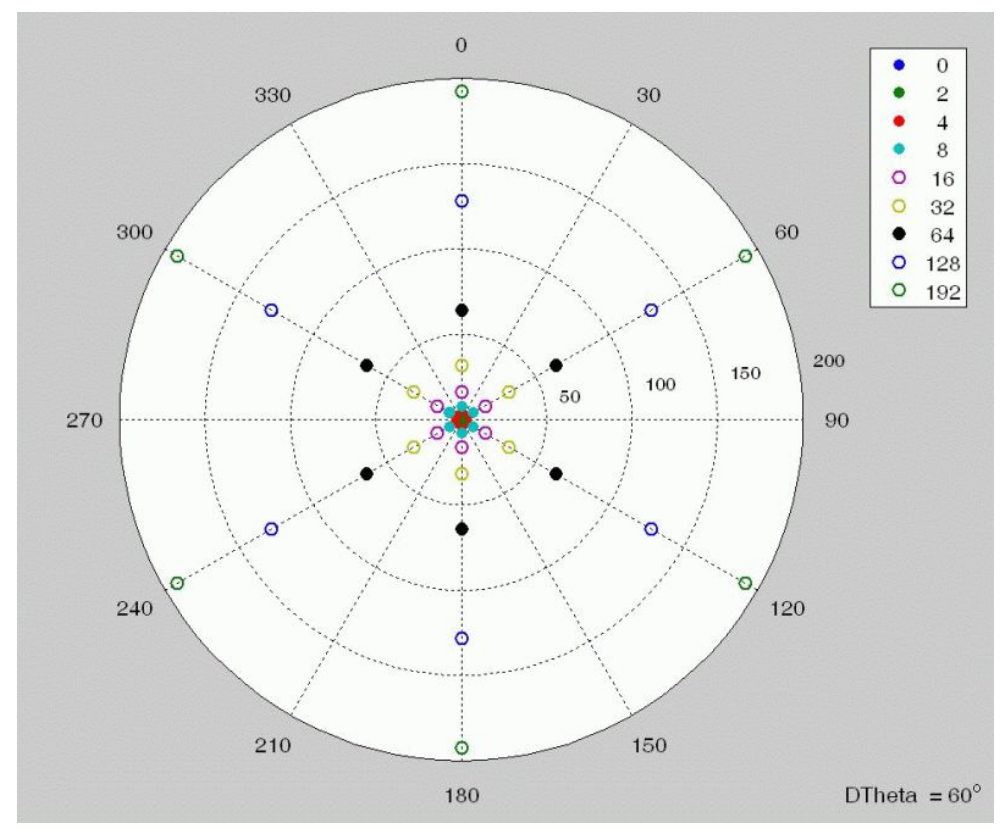

Fig. 3.20. Layout of infra-sound sensor array at the Locke Station test site. Six arms with 8 sensors in each arm and a single unit at the center giving 49 total sensor locations. 


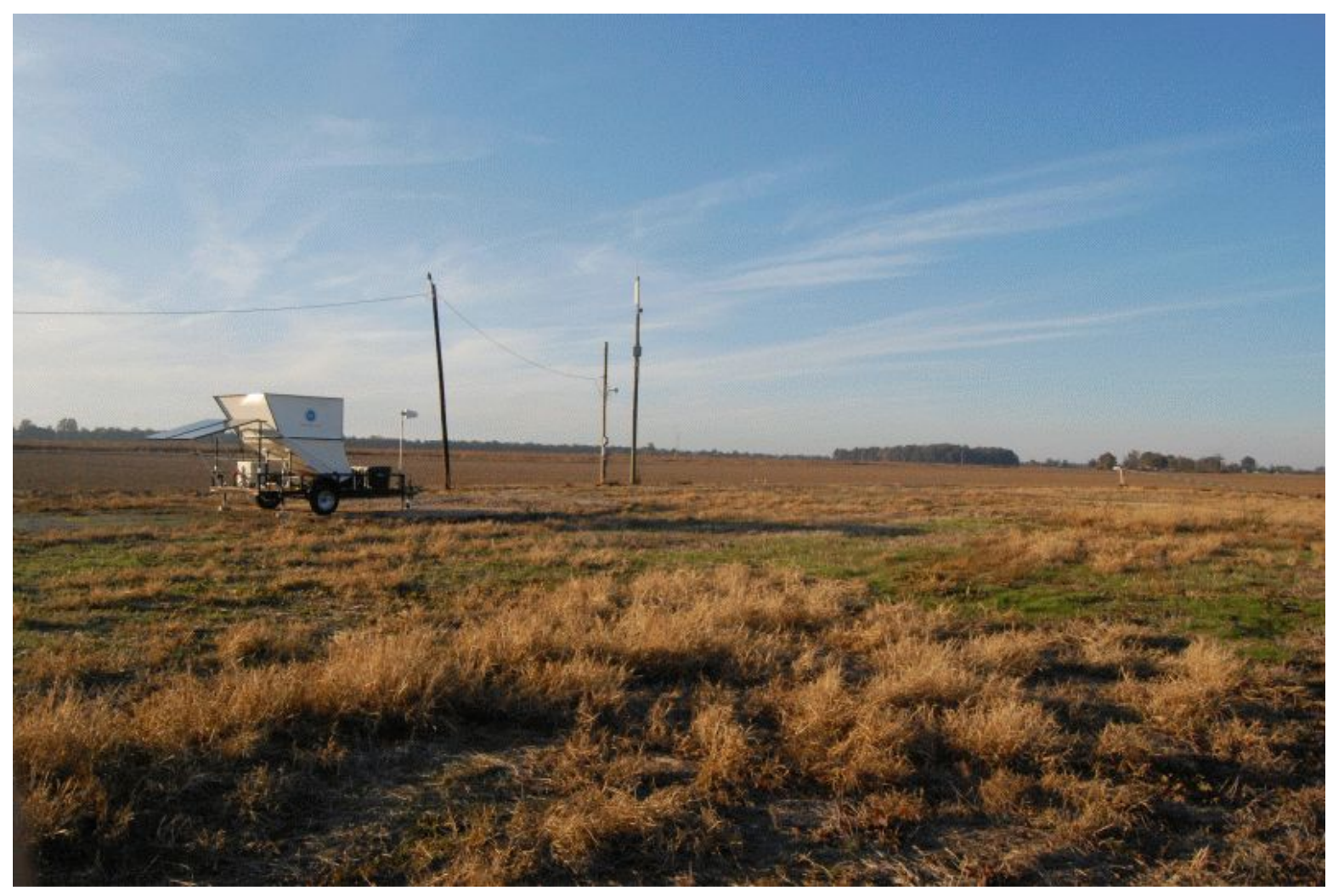

Fig. 3.21. View of the SODAR system deployed at the Locke Station test site.

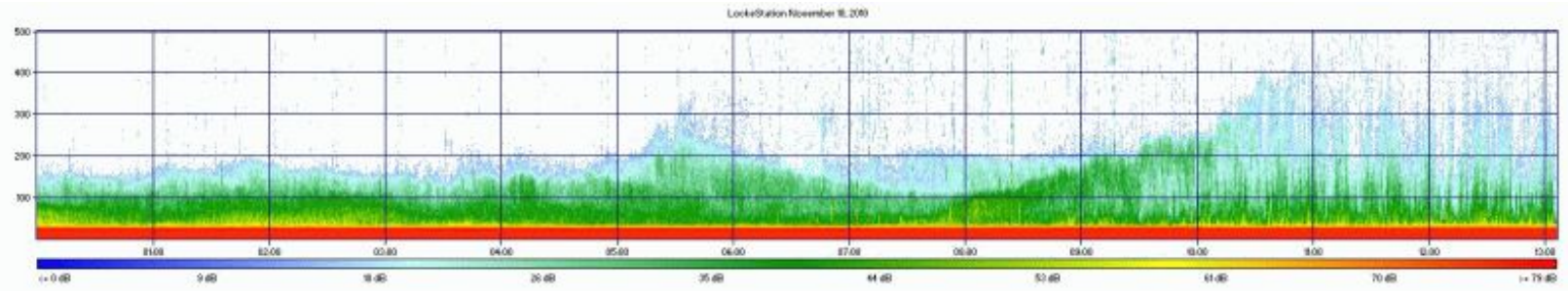

Fig. 3.22. Sample SODAR time trace of signal returns from the Locke Station data: colored by signal strength, plotted versus time on the horizontal and altitude on the vertical. 


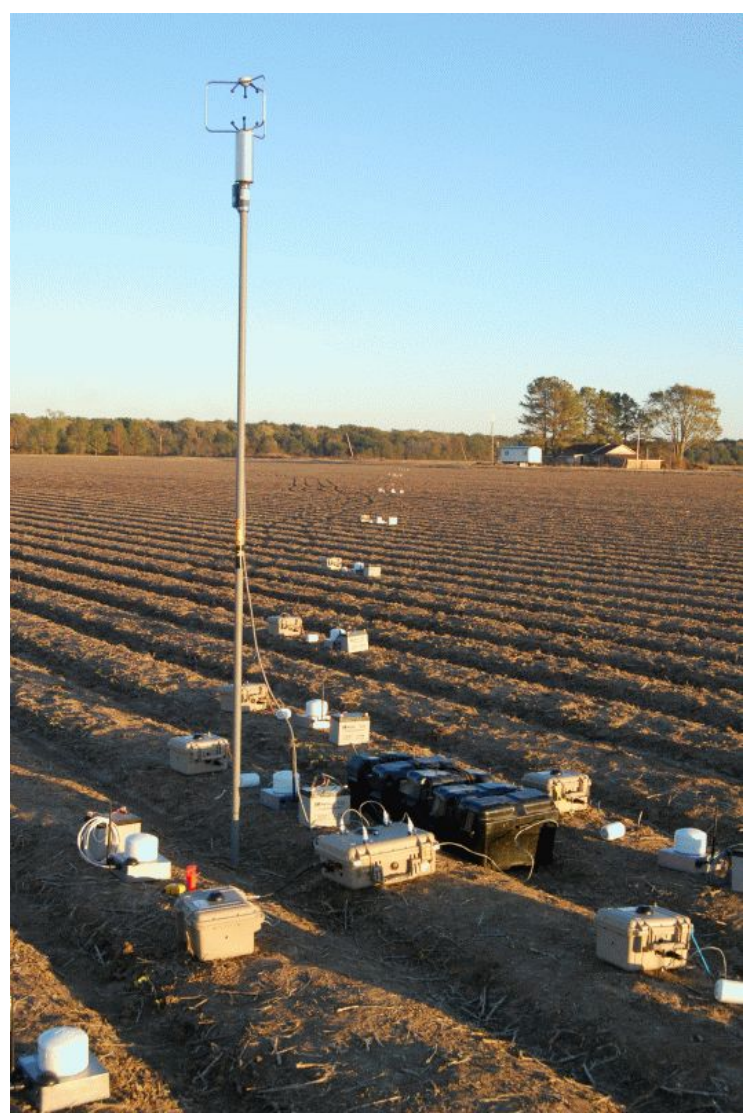

Fig. 3.23. View of the test site looking South-East from the array center. The sonic anemometer shown is positioned 124 inches above the ground.

the experiment. The diurnal cycle is clearly evident and the conditions over the course of the three day deployment were very repeatable. Note that both the mean velocity, $|U|$, and the velocity fluctuations, $u^{\prime}$, increase during daylight hours except on Thursday. This is due to a weather front that passed through on Friday.

The pressure sensor array was populated with two different sensor types at each measurement location. This was specifically to satisfy objective (c) in Subtask 2.2: obtain comparative measurements with infrasound sensors. One sensor was the NCPA developed UMX sensor referred to as NCPA-UMX below, and the other was the Miltec Research \& Technology (MRT) Triple-Threat system referred to as MRT-TT below. Both of these units are shown in Fig. 3.25. The MRT-TT sensing element is derived from the Shields dualbimorph design. ${ }^{[26]}$ Each MRT-TT sensing element was connected with a short umbilical to a power and data acquisition box. The NCPA-UMX sensor is an earlier version of that deployed in the USArray program ${ }^{\ddagger}$ and also uses piezo-bimorphs but in a clover-leaf pattern. ${ }^{[28]}$ Both the MRT-TT and NCPA-UMX sensor systems offer GPS locked stand-alone sensors that record data autonomously and can be accessed wirelessly as needed.

Fig. 3.26 shows a comparison between two collocated sensors over the course of the field deployment. Data was continuously sampled from 6 pm Monday through 6 am Friday. The

\footnotetext{
${ }^{\ddagger}$ http://http://www. usarray.org/
} 


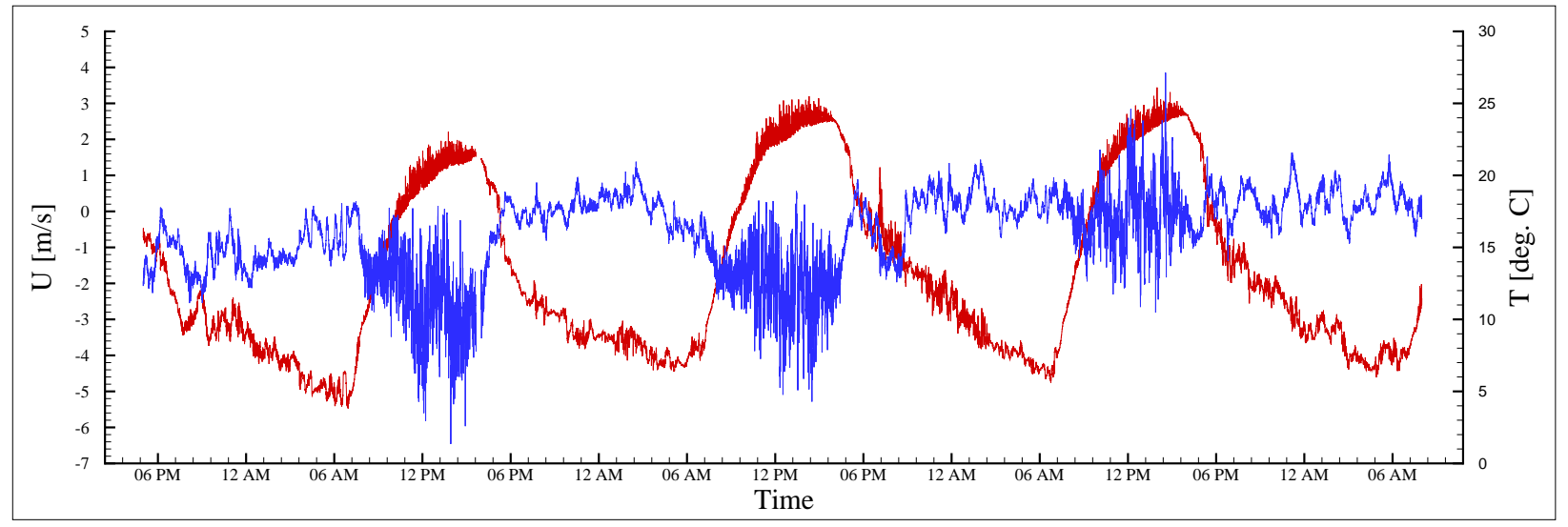

Fig. 3.24. Temperature and horizontal velocity data measured by the anemometer during the Locke Station field deployment. Red is the temperature and blue is the $U$ velocity where $-U$ is the South-to-North direction.

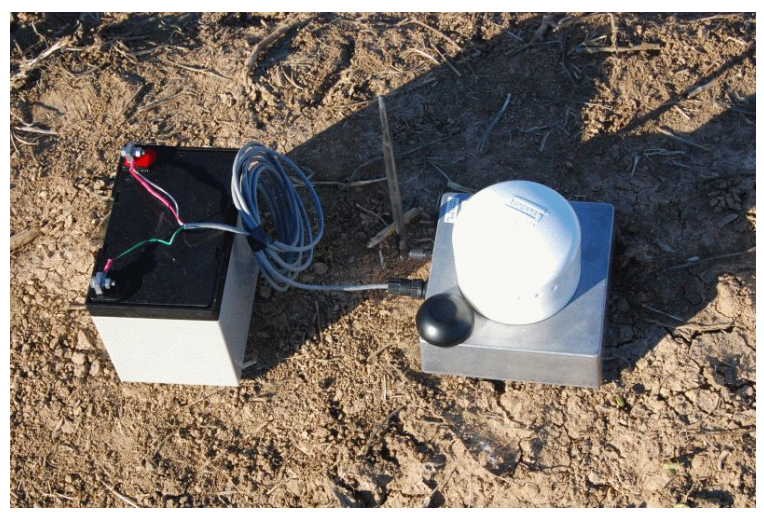

(a) NCPA-UMX Sensor Unit

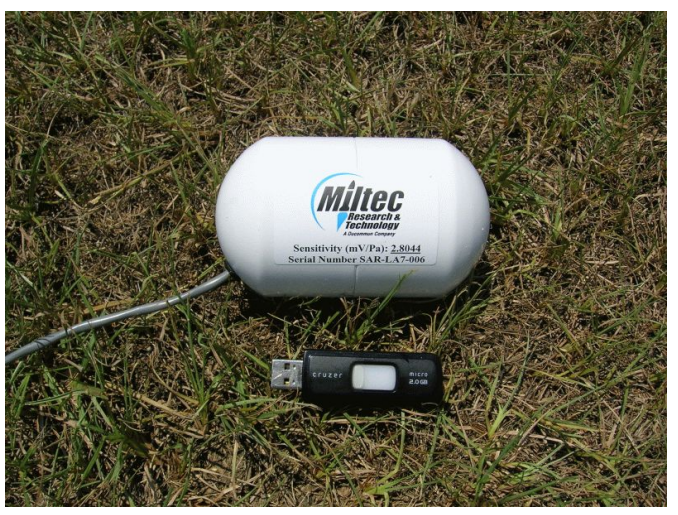

(b) MRT-TT Sensor Unit

Fig. 3.25. Infrasound sensor units used to measure atmospheric pressure fluctuations at the ground. The MRT-TT sensor was connected to an external power supply and data acquisition box which is not shown in the photo. 


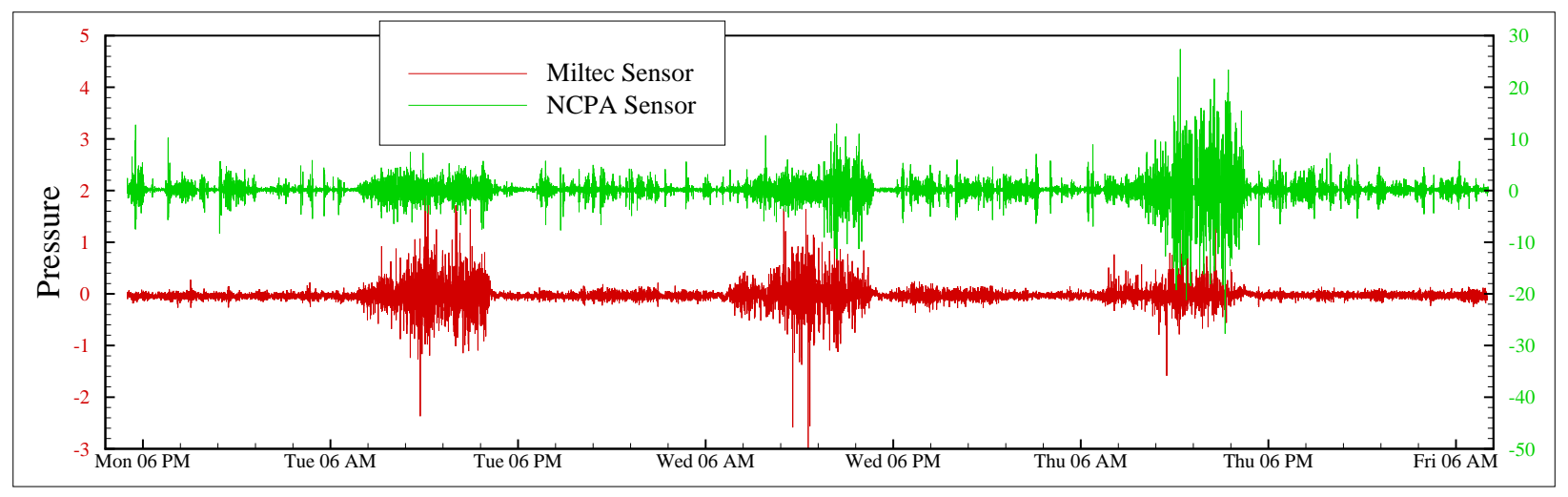

Fig. 3.26. Characteristic pressure signals showing a comparison between the NCPA-UMX and MRT-TT sensor over the duration of the Locke Station field deployment.

characteristic diurnal cycle shows up in the pressure data as an increase in $P_{r m s}$ during daytime conditions. This corresponds to a thicker boundary layer and higher turbulence levels characteristic of unstable atmospheric conditions.

The results shown in Fig. 3.26 were used to compare the capabilities of the two sensor types for future use. The velocity and temperature data in Fig. 3.24 suggest very repeatable conditions over the three days of the deployment. The MRT-TT sensor exhibits a very distinct difference between the day and night RMS levels. In contracts, the NCPA-UMX sensor, located at the same physical position in the deployed array, exhibits unusual bursts of "noise" in the pressure signal during the day. Additionally, the NCPA-UMX sensor's calibration resulted in levels 10 times larger than the MRT-TT sensor. Note that the MRTTT sensor was calibrated to a reference B\&K 4193-L. Based on these results, the MRT-TT sensor was identified as a more reliable measurement. Therefore, the bulk of the remaining analysis was focused on data from the MRT-TT sensors.

Klewicki et al. ${ }^{[9]}$ noted that the single point statistics of the pressure provided some general characteristics of the ABL. In that vein, probability density functions (PDFs) for select segments of the data were computed. The results reflect the increase in $P_{r m s}$ from approximately 0.05 at night to nearly 0.7 during the day. Fig. 3.27 plots the PDFs averaged over a 1 hour time period showing the difference between day and night conditions. The nighttime data was from 00:00 hours and the daytime data was from 12:00 hours local time on Tuesday, Nov. 9, 2010. The same data is plotted in as the Log of the amplitude in Fig. 3.28 which shows the nighttime data exhibits a significant skewness. The negative skewness suggests high-frequency, high-amplitude negative events which would correspond to gusting. ${ }^{[21]}$ This lends support for the ability to detect gusting events using the surface pressure array.

Figures 3.29 and 3.30 show the one-sided auto-spectral density of the pressure signals. The spectra for three individual sensor's signals are shown along with the spectra for a spatially averaged signal obtained by averaging all the array elements together. It is known that the averaging reduces the "wind noise" by $1 / \sqrt{n}$ where $n$ is the total number of sensors in the average. ${ }^{[26]}$ The two spectra show the characteristic differences in the spectra for daytime 


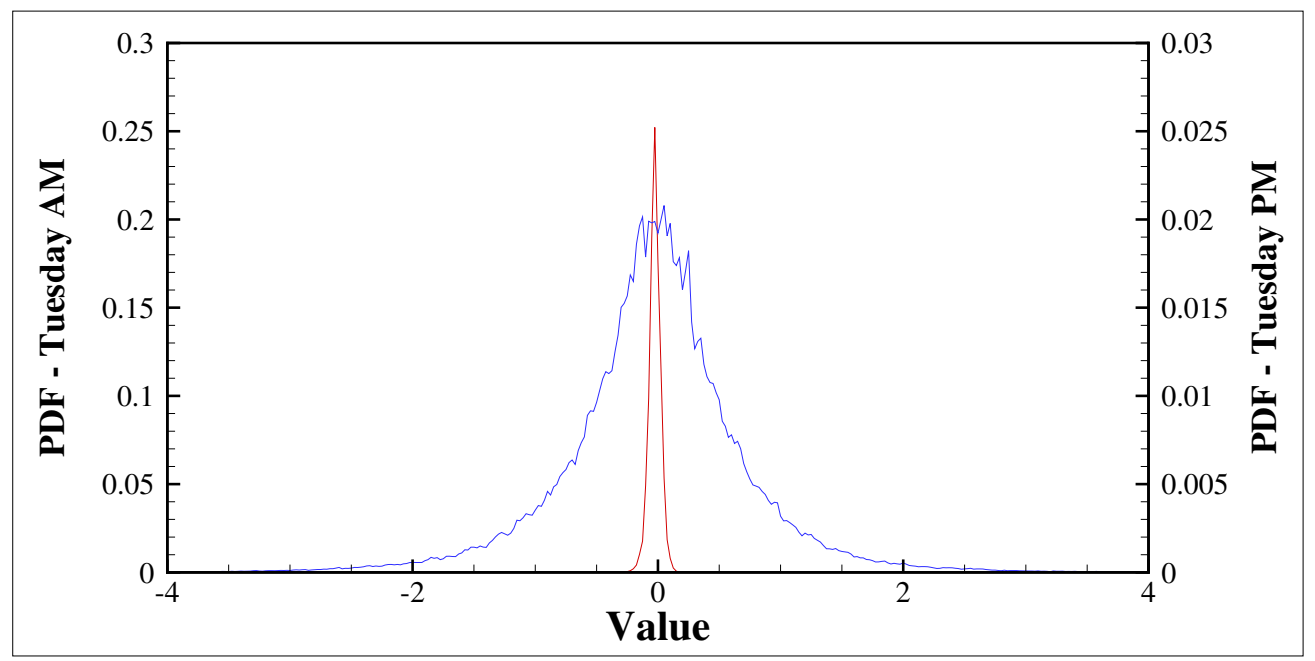

Fig. 3.27. Probability density functions (PDFs) of the pressure fluctuations observed a 1 hour period. Wider curve is the daytime data.

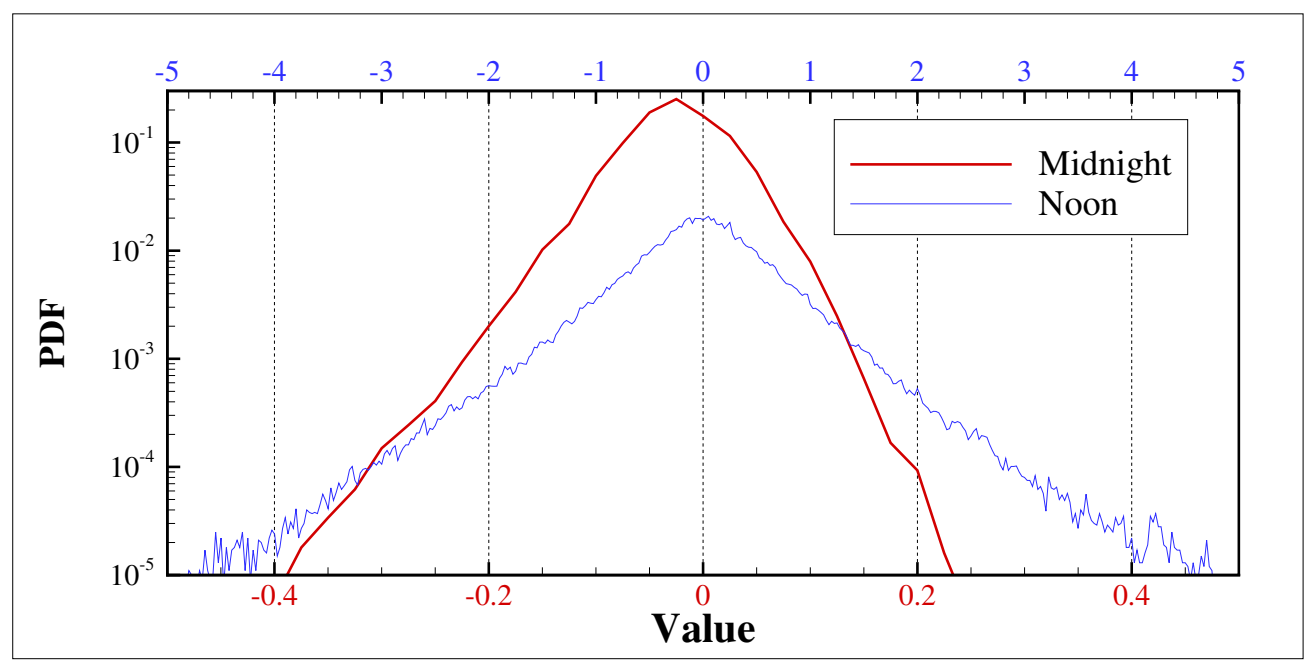

Fig. 3.28. Same PDFs as in Fig. 3.27 but plotted in LOG scale to show skewness. 


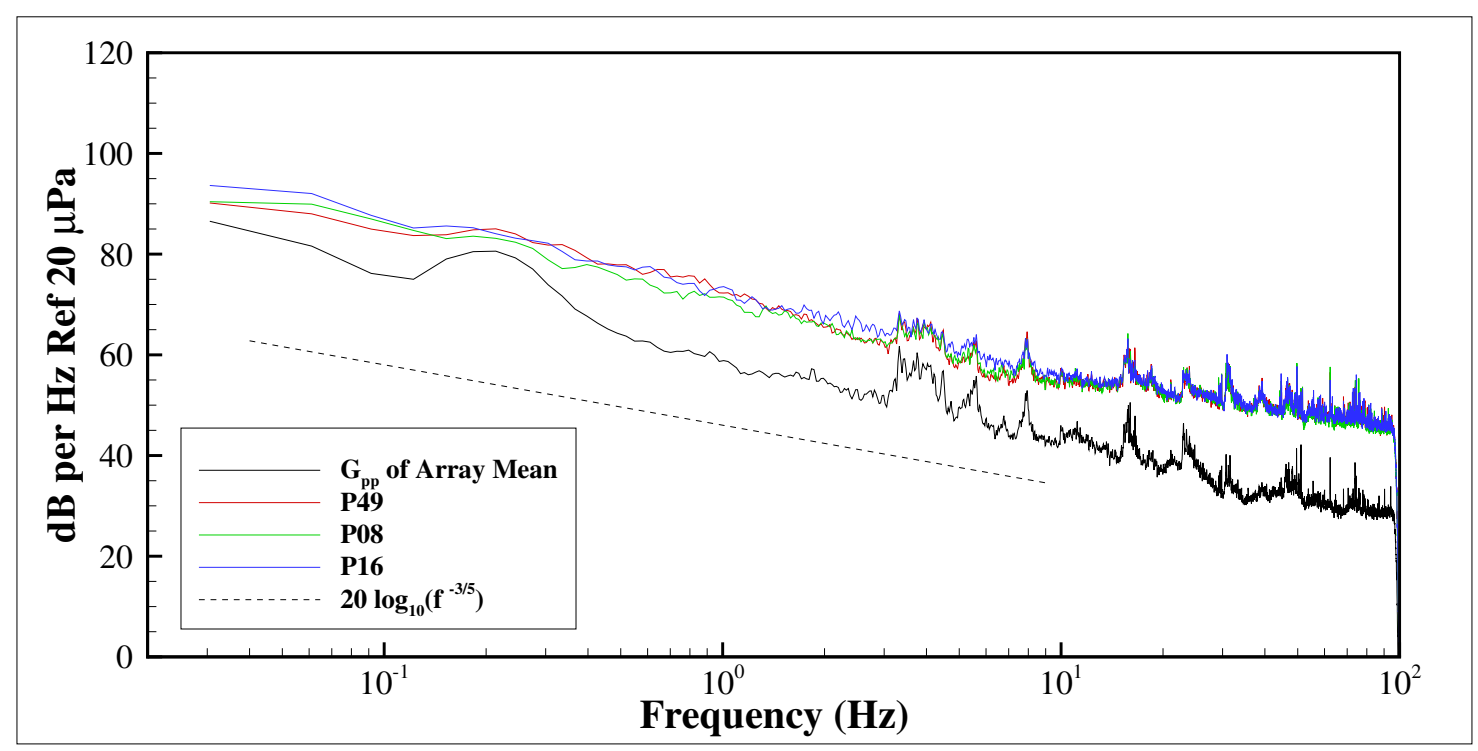

Fig. 3.29. One-sided auto-spectral density of three individual MRT sensors and also of the entire array average signal for Tuesday midnight data.

and nighttime conditions at the site. It is interesting to note that with the array averaging, the wind noise is reduced enough to observe the microbarom signal in the nighttime data. ${ }^{[29]}$

\subsubsection{Reese Technology Center, Lubbock, TX, June 2011}

An field deployment in collaboration with Texas Tech University was conducted at the Reese Technology Center (RTC) in Lubbock, TX, on 21-23 June 2011. This experiment provided coincident measurements of the atmospheric boundary layer with four different measurement systems:

200-meter Met. Tower The 200-meter meteorological tower operated by RTC provided measurements of the wind velocity, air temperature, relative humidity, and barometric pressure at various elevations along its vertical extent. The sampling rate was $20 \mathrm{~Hz}$ for all the data acquired from the tower (see Figures 3.31 and 3.32).

Small Aperture Array A small aperture array of 96 wired MRT-TT pressure sensors was distributed on the ground covering a 60-meter aperture. Data was acquired for all channels at $200 \mathrm{~Hz}$. The sensors were spaced evenly in the radial direction at 4 meter spacing, and each array arm was at 15 degree increments around an arc (see Fig. 3.33).

Large Aperture Array A large aperture array of 49 wireless NCPA-UMX pressure sensors was distributed on the ground covering a 400-meter aperture. The center of the large aperture array did not coincide with that of the small aperture array. Data was acquired at $1000 \mathrm{~Hz}$ and each sensor was GPS time synchronized. The array included 6 arms spaced at 60 degree increments. Along each arm the sensors were distributed with spacing that increased as powers of 2 with smaller spacing near the array center (see Fig. 3.34). The layout was the same as in Fig. 3.20. 


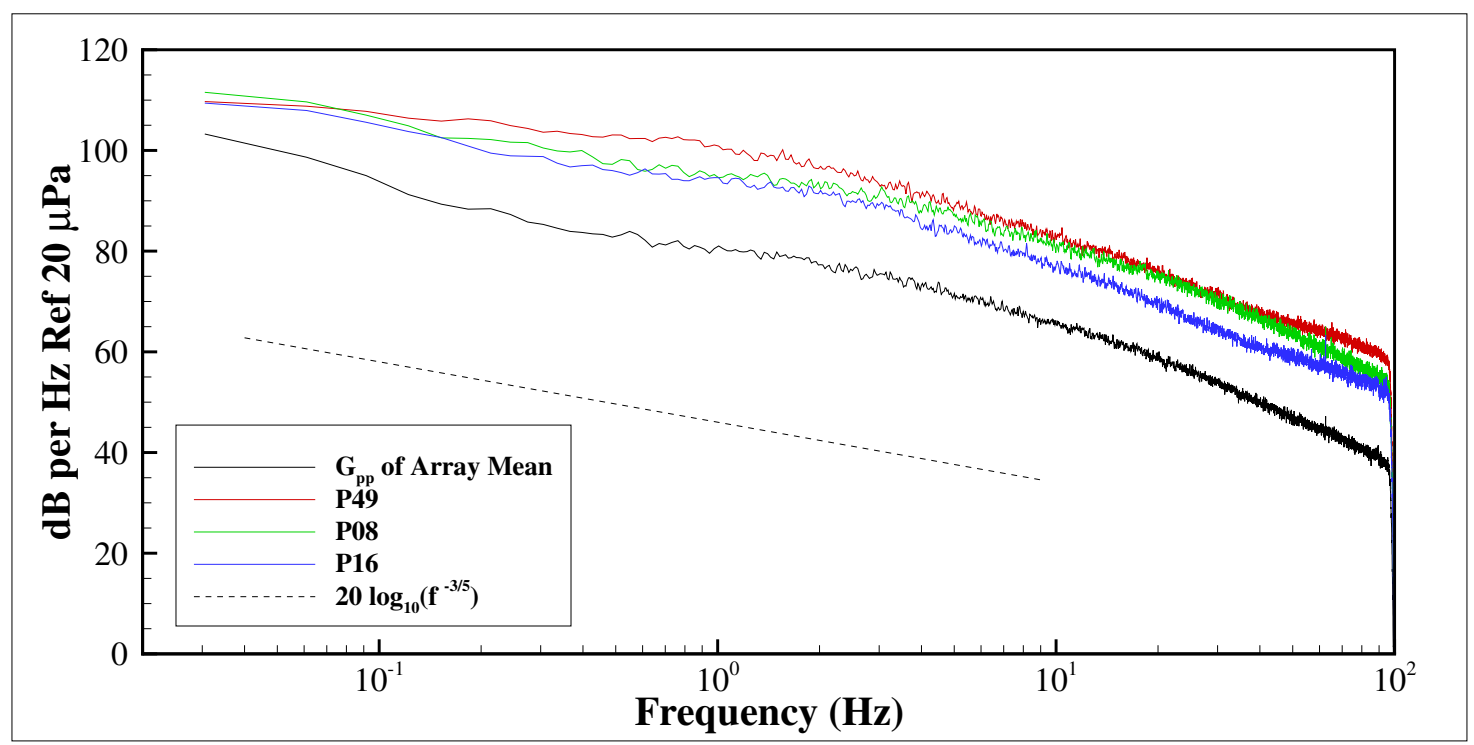

Fig. 3.30. One-sided auto-spectral density as in Fig. 3.29 but for the Tuesday noon data.

ASC SODAR An ASC-3000S SODAR system was setup just to the west of the meteorological tower to acquire data for analysis using the pulse-to-pulse method developed under this program (see Fig. 3.35). Analysis of this data is discussed in Chapter 2, Section 2.6.

Each of the measurement systems was operated continuously for 44 hours beginning at 12:00 CST on 21 June 2011. Fig. 3.36 plots the average of all 96 sensors in the small aperture array showing the higher pressure fluctuations characteristic of daytime conditions over the duration of the experiment.

Figures 3.37 through 3.42 show the general characteristics of the flow environment observed from the 200-meter tower at the Reese Technology Center field site. Wind speeds were measured with both sonic anemometers and propellor-type anemometers, and the two measurements agree (Figures 3.37 and 3.38). Fig. 3.39 shows the wind direction which was predominantly out of the North initially and shifted to be from the West/Southwest for much of the last half of the experiment. The quick changes in wind speed early in the evening on Tuesday correspond to very low wind velocities, so the $\tan ^{-1}$ was very sensitive to small gusts in the wind. Fig. 3.40 shows the vertical wind speed which picked up and was a significant 1.5-2.0 m/s above 100 meters from Thursday onward. Fig. 3.41 shows the temperature profile which exhibits the typical diurnal cycle with the high temperature being at its peak Wednesday evening. Finally, Fig. 3.42 shows the pressure spectra from the MRT pressure array. These are all presented here to document the atmospheric conditions during the experiment.

The RTC deployment was intended to provide measurements at a site where a wind turbine was in active operation. The intent was to gather atmospheric data coincident with acoustic data to observe the noise produced by the active turbine under varying atmospheric conditions. However, the research turbine at the Reese Technology Center was not under the direct control of the Wind Science and Engineering researchers at the time of the experiment. 


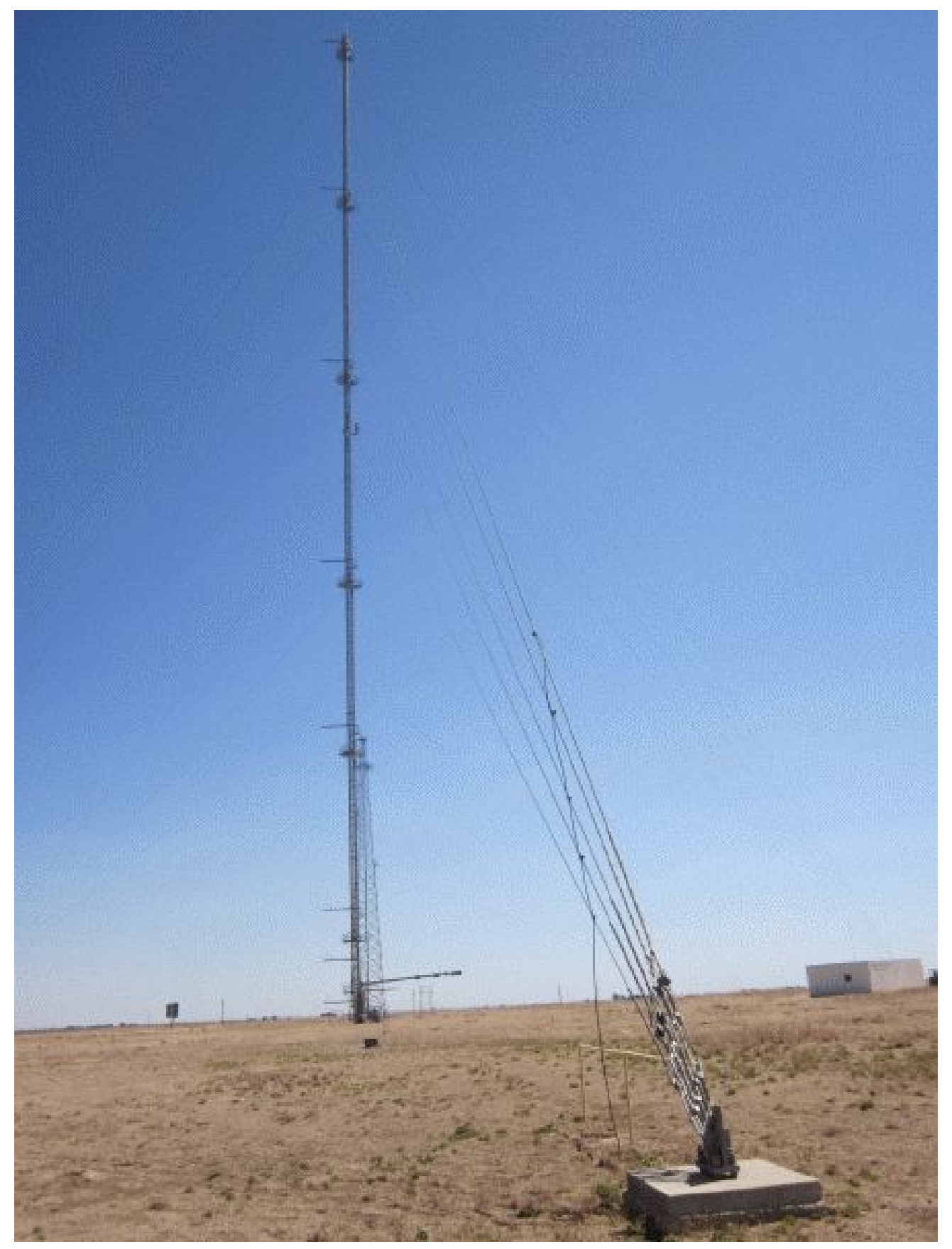

Fig. 3.31. The photo shows the entire 200 meter height of the meteorological tower at the Reese Technology Center. The tower includes measurements at 10 stations along its vertical extent. 


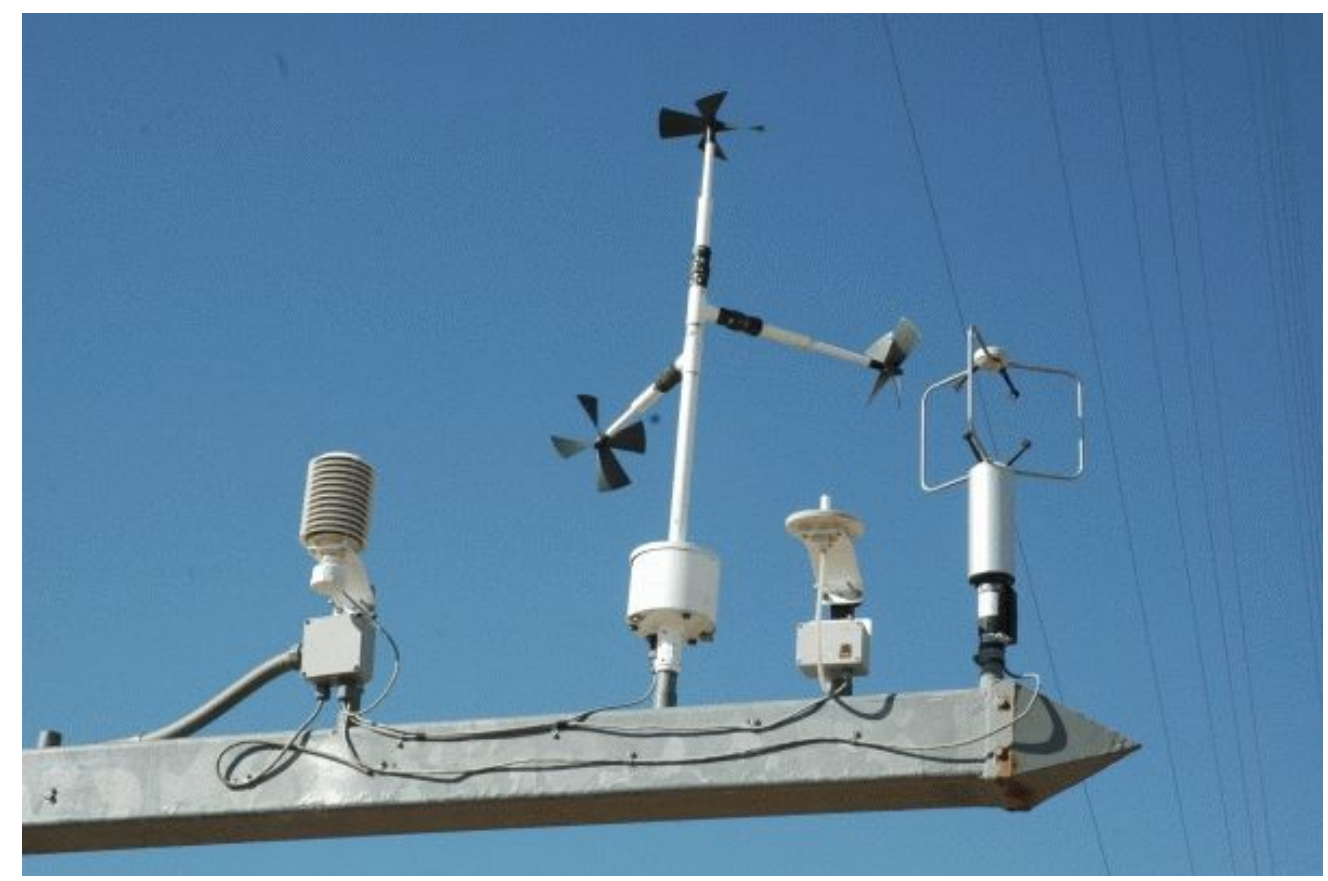

Fig. 3.32. The meteorological tower at the Reese Technology Center includes measurements at 10 stations. This photo shows the equipment at each station which include a sonic anemometer, propeller anemometer, temperature sensor, and humidity sensor. Three of the stations include a barometric pressure measurement.

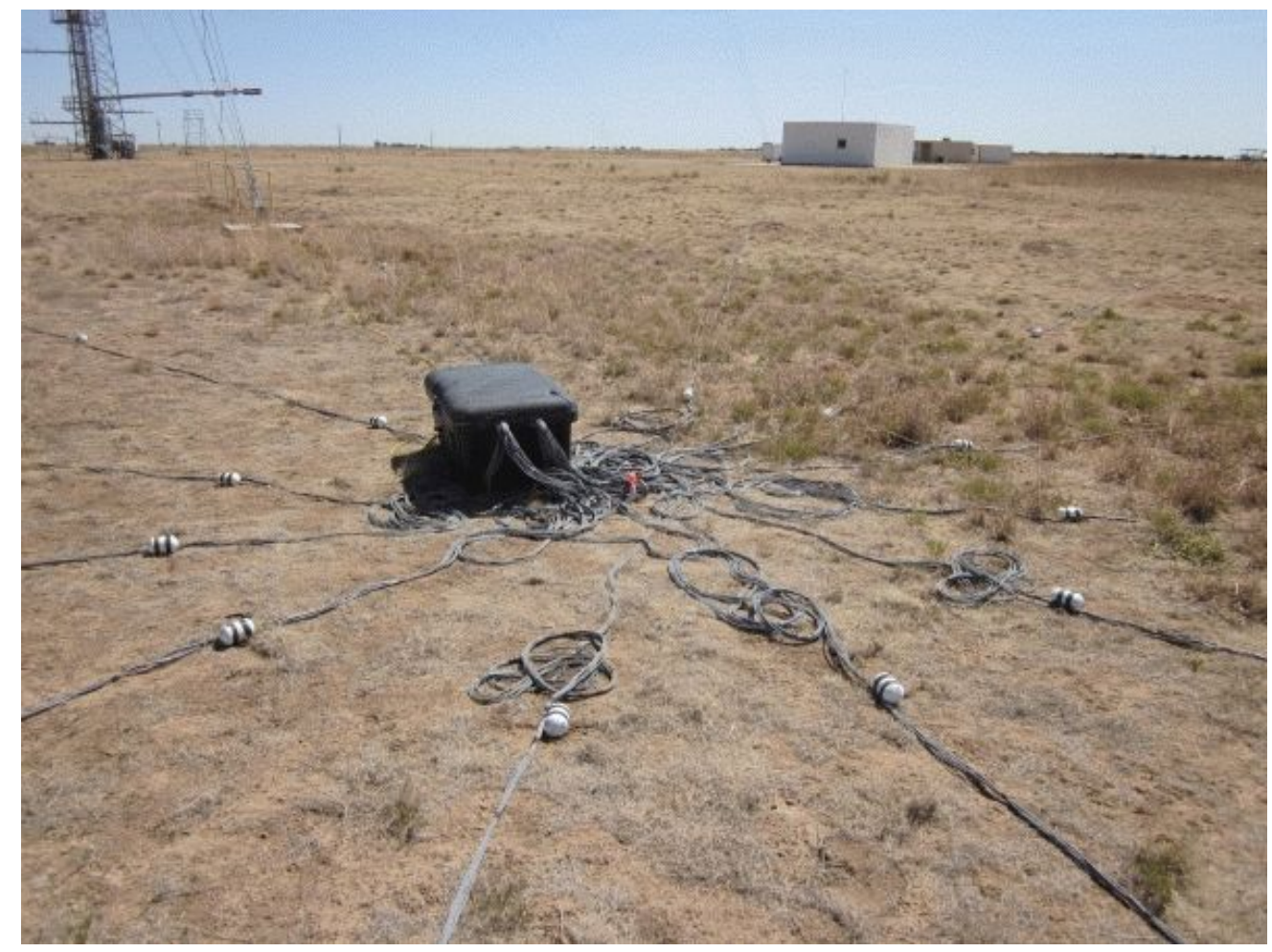

Fig. 3.33. Small aperture array setup for the Lubbock, TX, experiment. 


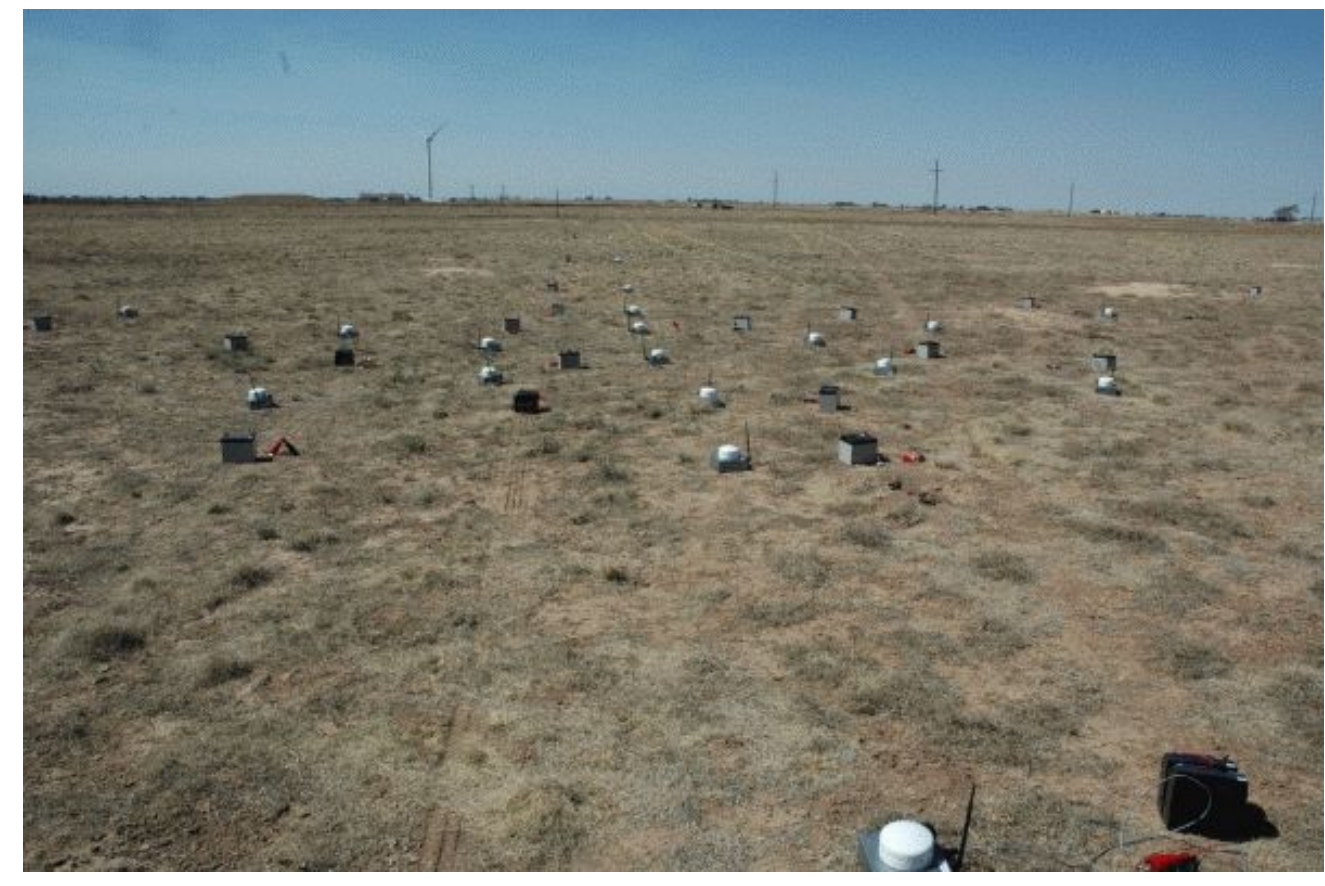

Fig. 3.34. Large aperture array setup for the Lubbock, TX, experiment.

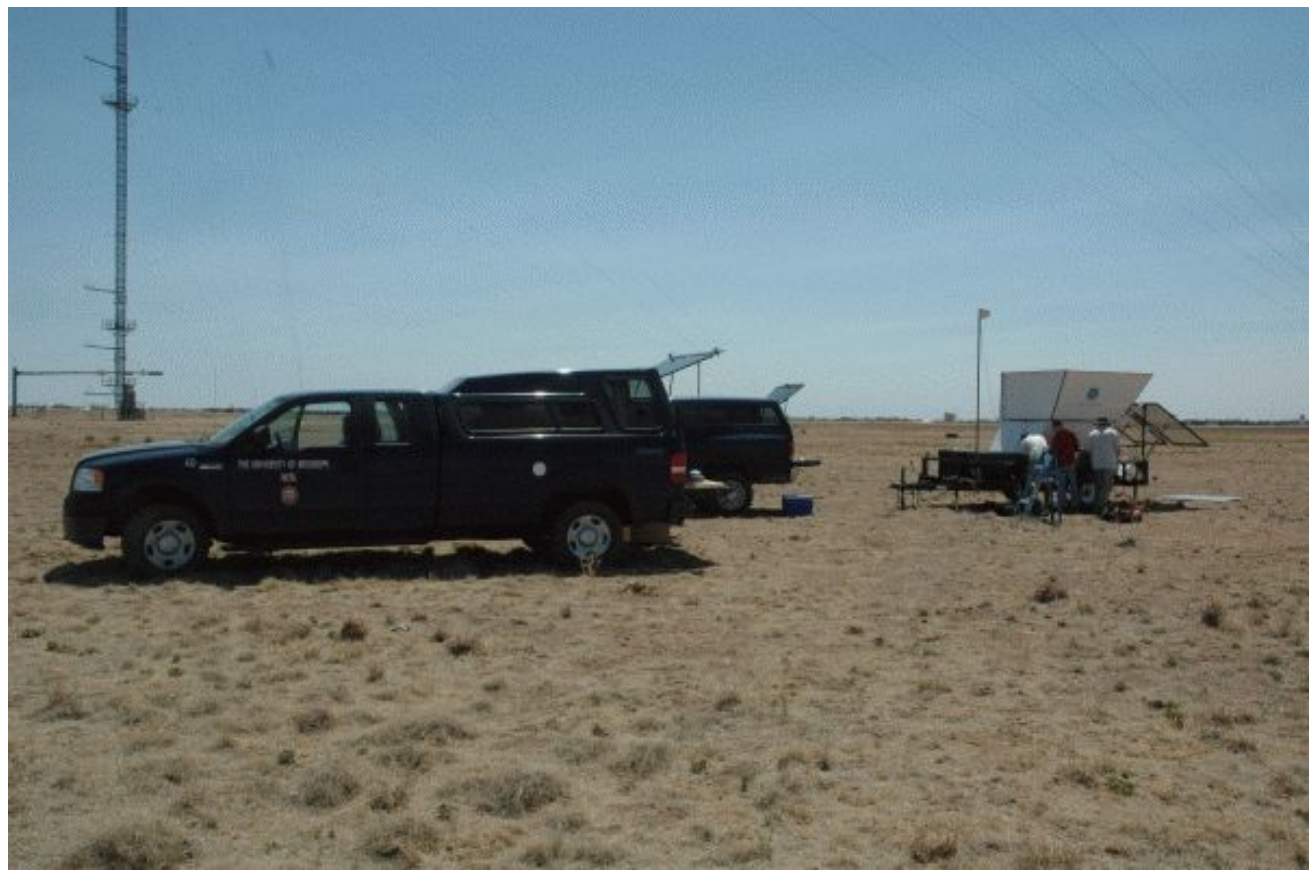

Fig. 3.35. The ASC SODAR system was positioned just West of the meteorological tower (in the left background). 


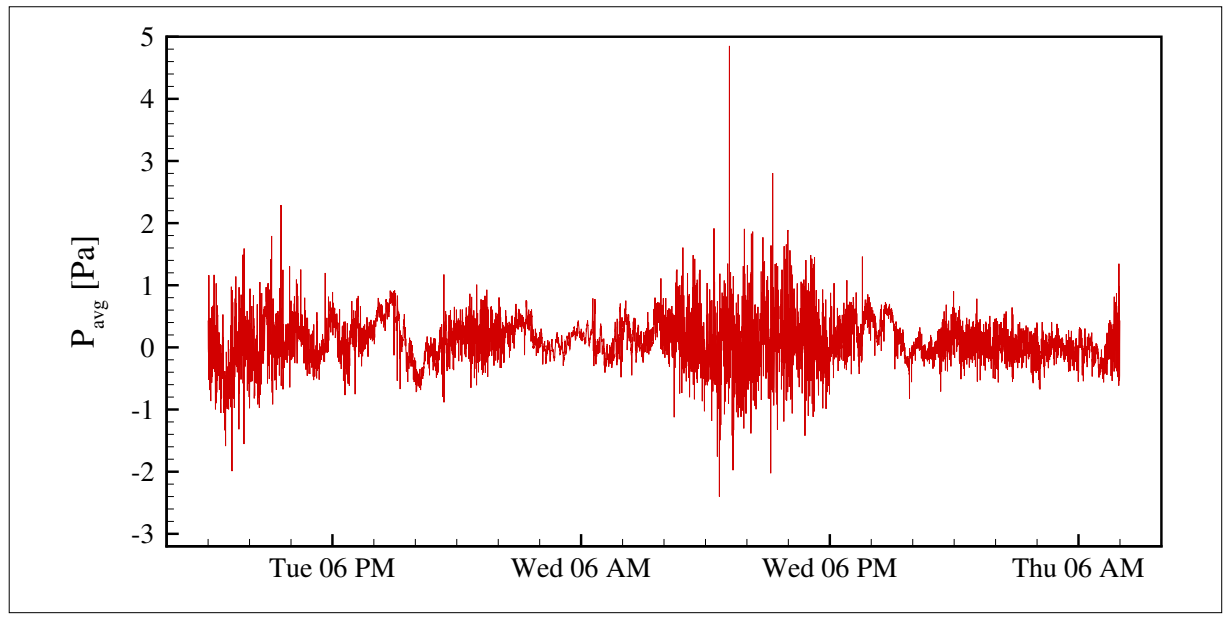

Fig. 3.36. The spatial average of all 96 sensors in the small aperture array plotted versus time over the duration of the Lubbock experiment.

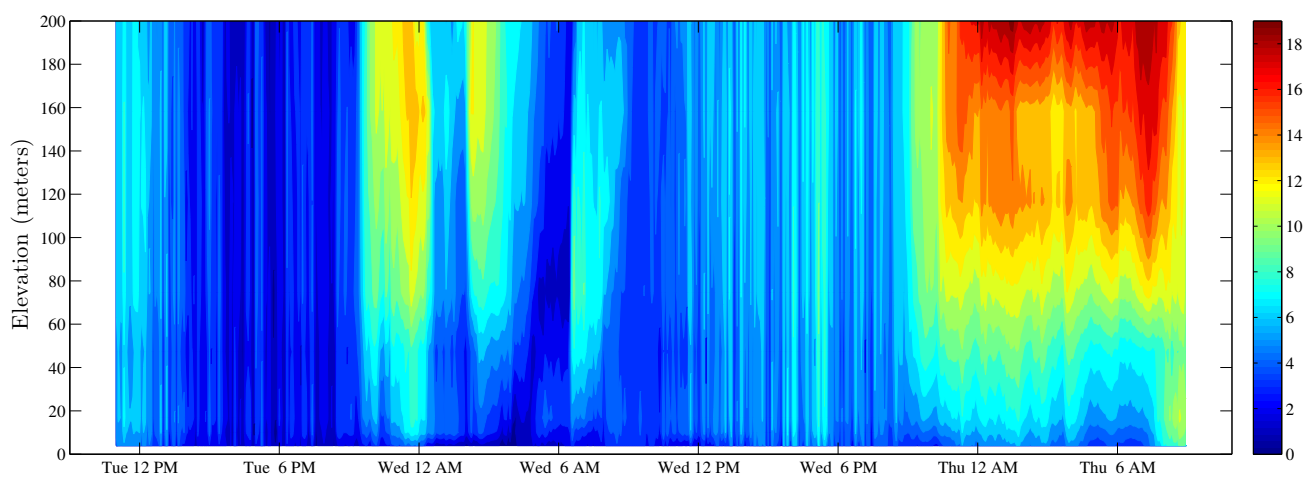

Fig. 3.37. Wind speed $\left(\sqrt{U^{2}+V^{2}}\right)$ from the Prop-Anemometers. Units are $\mathrm{m} / \mathrm{s}$.

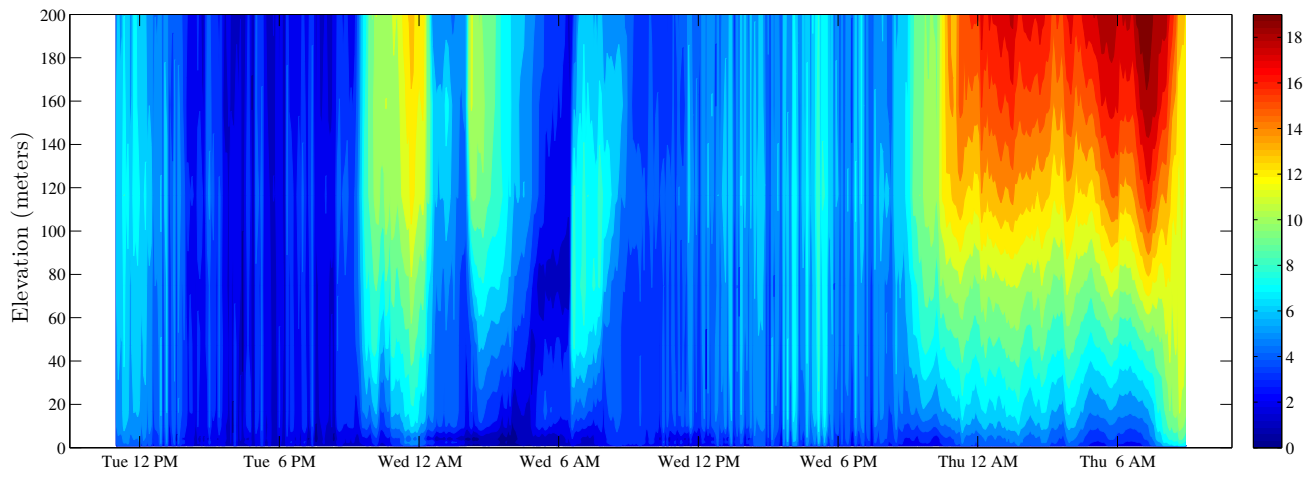

Fig. 3.38. Wind speed $\left(\sqrt{U^{2}+V^{2}}\right)$ from the Sonic Anemometers. Units are $\mathrm{m} / \mathrm{s}$. 


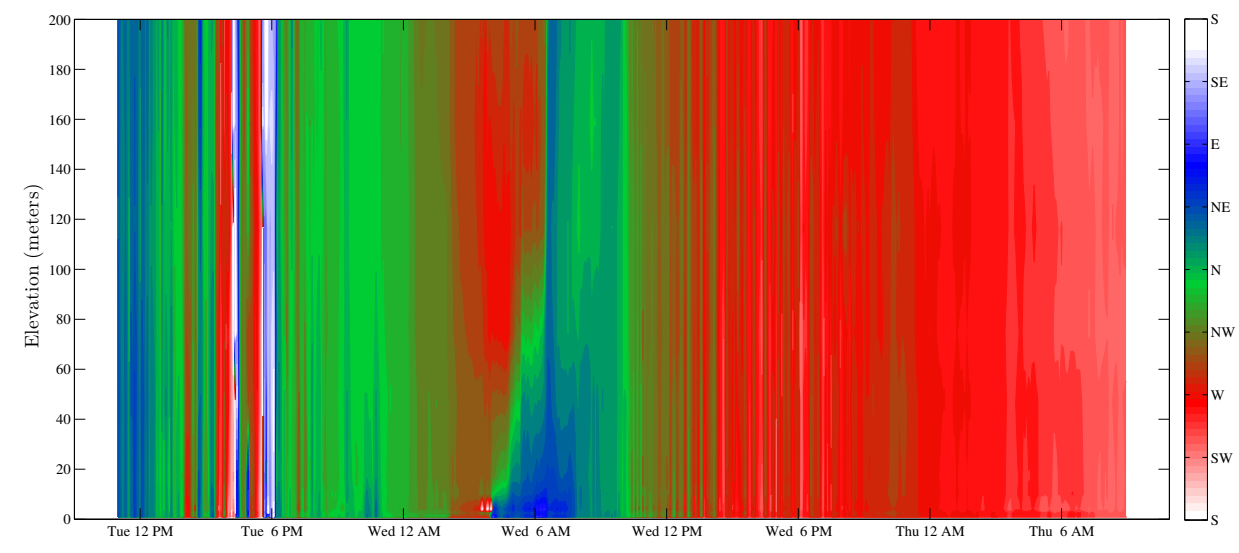

Fig. 3.39. Wind direction $\left(\tan ^{-1}(-V / U)\right)$ determined from the Sonic Anemometer horizontal velocity components.

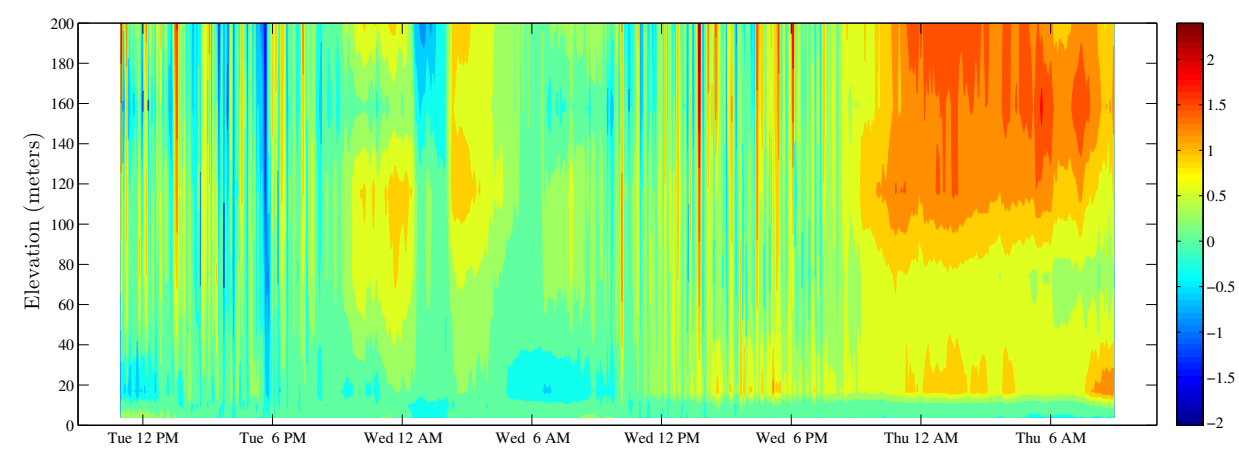

Fig. 3.40. Vertical wind speed measured by the Prop-Anemometers. Units are m/s.

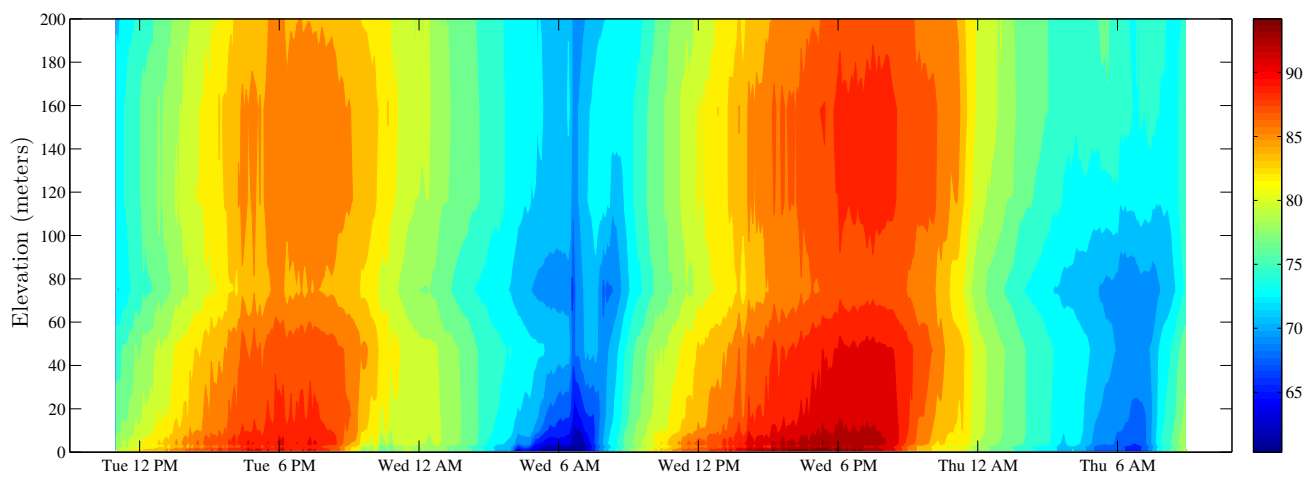

Fig. 3.41. Atmospheric temperature. Units are ${ }^{\circ} \mathbf{F}$. 


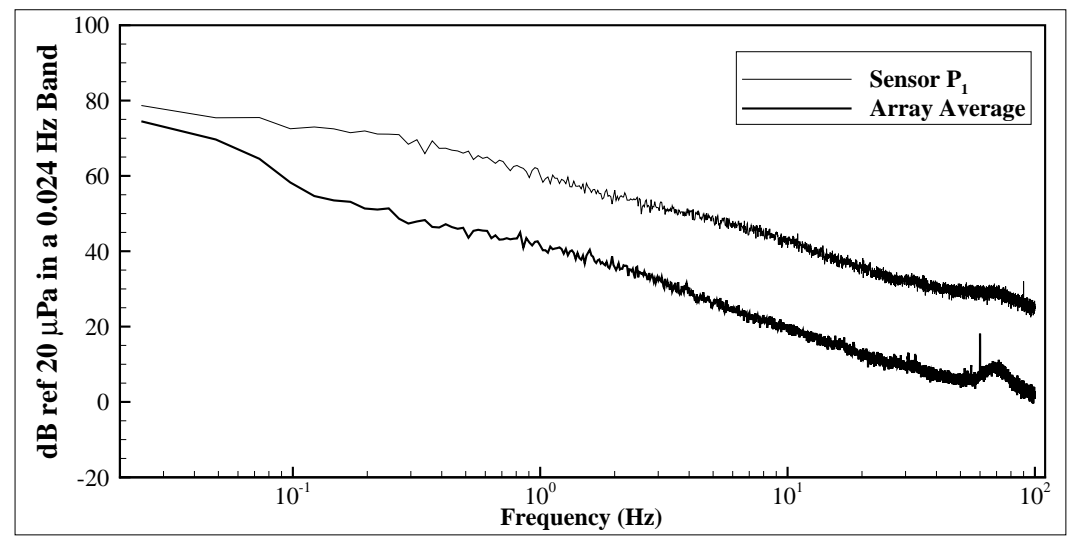

Fig. 3.42. Auto-spectrum of the surface pressure fluctuations averaged over a $\sim 22$ minute period starting from 23:00:00, Tuesday, 21 June 2011. ( $8192 \times 32$ samples at $200 \mathrm{~Hz}$.)

Rather, the manufacturer, Alstom Power, was in the process of assessing a modified blade design. As a result, the turbine was not operational during the field experiment. Therefore, it remains a goal of future work to obtain acoustic data over the infra-sound and audible ranges to compare with the results obtained under the computational efforts of this program (see Chapter 5).

\subsection{Velocity Profile Estimation}

Section 3.3.2 established the fundamental basis for pressure/velocity coupling in the ABL and demonstrated that horizontal gradients in the surface pressure fluctuations are dependent on the vertical gradients in the velocity. The ability to utilize this framework to determine convection velocities was established by Shields' analysis of the Piñon Flat data presented in Section 3.4. To move these concepts toward a deployable system, it was necessary to achieve an automated methodology for data analysis that would demonstrate estimation of the entire velocity profile. This section presents the development of just such an analysis method and shows the successful application to both the wind tunnel data described in Section 3.5.1 and the RTC data described in Section 3.5.2.2.

\subsubsection{Establishing the Link Between Pressure and Velocity Length Scales}

The cross-correlation functions, $\rho_{p_{i}^{\prime}, p_{j}^{\prime}}$, in (3.6) provided a means to compute a convection velocity and an integral time scale for the surface pressure signals. This analysis was performed on the wind tunnel data described in Section 3.5.1. The convection velocity, $U_{c}$ was determined by selecting the time lag, $\tau$, for the maximum cross-correlation. This $\tau_{\max }$ varied depending on the spatial separation, $\xi$, between the sensors in question (as shown in Fig. 3.18). Fig. 3.43 shows the obtained convection velocity using this method normalized by the related free-stream velocity. Compared to the boundary layer profile in Fig. 3.14, $U_{c}$ appears to follow the same trend if the microphone separation is taken as an analogy for the vertical height in the boundary layer. Also, it appears that the heat addition at the boundary slightly increased the convection velocity. 


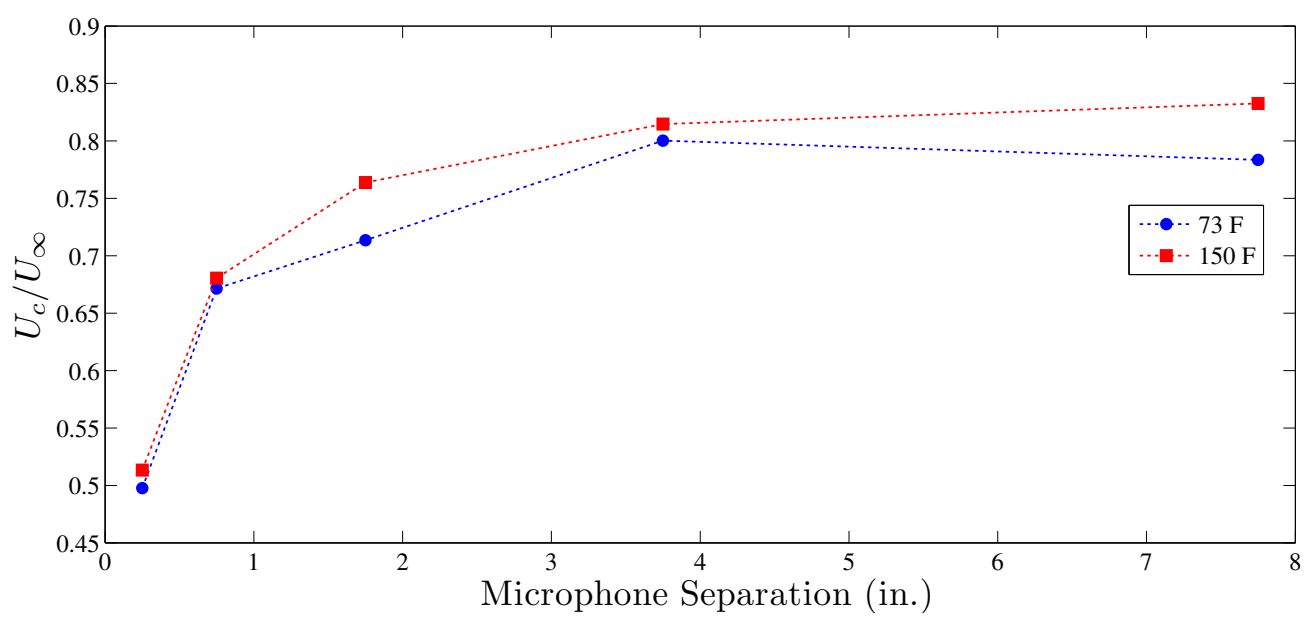

Fig. 3.43. Convection velocity computed from the cross-correlation between microphone 1 and microphones 2 through 6.

In order to convert the sensor spacing to an elevation above the measurement surface, it seemed appropriate to first look at the time scale. The estimate of the integral time scale was obtained from the auto-correlation of the pressure signals by integration:

$$
\theta=\int_{0}^{\infty} \rho_{p_{1}, p_{2}}(0, \tau) d \tau
$$

The time scale was then normalized by the free-stream velocity, $U_{\infty}$, and the boundary layer displacement thickness, $\delta^{\star}$ :

$$
\theta^{\star}=\theta U_{\infty} / \delta^{\star}
$$

Fig. 3.44 shows that the time scale is approximately 1.1 for the heated surface and 1.6 for the unheated surface. It is expected that the heat addition at the boundary, which causes additional buoyant convection, would result in a more rapid breakdown of the turbulent structures. Therefore, this result follows expectations and suggests that the time scale would continue to decrease for increased surface temperature.

Finally, the convection velocity and integral time scale were combined to yield a estimate for the longitudinal (streamwise) length scale, $L_{x}$ based on the surface pressure signals:

$$
L_{x}=U_{c} \theta \text {. }
$$

Fig. 3.45 shows that the heat addition at the boundary resulted in a decrease in the integral length scale. Given that increasing $T_{s}$ also corresponded to a decrease in the time scale, $\theta^{\star}$, and a slight increase in $U_{c}$, it appears that the time scale is more sensitive to surface temperature than convection speed. As a result, the length scale decreases but not as fast as the time scale decreases.

These initial results were significant because they point to the ability to estimate the flow velocity in the boundary layer from the surface pressure convection speed by considering the length and time scales present in the boundary layer. The relative insensitivity of the convection speed with surface temperature was promising because it suggests that an established velocity estimation method would not depend on surface temperature. Further research is needed to establish this independence. 


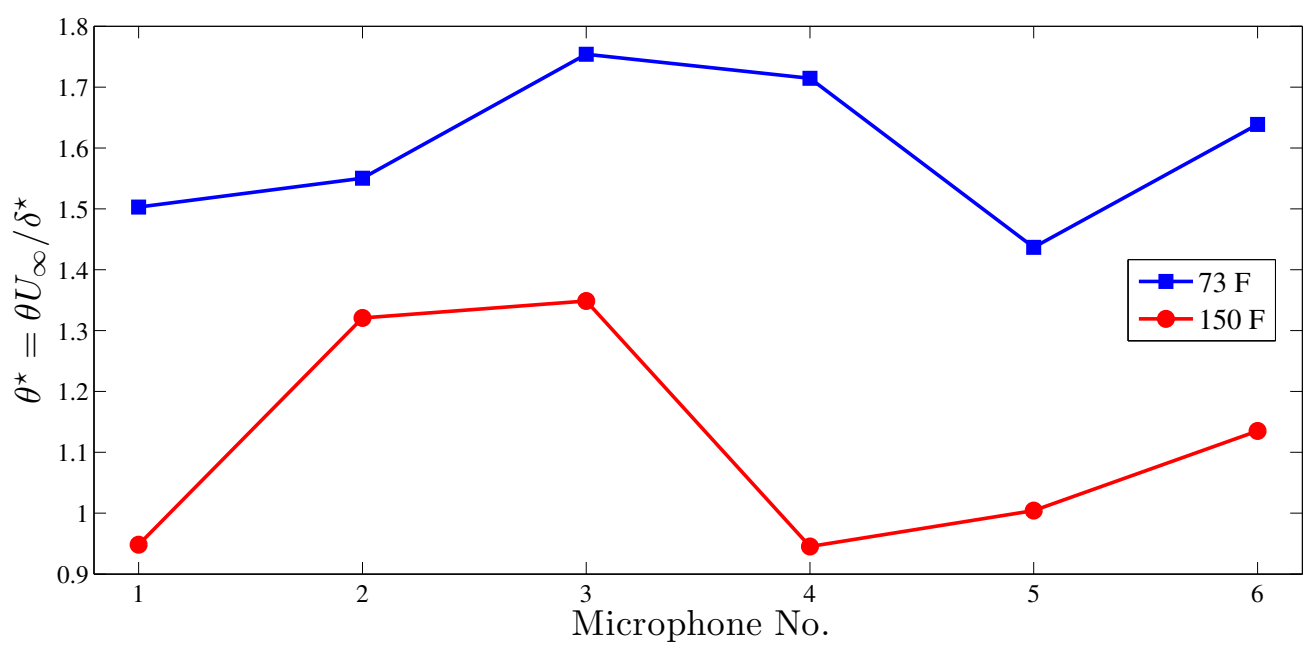

Fig. 3.44. Normalized integral time scale observed from the surface pressure signals for the two surface temperature conditions.

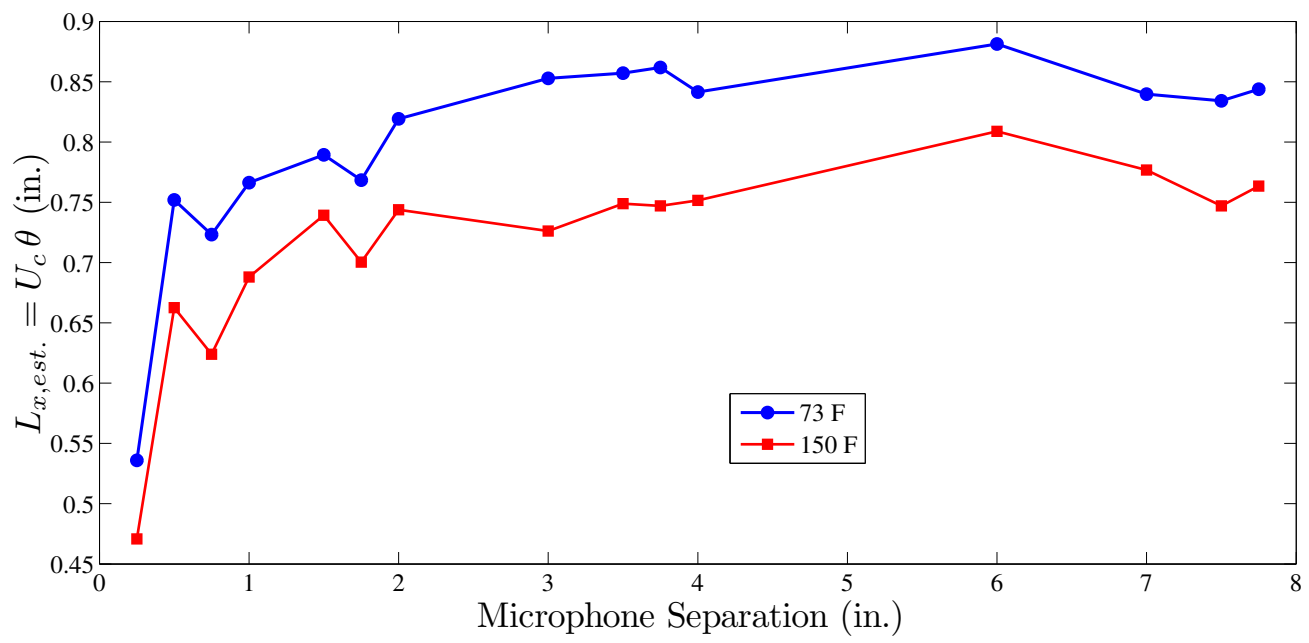

Fig. 3.45. Estimated length scale based on the surface pressure data for the two surface temperature conditions. 


\subsubsection{Velocity Profile Estimation Methodology}

In order to automate the Shields analysis method and at the same time take the characteristic time scales into account, an analysis method based on the coherence was adopted. The first step of the analysis was to calculate the magnitude squared coherence at each frequency between the signals of two sensors. The coherence function, $C_{s_{1} s_{2}}(f)$, was calculated from the power spectral densities of each signal $\left(P_{s_{1} s_{1}}(f)\right.$ and $\left.P_{s_{2} s_{2}}(f)\right)$ and the cross power spectral density $\left(P_{s_{1} s_{2}}(f)\right)$ of both of them:

$$
C_{s_{1} s_{2}}(f)=\frac{\left|P_{s_{1} s_{2}}(f)\right|^{2}}{P_{s_{1} s_{1}}(f) P_{s_{2} s_{2}}(f)} .
$$

Based on the coherence results a frequency range of interest was defined. The beginning of the range was defined as a frequency where coherence greater than $5 \%$ was recorded for four consecutive frequencies. Similarly, the end of the range was defined as the frequency where four consecutive coherence values were below the set value. Requiring 4 consecutive values above/below the 0.05 limit was chosen based on experience with the data and served to provide some filter for the frequency range selection.

In the select frequency range, the complex transfer function, $T_{s_{1} s_{2}}(f)$, was computed as the quotient of the cross power spectral density of the two signals and the power spectral density of the first signal:

$$
T_{s_{1} s_{2}}(f)=\frac{P_{s_{2} s_{1}}(f)}{P_{s_{1} s_{1}}(f)} .
$$

From this, the lag time, $\tau(f)$, was calculated at each frequency by unwrapping the phase angle of the transfer function and dividing it by frequency.

Next, following the method of Priestly ${ }^{[18,19]}$ the known separation distance between the microphones, $d$, was divided by the time lag in order to calculate the convection velocity, $U_{c}(f)=d / \tau(f)$, at the select frequencies. Knowing the the boundary layer must merge with the freestream, the wave-length, $\lambda$ was estimated by dividing the freestream velocity by the frequencies in the selected frequency range: $\lambda(f)=U_{\infty} / f$. The two variables, when plotted against each other resulted in a relationship $\lambda\left(U_{c}\right)$. Assuming an exponential curve consistent with boundary layer theory, best-fit equation was applied to determine the constants $A_{c}$ and $B_{c}$ such that

$$
\lambda\left(U_{c}\right)=A_{c} e^{B_{c} U_{c}} .
$$

The next step is to determine the appropriate relationship between the length scale $\lambda$ and the corresponding altitude, $H$, allowing the estimation of the vertical velocity profile. To do this, known velocity data (from either the low-speed tunnel hot-wire or the RTC meteorological tower) was fit with an exponential function such that

$$
H=A_{e} e^{U \times B_{e}},
$$

where $H$ is the altitude and $U$ is the velocity. Then, by requiring the two exponential curves (3.12) and (3.13) to match, the constants can be related by

$$
A_{e}=A_{c} \times \frac{d}{C_{a}}, \text { and }
$$




$$
B_{e}=B_{c} \times\left|U_{c, \min }-U_{c, \max }\right| \times C_{b} .
$$

The goal was the to compare all the collected data to establish $C_{a}$ and $C_{b}$ as constants which could be used for subsequent estimation of the velocity profile. For the low-speed tunnel data, it was found that $C_{a}=60$ and $C_{b}=1.65$ could be used as constants to successfully analyze the entire data set. However, in analyzing the RTC met. tower data, the best fit required $C_{a}=0.57$ and $C_{b}=13$ which was significantly different from the wind tunnel data. Nevertheless, for the tunnel data, once these constants were known, the pressure data could be used to estimate the velocity profile with great accuracy. Results demonstrating this are presented next.

\subsubsection{Velocity Profile Estimation Results and Discussion}

The velocity estimation methodology was applied first to the wind tunnel data and later to the RTC data. In the case of the wind tunnel data, three runs were considered with nearly identical freestream speed of $7.9 \mathrm{~m} / \mathrm{s}$ but with three different surface temperatures: 23,32 , and $50^{\circ} \mathrm{C}$. The streamwise velocity profile was estimated from the surface pressure coherence. In the case of the RTC field tests, a 30 minute long segment of the data starting at 04:00 UTC on 22 June 2012 was considered for a given array arm pair of sensors. The velocity profiles in $\mathrm{u}, \mathrm{v}$, and $\mathrm{w}$ directions were estimated.

\subsubsection{Analysis of Wind Tunnel Data}

To fully demonstrate the procedure, the analysis of the room temperature data $\left(23^{\circ} \mathrm{C}\right)$ is presented step-by-step. The coherence measurements were constructed from the signals measured by microphones 3 and 4. In Fig. 3.46, the coherence between the signals is plotted with the frequency range having consistent coherence above $5 \%$ highlighted as noted in the discussion following Equation 3.10. For reference, the individual spectra of both signals is shown in Fig. 3.47 with the same frequency range highlighted. The selected frequency range in this case was found to be between approximately $2400 \mathrm{~Hz}$ and $5000 \mathrm{~Hz}$.

Fig. 3.48 presents both the real and imaginary parts of the calculated transfer function between the two signals along with the highlighted frequency range. Fig. 3.49 presents the time lag, $\tau(f)$, between the two microphones calculated from the transfer function as suggested by Equation (3.11).

The calculated time lag and select frequency values, as explained in the previous section, were used to calculate the convection velocity and wavelength estimates. The two variables plotted against each other are shown in Fig. 3.50 along with a best-fit exponential line with $A_{c}=0.0057$ and $B_{c}=0.0191$. The crucial next step of the procedure considered the mean, hot-wire acquired, streamwise, velocity profile. The constants of Equation 3.13 were calculated following Equations 3.14 and 3.15, and the resulting exponential curve estimating the velocity profile was plotted along with the measured mean velocity profile, as shown in Fig. 3.51.

The same procedure was repeated for tests at 32 and $50^{\circ} \mathrm{C}$. The resulting estimated velocity profile along with the measured mean velocity profile are illustrated in Fig. 3.52. Over the range of conditions encountered in the wind tunnel experiment, the estimating equation was found to be independent of the surface temperature in the considered 


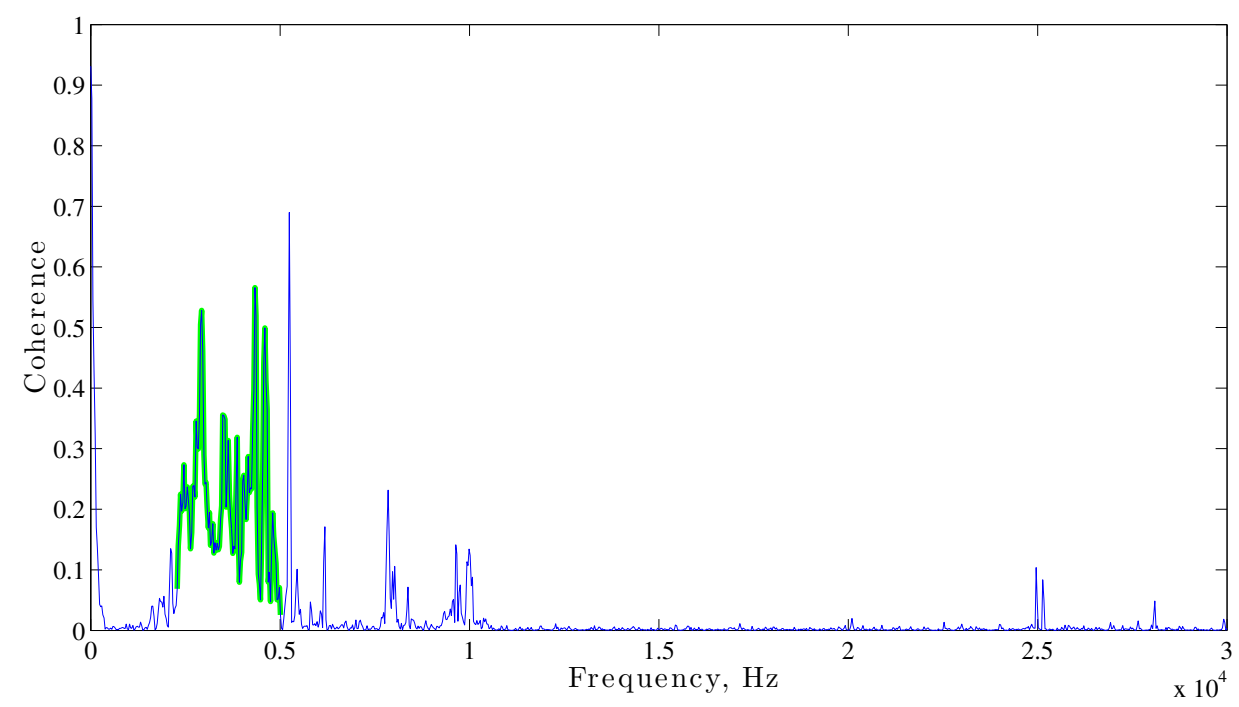

Fig. 3.46. The coherence between signals of pressure sensors 3 and 4 for wind tunnel tests at room temperature; highlighted is the select frequency range of interest.
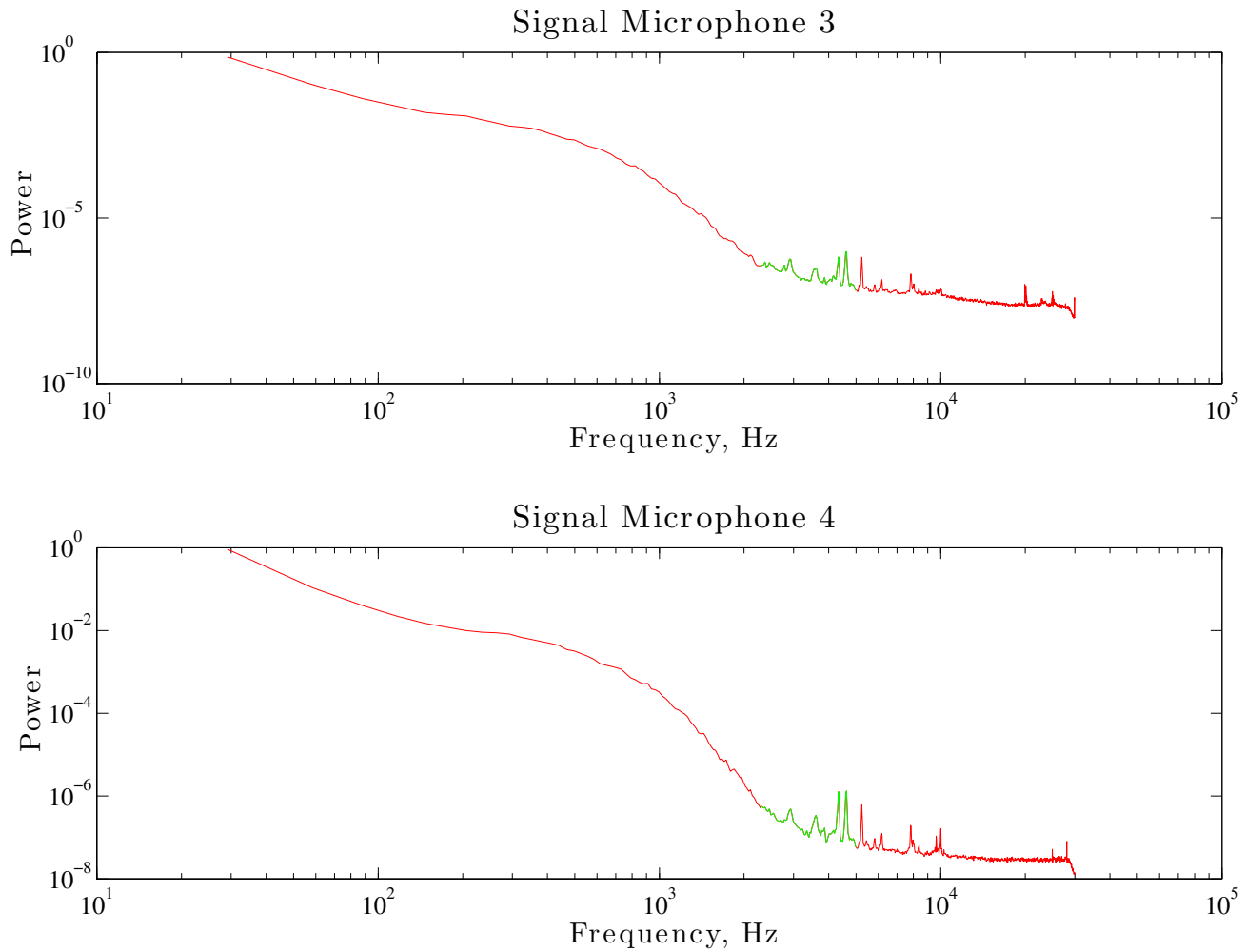

Fig. 3.47. The spectra of pressure measurements acquired using sensors 3 and 4 for wind tunnel tests at room temperature; highlighted is the select frequency range of interest. 


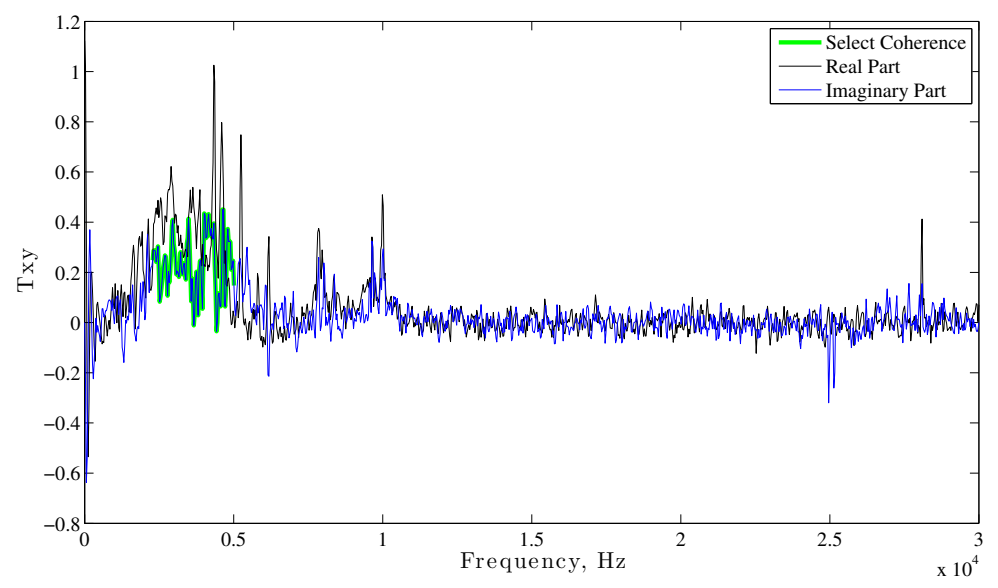

Fig. 3.48. The real and imaginary parts of the transfer function between the two signals; highlighted is the select frequency range of interest.

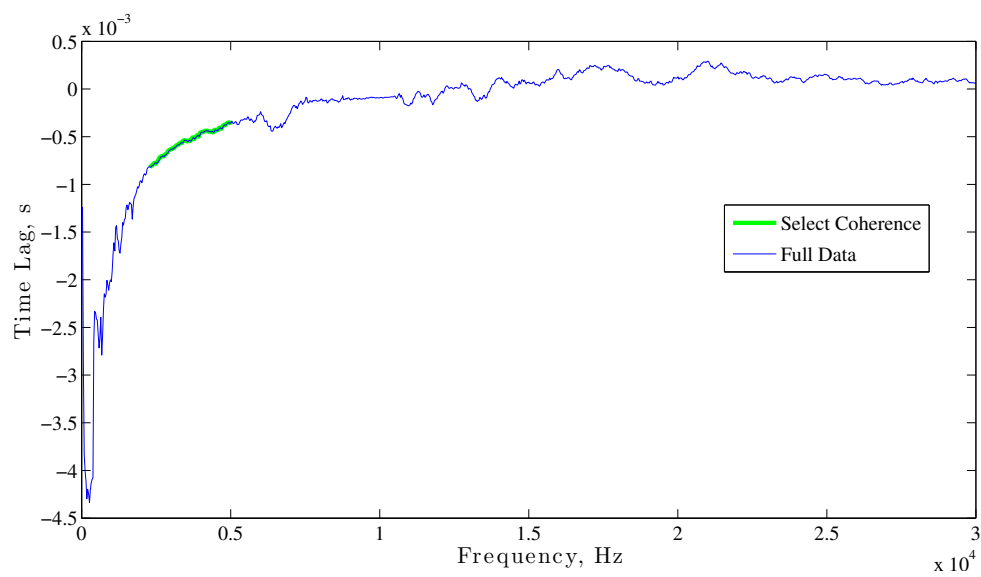

Fig. 3.49. The time lag between the two signals at various frequencies; highlighted is the select frequency range of interest.

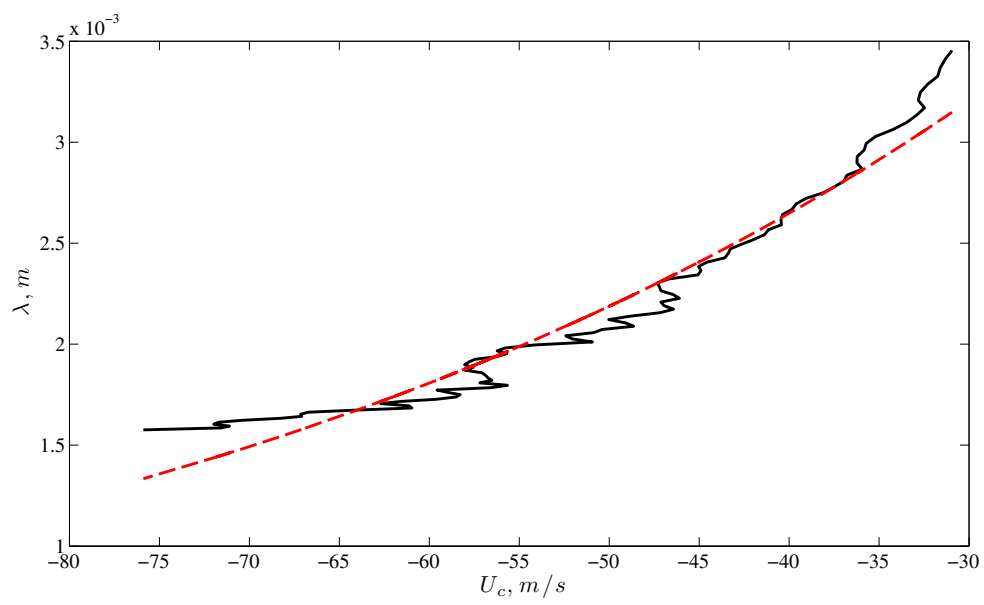

Fig. 3.50. Plot of calculated convection velocity and wavelength at the select frequency range along with a best-fit exponential curve. 


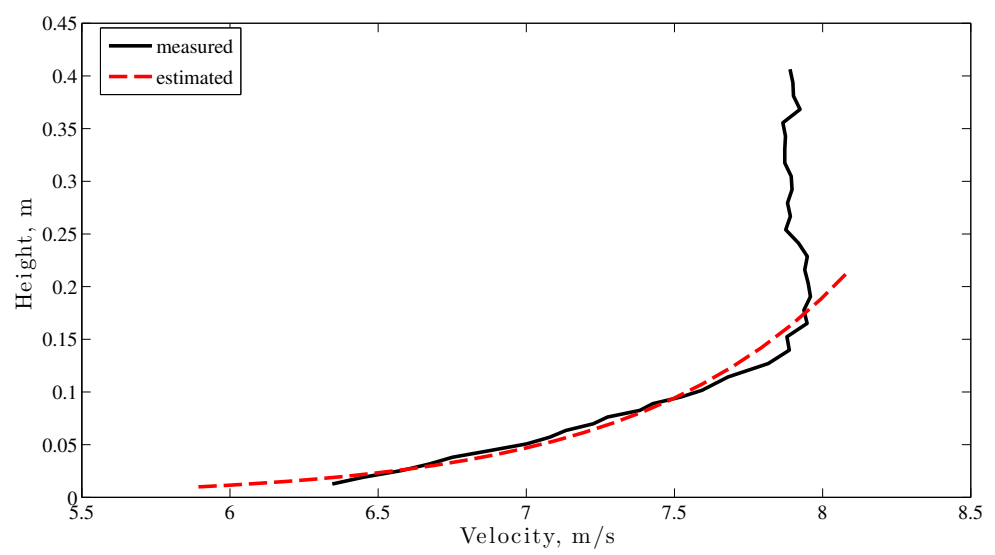

Fig. 3.51. Plot of the hot-wire measured velocity profile along with the velocity profile estimated from two ground pressure sensors for wind tunnel tests at $23^{\circ} \mathrm{C}$.

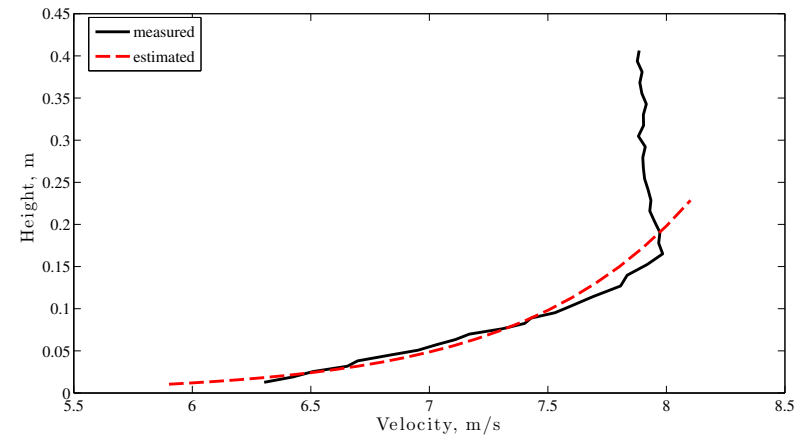

(a) $32{ }^{\circ} \mathrm{C}$

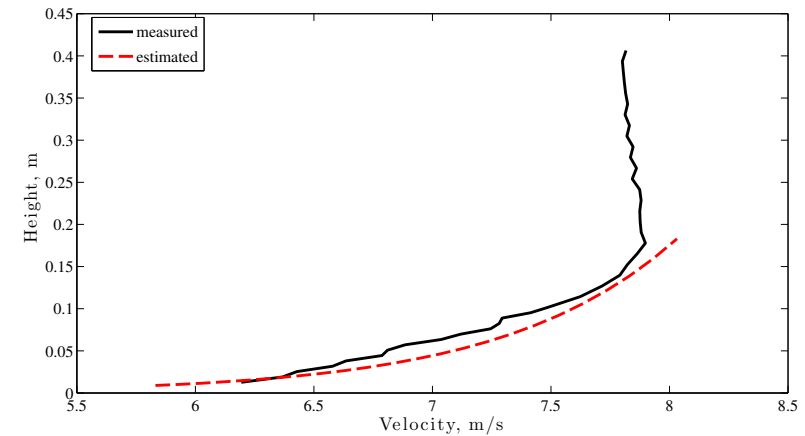

(b) $50^{\circ} \mathrm{C}$

Fig. 3.52. Comparison of hot-wire measured velocity profiles along with the velocity profiles estimated from two ground pressure sensors for wind tunnel tests at 32 and $50^{\circ} \mathbf{C}$.

range. Therefore, the values of the constants mentioned above were not altered to produce these estimates.

\subsubsection{Analysis of RTC Field Data}

The analysis of the field data was performed for only one single 30 minute segment of data starting at 04:00 UTC, 22 June 2012. This was a test to determine the applicability of the methodology developed in the wind tunnel test. The methodology was only applied to two of the 96 sensors in the MRT array. It was immediately apparent that the analysis would be more difficult based on the much lower levels of coherence, plotted in Fig. 3.53, compared to the wind tunnel coherence plot of Fig. 3.46. Above $0.1 \mathrm{~Hz}$, the coherence is never continuously above $5 \%$, so no automated way of selecting a frequency range based on coherence was determined. Instead, in this case, a frequency range was selected from the time lag data, where a "hump" was noted from its lowest to its highest point (smallest and largest time lag value). The calculated time lag along the whole frequency range is shown in Fig. 3.54, and the selected range is highlighted. 


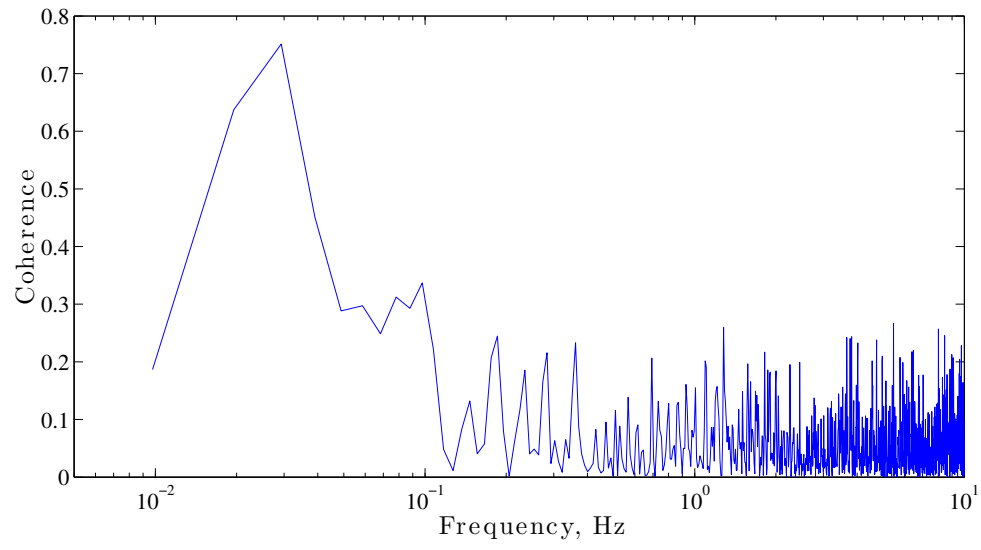

Fig. 3.53. The coherence between signals of pressure sensors 1 and 2 of L1 arm of the sensor array pointing north in the field tests.

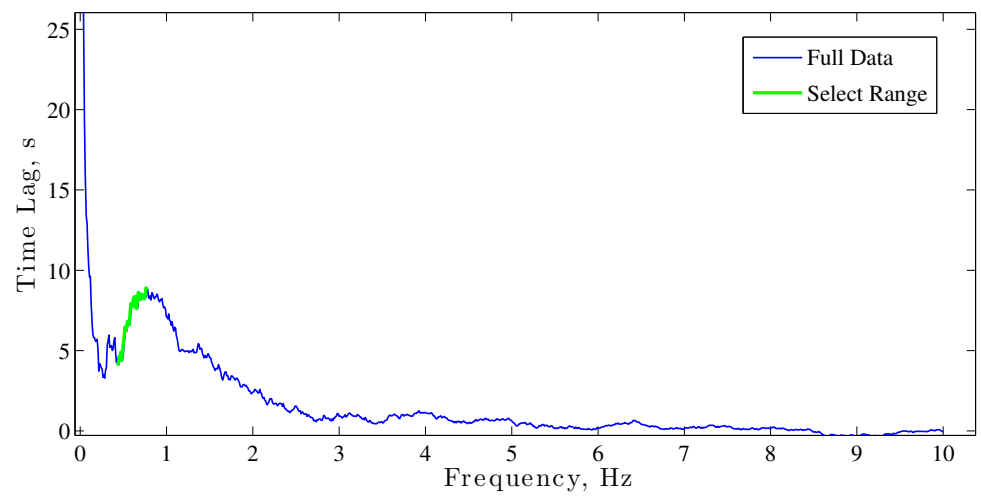

Fig. 3.54. The time lag between the two signals at various frequencies; highlighted is the select frequency range of interest. 


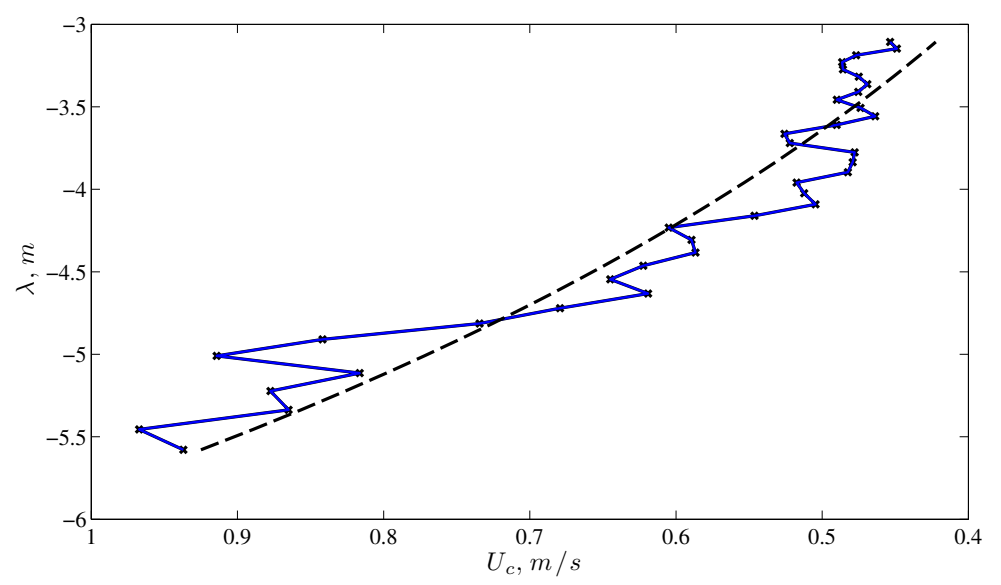

Fig. 3.55. Plot of calculated convection velocity and wavelength at the select frequency range along with a best-fit exponential curve.

Following the methodology from the wind tunnel data, the convection velocity and wavelength were calculated and best-fit exponential equation was fitted, as shown in Fig. 3.55, to determine the $A_{c}$ and $B_{c}$ constants. Next, the constants of Equation 3.13 were calculated following Equations 3.14 and 3.15 and the resulting exponential curve estimating the velocity profile was plotted along with the measured mean velocity profile. Curiously, it was found that the same equation and constants were applicable independent of which velocity direction $(u, v$, or $w$ ) was considered (although it should be noted that the procedure used the corresponding component of freestream velocity which was known from the tower data). The comparison of meteorology tower measured velocity profiles along with those estimated from two ground pressure sensors is shown in Fig. 3.56 for all three velocity directions.

At the conclusion of the program it was noted that the selection of the frequency range from the time lag "hump" was erroneous. This selection happened to correspond to increasing time lag magnitude with increasing frequency (decreasing wavenumber) which is opposite of that in the wind tunnel data. This is also opposite of what is expected for boundary layers where the larger scales should move faster at farther distances from the wall. Another look at Fig. 3.54 shows that the overall trend is for time lag decreases with frequency over the range from 0 to $3 \mathrm{~Hz}$. The increase in time lag around $0.6-0.7 \mathrm{~Hz}$ may likely be due to a low-level jet effect.

Overall, the application of the methodology to the full-scale data was not successful; however, the issue lies in the interpretation of the time lag versus frequency information which does exhibit the desired overall trend. It should be noted that only 2 of the 96 sensors were analyzed due to the time constraints in the program (see Section 3.8 for more discussion).

\subsubsection{Brief Summary}

An automated methodology for estimating the velocity profile from surface pressure sensors was successfully established and applied to wind tunnel boundary layer data. The limited application attempt to the field data was not successful, but this is mostly due to the effort to use the same methodology without modification. The general trends in the field 


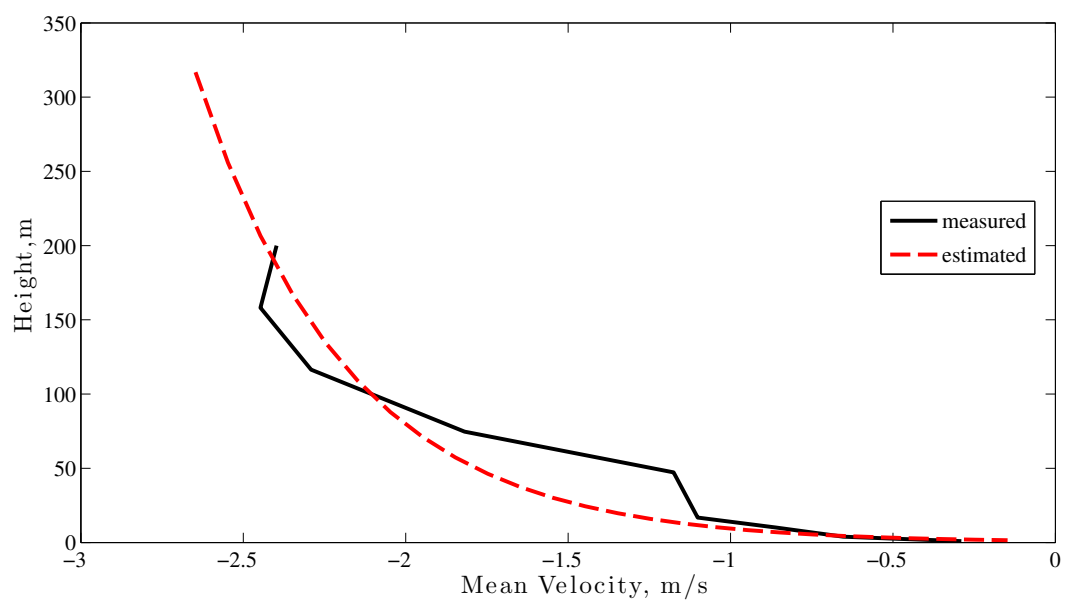

(a) $u$-Profile

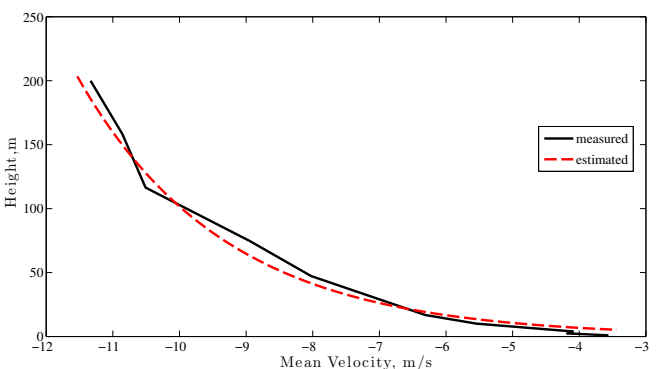

(b) $v$-Profile

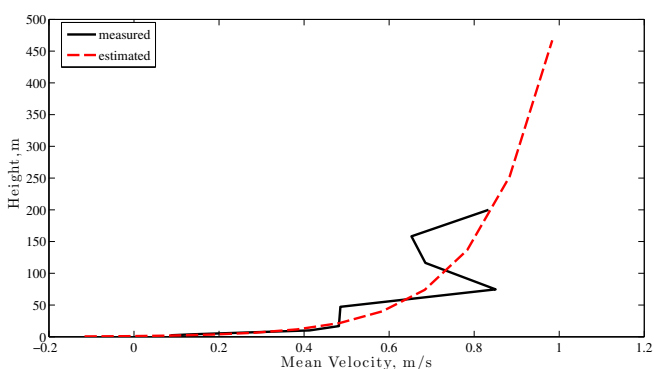

(c) $w$-Profile

Fig. 3.56. Comparison of meteorology tower measured velocity profiles in three directions and those estimated using two ground pressure sensors during the field tests. 
data suggest the information is available for a successful estimation. With the conclusion of the RTC field test, a significant data set now exists to pursue this further.

\subsection{Gust Detection and Tracking}

Wind gusts can be described as events with large spatial scales that occur over short time scales. Therefore, from a ground-based pressure perspective, these events tend to exhibit high-frequency content as well as high correlation over a large area. Based on this observation, gust detection could be performed through dynamic systems analysis. In particular, orthogonal-mode-based analysis was applied in the current work to demonstrate how these methods might provide a gust detection and tracking system using the ground based array. This approach was taken specifically based on the observations of pressure waves propagating across the infrasound arrays as shown in Fig. 3.2.

\subsubsection{Observation of Length Scales Using POD}

According to Dr. Shields' findings, a key to determining the relationship between ground based measurements and elevated wind speed estimations is understanding the spatial length scales in the surface-pressure correlations in order to relate them to spatial scales of the atmospheric boundary layer turbulence. A first look at the overall surface-pressure spatial scales was computed using proper orthogonal decomposition (POD) which is well suited to obtained the dominant empirical modes for a non-homogeneous system ${ }^{[7]}$.

In short, the POD suggests a series representation of the surface-pressure, $P(\vec{x}, t)$, such that

$$
P(\vec{x}, t)=\sum_{s} \alpha^{(s)}(t) \phi^{(s)}(\vec{x})
$$

The empirical eigen-modes, $\phi^{(s)}$, are determined from the cross-correlation matrix of the pressure sensors through solution of the integral eigenvalue problem

$$
\int_{\Omega} R_{p p}\left(\vec{x}, \vec{x}^{\prime}\right) \phi^{(s)}\left(\vec{x}^{\prime}\right) d \vec{x}^{\prime}=\lambda^{(s)} \phi^{(s)}(\vec{x}) .
$$

The temporal expansion coefficients, $\alpha^{(s)}(t)$, can be obtained through projection of the original signal onto the span of the empirical eigen-modes

$$
\alpha^{(s)}(t)=\int_{\Omega} P(\vec{x}, t) \phi^{(s)}(\vec{x}) d \vec{x} .
$$

Fig. 3.57 shows four dominant POD modes determined from 1-hour-long data sets for midnight and noon conditions on Tuesday of the Locke Station experiment. The plots show that the spatial scales represented by the midnight modes are larger in size relative to those for daytime conditions. This is a key result because of the night/day relation to stable/unstable conditions in the atmosphere. The unstable conditions during the day yield smaller scale turbulence due to the buoyant motion. At night, the more stable conditions typically give rise to complications in simulation and analysis because of the lack of "turbulence." However, gusting events in stable conditions are one of the most dangerous problems for wind turbines. Therefore, demonstrating large spatial correlations that could be used to track large scale gusting events in a stable atmosphere is a key result. 

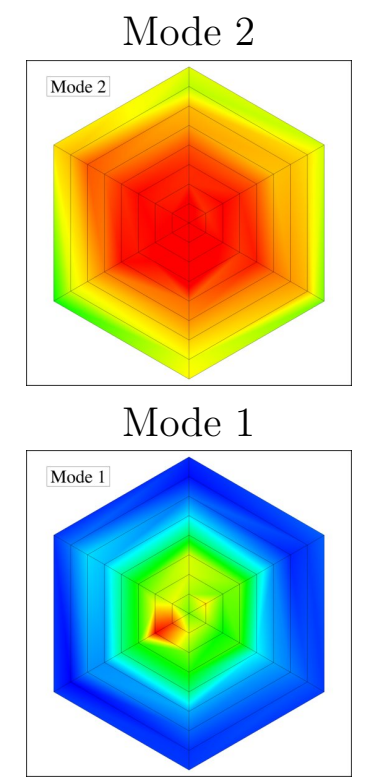
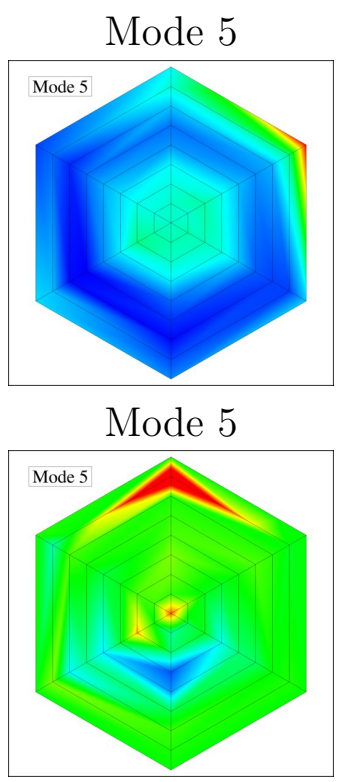
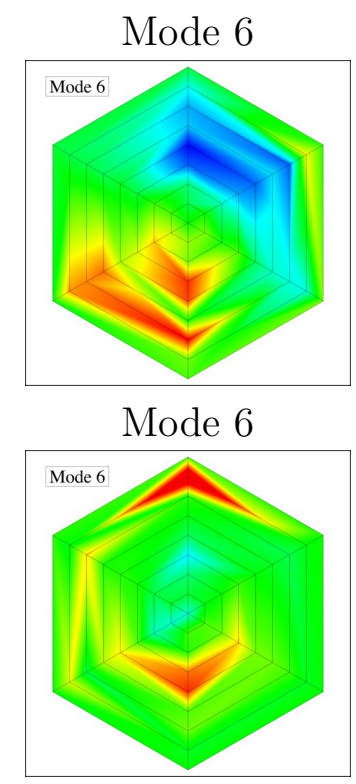
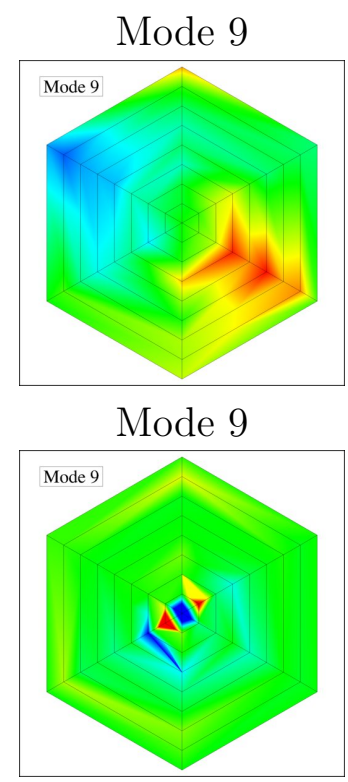

Fig. 3.57. Comparison of POD eigen-modes obtained for midnight and noon conditions: top row - midnight; bottom row - noon.

\subsubsection{DMD Tracking}

A method for both detecting and tracking large-scale coherent events was developed based on the Dynamic Mode Decomposition (DMD). The DMD is a low-dimensional method that describes the dynamic evolution of a flow system by a set of coherent modes with characteristic stability and frequency. The dynamical behavior of each mode is immediately known, and the flow field can then be modeled by these modes. As such, DMD is well-suited to applications in localized forecasting, particularly when specific coherent behavior must be extracted from the overall flow field.

The basis for the dynamic mode decomposition is the more general Koopman mode analysis. For a finite, non-linear dynamical system, the evolution of any function of the system, called an observable, can be described as a mapping by the infinite, linear Koopman operator. ${ }^{[20]}$ In this case, the system will be the near-earth boundary layer, and the observables will be measured fluid properties, such as velocity, temperature, or pressure.

The dynamic mode decomposition is the Koopman mode analysis for a discrete linear system in regular intervals. When applied to a non-linear system, such as the atmospheric boundary layer, the dynamic modes are a linear tangent approximation to the theoretical non-linear Koopman modes. ${ }^{[23]}$ The dynamic mode decomposition has been applied previously to image-based flow visualization data from controlled experiments. ${ }^{[22-25]}$

The algorithm follows the procedure as developed by Schmid, including the singular value decomposition preprocessing step for robustness. ${ }^{[23]}$ Here the algorithm is summarized to illustrate the unique problems in decomposing the near-earth atmospheric boundary layer system.

Any $M$ flow field measurements at a certain time step $i$ are first arranged into a snapshot vector, $\mathbf{v}_{i}$. A snapshot sequence, $\mathbf{V}_{1}^{N}$, then is a matrix of $N$ snapshot vectors taken at regular intervals, where the subscript denotes the first time step and the superscript denotes the last. 
It is assumed that a linear mapping $\mathbf{A}$ exists such that for any time step $i$,

$$
\mathbf{v}_{i+1}=\mathbf{A} \mathbf{v}_{i}
$$

Therefore, for two adjacent snapshot sequences $\mathbf{V}_{1}^{N-1}$ and $\mathbf{V}_{2}^{N}$ such that

$$
\mathbf{V}_{1}^{N-1}=\left[\begin{array}{lllll}
\mathbf{v}_{1} & \mathbf{v}_{2} & \cdots & \mathbf{v}_{N-2} & \mathbf{v}_{N-1}
\end{array}\right]
$$

and

$$
\begin{gathered}
\mathbf{V}_{2}^{N}=\left[\begin{array}{lllll}
\mathbf{v}_{2} & \mathbf{v}_{3} & \cdots & \mathbf{v}_{N-1} & \mathbf{v}_{N}
\end{array}\right] \\
\mathbf{V}_{2}^{N}=\mathbf{A} \mathbf{V}_{1}^{N-1}
\end{gathered}
$$

The dynamic eigenvalues and modes are equivalent in theory to the eigenvalues and eigenvectors of the matrix A. However, in the case of experimental data, the underlying linear transformation is unknown. The following matrix-free method circumvents this problem. Schmid supposes that, after some number $N-1$ of snapshot vectors, the underlying physical dynamics will be fully captured, and the next snapshot vector can be expressed approximately as the linear combination of the previous vectors.

$$
\mathbf{v}_{N}=a_{1} \mathbf{v}_{1}+a_{2} \mathbf{v}_{2}+\cdots+a_{N-1} \mathbf{v}_{N-1}+\mathbf{r}
$$

where $\mathbf{r}$ is the residual vector. As $N$ increases in a purely linear, stationary system, it is expected that the norm of $\mathbf{r}$ will approach zero. However, the atmospheric boundary layer is both non-linear and non-stationary, and the surface pressures are the only observables, so the residual vector norm will be significant for all $N \leq M$. While the method developed in Schmid (2010) ${ }^{[23]}$ will retain its mathematical form, there is a subtle difference in the underlying assumption: While the overall dynamics may not yield to linear stationary approximation, within the fluid system, there exist certain coherent, approximately-linear dynamics. Let $N>M$, so that Equation (3.23) is overdetermined. In this case, $\mathbf{r}$ will be zero, and the coefficients $\mathbf{a}=\left[\begin{array}{lll}a_{1} & \cdots & a_{N-1}\end{array}\right]^{T}$ will be found from the least-squares solution of

$$
\mathbf{v}_{N}=\mathbf{V}_{1}^{N-1} \mathbf{a} .
$$

As more snapshot vectors are added to the sequence, the linear combination of spatial and/or temporal incoherence in the measurements will decrease in magnitude, and $\mathbf{v}_{N}$ will approach the linear combination of only the coherent dynamics.

By defining the companion matrix $\mathbf{S}$ as

$$
\mathbf{S} \equiv\left[\begin{array}{ccccc}
0 & \cdots & 0 & a_{1} \\
1 & & & & a_{2} \\
0 & \ddots & & \vdots & \vdots \\
\vdots & & 1 & 0 & a_{N-2} \\
0 & \cdots & 0 & 1 & a_{N-1}
\end{array}\right]
$$

the snapshot sequence matrices $\mathbf{V}_{1}^{N-1}$ and $\mathbf{V}_{2}^{N}$ can be related by

$$
\mathbf{V}_{2}^{N}=\mathbf{V}_{1}^{N-1} \mathbf{S}
$$




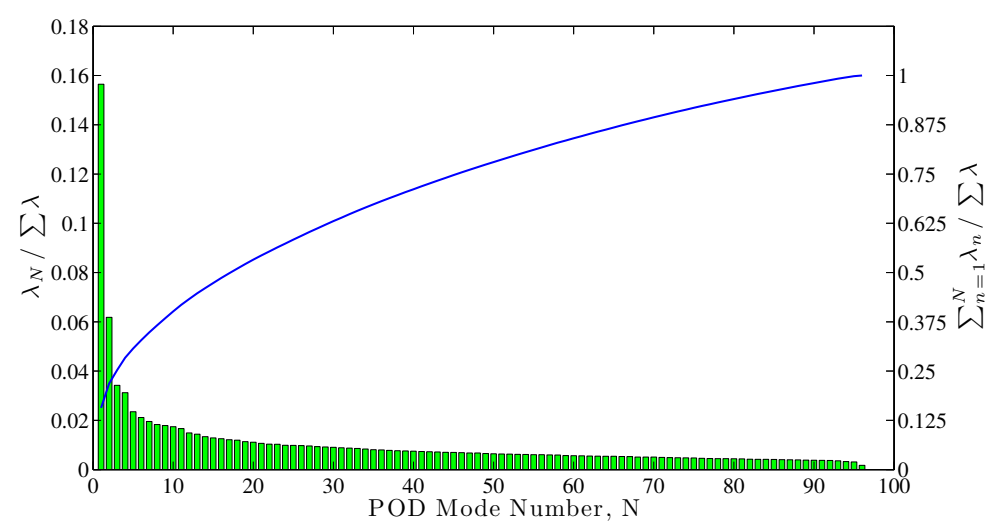

Fig. 3.58. POD basis distribution for Reese Technology Center field site data on Tuesday, July $21^{s t}, 2011$ at 2300 UTC $(6 \mathrm{pm})$.

Evidently, from Equation (3.22), the dynamic mapping A can be related to the companion matrix $\mathbf{S}$. In order to ensure a robust algorithm, the singular value decomposition of $\mathbf{V}_{1}^{N-1}$ is computed according to

$$
\mathbf{V}_{1}^{N-1}=\mathbf{U} \Sigma \mathbf{W}^{\dagger}
$$

as suggested for potentially noisy experimental data by Schmid.

Incidentally, the SVD computes the proper orthogonal decomposition of the snapshot sequence. The $M \times N$ diagonal matrix $\boldsymbol{\Sigma}$ contains the POD eigenvalues, and the $M \times M$ unitary matrix $\mathbf{U}$ contains the proper orthogonal modes. As such, this preprocessing step imparts additional information about the coherence of the system and its suitability to lowdimensional analysis. The first $R$ modes of the POD are selected by some method, and an $R \times R$ square diagonal matrix $\mathbf{D}$ is extracted from $\boldsymbol{\Sigma}$ to give the approximation

$$
\mathbf{V}_{1}^{N-1} \approx \mathbf{U}_{R} \mathbf{D} \mathbf{W}_{R}^{\dagger}
$$

Combining Equations (3.22) and (3.26) and substituting this reduced SVD, A can be related to $\mathbf{S}$ by

$$
\mathbf{U}_{R}^{\dagger} \mathbf{A U}_{R}=\mathbf{U}_{R}^{\dagger} \mathbf{V}_{2}^{N} \mathbf{W}_{R} \mathbf{D}^{-1}=\tilde{\mathbf{S}}
$$

where $\tilde{\mathbf{S}} \equiv\left(\mathbf{W}_{R} \mathbf{D}^{-1}\right)^{-1} \mathbf{S}\left(\mathbf{W}_{R} \mathbf{D}^{-1}\right)$. Since they are related by a similarity transformation, the eigenvalues of $\tilde{\mathbf{S}}$ are some of the eigenvalues of $\mathbf{A}$. For every $\mathbf{y}_{i}$, an eigenvector of $\tilde{\mathbf{S}}$, $\mathbf{U}_{R} \mathbf{y}_{i}$ is an eigenvector of $\mathbf{A}$. As a consequence, using this method, the dynamic modes are automatically expressed in the POD basis.

The dynamic mode decomposition was applied to the MRT-TT data from the Reese Technology Center deployment. The original $200 \mathrm{~Hz}$ time series was filtered and subsampled at $20 \mathrm{~Hz}$, and different snapshot sequence lengths were investigated. The POD basis was used as a rough criterion for the time interval over which the DMD was applied. In most cases, an interval of 2-5 minutes yielded a POD basis with over $25 \%$ of the relative eigenvalue magnitude contained in the first ten modes. A typical POD eigenvalue distribution for data taken at 2300 UTC on July 21st is shown in Fig. 3.58. The decomposition was over an interval of 250 seconds. 


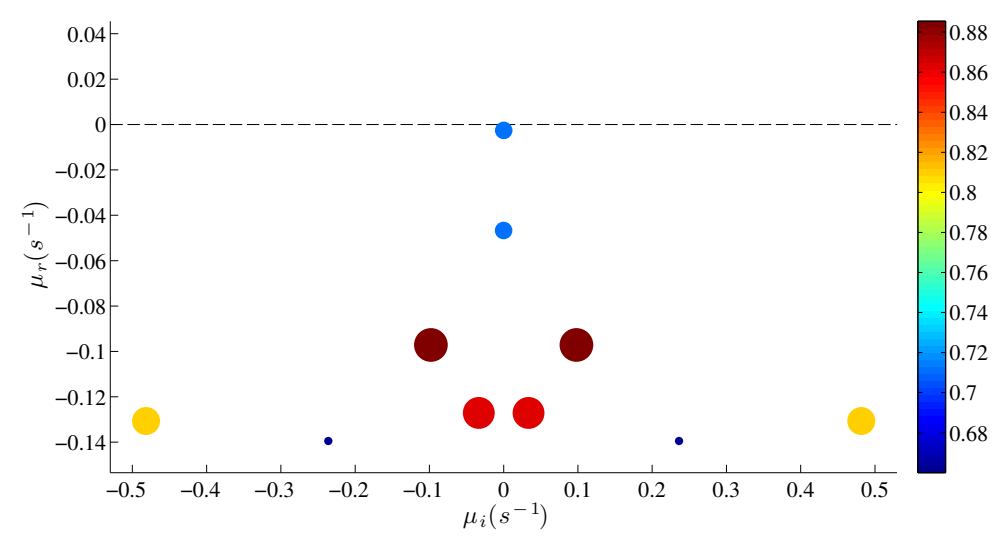

Fig. 3.59. DMD spectrum for Reese Technology Center field site data on Tuesday, July $21^{s t}$, 2011 at 2300 UTC (6 pm). The magnitude is the norm of each mode.

The dynamic mode spectrum for the same data, with POD basis restricted to the first 10 modes, is shown in Fig. 3.59. The eigenvalues $\lambda$ have been mapped onto the stability plane by $\mu=\log (\lambda) / \Delta t$, where $\mu_{r}$ and $\mu_{i}$ are the real and imaginary components, respectively. The real component is related to the stability of the mode as a characteristic time of growth or decay, while the imaginary component is the frequency of the mode. The color and size of each point in the spectrum is associated with the coherence, given by the vector norm of each DMD mode. Since the modes are expressed in the orthonormal POD basis, the norm has a maximum of 1 .

Each mode can be considered a complex-valued distribution of pressures on the original transducer array that evolves in time as the product with its associated eigenvalue. The spectrum in Fig. 3.59 exhibits several coherent, low-frequency, stable modes. Taking the first mode as an example, the vector is stepped through time by a product with its eigenvalue, and the real component at each time step is plotted on a representation of the original pressure transducer array. The result is plotted at four illustrative times in Fig. 3.60. Evidently, this mode is associated with a localized pressure disturbance moving across the array from South to North. In the figure, the grid crossings are the locations of the pressure sensors which are physically 4 meters apart. This suggests that the disturbance represented by Mode 1 moves at approximately $2.5 \mathrm{~m} / \mathrm{s}$ from South to North. This agrees with the wind speed and direction from Figures 3.37 and 3.39. Modes with similar behavior can be extracted from other decompositions at different times.

To validate this method, a synthetic data series was constructed by creating a disturbance with a Gaussian spatial shape and allowing this disturbance to move across the array at a given speed.

\subsubsection{DMD of a Synthetic, Transient Pressure Distribution}

Dynamic modes have been shown to exist associated with propagating localized pressure disturbances. However, the modes that exhibit this qualitative behavior are not spectrally consistent, and must be identified by inspection for each decomposition of experimental data. 


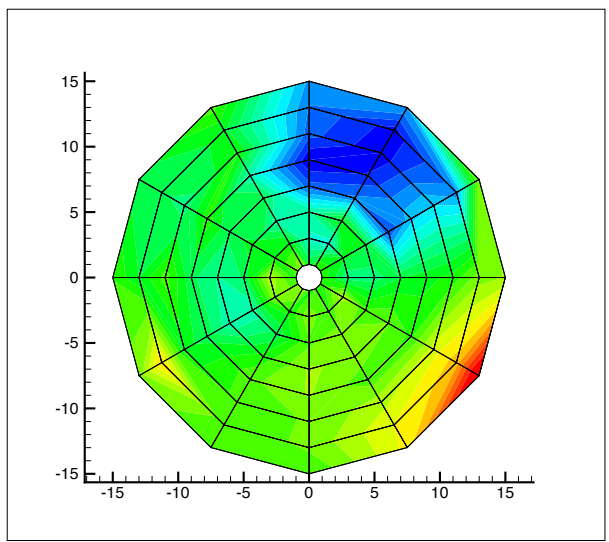

(a) $t=0 \mathrm{~s}$

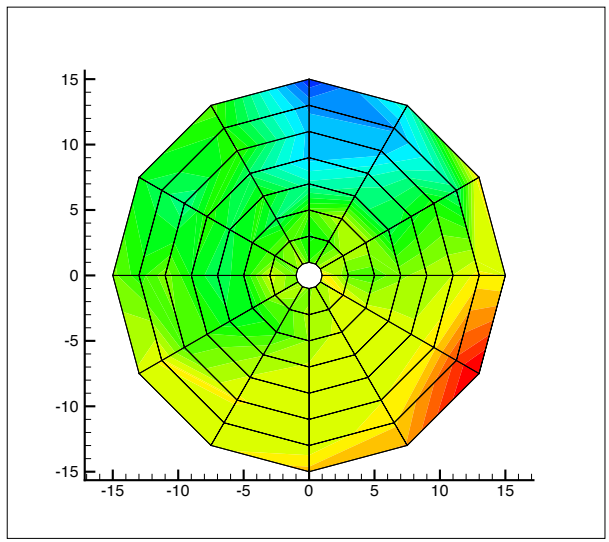

(c) $t=4 \mathrm{~s}$

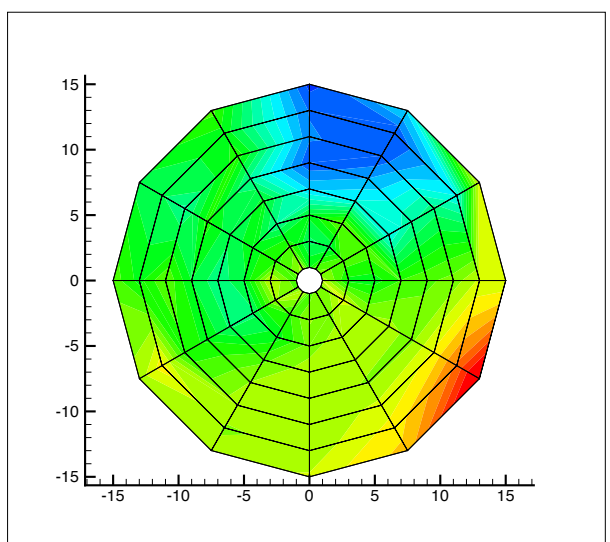

(b) $t=2 \mathrm{~s}$

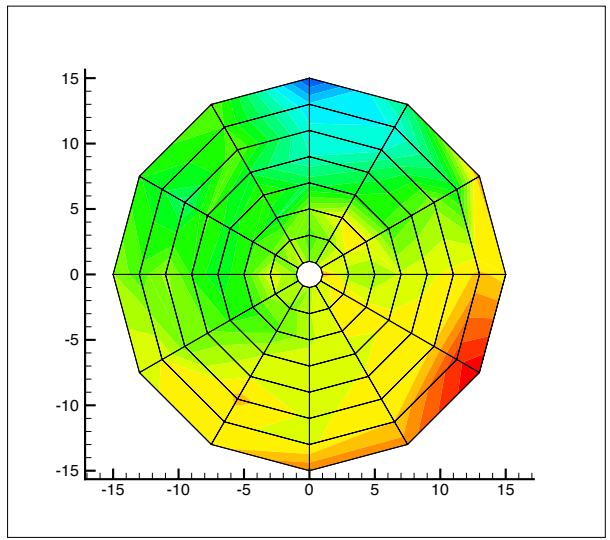

(d) $t=6 \mathrm{~s}$

Fig. 3.60. Evolution of dynamic mode 1 for the decomposition of Reese Technology Center field site data on Tuesday, July $21^{s t}, 2011$ at 2300 UTC (6 pm). North is the $+y$-direction. 
In order to characterize the spectra of such localized pressure distributions, a synthetic data set was generated and decomposed using the same DMD algorithm.

The pressure disturbance due to a billow or gust can be idealized as a propagating twodimensional circular Gaussian distribution,

$$
p(x, y, t)=\exp \left\{-\frac{\left(x-x_{0}-u_{x} t\right)^{2}+\left(y-y_{0}-u_{y} t\right)^{2}}{2 \sigma^{2}}\right\},
$$

where $\left(x_{0}, y_{0}\right)$ is the initial position of the center of the disturbance, and $\left(u_{x}, u_{y}\right)$ is the disturbance velocity. The effective spatial diameter of the disturbance is specified by the $2-\sigma$ width, so that $D \equiv 4 \sigma$. Anticipating comparison with the modal spectrum, the velocity is not specified directly. Instead, an effective period is specified, the time for the center of the distribution to cross the simulated sensor array.

Several synthetic time series were generated on a simulation of the small aperture array from the Reese Technology Center experimental campaign, using the same acquisition rate of $20 \mathrm{~Hz}$. For comparison, a disturbance was chosen with approximately the same diameter as the mode resolved in Figure 3.60. Three snapshots of the time history of this synthetic disturbance are plotted in Figure 3.63. The disturbance was propagated off-center to avoid introducing any symmetries that might affect the generality of the decomposition.

The DMD was applied over a 50-second window centered about the point of nearest approach for the disturbance. A POD basis of 11 non-negligible modes was extracted, and subsequently, 11 dynamic modes were obtained. The eigenvalue spectrum is shown in Figure 3.61. The frequencies of the non-stationary modes, which appear in conjugate pairs, are related by integer multiples. These modes are relatively stable, with characteristic decay times on the order of 20 times the window extent. A combination of continuous, constantfrequency modes at harmonic frequencies is reminiscent of a Fourier transform of a transient function, and in fact, for dynamics evolving on a attractor, the DMD can be shown to be equivalent to a discrete Fourier transform. ${ }^{[20]}$

The real component of each mode conjugate pair is shown in Figure 3.62, along with the associated angular frequency. For each successive harmonic frequency, the spatial wavelength of the modal distribution also increases, following a phase velocity relationship, $v=\omega / k$. These modes propagate in the same direction as the Gaussian disturbance itself.

Finally, to verify the decomposition, the dynamic modes were used to reconstruct the time history. First, the modes are weighted by their projection onto the first time step in the synthetic data. The time evolution of each complex mode is given by the product with the respective complex eigenvalue. By superimposing the modes at each time step, the reconstruction shown in Figure 3.64 was obtained. The reconstructed disturbance leads the original slightly in time, but is otherwise in good agreement with the original data. If the modes are weighted by a projection onto a later time step, when the disturbance has fully entered the array, the observed discrepancy disappears.

The synthetic decomposition demonstrates that transient disturbances will not decompose into a single mode, but rather, a series of harmonic modes. In future work, spectra of experimental data will be examined for families of modes that might be superimposed to obtain transient signals, instead of individual, periodic modes. 


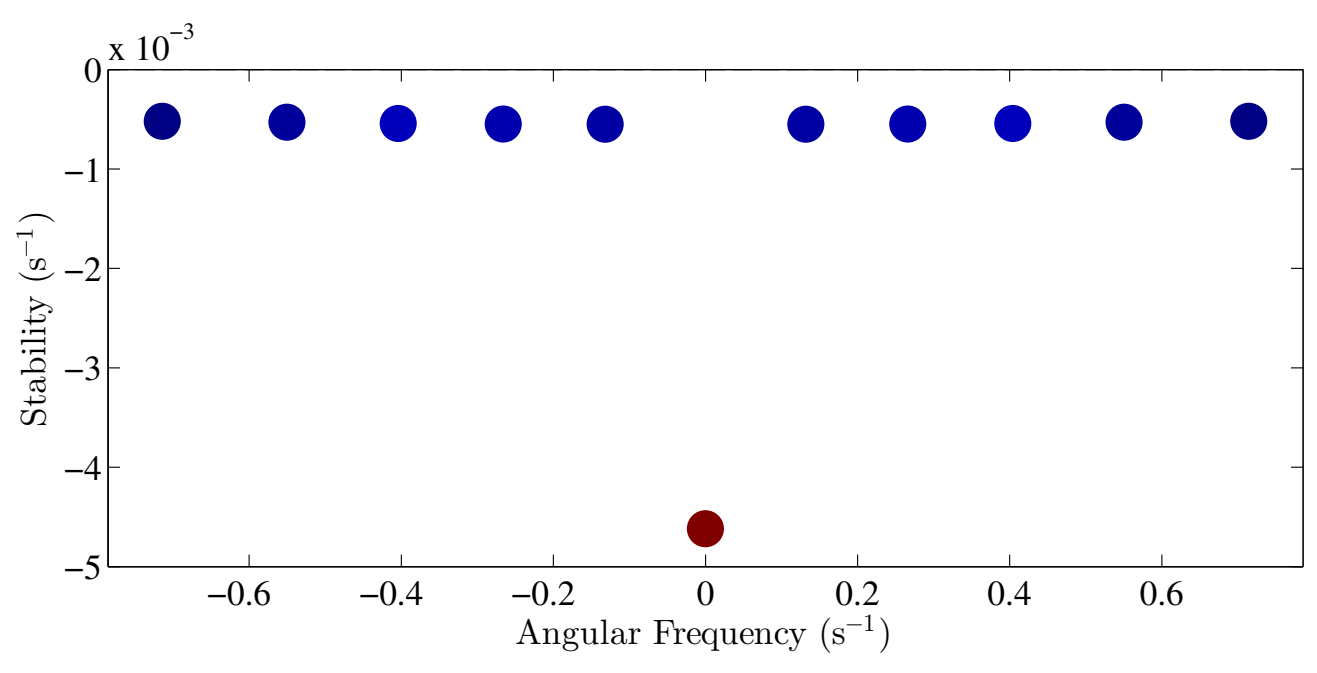

Fig. 3.61. The dynamic mode spectrum for the synthetic Gaussian distribution.

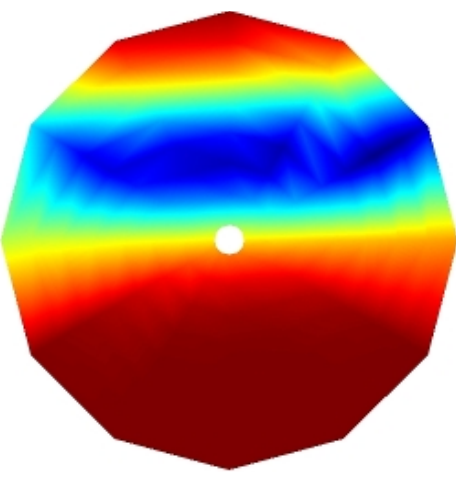

(a) $\mu_{1, i}=0.0 \mathrm{~s}^{-1}$

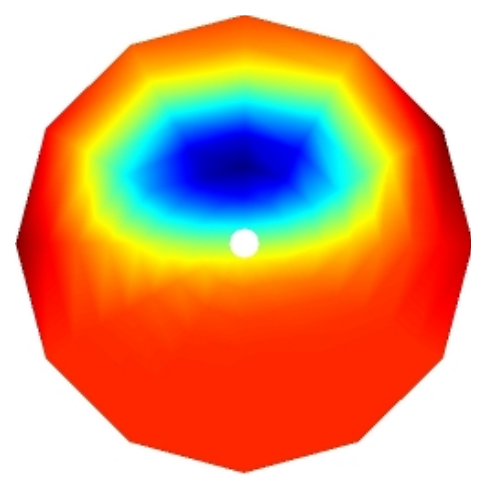

(d) $\mu_{6 / 7, i}= \pm 0.132 \mathrm{~s}^{-1}$

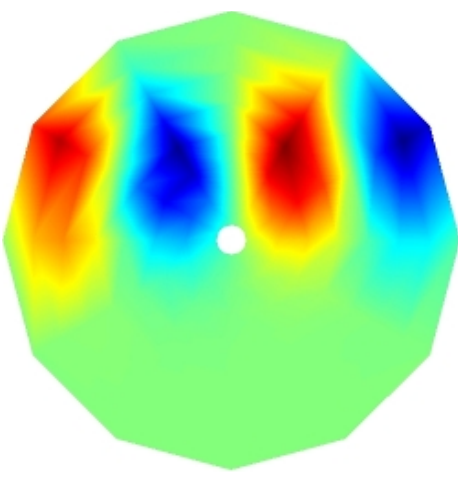

(b) $\mu_{2 / 3, i}= \pm 0.404 \mathrm{~s}^{-1}$

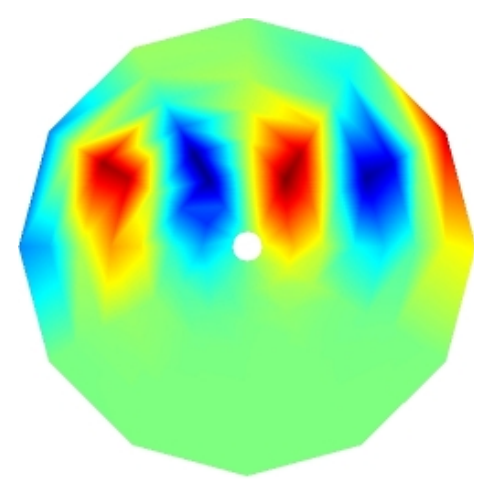

(e) $\mu_{8 / 9, i}= \pm 0.550 \mathrm{~s}^{-1}$

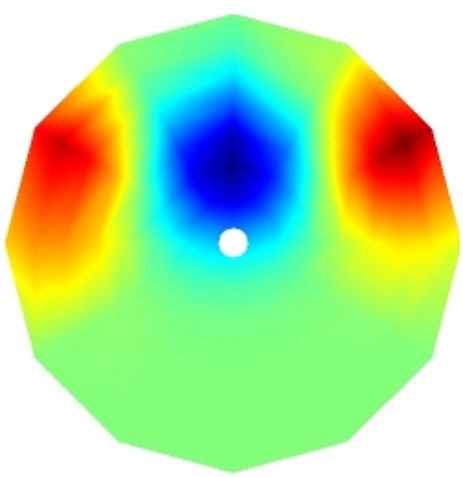

(c) $\mu_{4 / 5, i}= \pm 0.266 \mathrm{~s}^{-1}$

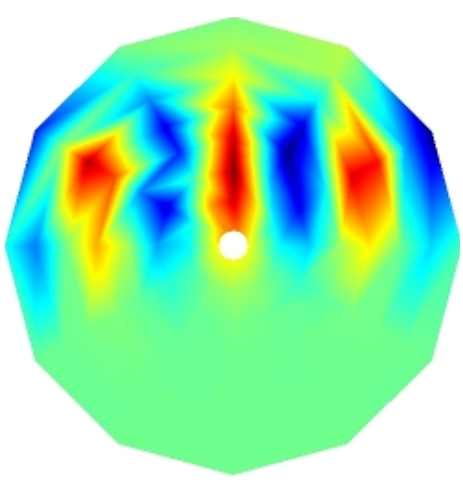

(f) $\mu_{10 / 11, i}= \pm 0.714 \mathrm{~s}^{-1}$

Fig. 3.62. The dynamic modes obtained from a decomposition of the synthetic Gaussian distribution. 


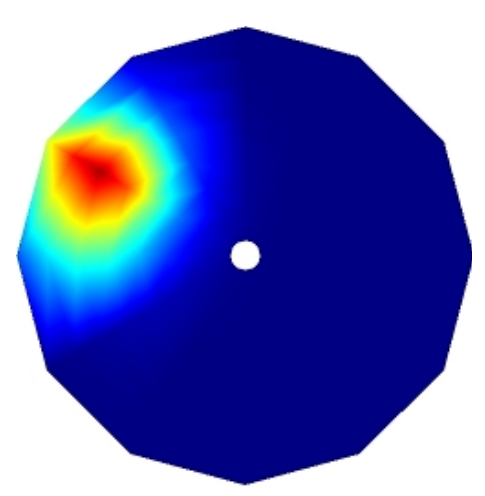

(a) $t=15 \mathrm{~s}$

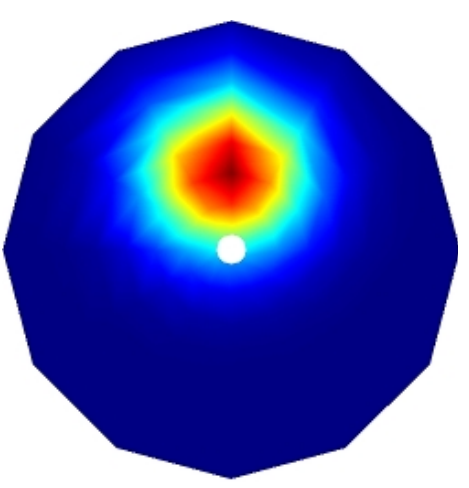

(b) $t=25 \mathrm{~s}$

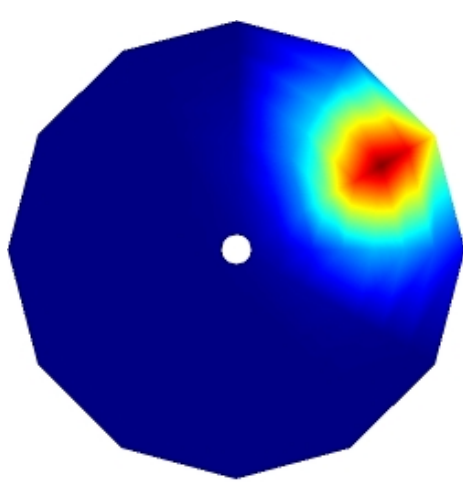

(c) $t=35 \mathrm{~s}$

Fig. 3.63. Three snapshots of the synthetic time series from the propagating Gaussian disturbance.

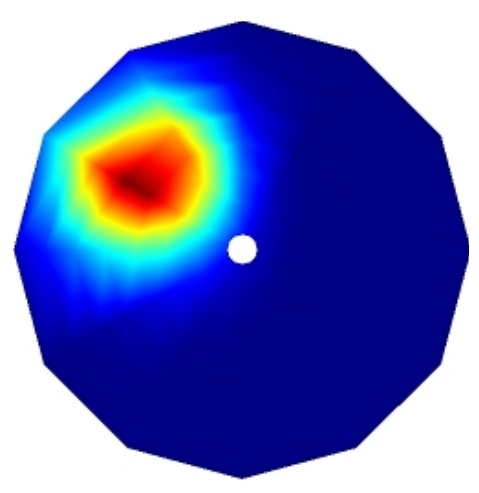

(a) $t=15 \mathrm{~s}$

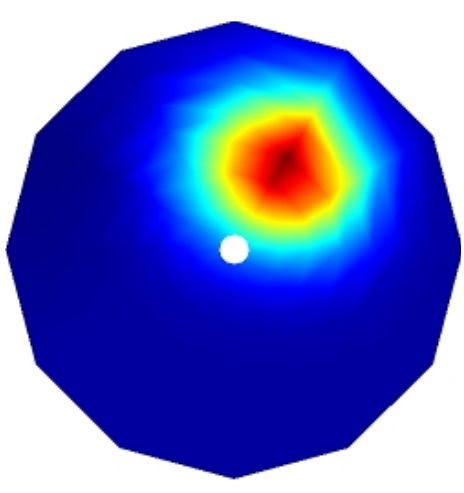

(b) $t=25 \mathrm{~s}$

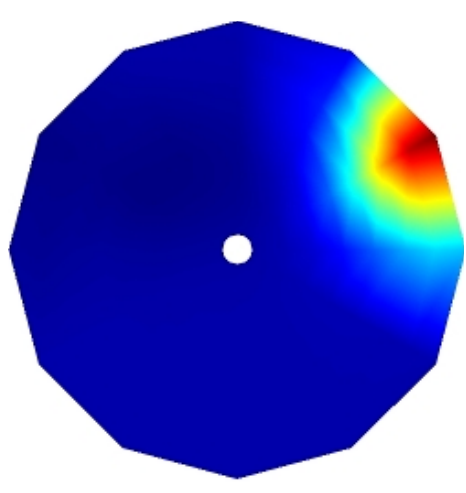

(c) $t=35 \mathrm{~s}$

Fig. 3.64. Three snapshots of the time series reconstructed from superposition of the dynamic modes. 


\subsection{Conclusions and Future Work}

The concept of utilizing infrasound pressure sensors in a wireless array to sense the local wind conditions around a wind turbine was explored. Prior experience and theoretical analyses led to the main hypothesis that an array of suitable pressure transducers distributed on the ground could be used to predict the time-dependent velocity profile in the nearEarth boundary layer. To prove the hypothesis in general, it was necessary to evaluate two questions: (a) Can a ground-based pressure measurement system yield an estimation of the vertical velocity profile, and (b) is an infrasonic pressure array capable of detecting and tracking gust-type events?

To explore the main hypothesis, both wind tunnel experiments and large-scale field deployments were carried out to collect data with simultaneous measurements of the surface pressure fluctuations and the velocities in the boundary layer. Very few atmospheric measurement campaigns have measured the ABL (velocity, temperature, pressure) simultaneously with a large infrasonically sensitive surface pressure array. Therefore, a bulk of the effort was focused on acquiring this data for archival purposes. The field deployment at the Reese Technology Center successfully provided the synchronous data set necessary for evaluating low-frequency pressure/velocity coupling in the ABL.

Analysis of the wind tunnel data successfully yielded a methodology whereby the mean vertical velocity profile could be estimated from the surface pressure coherence. Application of the dynamic mode decomposition (DMD) to the RTC field data successfully isolated and showed the propagation of a large disturbance moving across the array at a speed and direction consistent with the wind measured at the site. These two accomplishments serve to prove in a general sense that the main hypothesis is true. Still, the development of these two tools into a complete hands-off system will required additional work.

One of the drawbacks of the current work is that the velocity profile estimation, while effective, still depended on the knowledge of the free-stream velocity present outside the boundary layer. It is likely that this dependence could be removed by utilizing past atmospheric research results. In particular, Klewicki et al. ${ }^{[9]}$ suggest that the RMS amplitude of the pressure fluctuations could be used to estimate the boundary layer thickness using Equation (3.3). Additional research is needed to determine a suitable method for using $p_{r m s}$ in this manner to yield the velocity at the edge of the boundary layer. Future work would utilize the data in the RTC field deployment to explore these relationships in greater depth.

\section{Bibliography}

[1] Bass, H.; Raspet, R.; \& Messer, J. (1995) "Experimental Determination of Wind Speed and Direction Using a Three-Microphone Array." J. of the Acoustical Society of America, Vol. 97:695-696.

${ }^{[2]}$ Bull, M. K. (1967) "Wall Pressure Fluctuations Associated with a Subsonic Turbulent Boundary Layer." Journal of Fluid Mechanics, Vol. 28:719-754.

[3] Clifton, A. (2012) personal communication.

[4] Dillion, K.; Howard, W.; \& Shields, F. D. (2007) "Advances in Distributed Arrays for Detection of Infrasonic Events." J. of the Acoustical Society of America, Vol. 122:2960. 
[5] George, W.; Beuther, P.; \& Arndt, R. (1984) "Pressure Spectra in Turbulent Free Shear Flows." Journal of Fluid Mechanics, Vol. 148:155-191.

[6] Gravante, S.; Naguib, A.; Wark, C.; \& Nagib, H. (1998) "Characterization of the Pressure Fluctuations Under a Fully Developed Turbulent Boundary Layer." AIAA Journal, Vol. 36(10):1808-1816.

${ }^{[7]}$ Holmes, P.; Lumley, J.; \& Berkooz, G. (1998) Turbulence, Coherent Structures, Dynamical Systems and Symmetry. Cambridge Monographs on Mechanics. Cambridge University Press.

${ }^{[8]}$ Kelley, N. D.; Jonkman, B. J.; Scott, G. N.; Bialasiewicz, J. T.; \& Redmond, L. S. (2005) "The Impact of Coherent Turbulence on Wind Turbine Aeroelastic Response and Its Simulation." National Renewable Energy Laboratory, Conference Paper NREL/CP500-38074.

${ }^{[9]}$ Klewicki, J. C.; Priyadarshana, P. J. A.; \& Metzger, M. M. (2008) "Statistical Structure of the Fluctuating Wall Pressure and its In-Plane Gradients at High Reynolds Number." Journal of Fluid Mechanics, Vol. 609:195-220.

[10] Kraichnan, R. (1956) "Pressure Fluctuations in Turbulent Flow over a Flat Plate." J. of the Acoustical Society of America, Vol. 28(3):378-390.

${ }^{[11]}$ Le, H.; MoIn, P.; \& KIm, J. (1997) "Direct Numerical Simulation of Turbulent Flow Over a Backward-Facing Step." Journal of Fluid Mechanics, Vol. 330:349-374.

${ }^{[12]}$ Marusic, I.; Mathis, R.; \& Hutchins, N. (2010a) "Predictive Model for WallBounded Turbulent Flow." Science, Vol. 329:193-196.

${ }^{[13]}$ Marusic, I.; McKeon, B. J.; Monkewitz, P. A.; Nagib, H. M.; Smits, A. J.; \& Sreenivasan, K. R. (2010b) "Wall-Bounded Turbulent Flows at High Reynolds Numbers: Recent Advances and Key Issues." Physics of Fluids, Vol. 22(6):065103.

${ }^{[14]}$ Matsubara, M. \& Alfredsson, P. (2001) "Disturbance Growth in Boundary Layers Subjected to Free-Stream Turbulence." Journal of Fluid Mechanics, Vol. 430(1):149-168.

${ }^{[15]}$ Murray, N.; Raspet, R.; \& Ukeiley, L. (2011) "Contributions of Turbulence to Subsonic Cavity Flow Wall Pressures." Physics of Fluids, Vol. 23(1):015104:1-13.

${ }^{[16]}$ Naguib, A.; Wark, C.; \& Juckenhöfel, O. (2001) "Stochastic Estimation and Flow Sources Associated with Surface Pressure Events in a Turbulent Boundary Layer." Physics of Fluids, Vol. 13(9):2611-2626.

${ }^{[17]}$ Panton, R. L. \& Linebarger, J. H. (1974) "Wall Pressure Spectra Calculations for Equilibrium Boundary Layers." Journal of Fluid Mechanics, Vol. 65:261-287.

[18] Priestly, J. (1965) "Correlation Studies of Pressure Fluctuations on the Ground Beneath a Turbulent Boundary Layer." National Bureau of Standards, Report 8942 also M.S. Thesis, U. of Maryland, 1965. 
[19] Priestly, J. (1966) "Calculation of the Effectiveness of Infrasonic Line Microphones for Reducing Wind Noise." National Bureau of Standards, Report 9380 This report corrects a mistake in the value of alpha given in the 1965 report.

${ }^{[20]}$ Rowley, C. W.; Mezić, I.; Bagheri, S.; Schlatter, P.; \& Henningson, D. S. (2009) "Spectral analysis of nonlinear flows." Journal of Fluid Mechanics, Vol. 641:115-127.

[21] Schewe, G. (1983) "On the Structure and Resolution of Wall-Pressure Fluctuations Associated with Turbulent Boundary-Layer Flow." Journal of Fluid Mechanics, Vol. 134:311328.

[22] Schmid, P. J. (2009) "The description of fluid behavior by coherent structures." In Seventh IUTAM Symposium on Laminar-Turbulent Transition.

[23] Schmid, P. J. (2010) "Dynamic mode decomposition of numerical and experimental data." Journal of Fluid Mechanics, Vol. 656:5-28.

[24] Schmid, P. J. (2011) "Application of the dynamic mode decomposition to experimental data." Experiments in Fluids, Vol. 50:1123-1130.

[25] Schmid, P. J.; Li, L.; Juniper, M. P.; \& Pust, O. (2011) "Applications of the dynamic mode decomposition." Theoretical Computational Fluid Dynamics, Vol. 25:249259.

[26] ShiElds, F. (2005) "Low-Frequency Wind Noise Correlation in Microphone Arrays." J. of the Acoustical Society of America, Vol. 117(6):3489-96.

${ }^{[27]}$ ShiELDS, F. D. (2010) personal communication.

[28] Talmadge, C. (2009) "Piezoceramic Sensors and Infrasound." In 2009 Seismic Instrumentation Technology Symposium, Palm Springs, CA. Incorporated Resarch Institutions for Seismology (IRIS).

[29] Waxler, R. \& Gilbert, K. E. (2006) "The Radiation of Atmospheric Microbaroms by Ocean Waves." The Journal of the Acoustical Society of America, Vol. 119(5):2651-2664. 


\section{4 | LES of Gust Generated Surface Pressure}

As part of the Task 2 effort, a computation fluid dynamics (CFD) framework was developed for evaluating ground based sensor array determination of the wind velocity aloft. Two CFD efforts were pursued to this end. First, Combustion Research and Flow Technology, Inc. (CRAFT-Tech) attempted a large-eddy simulation (LES) of the 2-foot tunnel experiments conducted at the NCPA as described in 3.5.1. Second, an LES of the Atmospheric Boundary Layer (ABL) was set up and run by Jericho Cain, a graduate student at the NCPA supported by this program.

\subsection{CFD Efforts of CRAFT Tech}

The results of the CRAFT Tech effort are described in Chapter 2 of the CRAFT Tech final report which is provided in Appendix C. While some interesting results were generated, the high computation cost of resolving the small spatial scales while also generating long physical time runs was prohibitive and limited the results to qualitative observations.

\subsection{LES Simulation of the ABL}

Cain's efforts resulted in a successful implementation of an ABL simulation. The effort and current results have been condensed into Cain's Ph.D. prospectus which is currently under consideration by the graduate committee which includes Drs. Nathan Murray (PI) and Richard Raspet (Co-PI) who have been directing Cain's efforts. The main results of significance to the present work is that the wind speed suggested by surface pressure correlations exceeds the flow velocity near the ground by a factor of at least 2 . This suggests that even in the LES simulation, the convection velocity implied by the ground based correlations must be related to turbulent events aloft rather than near the ground. This result demonstrates that the LES simulation should provide a tool for further exploration of ground-based in-flow profiling methodology. The prospectus is incorporated here in its entirety on the following pages. 
Large-eddy Simulation of Surface Pressure Fluctuations Generated by Elevated Gusts.

A Prospectus for A Doctoral Dissertation

\author{
Jericho E. Cain \\ National Center for Physical Acoustics, \\ University of Mississippi.
}

(Dated: January 9, 2012) 


\section{5 | CFD Optimization of Rotor Blade Geometry}

The efforts of Task 3 were subcontracted to Combustion Research and Flow Technology, Inc., (CRAFT Tech). The goal of this effort was to develop a framework for design optimization based on multiple design objectives including noise generation. CRAFT Tech was successful in developing a methodology using a code called SCULPTOR in concert with their CRUNCH CFD flow solver and a Ffowcs Williams-Hawkings (FW-H) acoustic solver to automatically generate an optimized blade design. The design goals included maximizing the output power and minimizing the observed sound pressure level (SPL). As shown in Figure 38 of their final report, the optimizer was able to improve on the power output of the NREL BSDS blade design while also reducing the observed SPL. Complete details are provided in CRAFT Tech's final report attached as Appendix C.

The design optimization framework resulting from this effort is a significant outcome of the current program. The current industry requirements to build optimization into the design phase require that multiple design objectives can be met by the optimization method. This work demonstrated successfully the implementation of two design goals that are typically thought to be in competition with one another. In future applications, the need for low sound production could be incorporated into the airfoil design phase using this tool. 


\section{6 | Health Monitoring of Wind Turbine Systems}

The task 4 objective to develop a "health monitoring system for rotor blades with wireless flush mounted sensors" was the brainchild of Dr. Seiner. Prior to his passing, Dr. Seiner subcontracted Miltec Research \& Technology, Inc. (MRT) to perform the design and implementation of the wireless sensors and health monitoring system. MRT's work was in response to the following request for proposals published by Dr. Seiner:

Option 1: Proposers must have the capability to design an onboard, real time health monitoring system. The capability should be successfully used to design systems monitoring for damage (cracking, corrosion, bolt loosening) in other aircraft structures such as wind spars, landing gear, and various fittings.

The Option 1 proposed task is to take the proposers proven optimal design techniques and design an onboard health monitoring system. In this task the optimal locations to place sensors and actuators will be calculated to detect delaminations in a composite rotor blade. The objective of this task would be to create a system that is

1. maximally sensitive to debonding and buckling along the spar structure,

2. still able to detect damage in other areas,

3. realized with the minimal number of sensors as to minimally affect weight,

4. maximally robust to manufacturing variations, and

5. able to operate given the loss of a limited number of sensors.

The system will be designed such that it can be connected to a generic interrogation unit that will supply power and record the measurements. These measurements will then be downloaded onto a computer and processed to assess the damage condition.

The proposer's design approach shall create optimization techniques that identify sensor type and location for damage detection. Objectives in generating these designs shall include minimizing the number of sensors while ensuring a robust design in the face of error in the models. In the optimization, the allowable sensoractuator locations shall be defined to insure the resulting designs are easier to manufacture and install. The model required for the design of the system will be provided by the NCPA. The result of the proposers design shall be a set of sensor-actuator locations that will allow efficient monitoring of a wind turbine blade.

Option 2: The proposer shall use its hardware design experience to create a data collection system to support Option 1 . The system would supply power to the sensors and actuators and collect the data for processing. Different options for supplying power will be explored including solar, ambient vibrations, and electromagnetic energy collection. The data collected will most likely be transmitted wirelessly due to the inaccessibility of the location of the sensors. Due 
to the constraints of providing power for excitation, data collection, and wireless transmission, the proposer will develop a thorough power budget.

The data collection system will also be designed to support the NCPAs efforts to independently verify their wind speed measurements. To accomplish this, extra data acquisition channels will be included that can support a to-be-determined number of NCPA sensors. These channels will be sampled 5-10 times faster than the highest frequency desired to be processed. The data will also be time stamped to allow comparison to other time stamped signals. The deliverable of this option is a paper design of a system along with limited component testing depending on the needs of the NCPA.

Upon Dr. Seiner's passing, Dr. Murray found that MRT had never been provided with the blade model required for design. This put MRT's efforts significantly behind. Through communication with Joshua Paquette at Sandia National Labs, NCPA acquired the CX-100 and WindPACT blade models and passed them on to MRT. MRT was subsequently able to complete the work for Option 1 of the subcontracted effort. However, no attempt was made by MRT to conduct hardware development associated with Option 2.

At the conclusion of their work, MRT successfully demonstrated the computational design of a health monitoring system for composite blades. Their approach is unique in that they focus on monitoring the overall health of the blade by measuring changes in the dominant structural modes. While this doesn't allow for pinpointing the location of some damage it does allow for overall system monitoring with few sensors and relatively high reliability.

The complete details of MRT's efforts and findings along with their work to support Task 2 efforts are included in their final report which is attached as Appendix D. 


\section{Appendix A}

Hyperion Technology Group, Inc., IFS-3000 Infra-Sound Sensor

PAGE A-1

Final Report | DOE Award No. DE-EE0003269 


\section{IFS-3000 Series Analog Infrasound Sensor}

\section{Features:}

Modular Design

Ruggedized Construction

Differential Output

Low Power Consumption

Stable Operation

Chemical, Water, Dirt and Weather Resistant

Multiple Versions for Various Applications

Sensitivity Selectable at Time of Order

Excellent Sensor-To-Sensor Matching

Custom Configurations Available

Blast Wave Version Available

Seismically Decoupled Version Available

Applications:

Violent Convective Storm Data Collection

Anthropogenic Signal Collection

Natural Infrasound Source Data Collection

Atmospheric Structure / Propagation Study
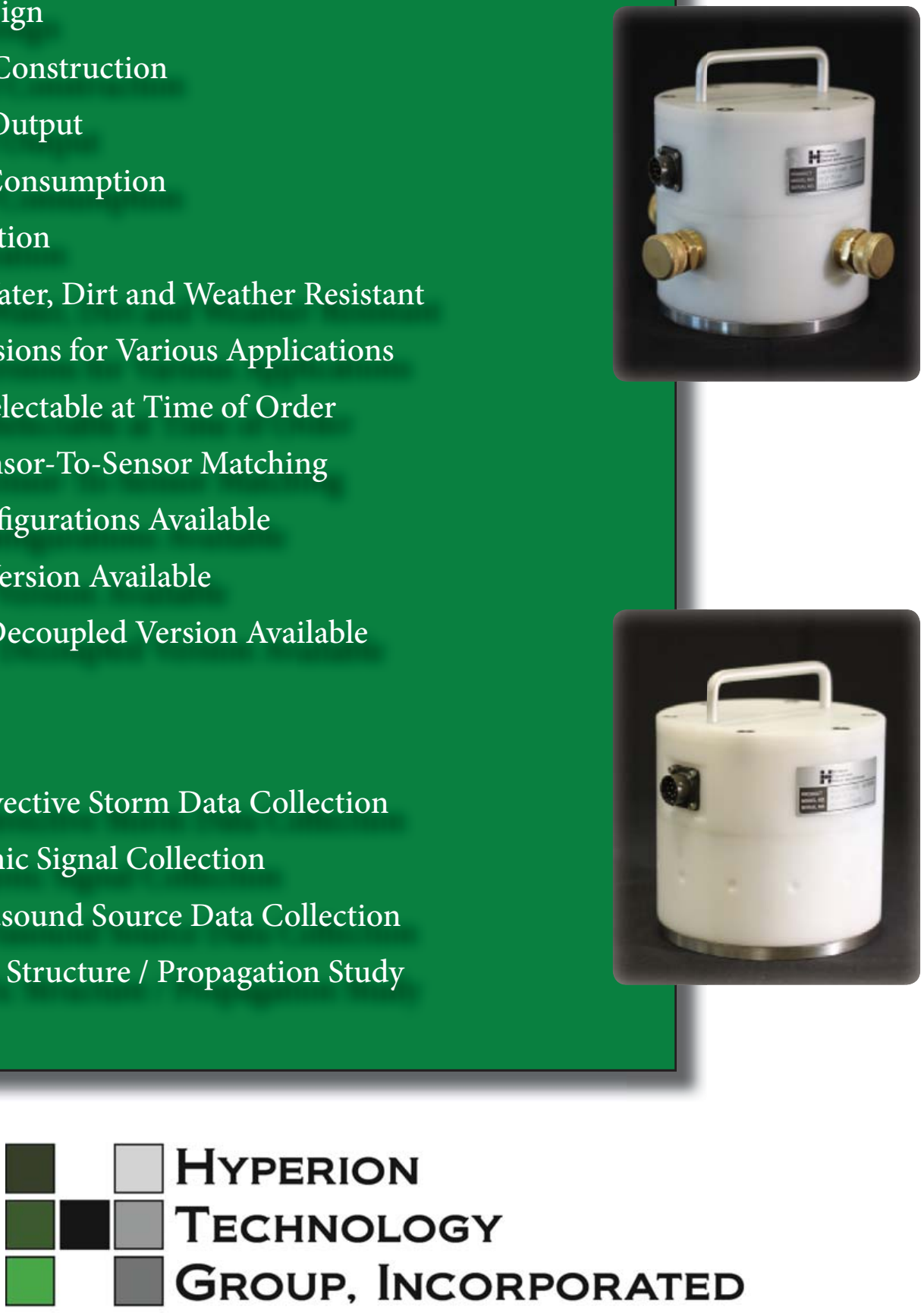

3248 West Jackson, Tupelo, Mississippi 38801-6735 662.823.0600 http://www.hyperiontechgroup.com 
Appendix A | Hyperion Technology Group, Inc., IFS-3000 Infra-Sound Sensor

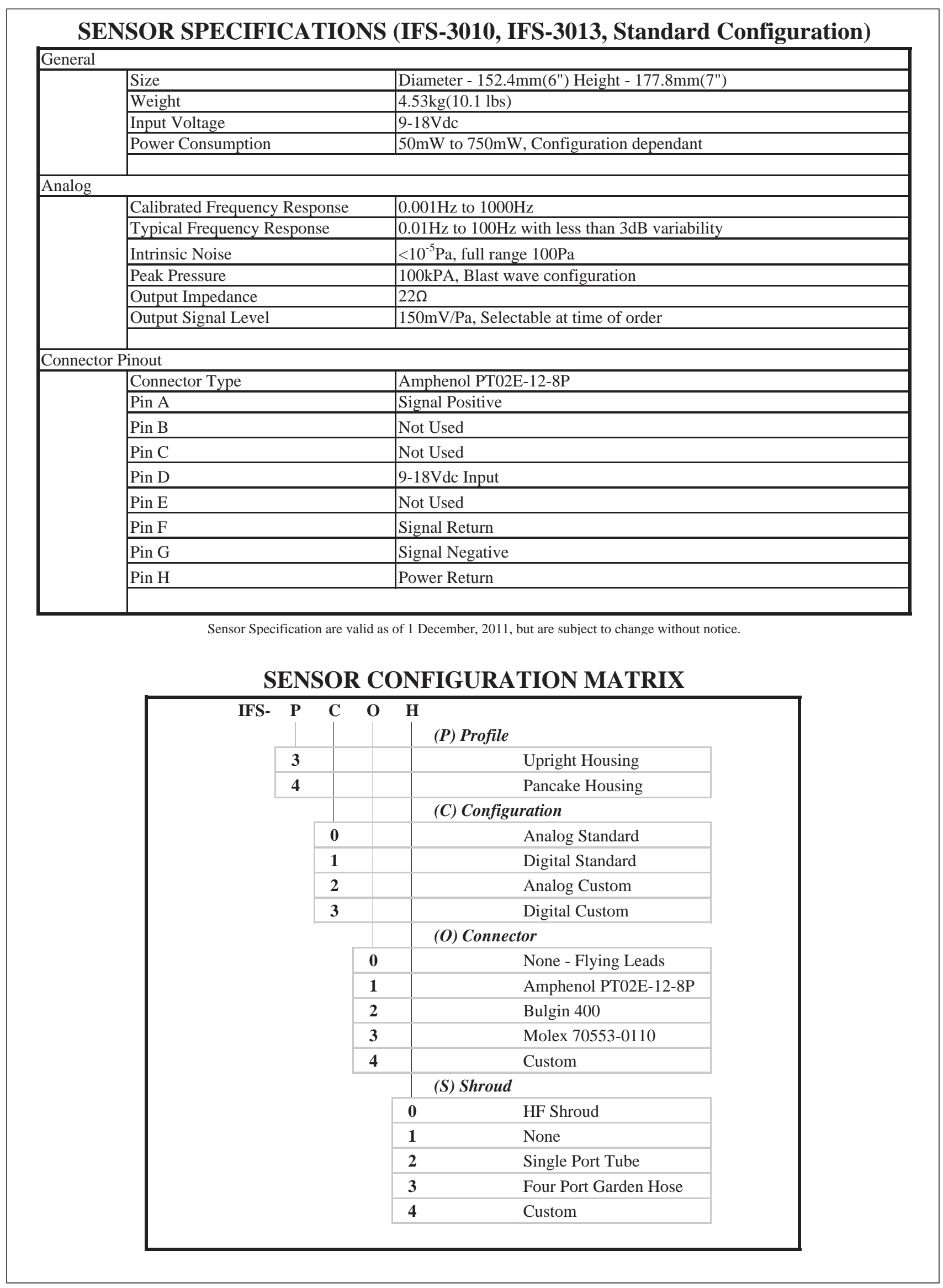

PAGE A-3

Final Report | DOE Award No. DE-EE0003269 


\section{Appendix B}

Shields' Report on Piñon Flat Data Analysis

PAGE B-1

Final Report | DOE Award No. DE-EE0003269 


\section{THE DETERMINATION OF WIND SPEEDS ALOFT FROM PRESSURE VARIATIONS ON THE GROUND}

Introduction

The determination of wind speeds from the pattern of pressure variations on the ground involves a modified version of the Taylor hypothesis. This hypothesis assumes atmospheric turbulence consists of a slowly changing pattern of eddies of various sizes that propagate with a constant diffusion or wind velocity. In the modified Taylor hypothesis, the different size eddies propagate with different velocities. The Fourier analysis of the pressure signals on the ground associates a particular frequency component with a particular size eddy moving across the pressure sensor with a particular speed. The size of the eddy is then associated with a wavelength in the pressure pattern. The wind velocity as a function of frequency is then interpreted as a measure of wind velocity as a function of eddy size. This research investigates the possibility of associating the eddy size with a height above the ground and thereby determining the wind velocity as a function of altitude. From very limited data Priestley estimates that the wavelength should be about 5 times the height for conditions of limited solar radiation of the ground. ( $\mathrm{J}$. T. Priestley, "Correlation studies of pressure fluctuations on the ground beneath a turbulent boundary layer", National Bureau of Standards Report No. 8942 (1965); also M. S. Thesis, University of Maryland (1965).)

The pressure pattern is determined from the response of an array of infrasound sensors. The wind velocity can be obtained by dividing the distance between sensors by the time shift necessary to give a maximum correlation between the sensor signals when the sensor separation is in the direction of the wind. This method for the velocity measurement is illustrated in Figures 1 and 2. Figure 1 shows the correlation for 10 sensor separation distances as a function of lag time. These particular curves are for a frequency of $0.127 \mathrm{~Hz}$. The correlation between all sensors separated by the same distance have been averaged to give a single correlation curve for that distance. Fig. 2 is a plot of the shift in lag time at which the peaks occur verses the sensor separation. The experimental points are fitted with a least squares straight line. The inverse slope of this line is taken as the wind speed at this frequency.

Problems can arise when the wind carries the pattern of turbulence across the line of sensors at an angle. One might assume that the wind direction could be obtained from the velocity measured as in Fig. 2 in two directions. However this would be possible only if the same turbulence pattern crosses all of the sensors. A failure of this condition is indicated when the data plotted as in Fig.2 does not fall on a straight line. 
The infrasound arrays and data analyzed

Three different arrays have been used in acquiring the data here analyzed. These data were originally acquired in the study of the use of arrays to separating infrasound from wind noise. The first and original array is described in the 2005 paper by Shields. (F. Douglas Shields, "Low frequency wind noise correlation in microphone arrays", J. Acoust. Soc. Am. 117 (6), 3489-3496 (2005)). It consisted of 28 sensors arranged in rectangular arms. The horizontal arms were on the ground and were oriented parallel and perpendicular to the wind direction. An ultrasonic anemometer was mounted on a pole $3 \mathrm{~m}$ above the ground a few meters from the corner of the array. Data collected using this array from three different sites have been analyzed. There were several hundred meters to any obstruction of the wind at all three sites. Figure 2 of the cited paper shows a photograph of the array deployed at one of the sites which was a sod field.

The second array contained 100 sensors similar to those described in the cited paper, but of improved design and with a broader frequency response. The one hundred sensors were arranged in a square ten sensors on an edge and $2 \mathrm{~m}$ apart. In this case, the ultrasonic anemometer was mounted 2.5 meters off the ground close to the array. This array was deployed at the International Test Band Infrasound monitoring station at Piñon Flat, CA. The data analyzed here were recorded in September and October of 2007. A photograph of this site is shown in Fig. 3.

The third array consisted of 96 sensors similar in design to those in the second array. However, in this case the sensors were arranged in radial arms 30 degrees apart with 16 sensors in each arm. The sensors were spaced 4 meters apart in the arms with either a $12 \mathrm{~m}$ or a $16 \mathrm{~m}$ spacing in the center, giving an aperture varying for alternate arms from 68 or $72 \mathrm{~m}$. This array was also deployed at Piñon Flat but in July of 2008.

Analysis of the data

The Piñon Flat data taken with the square array have been examined first. Samples of data have been chosen for three different wind velocities and for day and night time to see if the frequency dependence of the velocity can be associated with a particular time of day, wind direction, or wind velocity. The correlation of 10 minute samples of data have been calculated for lag times of approximately one period using the Labview xcov program.

The velocities obtained as a function of frequency from four such 10 minute sample periods with approximately the same average wind velocity have been plotted in Figs $4-9$. Also shown in each figure is the average of the four samples. The average velocity as measured by the anemometer which is $2.5 \mathrm{~m}$ above the ground is not a function of frequency but it has been plotted as a red cross on the 
figures at a frequency of $0.12 \mathrm{~Hz}$. Figures 4-9 show that the variation of velocity with frequency varies erratically with frequency and time. There is a similar variation in the direction of the wind. It is particularly difficult to understand how the velocity can decrease at low frequencies in figures 6,8 and 9.

In Fig. 10, the average velocity curves in Figures 4 through 9 have been divided by the frequency and the log of the resulting wavelengths plotted against the log of the velocity. The different curves have been labeled with the corresponding anemometer velocities. A visual examination shows that for all of the curves the frequency dependent wind velocities corresponding to the anemometer occur for a wavelength of approximately $10 \mathrm{~m}$. Since the anemometer was approximately 2 meters above the ground, this is in fair agreement with Priestley's estimate that the eddy size was approximately 5 times the height of the above the ground. Furthermore, all of the velocities increase at a rate of approximately $1 / 40^{\text {th }}$ the rate of increase in the wavelength. As an illustration, the eddy size a height of $80 \mathrm{~m}$ would be about 400 meters and the wind velocity at that altitude about twice the value measured at a height of $2 \mathrm{~m}$.

As a further check on these conclusions, measurements made with the two other arrays discussed in the introduction have been analyzed. Figs. 11 and 12 show results of measurements made with the radial array at Piñon Flat in July of 2008. The velocity verses frequency for four different wind velocities have been plotted in Fig. 11, and the wavelengths verses wind velocities plotted in Fig. 12. As in Fig. 10, the curves are label with the anemometer velocities. From a comparison between the curves in Figures 10 and 12, the 40 to 1 rate of increase in the wavelength with wind velocity is roughly the same. However, the anemometer velocities measured again at $2 \mathrm{~m}$ above the ground correspond to wavelengths of about $5 \mathrm{~m}$ in Fig. 12 compared to $10 \mathrm{~m}$ in Fig. 10.

The results of the measurement reported 2005 that were made with the 28 sensor, three dimensional array are plotted in Figures 13 and 14. These measurements were made at three different sites. This time the anemometer was 3 $\mathrm{m}$ above the ground. At all three of the sites the wind velocity as measured by the anemometer was approximately $6 \mathrm{~m} / \mathrm{s}$. As read from the plots in Fig. 14, this anemometer velocity would correspond to a wavelength of approximately $100 \mathrm{~m}$ or about 10 times that of the Piñon Flat value. The rate of increase in wavelength with velocity is about $1 / 2$ that observed for the Piñon Flat data.

\section{Conclusions}

Wind noise measurements at ground level indicate a frequency dependent wind velocity which has been interpreted as a dependence of the wind velocity upon wavelength or eddy size. Though varying considerably with frequency and time, a general increase in velocity with eddy size has been observed. However, 
further investigation is necessary to determine how this relationship depends upon atmospheric conditions and terrain.

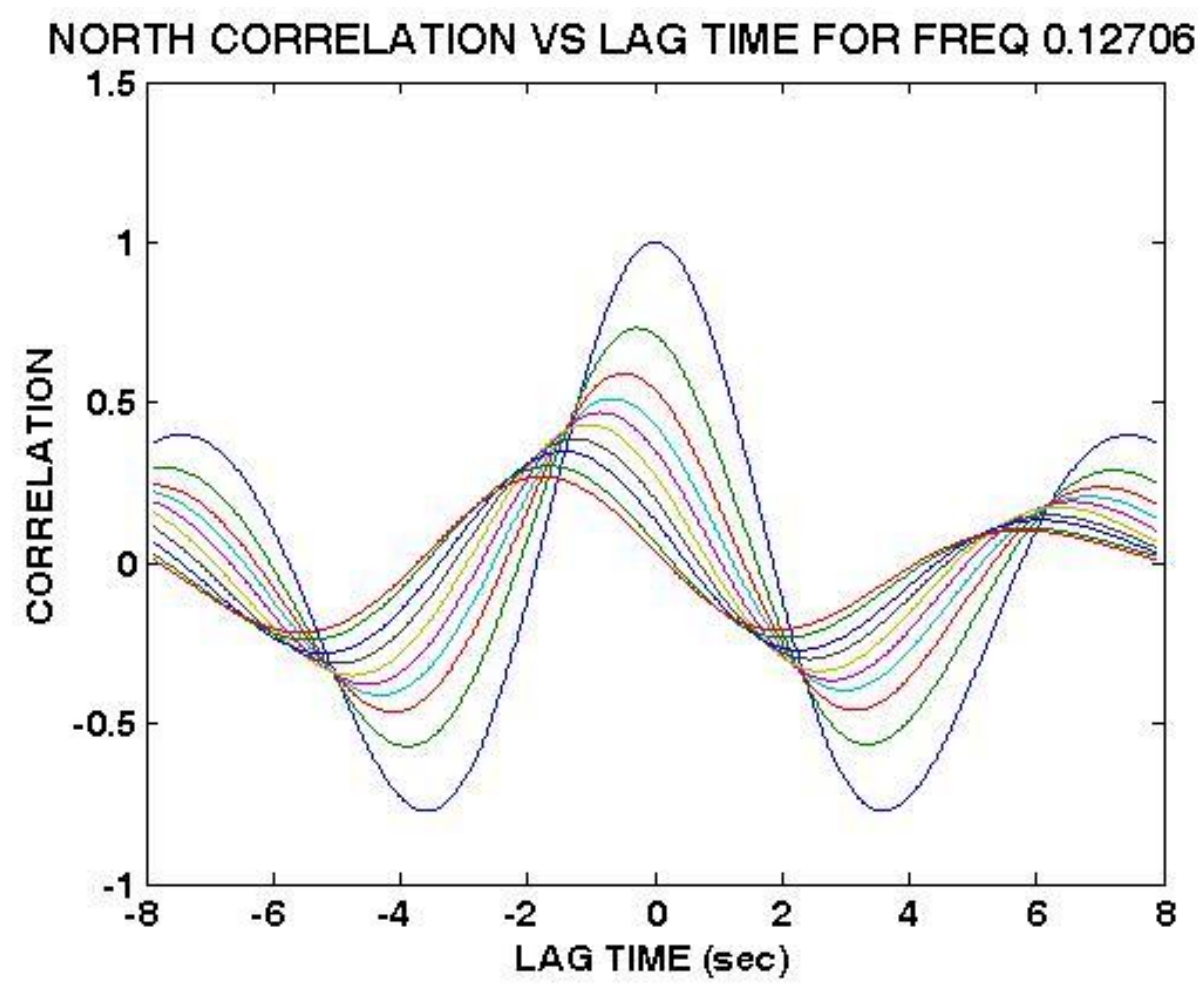

Fig 1. Correlation between signals from the 100 sensor square array. The signals have been filtered with a one third octave filter with a center frequency of 0.127 Hz. The curves shown are the average curves for correlations between all sensors with the same separation in the north south direction. The measurements were made on Oct. $17^{\text {th }}$ at 10 A. M. The wind velocity was about $8.5 \mathrm{~m} / \mathrm{s}$ from the south. 


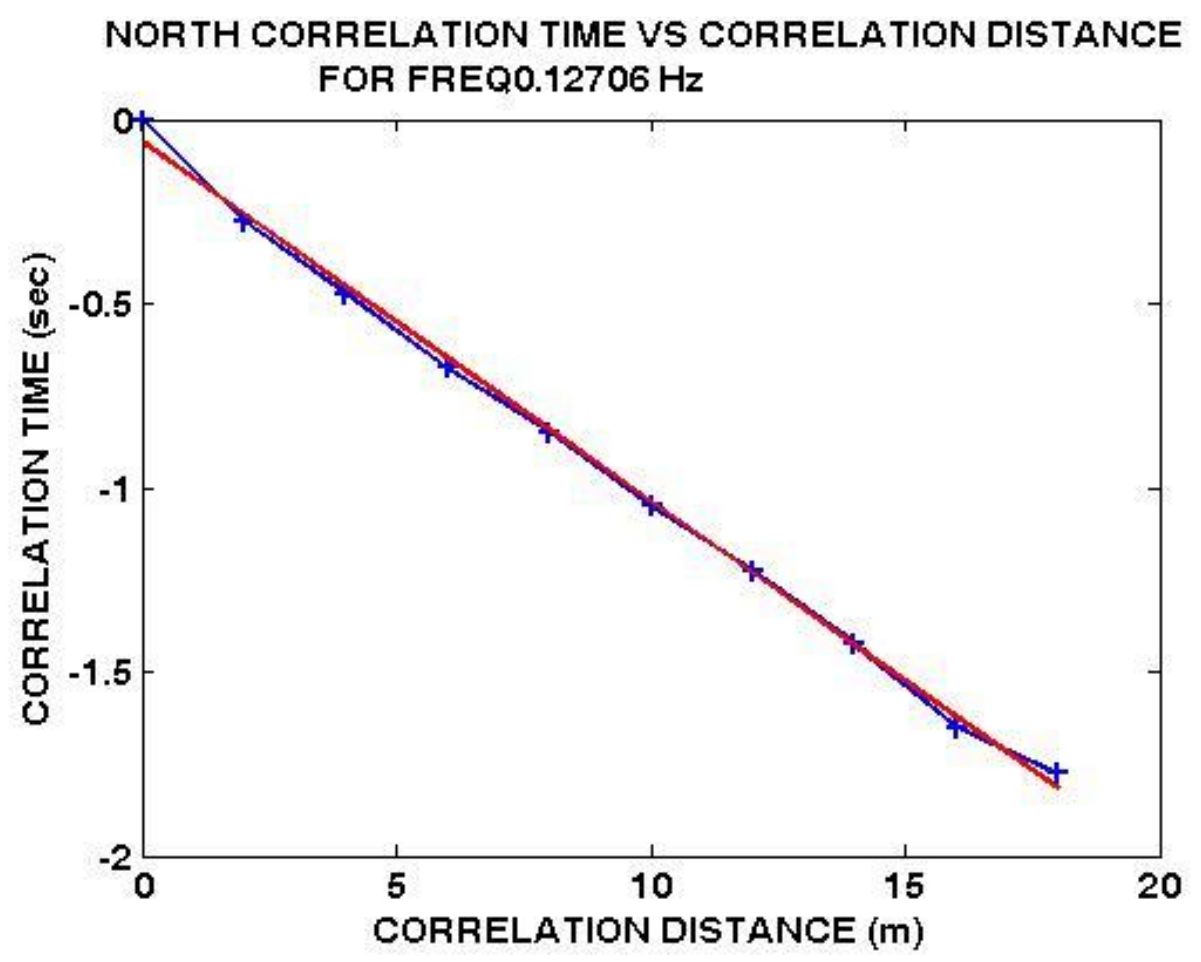

Fig. 2 Lag time for correlation peaks in Fig. 1 verses sensor separation. The red curve is a least squares fit to the measured points in blue. The wind velocity is the reciprocal of the slope. 


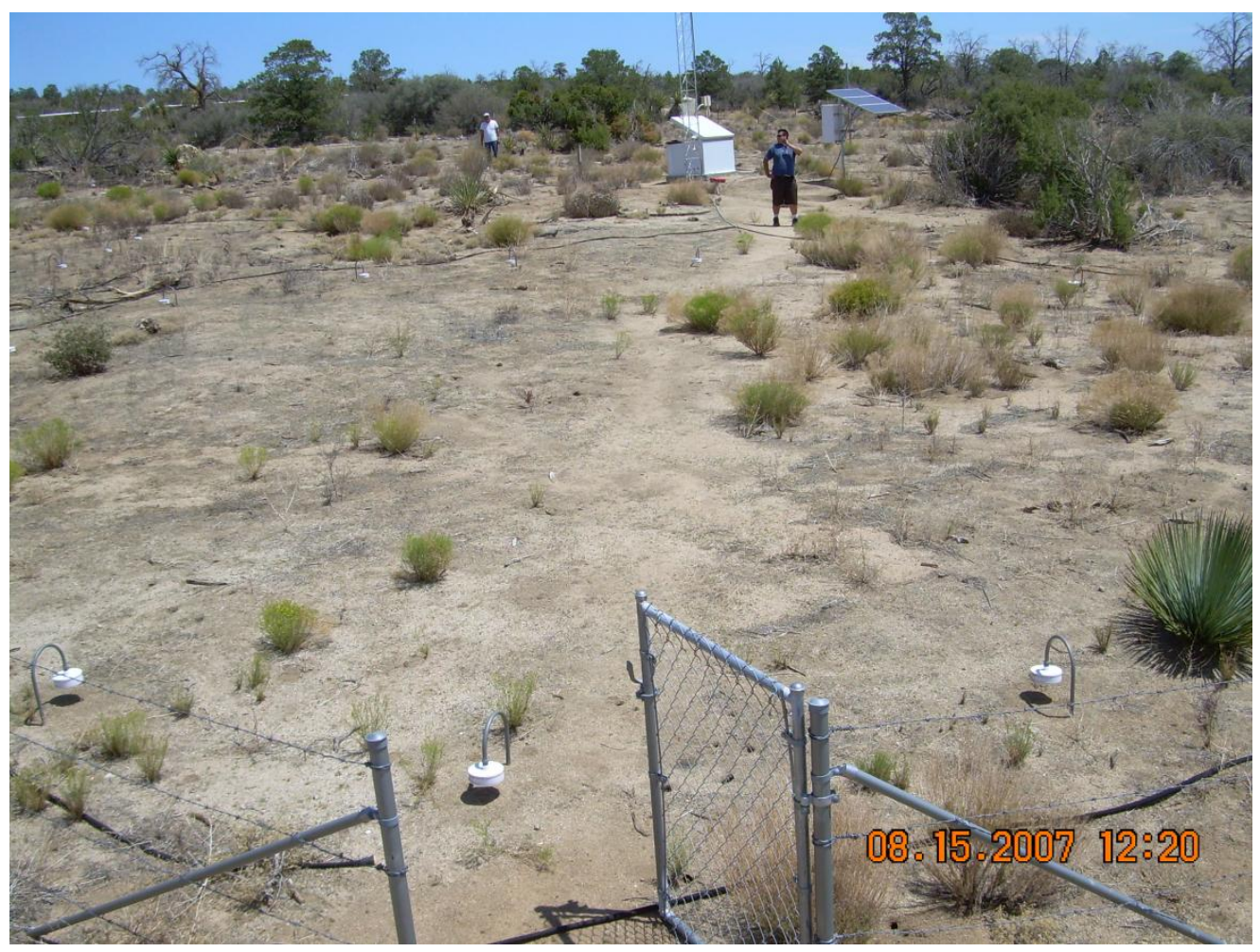

Fig. 3 The site at Piñon Flat, CA where the measurements were made. 


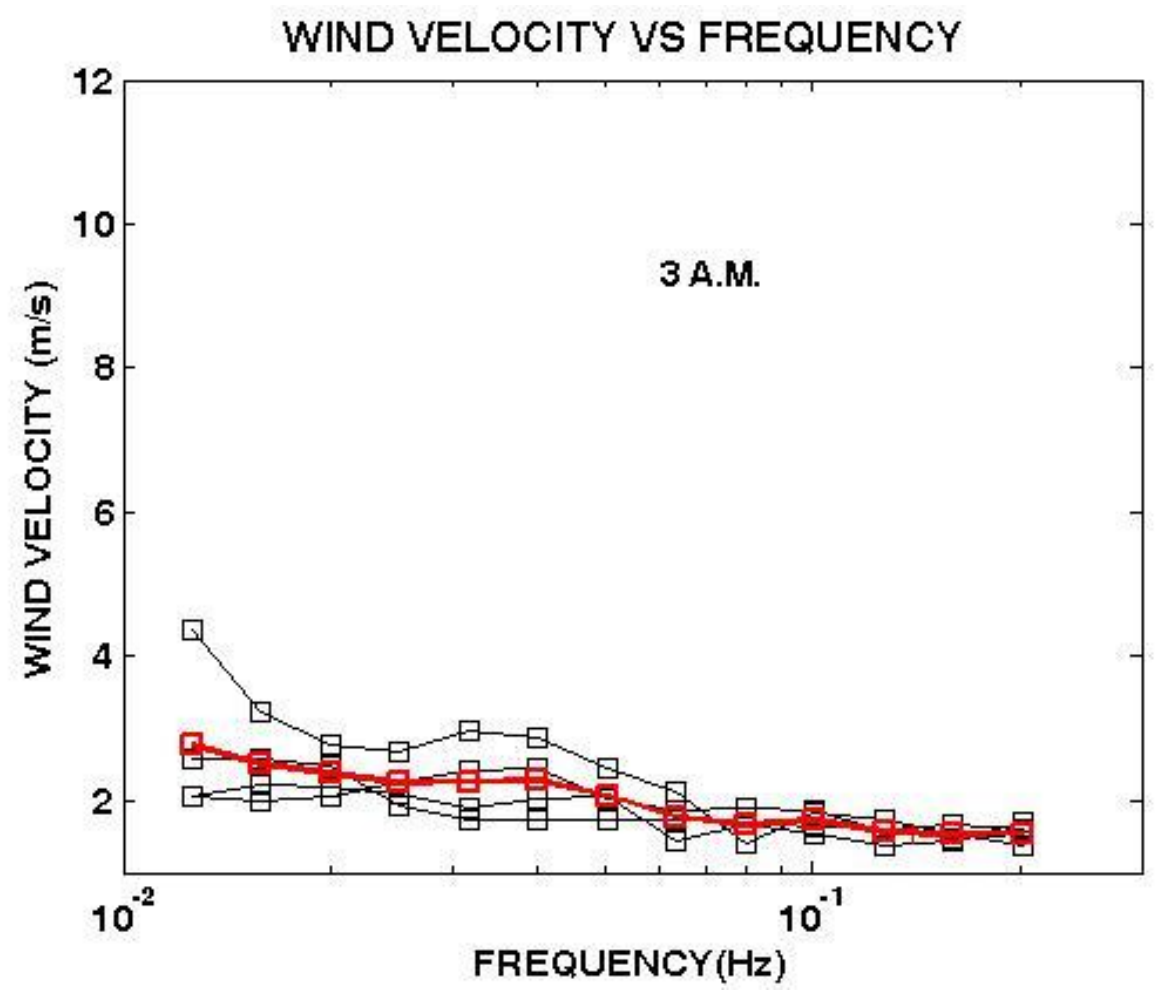

Fig. 4. Wind velocity vs frequency for Sept. 12, 2007 at 3 A.M. The average velocity as measured by the anemometer was about $1.5 \mathrm{~m} / \mathrm{s}$. The blue curves are four individual 10 minute runs. The red curve is the average of the four individual runs. 


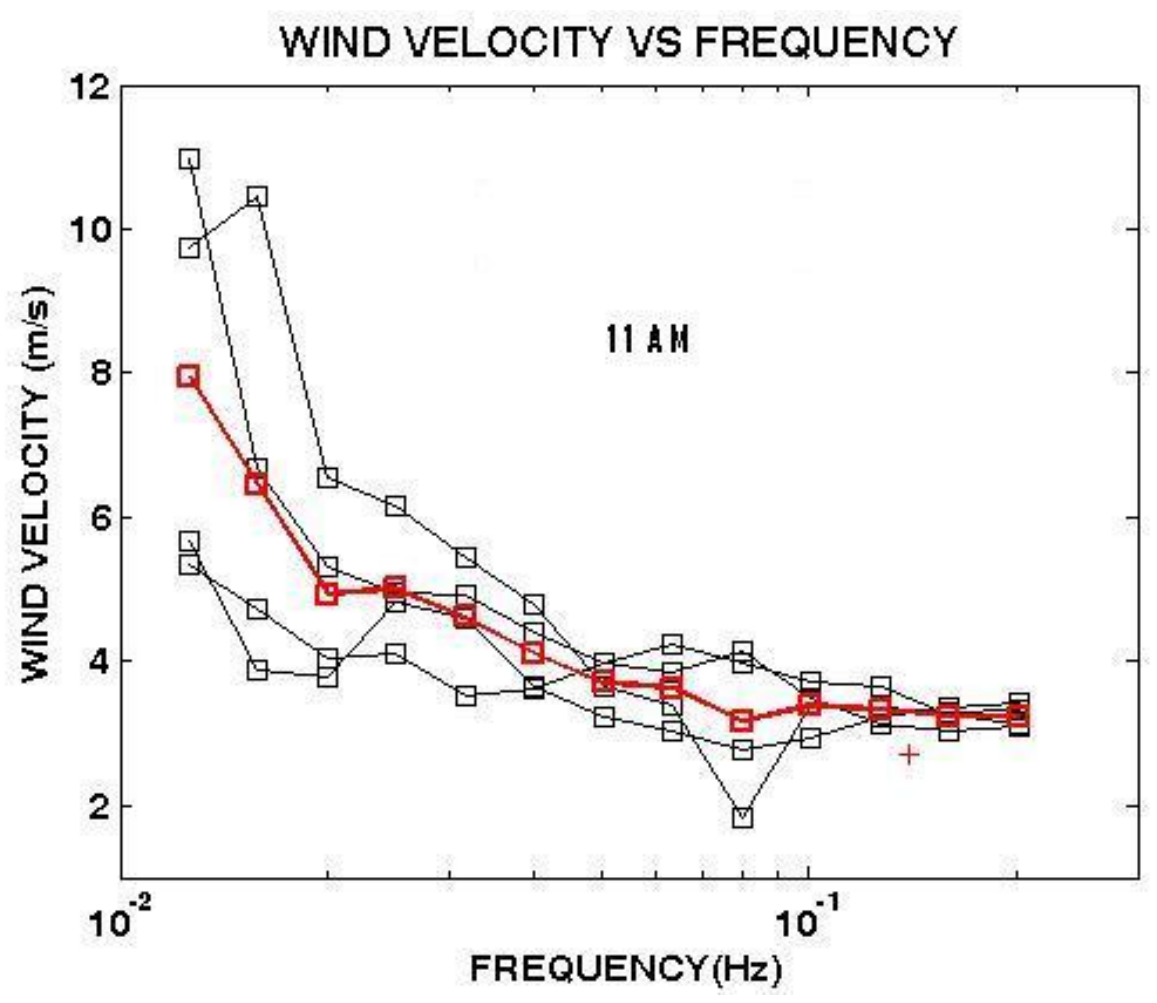

Fig. 5 Wind velocity vs frequency for Sept. 27 at 11 A.M. The average wind velocity as measured by the anemometer is about $2.7 \mathrm{~m} / \mathrm{s}$ and is plotted as the red cross arbitrarily at $0.12 \mathrm{~Hz}$. The blue curves are four individual 10 minute runs. The red curve is the average of the four individual runs. 


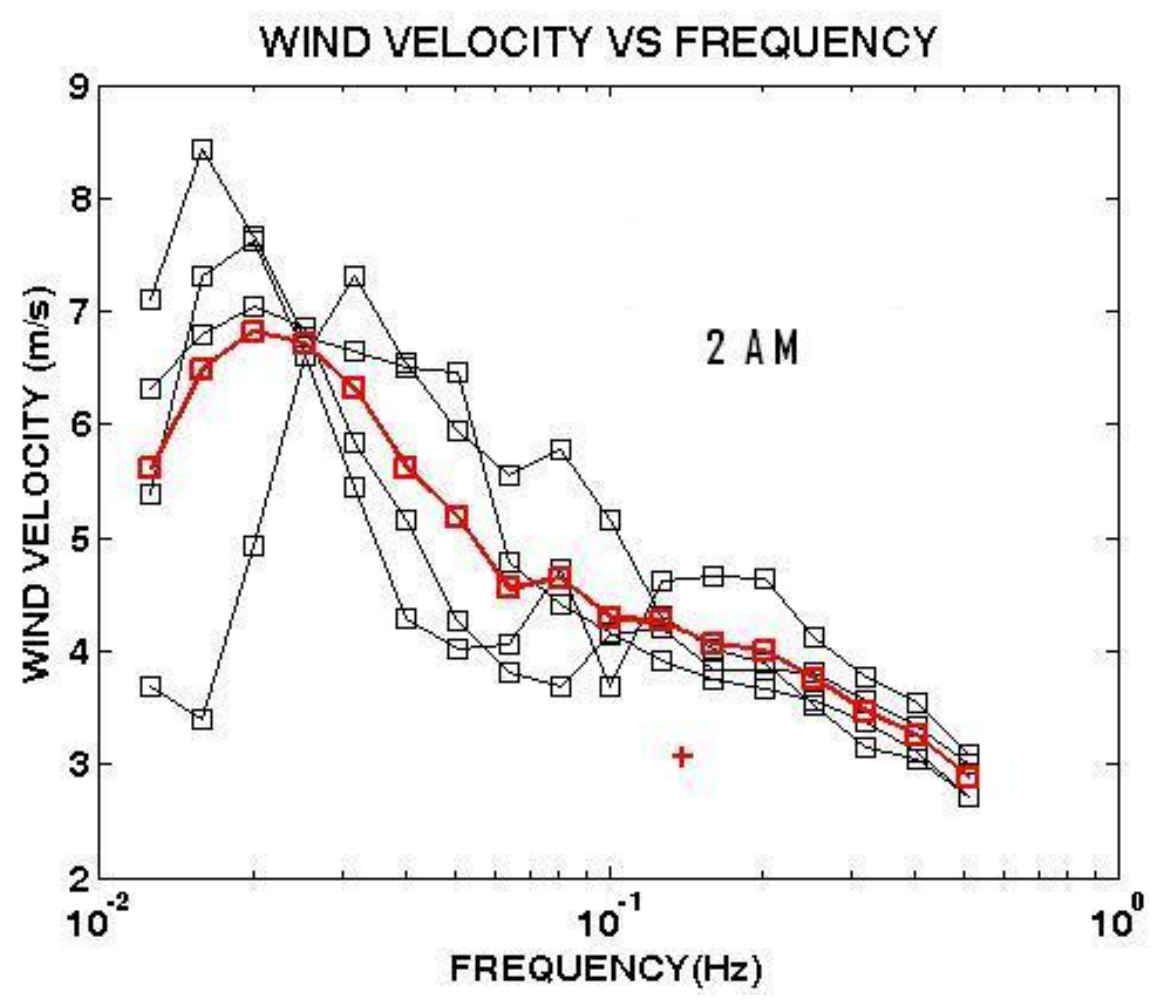

Fig. 6 Wind velocity vs frequency for Oct. 7 at 2 A.M. The average wind velocity as measured by the anemometer is about $3.1 \mathrm{~m} / \mathrm{s}$ and is plotted as the red cross arbitrarily at $0.12 \mathrm{~Hz}$. The blue curves are four individual 10 minute runs. The red curve is the average of the four individual runs. 


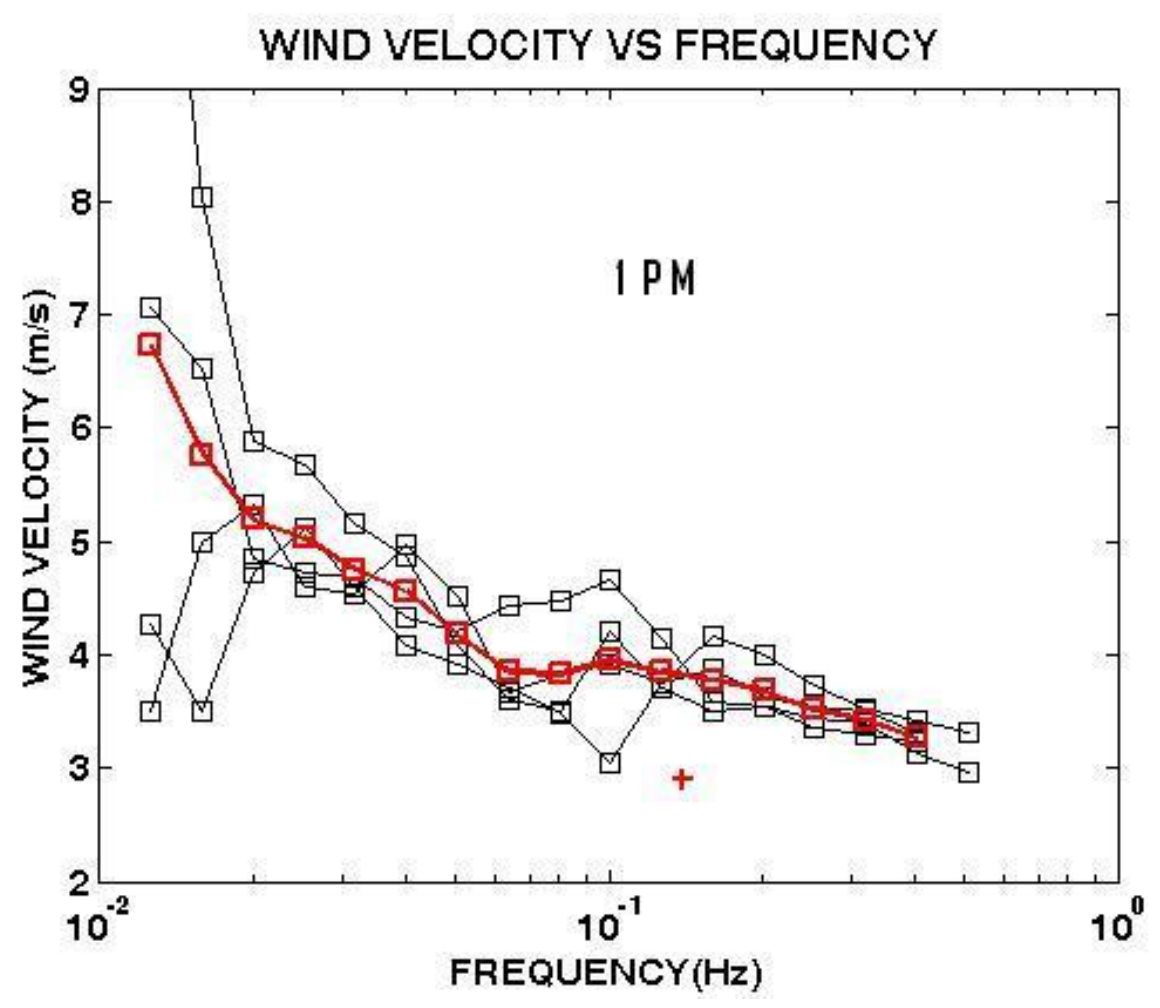

Fig. 7 Wind velocity vs frequency for Sept. 18 at 1 P.M. The average wind velocity as measured by the anemometer is about $2.9 \mathrm{~m} / \mathrm{s}$ and is plotted as the red cross arbitrarily at $0.12 \mathrm{~Hz}$. The blue curves are four individual 10 minute runs. The red curve is the average of the four individual runs. 


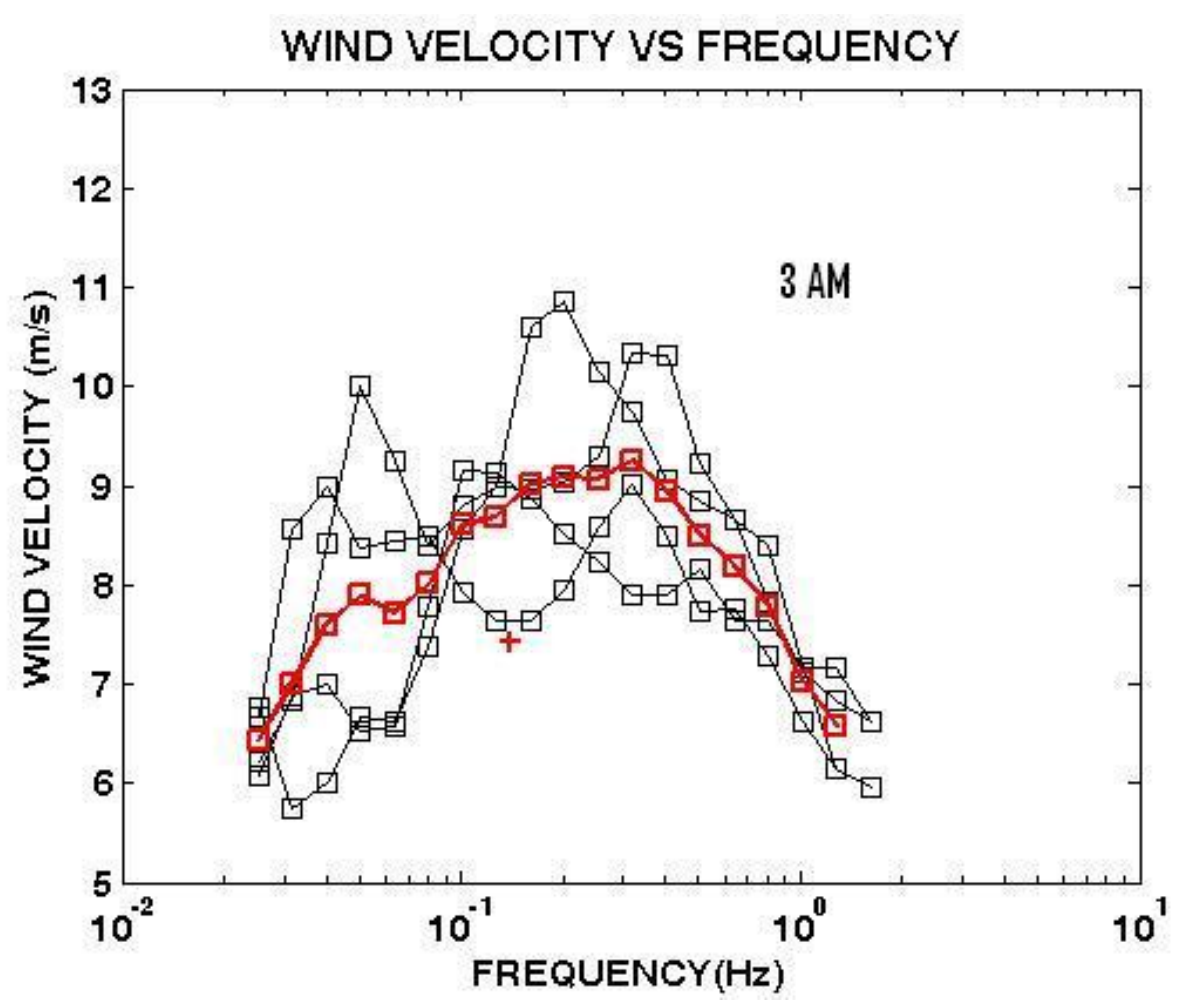

Fig. 8 Wind velocity vs frequency for Sept. 29 at 3 A.M. The average wind velocity as measured by the anemometer is about $7.4 \mathrm{~m} / \mathrm{s}$ and is plotted as the red cross arbitrarily at $0.12 \mathrm{~Hz}$. The blue curves are four individual 10 minute runs. The red curve is the average of the four individual runs. 


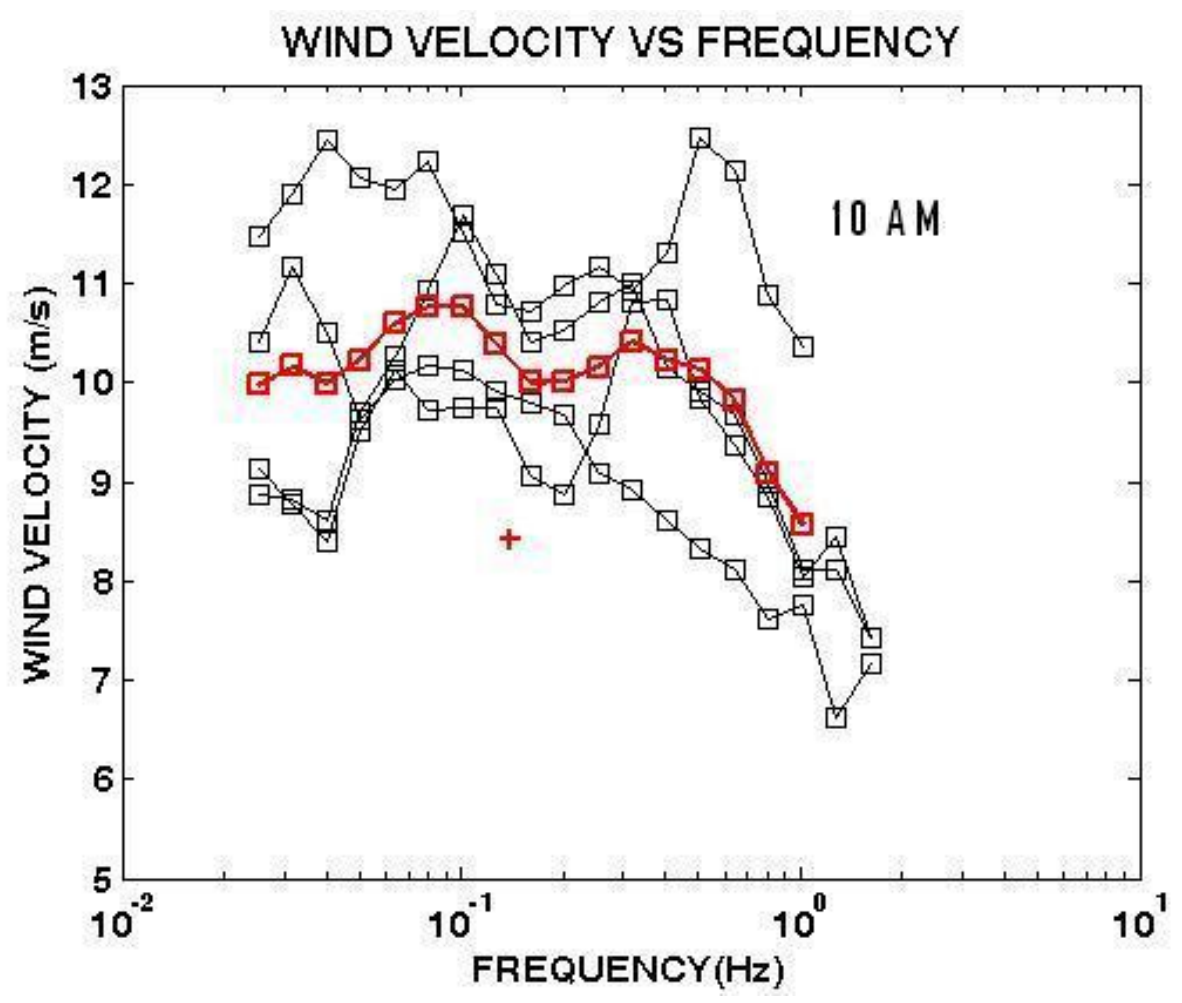

Fig. 9 Wind velocity vs frequency for Oct. 17 at 10 A.M. The average wind velocity as measured by the anemometer is about $8.4 \mathrm{~m} / \mathrm{s}$ and is plotted as the red cross arbitrarily at $0.12 \mathrm{~Hz}$. The blue curves are four individual 10 minute runs. The red curve is the average of the four individual runs. 


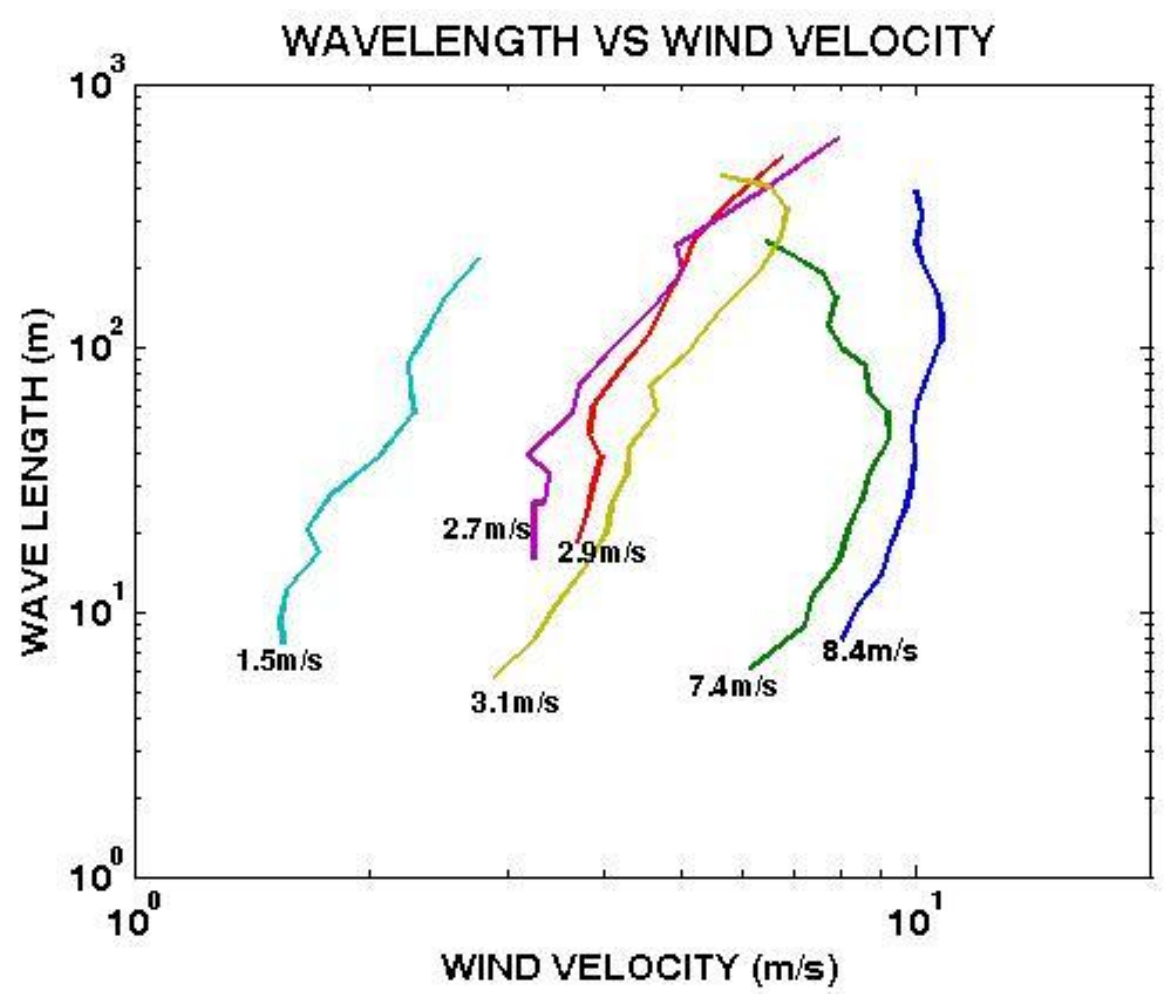

Figure 10 Wavelengths verses wind velocity for data taken with the 100 sensor square array in Sept. and Oct. of 2007. Each curve is the average of four ten minute runs at approximately the same wind velocity. The curves are labeled with the average velocity as read by an ultrasonic anemometer about 2 meters above the ground. 


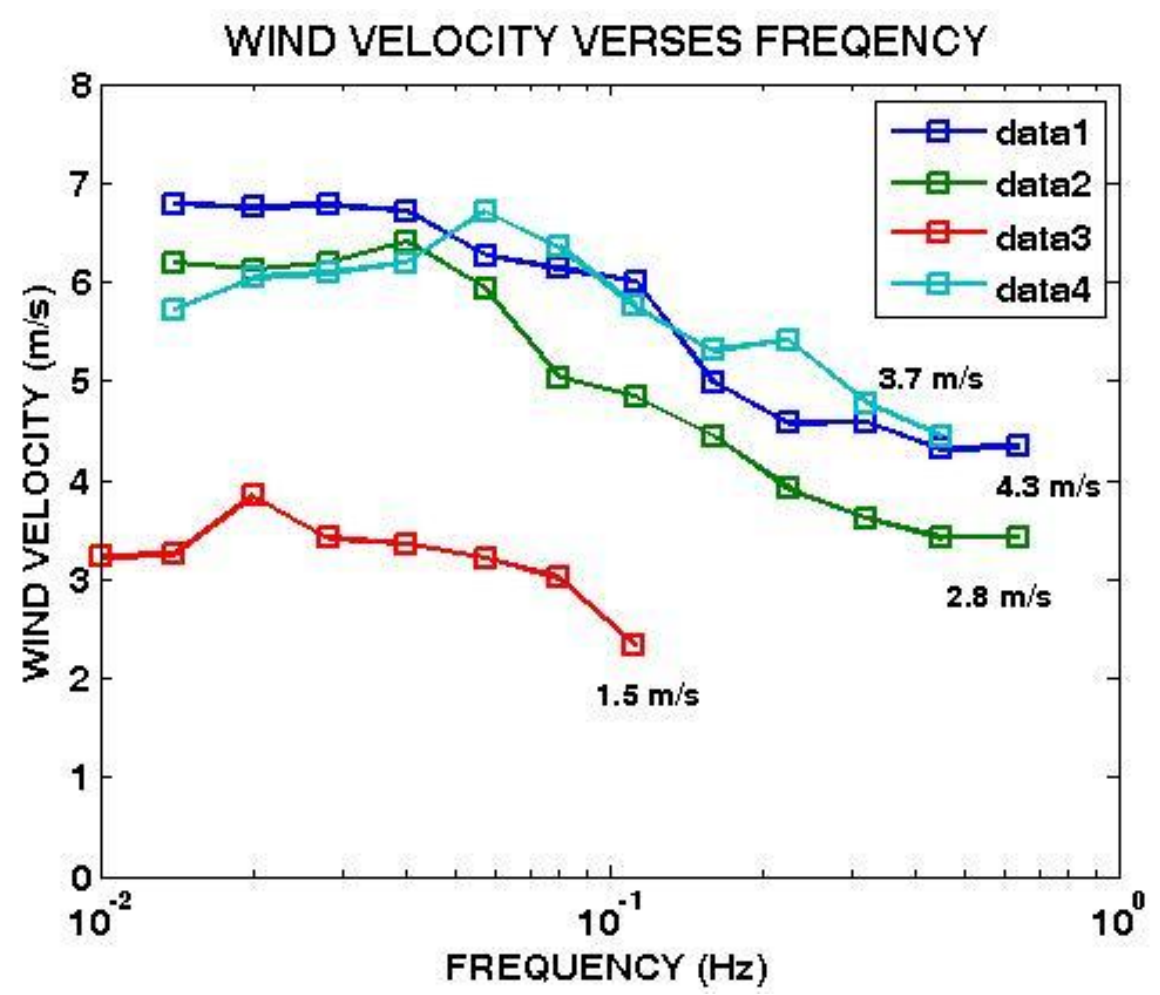

Figure 11. Wind velocity verses frequency for data take with the 96 sensor radial array at Piñon Flat, CA in July of 2008. The curves are labeled with velocities measured with an ultrasonic anemometer 2 meters above the ground. 


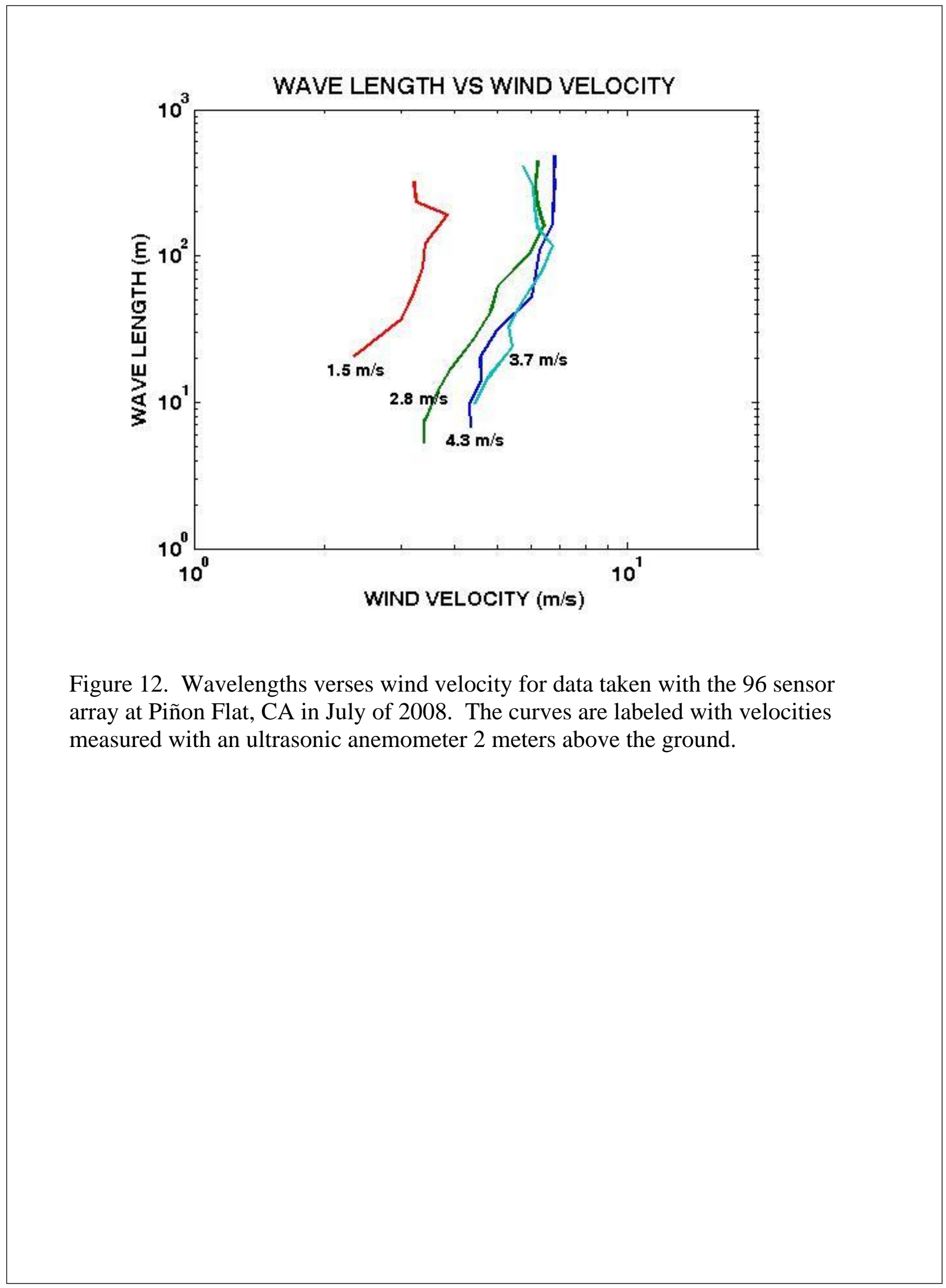




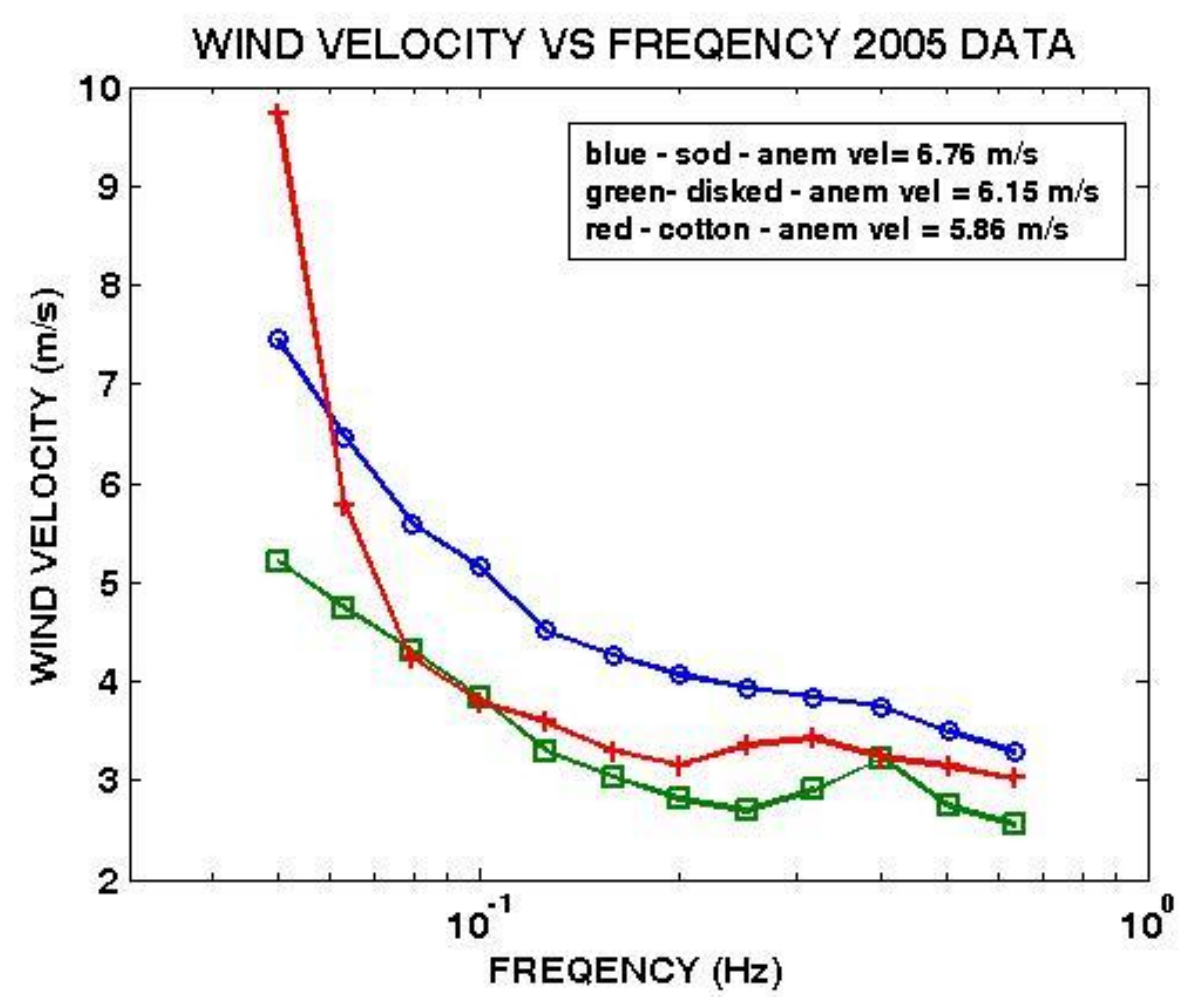

Figure 13. Wind velocity verses frequency for measurements made in 2005 with the 28 sensor array at three different sites. Velocities were obtained from correlation lag times between 10 sensors in the arm parallel to the wind direction. 


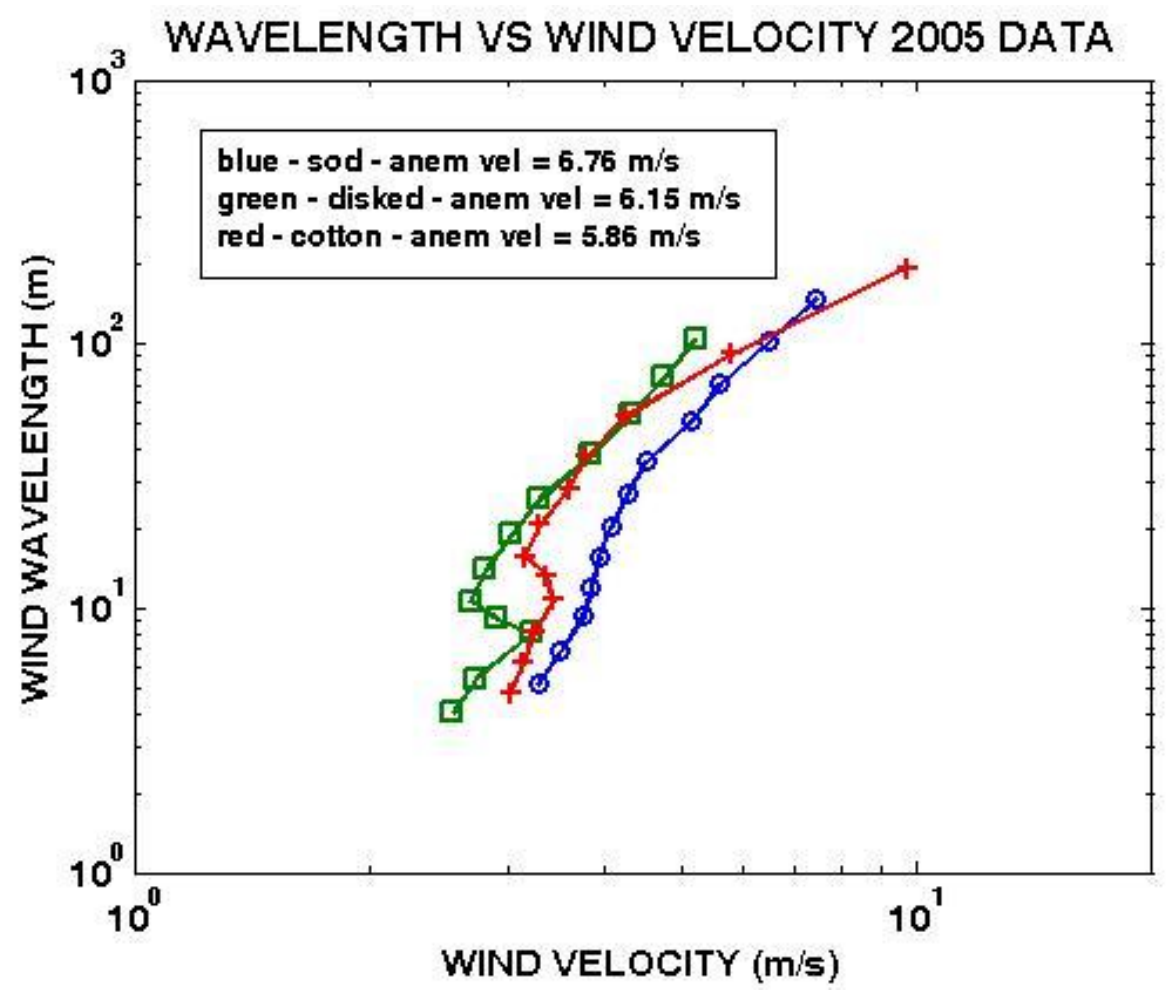

Figure 14. Wind wavelength verses wind velocity for measurements made in 2005 with the 28 sensor array at three different sites. Velocities were obtained from correlation lag times between sensors in the arm parallel to the wind direction. 


\section{Appendix C}

Combustion Research \& Flow Technology - Final Report

PAGE C-1

Final Report | DOE Award No. DE-EE0003269 


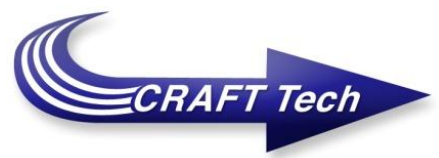

Combustion Research and Flow Technology, Inc.

6210 Keller's Church Road, Pipersville, PA 18947

Phone: 215/766-1520 Fax: 215/766-1524

CRAFT Tech Report No. CRAFTR-08.2011.030

C459

\section{Passive Acoustic Detection of Wind}

Turbine In-Flow Conditions for Active

Control and Optimization

\section{Final Report}

Prepared by

Combustion Research and Flow Technology, Inc.

6210 Keller's Church Rd.

Pipersville, PA 18947

Attn: Neeraj Sinha

Phone: 215-766-1520

E-mail: sinha@craft-tech.com

\section{August 2011}

Prepared for

University of Mississippi

National Center for Physical Acoustics

Coliseum Drive

University, MS 38677

Attn: Dr. Nathan Murray

Phone: 662-915-3190

E-mail: nmurray@olemiss.edu

Prepared under

Prime Contract Number: Part I Final DE-EE0003269

UM Subcontract No. 11-09-22

UM Acct. No. 300212414A

Period of Performance: 01 May 2010 to 31 August 2011 
Appendix C | Combustion Research \& Flow Technology - Final Report

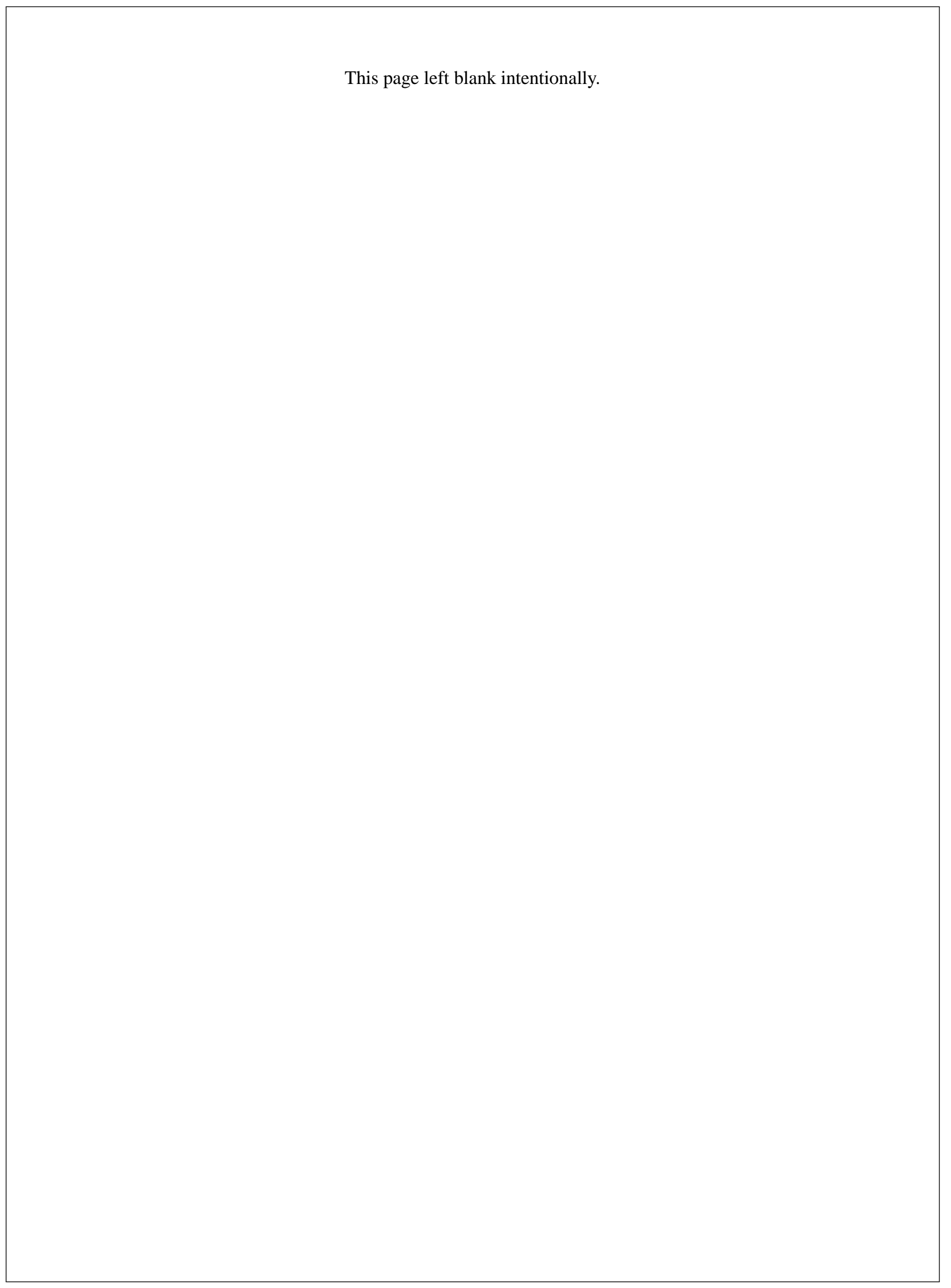

PAGe C-3

Final Report | DOE Award No. DE-EE0003269 


\section{TABLE OF CONTENTS}

CHAPTER 1 INTRODUCTION

CHAPTER 2 LARGE-EDDY SIMULATION OF ATMOSPHERIC BOUNDARY LAYER.........2

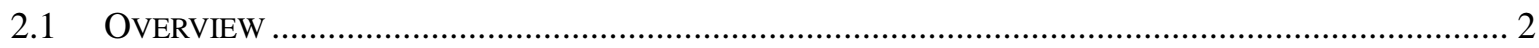

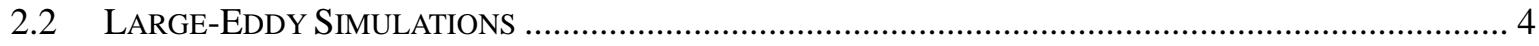

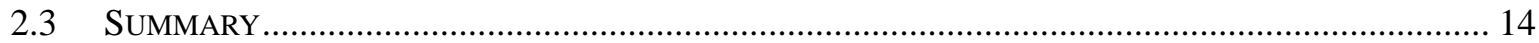

CHAPTER 3 DESIGN OPTIMIZATION OF WIND TURBINE BLADES ................................15

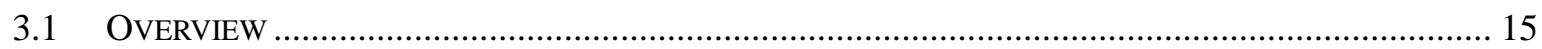

3.2 SUMMARY OF BASELINE BSDS DESIGN UTILIZED FOR CURRENT STUDY ................................. 16

3.3 AERODYNAMIC AND AEROACOUSTIC SiMULATION OF BASELINE BSDS BLADE ....................... 18

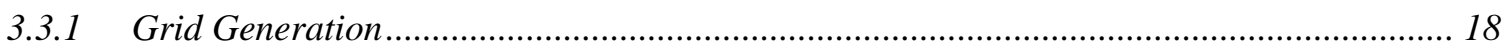

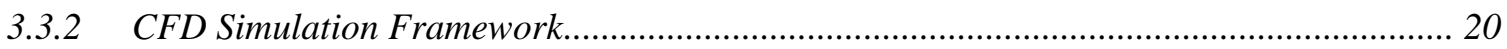

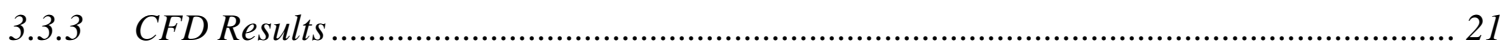

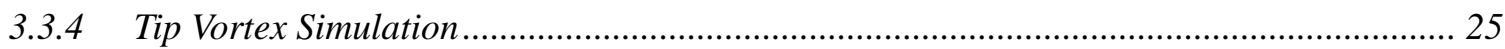

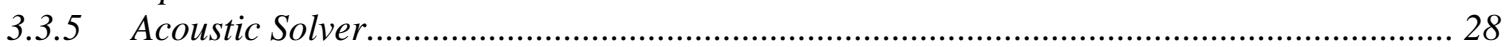

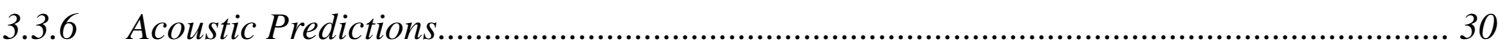

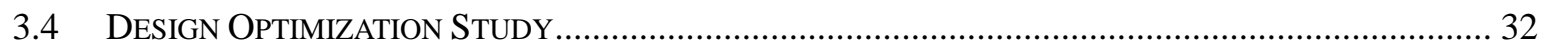

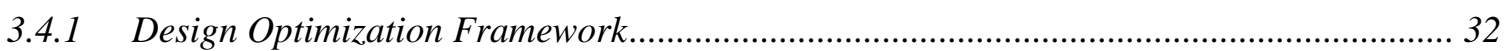

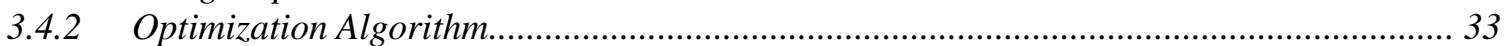

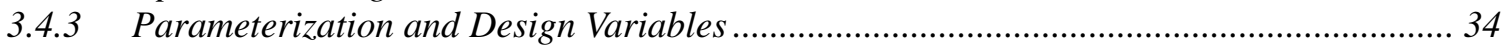

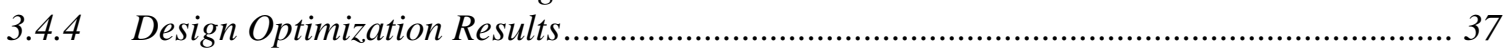

3.4.5 Summary of Optimization Studies ...................................................................................... 44

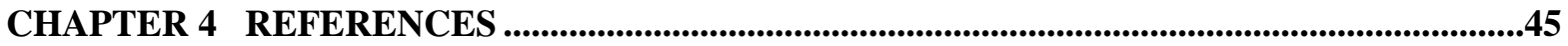

Final Report

iii

CRAFTR-08.2011.030

Prime Contract No. DOE-DE-EE0003269

Combustion Research and Flow Technology, Inc. 
Figure No.

\section{List of Figures}

FIGURE 1: EXPERIMENTAL SETUP OF THE BACKWARD-FACING STEP CONFIGURATION IN THE SUBSONIC WIND TUNNEL. 2

FigURE 2: MEAN STREAMWISE VELOCITY, U IN THE MID-SPAN PLANE OF THE WIND TUNNEL.................... 3

FIGURE 3: STREAMWISE VELOCITY, U PROFILES (A) SHOWING THE SEPARATED REGION, AND (B) THE BOUNDARY LAYER PROFILE.

FigURE 4: MEAN CONTOURS OF THE MACH NUMBER IN THE MID-SPAN PLANE AND THE BOTTOM WALL FOR THE 3D LES.

FIGURE 5: MEAN STREAMWISE VELOCITY, U CONTOURS FOR THE 2D LES AT FOUR REPRESENTATIVE INSTANTS IN TIME SHOWING THE DEVELOPMENT OF THE EDDIES/GUSTS IN THE BOUNDARY LAYER DOWNSTREAM OF THE STEP.

FIGURE 6: SCHEMATIC OF THE GRID AS CUT THROUGH THE MID-SPAN OF THE COMPUTATIONAL DOMAIN USED FOR THE CFD SIMULATIONS.

FiguRE 7: INSTANTANEOUS SNAPSHOTS OF THE MACH NUMBER CONTOURS ON THE MiD-SPAN PlaNE OF THE SIMULATION AT FOUR INSTANTS IN TIME SHOWING THE UNSTEADY DEVELOPMENT OF THE BOUNDARY LAYER ON THE BOTTOM WALL.

FiguRE 8: INSTANTANEOUS SNAPSHOTS OF THE MACH NUMBER CONTOURS ON THE MID-SPAN PlaNE OF THE SiMULATION ALONG WITH CROSS-STREAM CONTOURS OF W-VELOCITY AT X=5, 12, 20" FROM THE STEP SHOWING THE UNSTEADY DEVELOPMENT OF THE BOUNDARY LAYER ON THE BOTTOM WALL.... 9

Figure 9: MeAn Streamwise Velocity Distribution Close to the Wall RePRESENTATIVE OF the SEPARATED FLOW REGION IN THE FLOW FIELD.

Figure 10: Mean Streamwise Velocity Distribution along the Wall Normal Direction(Y) at a FEW STREAMWISE STATIONS (FOR THE ADIABATIC WALL BOUNDARY LAYER).

Figure 11: MEAN Streamwise Velocity Distribution along the Wall Normal Direction at a FEW STREAMWISE STATIONS (FOR THE ADIABATIC WALL BOUNDARY LAYER).

Figure 12: MEAN TEMPERATURE Distribution ALONG the WALl Normal DiRECTION AT A FEW

STREAMWISE STATIONS (FOR THE ISOTHERMAL WALL BOUNDARY LAYER).

FIGURE 13: BOUNDARY LAYER PROFILE AT THE END OF THE 8FT SECTION OF THE WIND TUNNEL............. 12

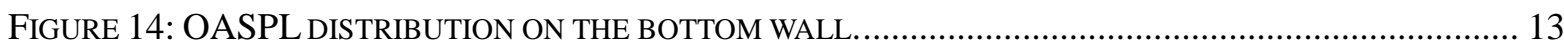

FigURE 15: SPL SPECTRA ON THE BOTTOM WALL FOR THE AdiABATIC WALL COMPARED WITH AVAILABLE

EXPERIMENTAL DATA AT LOWER SPEEDS.

FIGURE 16: SPL SPECTRA ON THE BOTTOM WALL COMPARING THE ADIABATIC AND ISOTHERMAL WALL CONDITIONS.

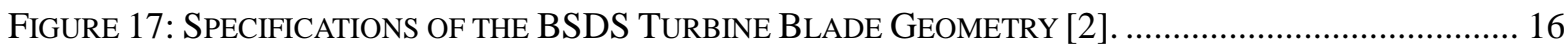

FIGURE 18: AIRFOIL SHAPES OF THE DIFFERENT SECTIONS OF THE BSDS TURBINE BLADE [2] .............. 17

FIGURE 19: BSDS SHAPE DESIGN SHOWING LOFT, TWIST AND SECTIONS [2] ...................................... 17

Figure 20: Multi-Element Mesh for One Blade Passage of Three-Bladed Wind Turbine. ..... 19

FIGURE 21: GRID TOPOLOGY NEAR THE HUB AND THE BLADE........................................................ 20

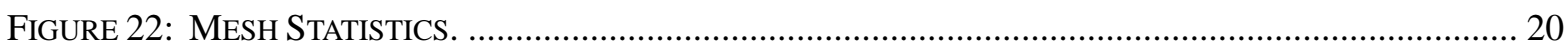

Figure 23: Pressure Distribution on THE Suction Side of BSDS Blade..................................... 22

Figure 24: Pressure Distribution ON THE Pressure Side OF BSDS Blade................................... 22

Final Report

iv

CRAFTR-08.2011.030

Prime Contract No. DOE-DE-EE0003269

Combustion Research and Flow Technology, Inc. 
Figure 25: Chordwise Pressure Distribution at 5 SPANwiSe LocAtions $(20 \%, 45 \%, 65 \%, 85 \%$ $95 \%)$

FiguRE 26: COMPARISON OF TURBINE PERFORMANCE FOR THE CFD COMPUTATION WITH DESIGN DATA

[2].

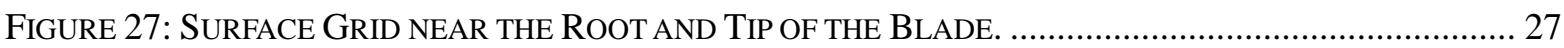

FIGURE 28: High RESOLUTION GRID TO RESOLVE THE TIP VORTEX. .................................................... 27

FIGURE 29: ISO-SURFACE OF VORTICITY SHOWING TIP VORTEX AND VORTICITY PRODUCTION AROUND THE

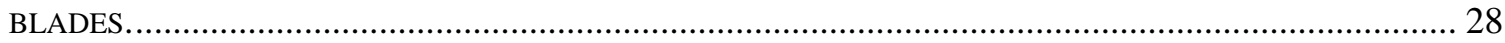

Figure 30: ACOUSTIC TOOLKIT USED TO PREDICT NOISE FROM CFD SOLUTIONS OF RotaTing MACHINERY.

Figure 31: SCHEMATIC OF FrAMEWORK FOR PREDICTING NOISE FROM WIND TURBINES INCLUDING GROUND EFFECTS.

FIGURE 32: SCHEMATIC SHOWING LOCATION OF MICROPHONES FOR PREDICTING NOISE FROM THE OPERATION OF WIND TURBINE.

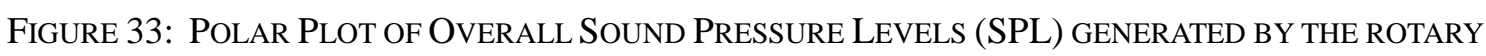
MOTION OF THE WIND TURBINE BLADES.

FigURE 34: SCHEMATIC SHOWING Flow OF INFORMATION IN DESIGN OPTIMIZATION TOOLKIT.............. 33

FIGURE 35: SHAPE DEFORMATION GRID PRODUCED BY SCULPTOR AND ASD GRID SHOWN BY RED, GREEN AND BLUE LINES WITH CONTROL POINTS AS NODES ON THE GRID.

FIGURE 36: SHAPE MODIFICATIONS TO THE TURBINE BLADE GENERATED BY MOVING THE CONTROL POINTS

Figure 37: PARAMETERIZATION OF Wind TURbine Blade GeOMETRY FOR DESIGN OPTIMIZATION STUDY.

FiguRe 38: Design LANDSCAPE SHOWING POWER GENERATED BY EACH DESIGN AND ITS NOISE CHARACTERISTICS. THE DESIGN LANDSCAPE ALSO SHOWS A SUBSET OF DESIGNS (PARETO-FRONT) WHERE DESIGNS HAVING THE NON-DOMINATED SOLUTIONS EXIST.

Figure 39: AIRFOIL CROSS-SECTION SHAPES FOR DiFFERENT DESIGNS EXTRACTED FROM DESIGN Space. Cross-Section of BSDS Shape is Shown in Black. Other Designs are SHOWn IN Red.

FIGURE 40: (-CP) COMPARISONS OF DIFFERENT CROSS-SECTIONS OF THE ORIGINAL BSDS WIND TURBINE

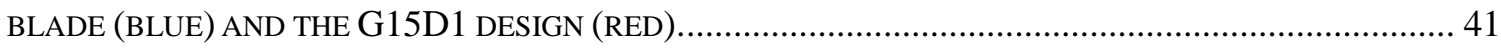

FIGURE 41: (-CP) COMPARISONS OF DIFFERENT CROSS-SECTIONS OF THE ORIGINAL BSDS(BLUE) WIND TURBINE BLADE AND THE G15D3 DESIGN (RED)...

FIGURE 42: (-CP) COMPARISONS OF DIFFERENT CROSS-SECTIONS OF THE ORIGINAL BSDS (BLUE) WIND TURBINE BLADE AND THE G14D1 DESIGN(RED).

Figure 43: Polar Distribution of RMS PRESSURE COMPARISONS FOR THE ORIGINAL BSDS BLADE AND THE THREE DESIGNS.

Final Report

$\mathrm{V}$

CRAFTR-08.2011.030

Prime Contract No. DOE-DE-EE0003269

Combustion Research and Flow Technology, Inc. 
This page left blank intentionally.

Final Report

vi

CRAFTR-08.2011.030

Prime Contract No. DOE-DE-EE0003269

Combustion Research and Flow Technology, Inc.

\section{Page C-7}

Final Report | DOE Award No. DE-EE0003269 


\section{Chapter 1 INTRODUCTION}

The activities in this program were carried out to support NCPA in its program objectives: (i) to carry out Large Eddy Simulation (LES) to characterize wind turbine inflow conditions in support of real-time measurements taken at NCPA utilizing low cost Sonic Detection and Ranging - SODAR and/or an array of passive, ground-based pressure sensors measurements (ii) to develop a blade design optimization procedure for the design of a low noise rotor through shape optimization of blade geometry.

Large-eddy simulations were performed by researchers at CRAFT Tech of the set up as designed by NCPA in order to emulate an atmospheric boundary layer flow in the wind tunnel. A low-speed flow was made to pass over a backward-facing step (of 3" in height) and let to reattach, representing the physics of wind flow over a hill-like terrain. Subsequent to the reattachment, a new, "thick" boundary layer was established. This boundary layer will be influenced by the intermittent, upstream, wake-like effects of the unsteady vortex shedding from the backward-facing step and hence would emulate the presence of gusts in an atmospheric boundary layer. In addition the temperature gradient in the atmospheric boundary layer needs to be modeled. This effect is modeled by heaters placed on the bottom wall of the wind tunnel. Details of the simulation including the set-up and results are discussed in Chapter 2.

The other objective of this program is to design wind turbines where noise associated with the operation of wind turbines is reduced and develop a computational framework that aids in achieving this end. With high wind velocities and particularly at night audible sound levels can be high. However for wind speeds in the turbine design range and during the daytime measured audible noise is very low. Despite this complaints from the surrounding public continue to be received and fluctuations in the sub-audible frequency range can pose health risks to neighboring communities. This problem can only continue to deteriorate as more wind turbines come on line. To achieve growth in sustainable energy using wind power requires designs where the noise issue is alleviated. In Chapter 3, we discuss a computational framework for the design and development of wind turbines with reduced noise levels. Results from a brief design optimization study are also discussed in this Chapter.

Final Report 1

CRAFTR-08.2011.030

Prime Contract No. DOE-DE-EE0003269 Combustion Research and Flow Technology, Inc. 


\section{Chapter 2 LARGE-EDDY SIMULATION OF ATMOSPHERIC BOUNDARY LAYER}

This chapter will present the results from the large-eddy simulation of the flow behind a backward-facing step as simulated in the NCPA wind tunnel in order to emulate the effects of gusts in an atmospheric boundary layer.

\subsection{Overview}

Figure 1 shows a schematic of the set up as designed by NCPA in order to emulate an atmospheric boundary layer flow in the wind tunnel. A low-speed flow will be made to pass over a backward-facing step (of 3" in height) and let to re-attach. Subsequent to the reattachment, a new, "thick" boundary layer will be established. This boundary layer will be influenced by the intermittent, upstream, wake-like effects of the unsteady vortex shedding from the backwardfacing step and hence would emulate the presence of gusts in an atmospheric boundary layer. This would nominally be representative of a "daytime" atmospheric boundary layer.

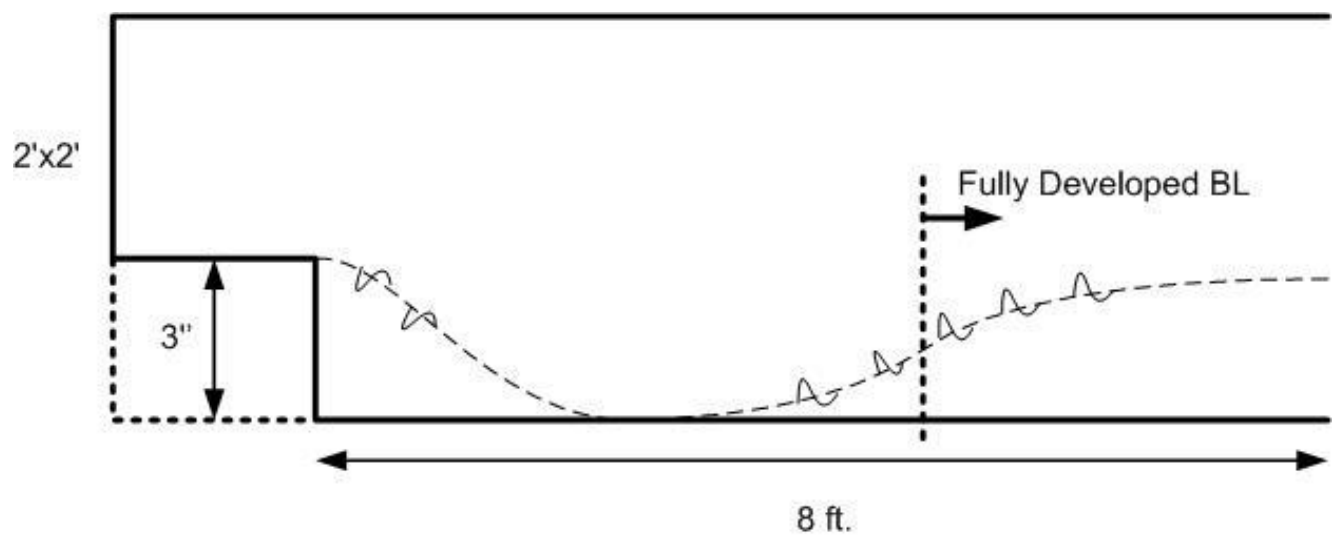

Figure 1: Experimental setup of the backward-facing step configuration in the subsonic wind tunnel.

During nighttime, the earth's surface is heated up and hence would also result in freeconvective eddies due to the temperature gradients present in the boundary layer. This effect was to be modeled by heaters placed on the bottom wall of the wind tunnel. The heaters necessary for the above have been procured that have a maximum heat capacity of $5 \mathrm{~W} / \mathrm{sq}$. in. The maximum speed at which the subsonic wind tunnel can be operated is $20 \mathrm{~m} / \mathrm{s}$.

Final Report 2

CRAFTR-08.2011.030

Prime Contract No. DOE-DE-EE0003269 Combustion Research and Flow Technology, Inc. 
CRAFT Tech was tasked with performing RANS simulations of the above to determine the optimal run parameters for the above two scenarios. A RANS simulation of the setup has been completed. The flow speed chosen is $30 \mathrm{ft} / \mathrm{s}$ that correspond to a Mach number of $\sim 0.025$. Figure 2 shows the mean streamwise velocity contours. The separated flow region downstream of the step can be seen. The reattachment of the shear layer to form a boundary layer can also be seen. Figure 3(a) shows the profile of the mean streamwise velocity, $U$ along the downstream direction close to the bottom wall. The separated flow region extends to a downstream distance of $\mathrm{x} / \mathrm{H} \sim 5$ that correlates quite well with experiments and the accepted approximate reattachment point. The boundary layer profile of $U$ at the end of the $8 \mathrm{ft}$ section downstream of the step is shown in Figure 3(b). The boundary layer thickness at this station is about 2 step heights.

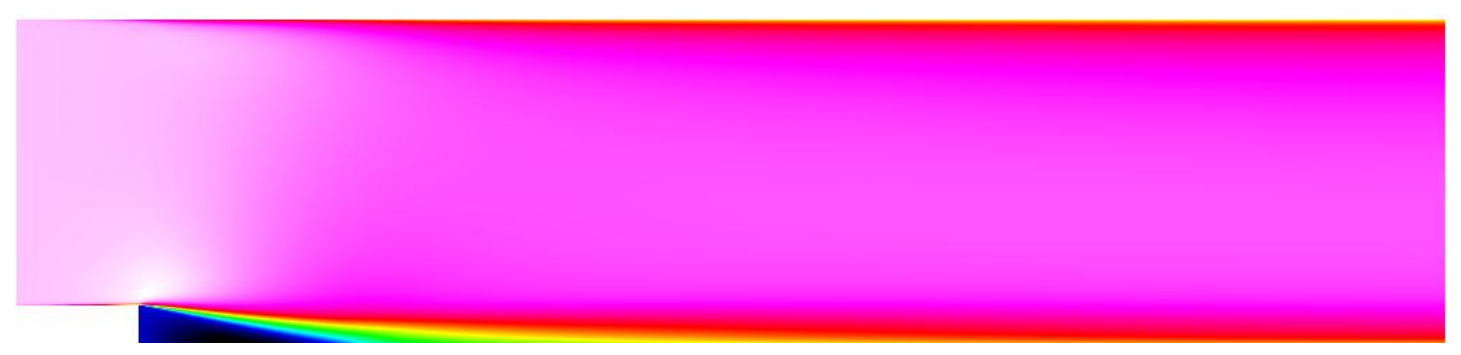

Figure 2: Mean streamwise velocity, $U$ in the mid-span plane of the wind tunnel.

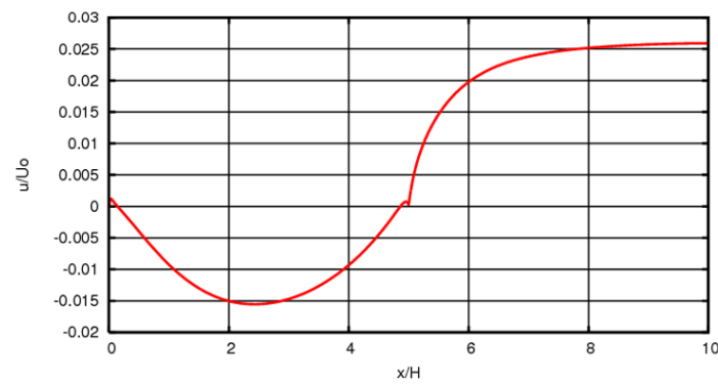

(a) Streamwise velocity close to the bottom wall showing the separated region behind the step.

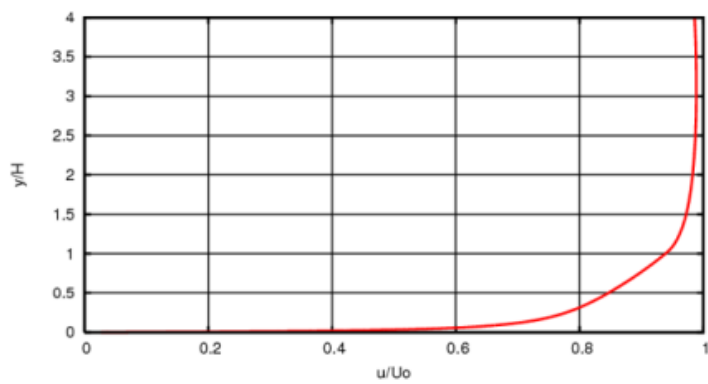

(b) Boundary layer profile at the end of the 8 ft. section downstream of the step.

Figure 3: Streamwise velocity, U profiles (a) showing the separated region, and (b) the boundary layer profile.

Final Report 


\subsection{Large-Eddy Simulations}

A 3D, unsteady LES simulation was also initiated for the setup. The extents of the computational domain in the streamwise, $\mathrm{x}$, and wall-normal direction, $\mathrm{y}$, is identical to those used for the RANS simulation. The spanwise, $z$, extent of the domain is chosen to be one step height, $\mathrm{H}$ and periodic boundary conditions (BCs) will be applied in this direction. The maximum structure size in any direction is expected to be smaller or utmost about the size of the step height, $\mathrm{H}$ and hence the spanwise extent size of one step height is justifiable with periodic BCs. The inflow velocity corresponding to $30 \mathrm{ft} / \mathrm{s}$ was used. The approximate time step for this simulation was less than 1.0e-7 s. Such a small time step would result in about 3 million steps for one flow through time period of the simulation. Based on the low frequency gust phenomena expected in the simulation at least 3-6 such time periods of unsteady flow data would be necessary to obtain time-converged statistics that would make the simulation prohibitively expensive.

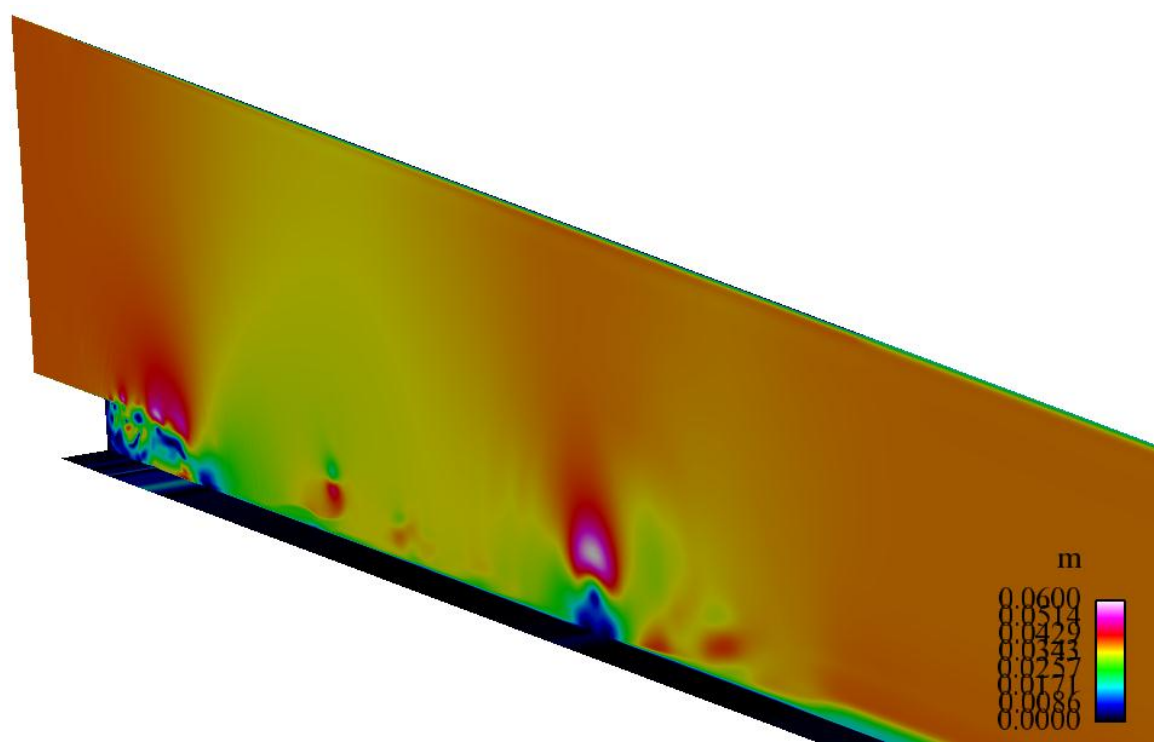

Figure 4: Mean contours of the Mach number in the mid-span plane and the bottom wall for the 3D LES.

Hence, after consultation with NCPA personnel, the flow speed in the wind tunnel was increased to $45 \mathrm{ft} / \mathrm{s}$. Further, a coarser grid in the wall-normal direction (that mainly determines

Final Report 4

CRAFTR-08.2011.030

Prime Contract No. DOE-DE-EE0003269 Combustion Research and Flow Technology, Inc. 
the time step based on the diffusion time scale in the boundary layer) was made that would facilitate increasing the average time step of time advancement in the solution. The resulting time step was increased to about $\sim 1.0 \mathrm{e}-6 \mathrm{~s}$. Figure 4 shows an instantaneous snapshot of the Mach number in the mid-span plane and along the bottom wall in the simulation. The formation of eddies in the shear layer over the step and in the re-circulating region can be clearly seen. Also, the roll-up of a large-scale eddy about the size of a step height can be discerned that has convected downstream to a distance of $\mathrm{x} \sim 4 \mathrm{ft}$. This eddy is representative of a "gust" that forms in an atmospheric boundary layer. The above simulation showed that the predominant large-scale structure of the flow in the spanwise direction is very nearly two-dimensional in nature. Thus, a 2D unsteady LES simulation was also initiated along with the above $3 \mathrm{D}$ simulation. The time step of time-advancement in this simulation was 1.0e-6 s. Figure 5 show a few instantaneous snapshots of the Mach number contours that are representative of the flow evolution. The unsteady eddies in the shear layer; re-circulating flow region downstream of the step and the large-scale "gust" that has convected almost to the end of the domain can be seen. However, this simulation resulted in large 2D, vortical structures rolling over the boundary layer that resulted in "unphysically" large pressure fluctuations on the wall (OASPL $130 \mathrm{dBs}$ ). The lack of threedimensionality or the prevention of breakdown in the z-direction vorticity into the other two directions resulted in these rather "unrealistic" large, 2D vortical structures. These 2D structures were hence simply convected down the boundary layer (as seen from the earlier Figure 5) rather than redistributed/re-aligned into vorticity in the other directions that would have resulted in smaller scale turbulent structures.

Final Report 


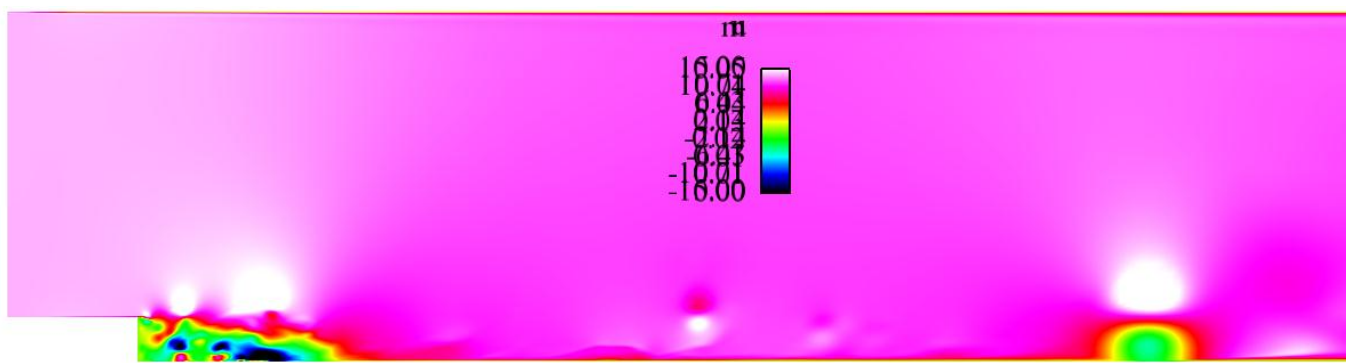

(a)

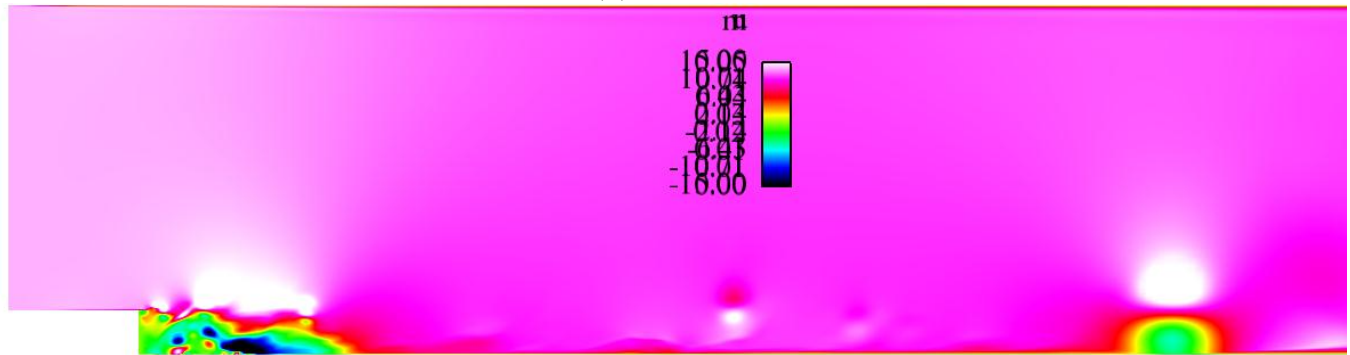

(b)

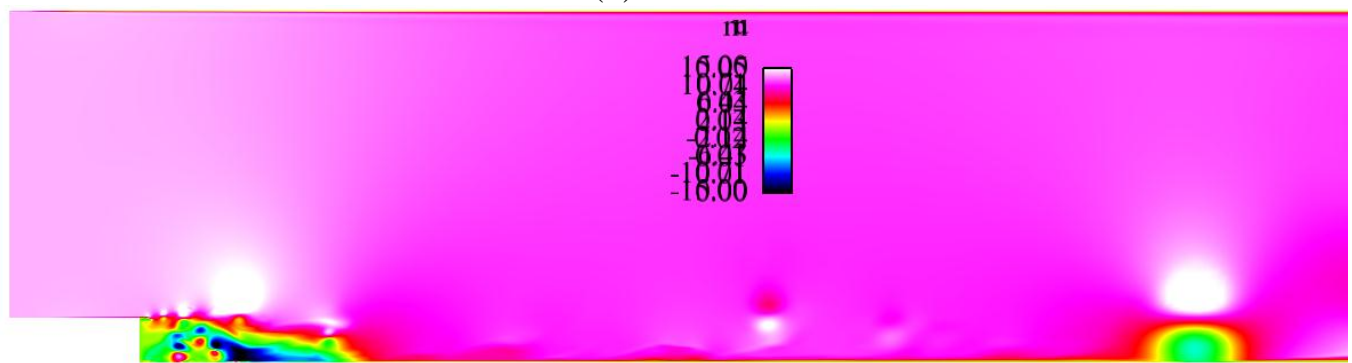

(c)

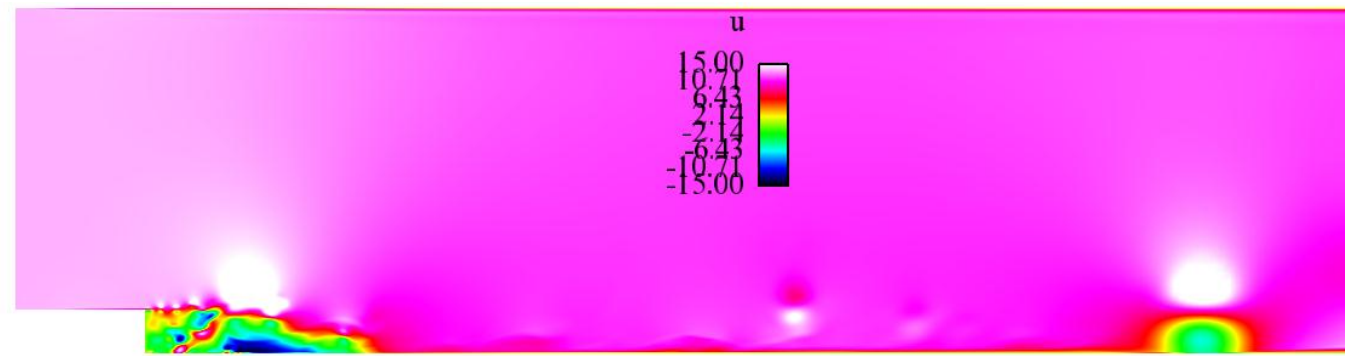

(d)

Figure 5: Mean streamwise velocity, $U$ contours for the 2D LES at four representative instants in time showing the development of the eddies/gusts in the boundary layer downstream of the step.

Hence the coarse grid 3D, LES was again pursued. Trace points were placed (every 3 inches) along the bottom wall to record the pressure fluctuations from the boundary layer flow

Final Report

Prime Contract No. DOE-DE-EE0003269

Combustion Research and Flow Technology, Inc. 
over them. In addition the temperature gradient in the atmospheric boundary layer needs to be modeled. This effect is modeled by heaters placed on the bottom wall of the wind tunnel. The heaters keep the wall at an isothermal state of $325 \mathrm{~K}$. A LES, of this situation was also performed with the above isothermal condition for the wall temperature. The experiment at NCPA was conducted at speeds of 15, 20, 25 and $30 \mathrm{ft} / \mathrm{s}$. The CFD simulations were run at a speed of $45 \mathrm{ft} / \mathrm{s}$ so that the low Mach number effects of the flow when using a compressible solver such as CRAFT CFD can be alleviated. In order to keep the simulation cost low, only 10 points were used in the transverse or spanwise direction spanning a length of 3" (i.e., equal to the step height). Note this resolution is very coarse and only resolves scales greater than 0.3 " in size. The grid uses 630 points in the streamwise direction and 250 points in the wall normal direction with 150 of them concentrated in a step height above the bottom wall. Grid points are concentrated along the bottom wall and near the step as shown in Figure 6. The total grid size is about 1.7 Million. The boundary layer again severely restricted the time step size in the simulation to $1.0 \mathrm{e}-$ 6 s. The simulation was run for a Million steps to a total physical time of $1 \mathrm{sec}$. The computational cost of the simulations required 350 hours of CPU time while using 64 processors on a Linux Beuwolf cluster. Figure 7 shows four instantaneous snapshots of the flow field (Mach number contours) along the mid-span plane of the simulation domain. In contrast to the instantaneous flow contours shown in Figure 5, this 3D simulation shows a more "realistic" turbulent boundary flow field with more three-dimensionality rather than the $2 \mathrm{D}$, large vortical structures seen earlier with the unsteady, 2D simulation. Also the cross-stream contours (shown in Figure 8) of the flow field showed the evolution of three-dimensional structure to the boundary layer flow.

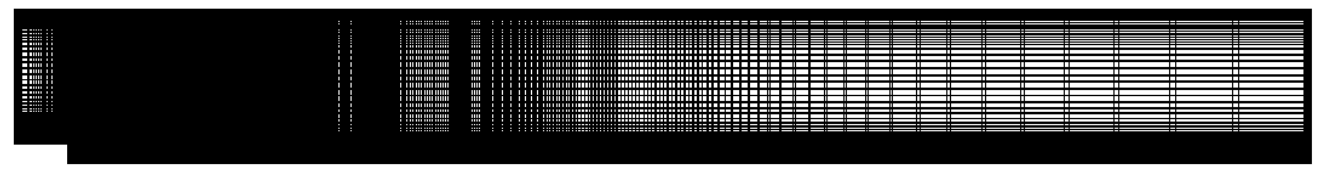

Figure 6: Schematic of the grid as cut through the mid-span of the computational domain used for the CFD simulations.

Final Report

Prime Contract No. DOE-DE-EE0003269

Combustion Research and Flow Technology, Inc. 

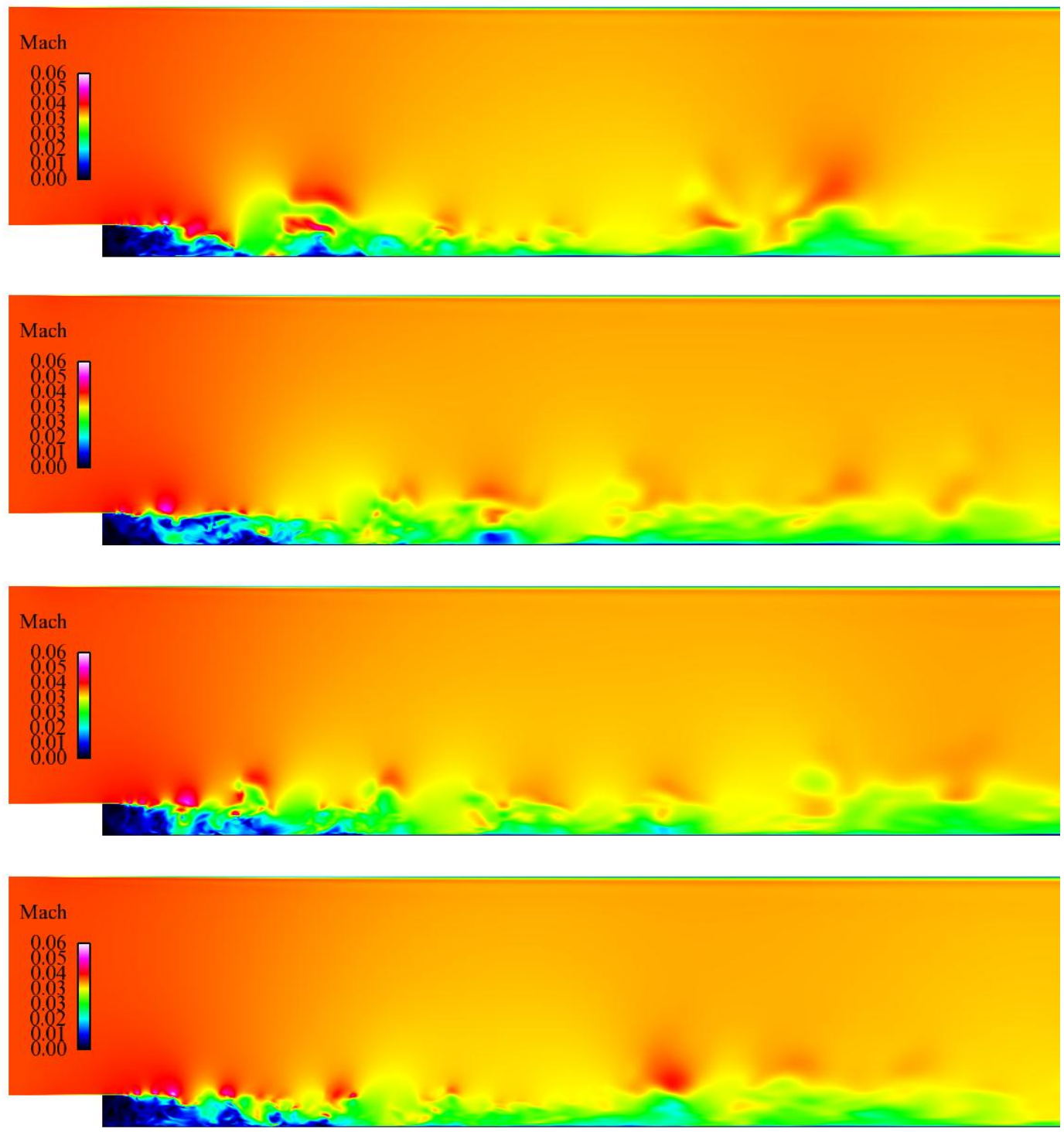

Figure 7: Instantaneous snapshots of the Mach Number Contours on the Mid-span Plane of the Simulation at four instants in time showing the Unsteady Development of the Boundary Layer on the Bottom Wall.

Final Report 8

CRAFTR-08.2011.030

Prime Contract No. DOE-DE-EE0003269

Combustion Research and Flow Technology, Inc. 


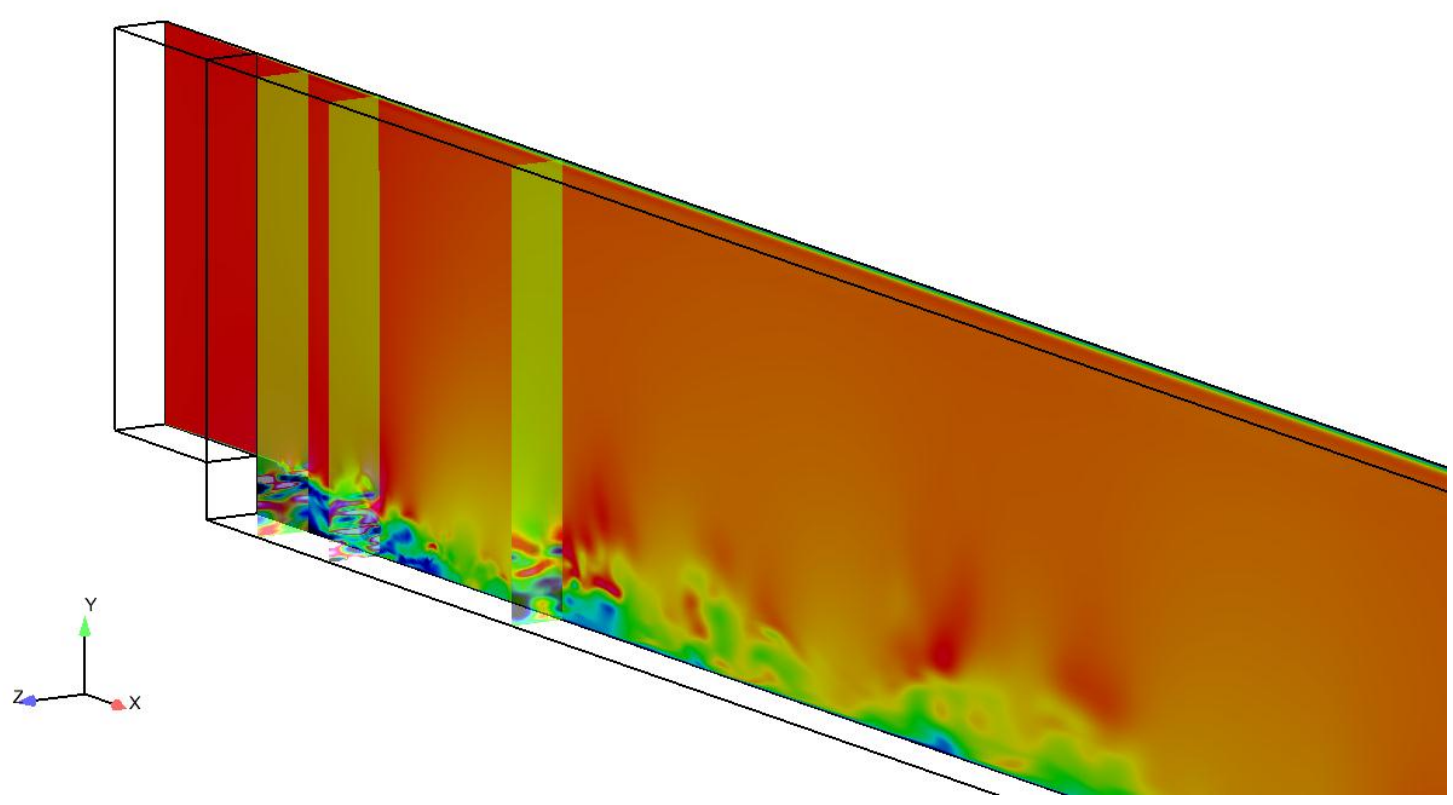

Figure 8: Instantaneous snapshots of the Mach Number Contours on the Mid-span Plane of the Simulation along with cross-stream contours of $W$-velocity at $x=5,12,20$ " from the step showing the Unsteady Development of the Boundary Layer on the Bottom Wall.

Figure 9 shows the mean streamwise velocity very close to the wall (1st point of the wall) that is representative of the extent of the re-circulation region downstream of the step. For both the adiabatic and the isothermal wall conditions, the re-circulation zone extends to a streamwise extent of $\mathrm{x} / \mathrm{H} \sim 5$. This distance agrees well with the extent observed in other simulations and experimental data. The isothermal condition shows a slightly smaller re-circulation zone than the adiabatic wall condition. Figure 10 and Figure 11 show the wall normal distribution of the mean streamwise velocity for the adiabatic and isothermal wall conditions respectively. For $\mathrm{x} / \mathrm{H}<5$, the profiles show negative streamwise velocity regions representative of the re-circulating flow downstream of the step. For $\mathrm{x} / \mathrm{H}>18$, the profiles are very nearly similar representative of a reestablished boundary layer flow far downstream of the backward-facing step. These profiles also show that downstream of the step the mean streamwise velocity away from the wall (in the freestream) decreases to about 7/8th of the freestream value ahead of the step as a consequence of mass conservation. Figure 12 shows the wall normal distribution of the mean temperature for the isothermal wall flow field. Finally, Figure 13 compares the boundary layer profiles at the

Final Report 
end of the $8 \mathrm{ft}$ section for the two wall conditions. This boundary layer profile for the adiabatic wall condition is slightly fuller than that for the isothermal wall condition.

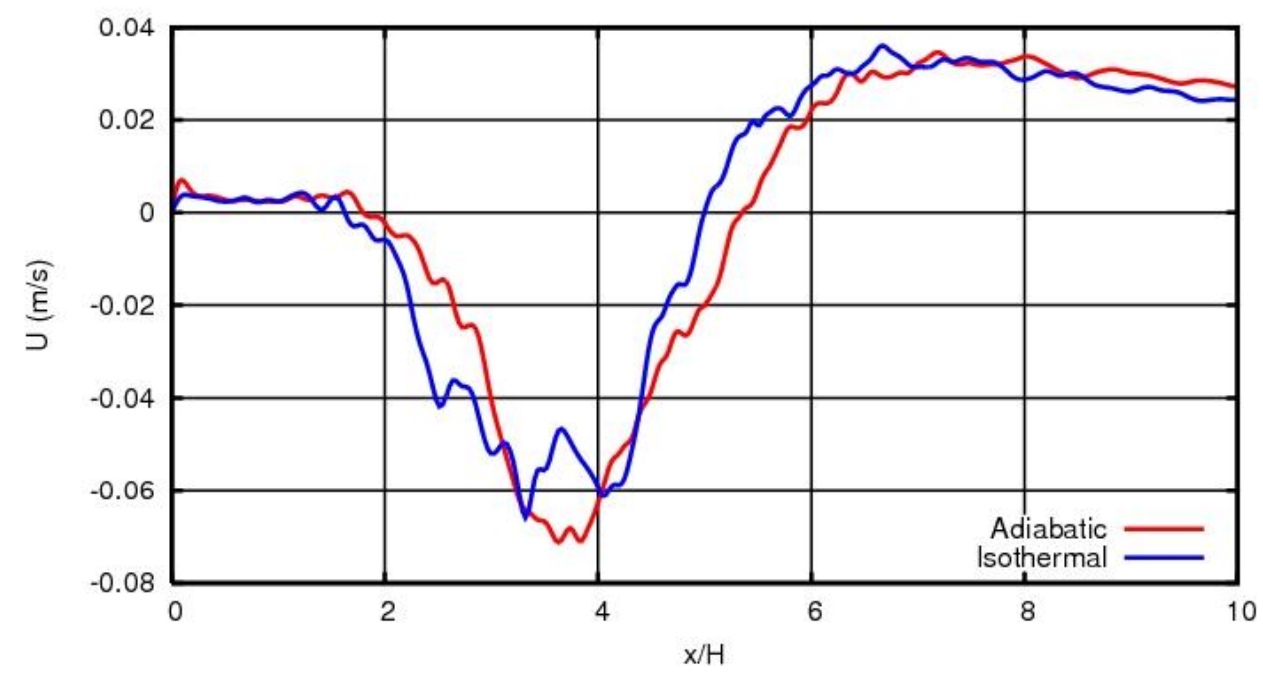

Figure 9: Mean Streamwise Velocity Distribution Close to the Wall Representative of the Separated Flow Region in the Flow Field.

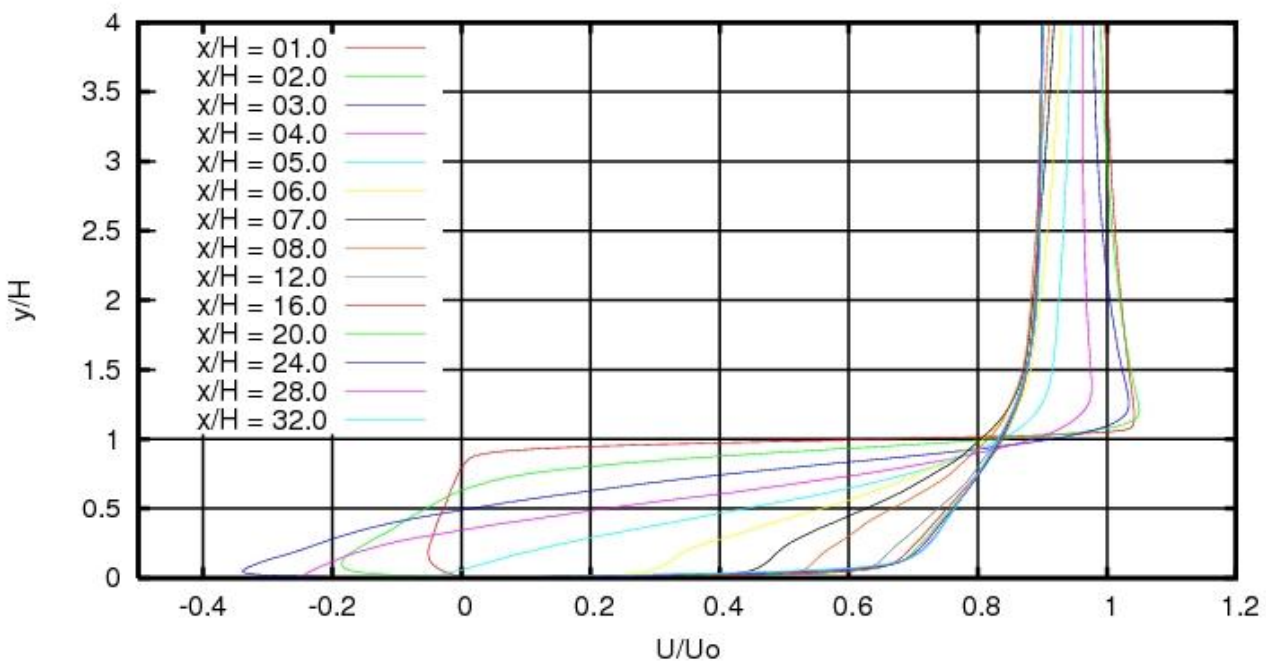

Figure 10: Mean Streamwise Velocity Distribution along the Wall Normal Direction(y) at a few streamwise Stations (for the Adiabatic Wall Boundary Layer).

Final Report 


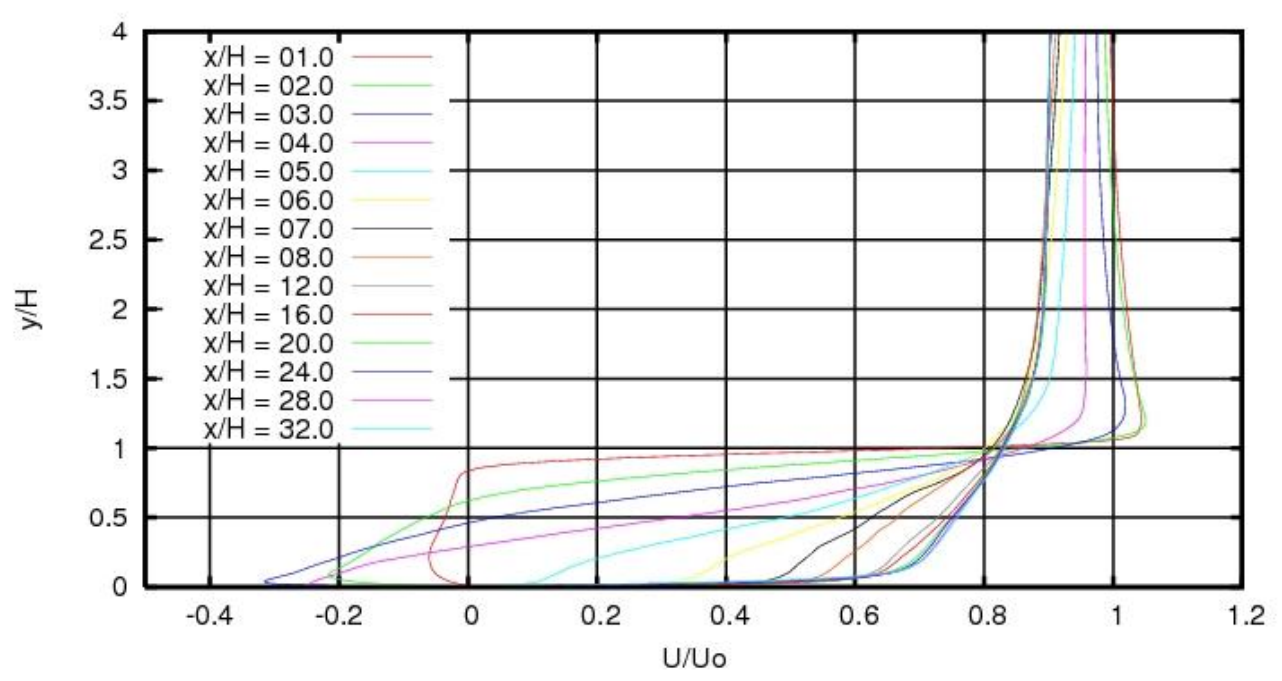

Figure 11: Mean Streamwise Velocity Distribution along the Wall Normal Direction at a few Streamwise Stations (for the Adiabatic Wall Boundary Layer).

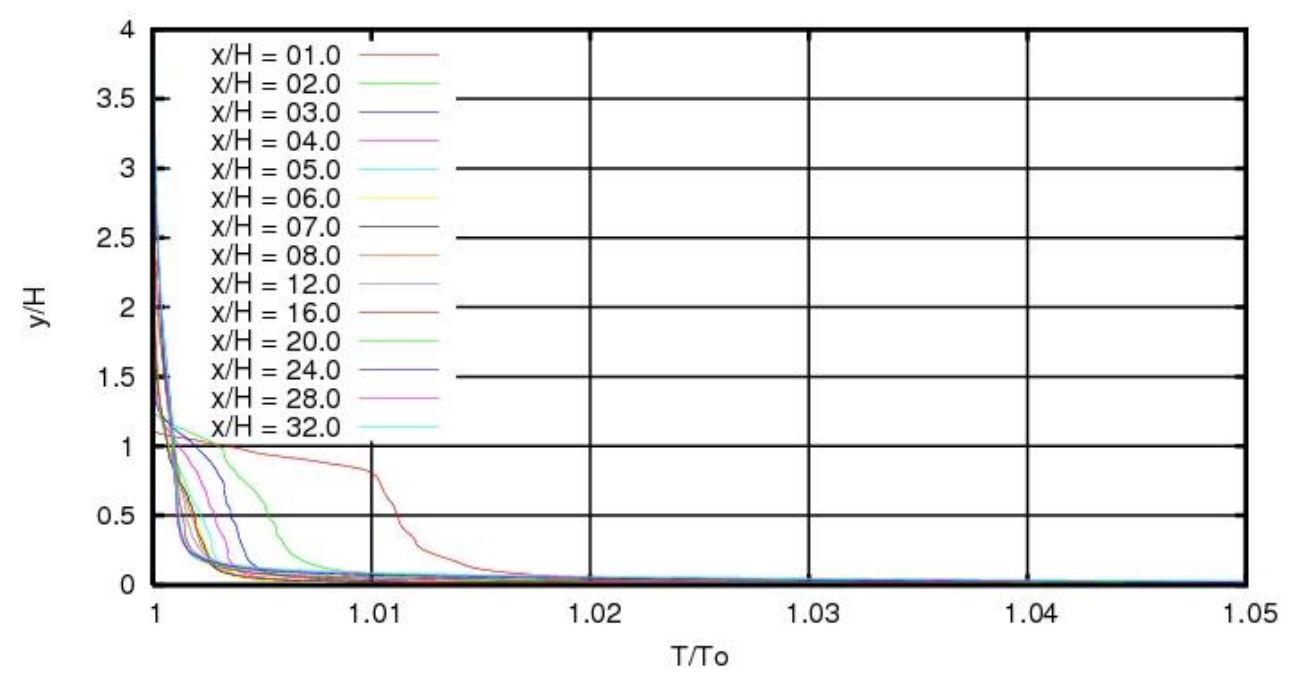

Figure 12: Mean Temperature Distribution along the Wall Normal Direction at a few Streamwise Stations (for the Isothermal Wall Boundary Layer).

Final Report

Prime Contract No. DOE-DE-EE0003269

Combustion Research and Flow Technology, Inc. 


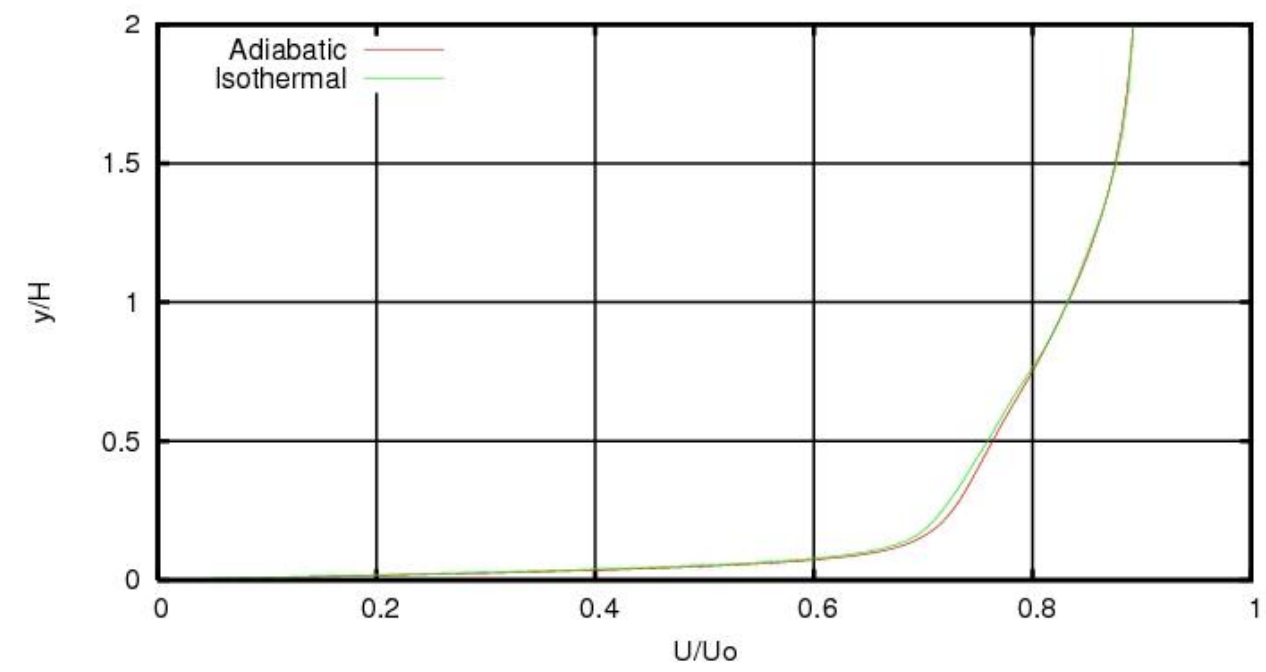

Figure 13: Boundary Layer Profile at the end of the $8 \mathrm{ft}$ section of the wind tunnel.

Post-processing of the pressure spectra (in Figure 15) show a dominant tone of about 5 $\mathrm{Hz}$ that implies about 5 cycles of this tone are available in the total time sample (of $1 \mathrm{sec}$ ). The OASPL distribution (in Figure 14) shows that the effect of a heated boundary layer increases the SPL on the wall by 2-3 dBs. Figure 15 shows the pressure spectra on the wall for the adiabatic wall case. The experimental data for 15 and $30 \mathrm{ft} / \mathrm{s}$ show an increase in the noise levels of $\Delta \mathrm{dBs}$ $\sim$ 10. If this mean speed scaling is valid further to higher speeds, then the SPL spectra from the CFD simulation at a speed of $45 \mathrm{ft} / \mathrm{s}$ is reasonable considering a further increase of $\Delta \mathrm{dBs} \sim 10$. The simulation spectra at $45 \mathrm{ft} / \mathrm{s}$ also show similar low frequency content of $\sim 5 \mathrm{~Hz}$ dominant tone and a harmonic of $\sim 10 \mathrm{~Hz}$. The middle range of frequencies between $10-100 \mathrm{~Hz}$ is overpredicted by the LES, while the higher range $>100 \mathrm{~Hz}$ is under-predicted. This is probably attributed to the lack of or no resolution for very small boundary layer scales $(<0.3$ " corresponding to frequencies $>100 \mathrm{~Hz}$ ) that prevents the cascade from scales larger (in the middle frequency range). Figure 16 compares the pressure spectra on the wall for the adiabatic and isothermal wall cases. Similar to the adiabatic case, the spectra show dominant low frequency content of $\sim 10 \mathrm{~Hz}$ (slightly higher) for the isothermal wall case. The spectral content at higher frequencies is very similar to the adiabatic wall case.

Final Report 


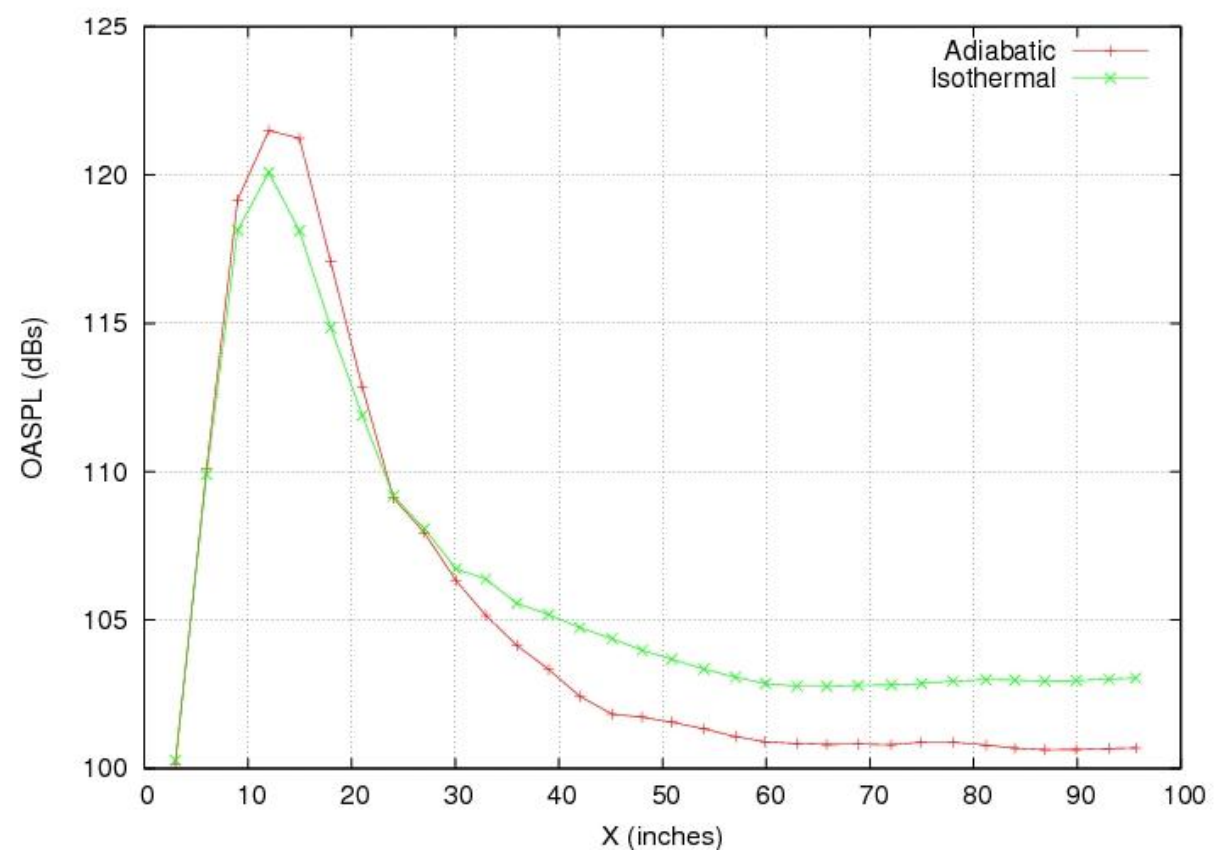

Figure 14: OASPL distribution on the bottom wall.

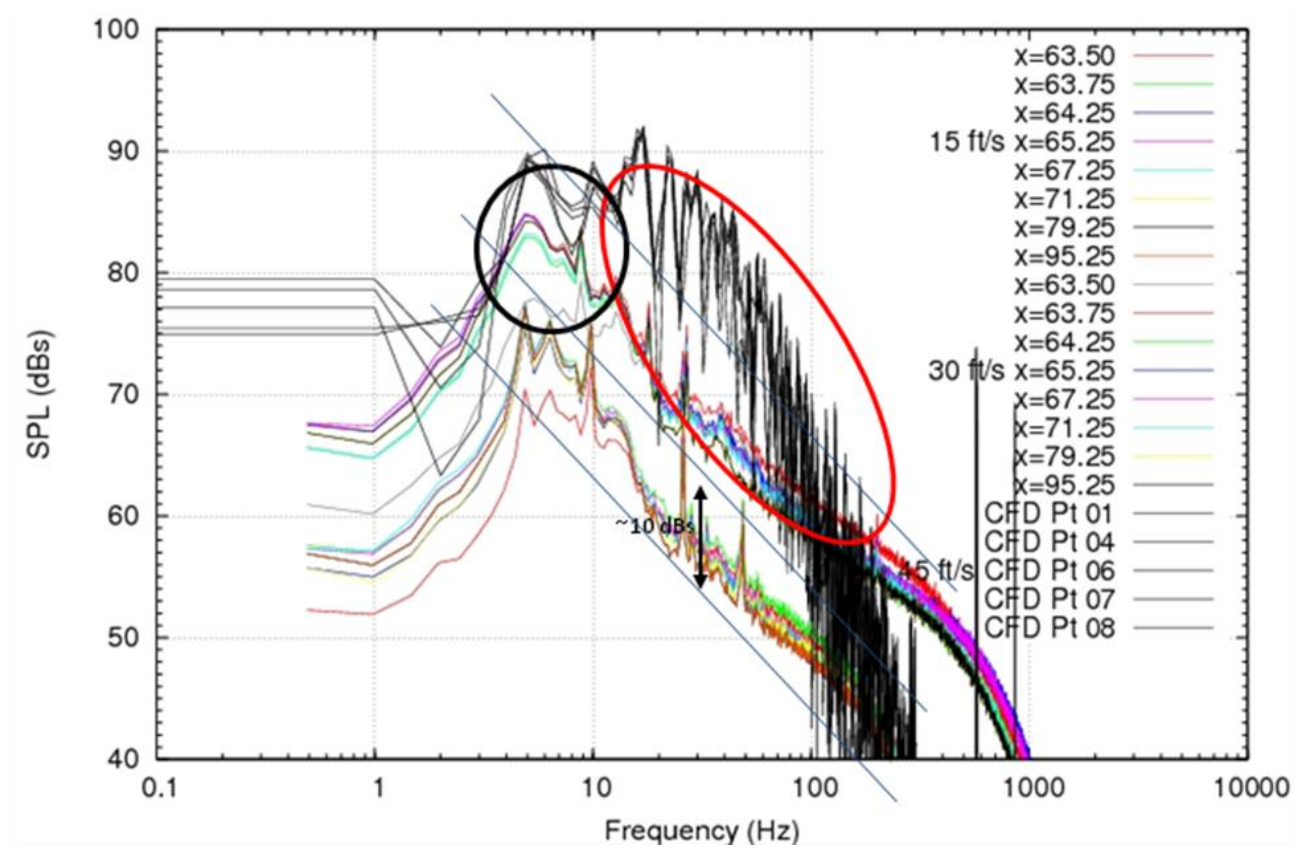

Figure 15: SPL spectra on the bottom wall for the Adiabatic Wall Compared with Available Experimental Data at Lower Speeds.

Final Report

Prime Contract No. DOE-DE-EE0003269

Combustion Research and Flow Technology, Inc. 


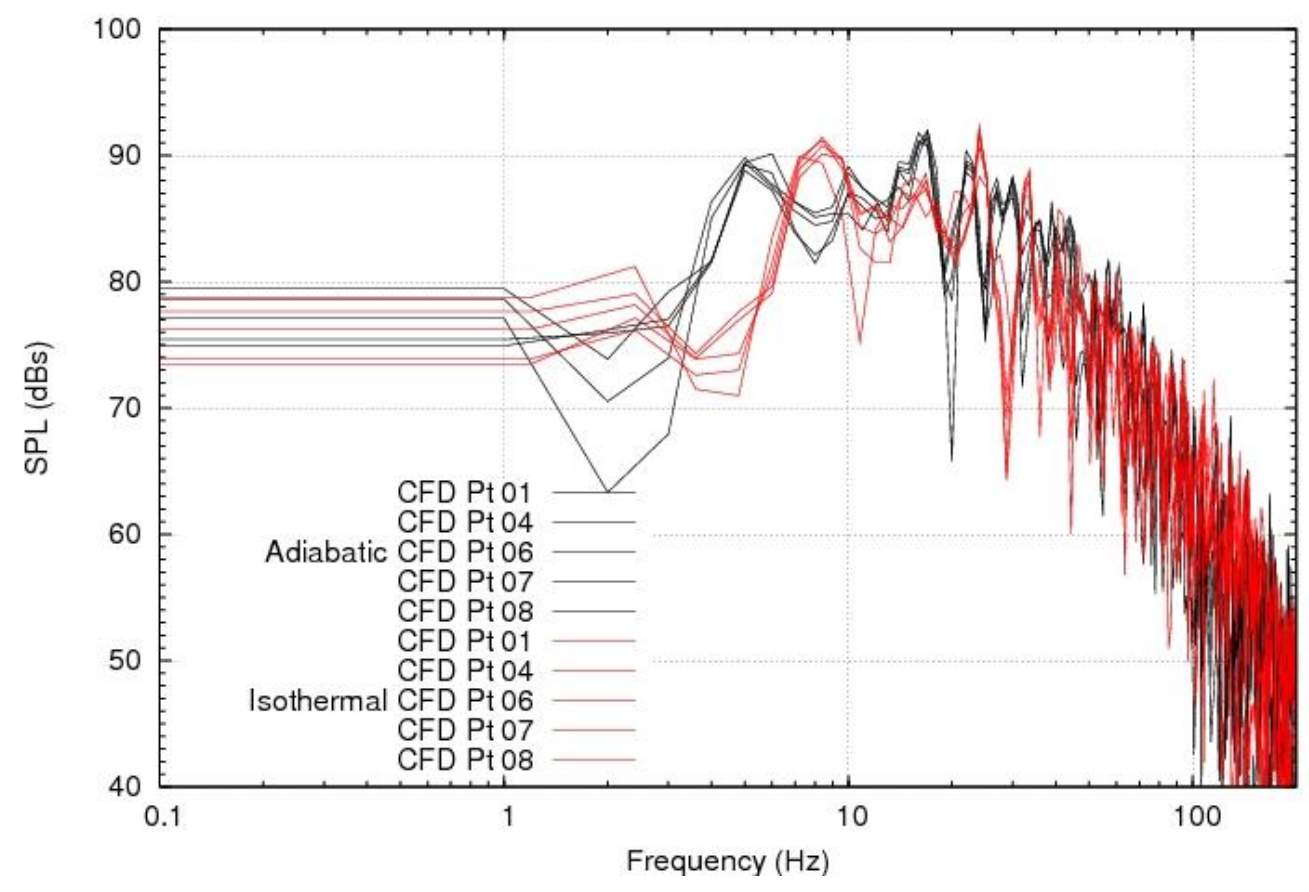

Figure 16: SPL spectra on the bottom wall comparing the Adiabatic and Isothermal Wall Conditions.

\subsection{Summary}

CRAFT $\mathrm{CFD}^{\circledR}$ has been shown to be able to simulate unsteady, 3D boundary layer development with gusts as found in an atmospheric boundary layer. However, the high cost associated with such simulations curtailed the use of a fine-grid and consequently the results are directly influenced by such a cost-cutting, engineering approximation to obtain a solution. The spectral content in the low frequency range is well predicted but the intermediate and high frequency ranges are over-predicted and under-predicted respectively.

Final Report

Prime Contract No. DOE-DE-EE0003269

Combustion Research and Flow Technology, Inc. 


\section{Chapter 3 DESIGN OPTIMIZATION OF WIND TURBINE BLADES}

\subsection{Overview}

One of the primary goals of this program is to reduce noise associated with the operation of wind turbines and develop diagnostic and computational tools that aid in achieving this end. The potential sources of noise that have been identified by researchers [1] studying the aeroacoustics of wind turbines include (i) tonal noise due to the rotation of the turbine blades, which includes thickness and loading noise associated with the rotor blade; (ii) noise due to the generation and propagation of the tip vortex and its subsequent interaction with other structures such as the hub or the tower; (iii) noise due to turbulence and the interaction of the blade with freestream turbulence; and (iv) noise due to vortex shedding from the trailing edge of the rotor blade, which in the case of wind turbines can include flatback airfoil profiles that have a higher susceptibility to the vortex shedding instability and subsequently noise generation.

As part of the computational effort in this program, we will demonstrate the utility of a design optimization based methodology to reduce the noise in wind turbines without adversely affecting performance of the wind turbine. Since a CFD enabled design optimization methodology is computationally intensive, the demonstration will focus on the primary reduction of the tonal noise associated with the rotor which require inputs from steady CFD computations. The baseline design that will be used for this study is the BSDS blade that was developed by the Sandia National Laboratories Blade Systems Design study [2]. It utilizes a flatback airfoil for the inboard sections of the blade and is found to provide improved performance over traditional blades with sharp trailing edges. Furthermore the flatback airfoil based blades have better structural integrity with reduced sensitivity to surface roughness effects such as the presence of surface impurities/debris that can induce boundary layer transition. In this study, we carried out a formal design optimization study for performance enhancement of the turbine blade both in terms of noise reduction and enhancement of aerodynamic power. A summary of the baseline design is provided in the next section. Following that we discuss simulation of the aerodynamic and aeroacoustic performance for the BSDS design. In Section 3.4, we provide details of the design optimization study including the design optimization framework, optimization algorithm

Final Report

Prime Contract No. DOE-DE-EE0003269

Combustion Research and Flow Technology, Inc. 
and parameterization scheme that was used. Lastly, in Section 3.4.4, we discuss results of the design optimization study.

\subsection{Summary of Baseline BSDS Design Utilized for Current Study}

The BSDS blade design [2] has been identified as the baseline design for this study and is summarized in this section. We are working with the $9 \mathrm{~m}$ version that is estimated to achieve an output of $100 \mathrm{~kW}$ on a test turbine. This is a scaled down version of the $1 \mathrm{MW} 50 \mathrm{~m}$ blade design. One of the primary reasons we have started our design optimization studies with this design is that it incorporates several advanced features of turbine blade design technology including structural integrity, flatback airfoil sections, and manufacturing advantages. Details of the blade geometry are discussed in [2] and the blade specifications, airfoil sections as well as the geometry are reproduced here (Figure 17-Figure 19) for the sake of completeness. The blade also has significant twist (12 degrees from root to tip) and a predominant three-dimensional shape that starts off with a circular section at the root, transitions into flatback airfoils in the quarter-span to mid-span region and near-sharp trailing edge at the tip.

\section{BSDS Phase II - Blade Geometry Details - Rev B}

$\begin{array}{rc}\text { Span Length (with Hub): } & 9,000 \mathrm{~mm} \\ \text { Blade Root Location: } & 7.50 \% \text { span } \\ \text { Blade Root Location: } & 675 \mathrm{~mm} \\ \text { Blade Length: } & 8,325 \mathrm{~mm} \\ \text { Root Diameter (7.5\% and 10\% span): } & 20.425 \mathrm{inches} \\ \text { Root Diameter (7.5\% and 10\% span): } & 518.795 \mathrm{~mm}\end{array}$

Figure 17: Specifications of the BSDS Turbine Blade Geometry [2].

Final Report 


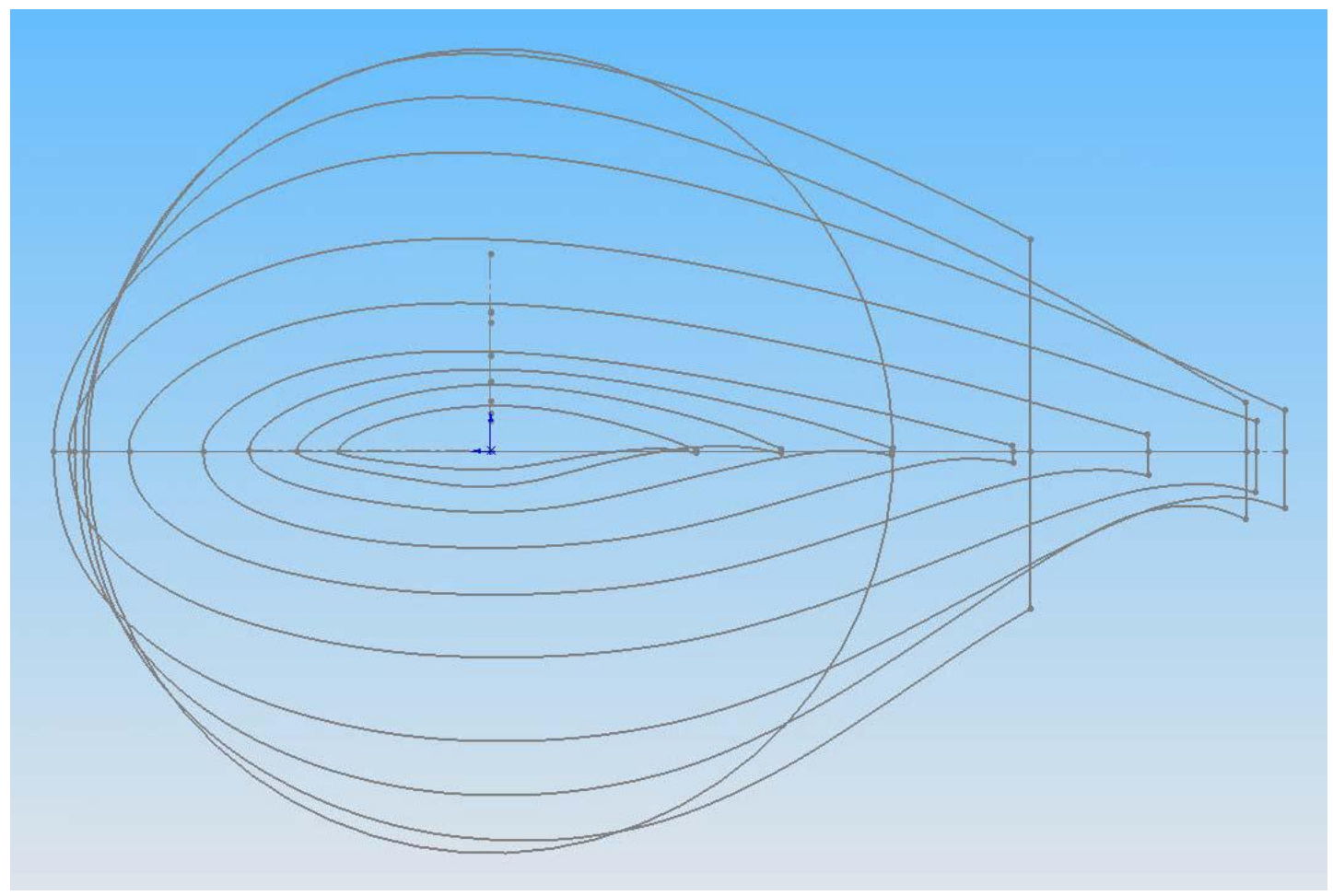

Figure 18: Airfoil Shapes of the different Sections of the BSDS turbine Blade [2].

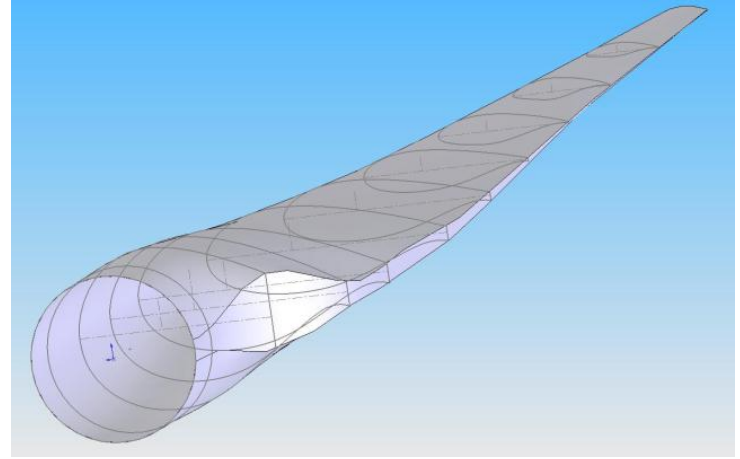

(a)

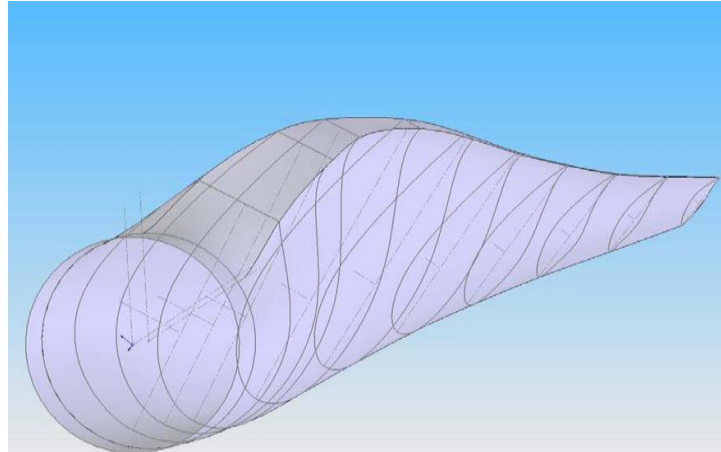

(b)

Figure 19: BSDS Shape Design showing loft, twist and sections [2].

Final Report

Prime Contract No. DOE-DE-EE0003269

CRAFTR-08.2011.030

Combustion Research and Flow Technology, Inc. 


\subsection{Aerodynamic and Aeroacoustic Simulation of Baseline BSDS Blade \\ 3.3.1 Grid Generation}

In our $\mathrm{CRUNCH} \mathrm{CFD}^{\circledR}$ code, we use a multi-element approach wherein an optimal combination of tetrahedral, prismatic, pyramidal and hexahedral cells are used to efficiently solve flows in complex systems by tailoring the mesh to suit the geometrical and physical characteristics of the system. The motivation stems from the use of tetrahedral elements in resolving geometrical complexity, extrusion of prismatic layers from triangulated boundaries in resolving boundary layer phenomena and high aspect ratio hexahedral elements providing adequate clustering in local regions of high directional gradients such as the wake region of a blade. For the case of the BSDS blade, the geometry exhibits considerable complexity - for example the blade profile has a circular shape at the root that transforms into a more conventional airfoil shape section at the tip; the chord also initially elongates as we move from root to tip and then contracts; furthermore the blade has a 12 degree twist from root to tip.

A traditional structured grid approach to a BSDS type wind turbine blade is very cumbersome and leads to skewed cells given the large degree of twist in the blade and the presence of the flatback airfoil sections. Furthermore grid clustering around the blade has to be extended into the far-field leading to a sizeable grid that is computationally expensive. In our multi-element approach the region encompassing the blade is meshed with hexahedral cells primarily to capture the large gradients in pressure and velocity near the surface of the blade. Further away from the blade the computational domain is filled with large tetrahedral cells that help maintain a manageable sized mesh (See Figure 20). The blade itself is enveloped in a C-H hexahedral topology along the span of the blade. A hexahedral block is also used around the hub with significant clustering to permit accurate representation of the boundary layer physics near the hub. The interfacing of the hexahedral block around the hub with the blocks enveloping the blade is shown in Figure 21. A summary of the mesh statistics is provided in Figure 22, indicating an overall mesh size with over 3.2 million elements comprised primarily of hexahedral and tetrahedral cells.

Final Report 


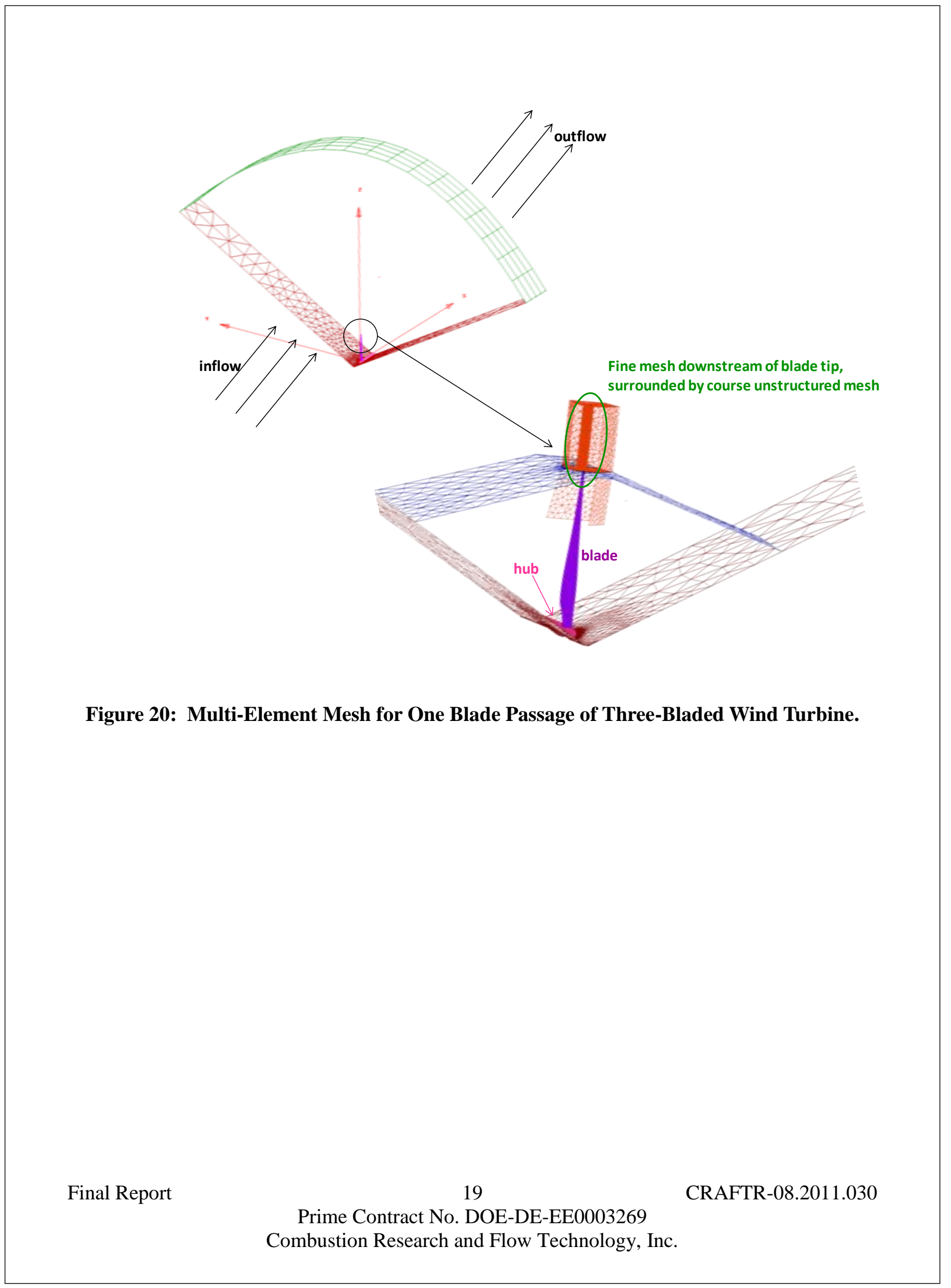




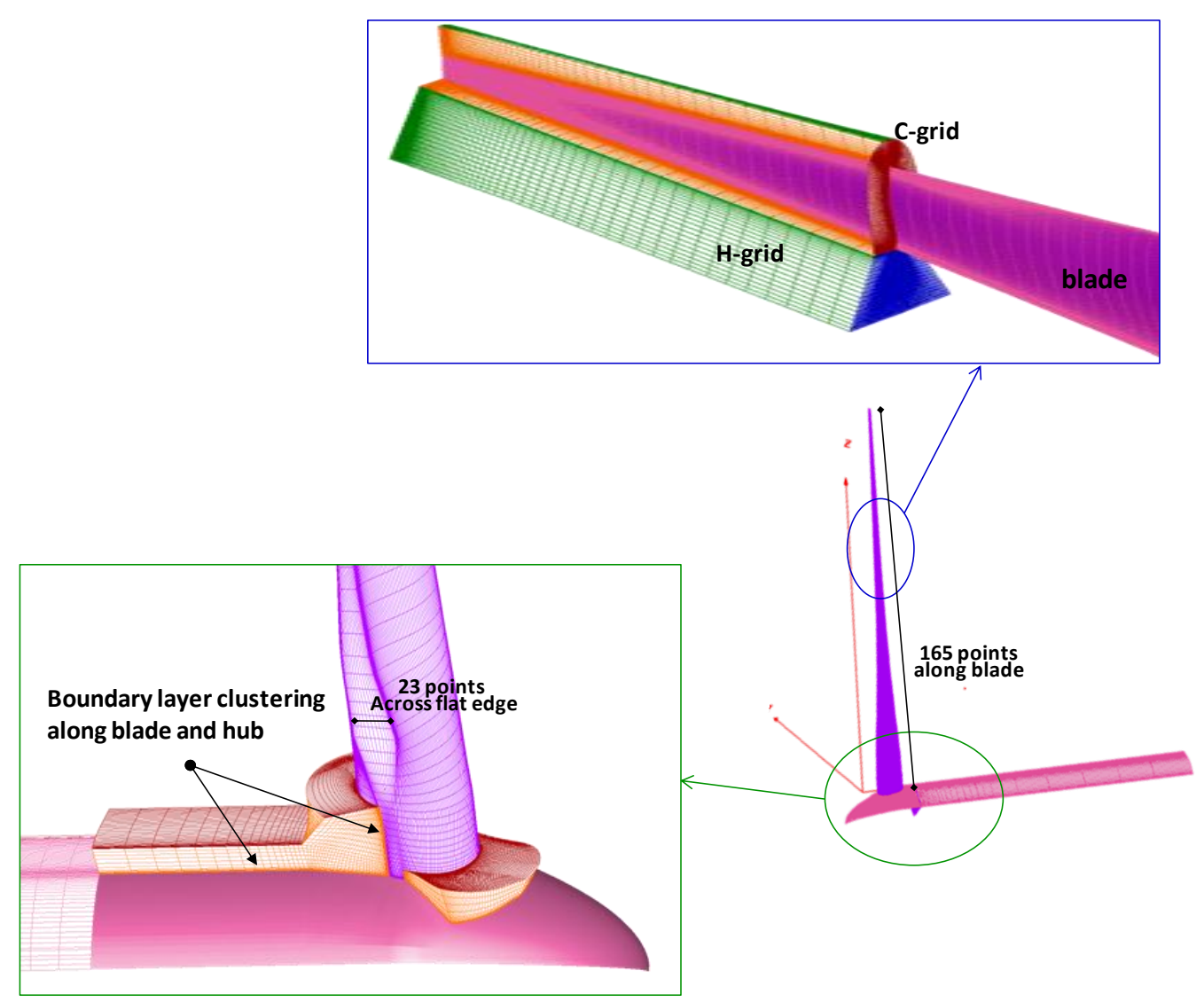

Figure 21: Grid Topology near the hub and the Blade.

\begin{tabular}{|c|c|c|}
\hline M E S H S U & \multicolumn{2}{|c|}{ S U M M A R Y } \\
\hline number of vertices & : & 3281862 \\
\hline number of 4 cells & : & 1479744 \\
\hline number of 5 cells & : & 105225 \\
\hline number of 6 cells & : & 6585 \\
\hline number of 8 cells & : & 2962506 \\
\hline number of 3 bfaces & : & 5632 \\
\hline number of 4 bfaces & : & 59662 \\
\hline
\end{tabular}

Figure 22: Mesh Statistics.

\subsubsection{CFD Simulation Framework}

CFD flow simulations are carried out with the use of a hybrid unstructured solver $\left(\mathrm{CRUNCH} \mathrm{CFD}^{\circledR}\right)$ that allows for the integration of the Navier-Stokes equations on a

Final Report

Prime Contract No. DOE-DE-EE0003269

Combustion Research and Flow Technology, Inc. 
combination of tetrahedral, prismatic, pyramidal and hexahedral cells. For efficient computation of large 3D problems, a parallel framework for distributed memory systems has been implemented, along with a time-marching implicit solution procedure. The sparse implicit matrix is derived by doing an Euler explicit linearization of the first-order flux, and a variety of iterative sparse matrix solvers, e.g. GMRES, Gauss-Seidel procedure, are available in the code (see Ref. [3][4] for details). The parallel framework is implemented by partitioning the grid into sub-domains, with each sub-domain residing on an independent processor. The message passing between processors has been implemented using MPI to provide portability across platforms. Convergence acceleration of the numerical scheme is facilitated by preconditioning the system and unsteady computations are facilitated through a dual time stepping procedure. There are a variety of turbulence models that have been implemented including a two-equation $\mathrm{k}-\varepsilon$ turbulence model, LES sub-grid scale models including algebraic (Smagorinski) and oneequation model. There is also a hybrid RANS-LES model based on the RANS k- $\varepsilon$ model. A key feature of this model is that it provides a smooth transition from regions of high eddy viscosity in the RANS region, to low eddy viscosity in the LES region. Additionally there is also a capability for carrying out moving grid simulations with either a stress-based solver or an algebraic grid motion solver. Furthermore for noise prediction we have a Ffowcs-Williams and Hawkings based formulation that is used to compute the far-field noise from unsteady near-field CFD flow data. The formulation has also been adapted to predict noise from steady turbomachinery simulations. The implementation is discussed in the next Section.

\subsubsection{CFD Results}

Steady state CFD computations were performed on the BSDS blade geometry. The wind turbine consists of 3 blades. For the simulation, symmetry was exploited and the computational domain modeled a single blade embedded in a 120 degree wedge with periodic boundaries. The wind speed in this case was $8.8 \mathrm{~m} / \mathrm{s}$ and the ambient pressure was assumed to be $101325 \mathrm{~Pa}$. Based on the design data, the wind turbine blades have a 1 degree pitch at the tip and rotate at $56 \mathrm{rpm}$. The pressure distribution on the suction side and the pressure side of the wind turbine is shown in Figure 23 and Figure 24, respectively. The pressure distribution on the blades shows a strong spanwise variation. The coefficient of pressure was also computed locally for different spanwise sections utilizing the formula

Final Report 


$$
C_{p}=\left(p-p_{\infty}\right) /\left(\frac{1}{2} \rho_{\infty}\left\{u_{\infty}^{2}+(\Omega r)^{2}\right\}\right)
$$

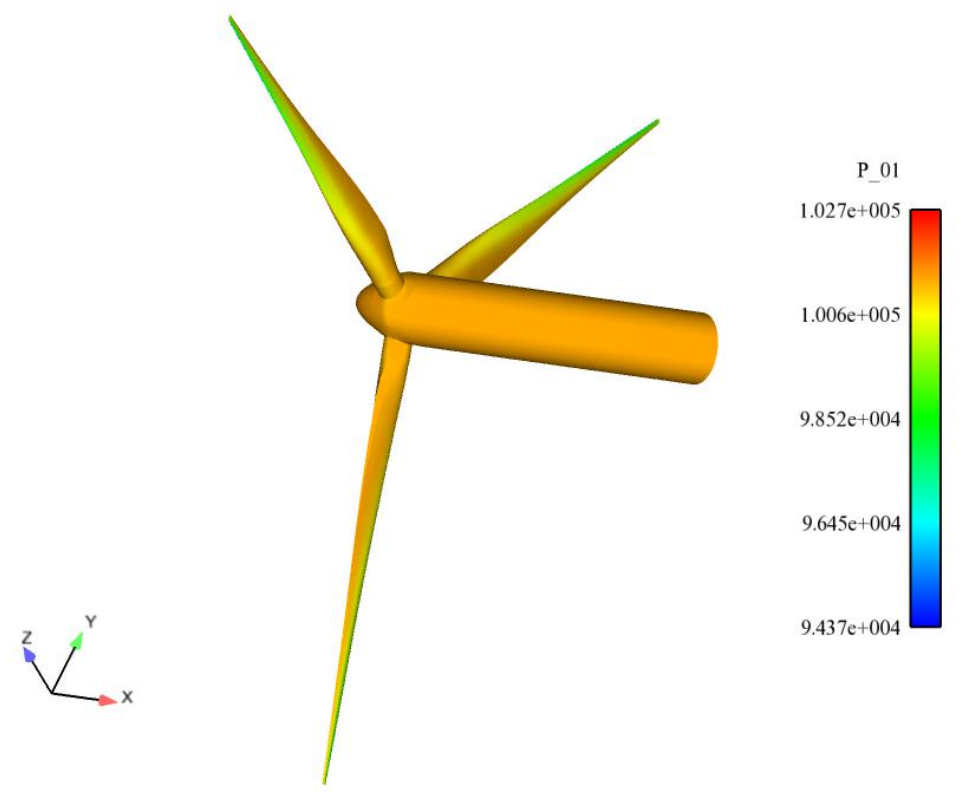

Figure 23: Pressure Distribution on the Suction Side of BSDS Blade.

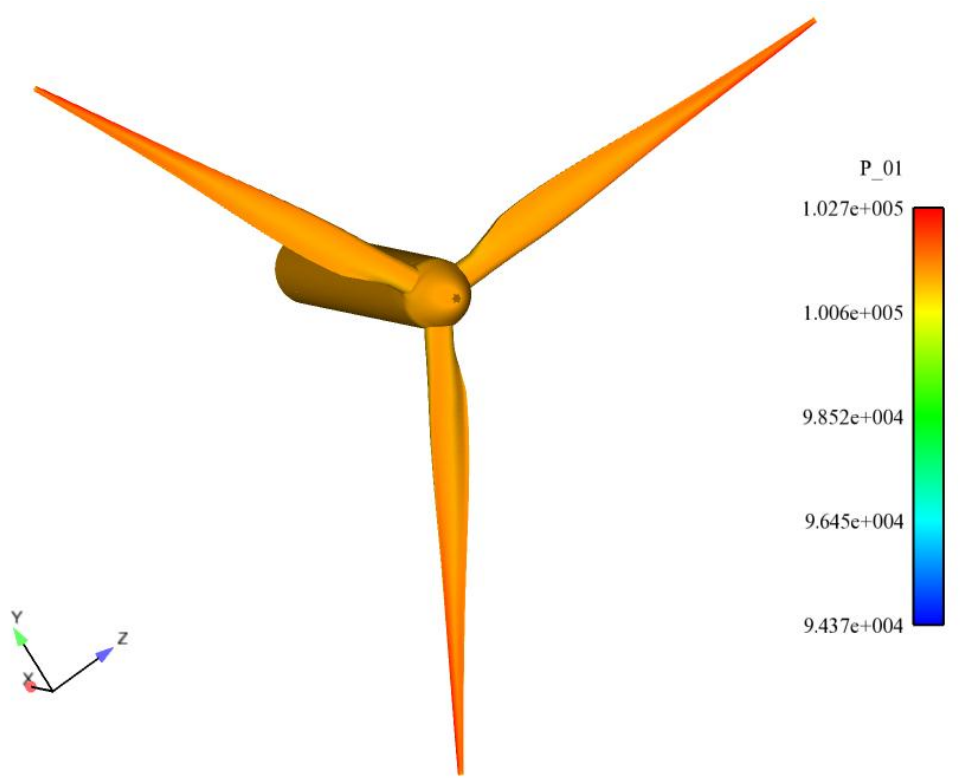

Figure 24: Pressure Distribution on the Pressure Side of BSDS Blade.

Final Report

22

CRAFTR-08.2011.030

Prime Contract No. DOE-DE-EE0003269

Combustion Research and Flow Technology, Inc. 
The coefficient of pressure as a function of chord length is also plotted for five spanwise sections $(20 \%, 45 \%, 65 \%, 85 \%$ and 95\%) in Figure 25. The sections close to the tip show large gradients in pressure on the suction side near the leading edge. It must be pointed out that a highly refined grid was used near the leading edge to accurately capture these large gradients. Furthermore, the predicted aerodynamic power associated with the operation of the wind turbine is very sensitive to the suction side pressure distribution of the blade at spanwise sections near the tip. The predicted aerodynamic power is compared to that compiled by design studies in the BSDS report [2] (also reproduced in Table 1 below) and is in good agreement (See Figure 26) at the wind speed of $8.8 \mathrm{~m} / \mathrm{s}$ and rotor speed of $56 \mathrm{rpm}$ for which the CFD computations were carried out. It should be noted that the turbine performance in [2] was estimated with a preliminary operational model and did not involve rigorous experimentation or high-fidelity analysis.

Table 1: Turbine Performance as a function of Wind speed (Reproduced from [2]).

\begin{tabular}{|cccccc|}
\hline \multicolumn{6}{|c}{ Turbine } \\
Wind & Rerformance as Function of WindSpeed & Aerodyn. & Mechan. & Electric & Axial \\
Speed & Speed & Power & Power & Power & Thrust \\
(m/s) & (rpm) & $(\mathrm{kW})$ & $(\mathrm{kW})$ & $(\mathrm{kW})$ & $(\mathrm{kN})$ \\
\hline 2.9 & 55.5 & 0.5 & 0.0 & 0.0 & 1.6 \\
3.5 & 55.5 & 1.5 & 0.4 & 0.0 & 2.1 \\
4.0 & 55.5 & 3.6 & 2.4 & 0.4 & 2.6 \\
4.4 & 55.5 & 4.5 & 3.2 & 0.8 & 3.0 \\
4.8 & 55.5 & 6.8 & 5.3 & 2.1 & 3.4 \\
5.2 & 55.6 & 9.9 & 8.2 & 5.0 & 3.9 \\
5.8 & 55.6 & 14.6 & 13.0 & 10.9 & 4.6 \\
6.6 & 55.7 & 21.6 & 19.7 & 17.3 & 5.5 \\
7.0 & 55.7 & 26.3 & 24.4 & 22.0 & 6.0 \\
7.5 & 55.8 & 31.7 & 29.9 & 27.0 & 6.5 \\
8.1 & 55.9 & 38.2 & 36.4 & 33.0 & 7.1 \\
8.8 & 56.0 & 46.6 & 44.8 & 40.9 & 7.8 \\
9.6 & 56.1 & 57.6 & 55.8 & 51.0 & 8.6 \\
10.6 & 56.3 & 72.3 & 70.7 & 64.7 & 9.6 \\
11.8 & 56.4 & 89.8 & 88.0 & 80.2 & 10.3 \\
12.5 & 56.5 & 97.3 & 95.3 & 86.5 & 10.4 \\
13.3 & 56.6 & 106.1 & 104.0 & 93.8 & 10.7 \\
14.2 & 56.7 & 111.4 & 109.2 & 98.1 & 10.6 \\
15.3 & 56.7 & 109.3 & 107.1 & 96.4 & 10.3 \\
\hline
\end{tabular}

Table 2: Turbine Performance Computed from CFD.

\begin{tabular}{|c|c|c|}
\hline Wind Speed $(\mathrm{m} / \mathrm{s})$ & Rotor Speed $(\mathrm{rpm})$ & $\begin{array}{c}\text { Computed Aerodynamic } \\
\text { Power }(\mathrm{kW})\end{array}$ \\
\hline $\mathbf{8 . 8}$ & $\mathbf{5 6 . 0}$ & $\mathbf{5 3 . 9}$ \\
\hline
\end{tabular}

Final Report

23

CRAFTR-08.2011.030

Prime Contract No. DOE-DE-EE0003269

Combustion Research and Flow Technology, Inc. 


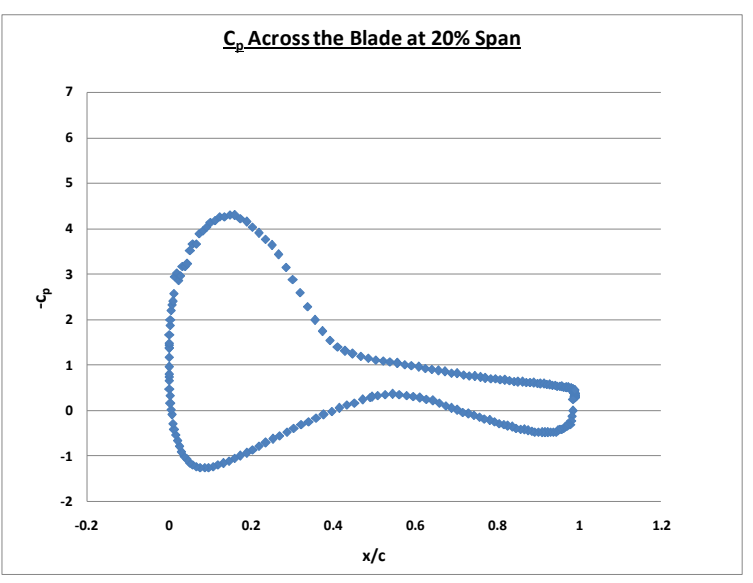

$20 \%$ Span

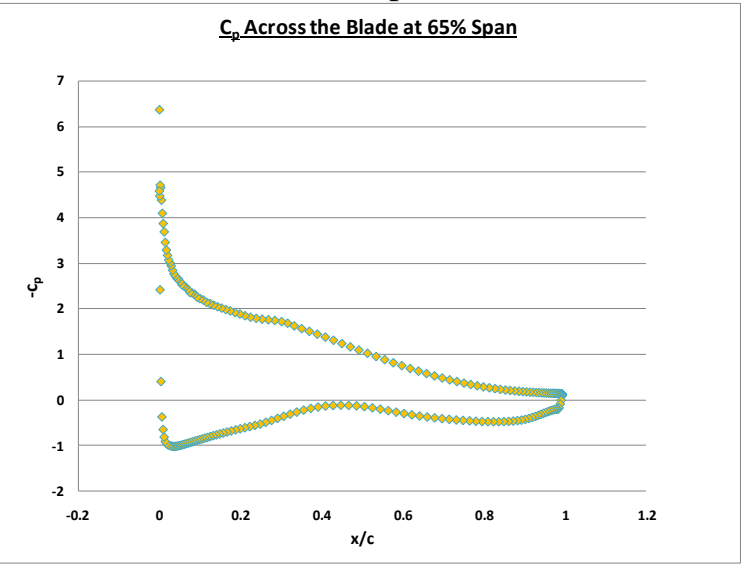

$65 \%$ Span

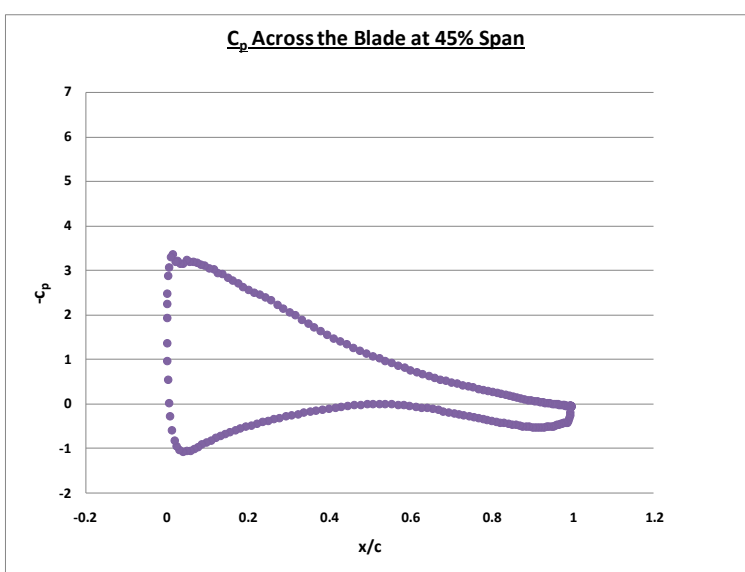

$45 \%$ span

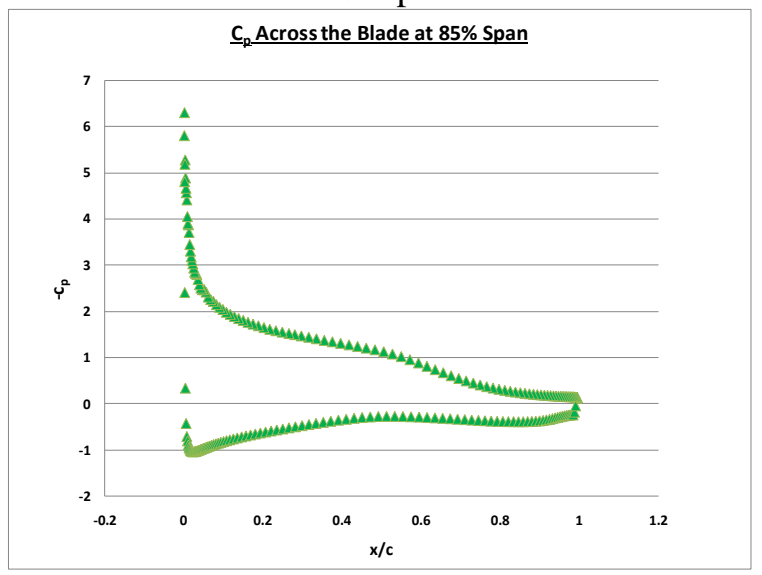

$85 \%$ Span

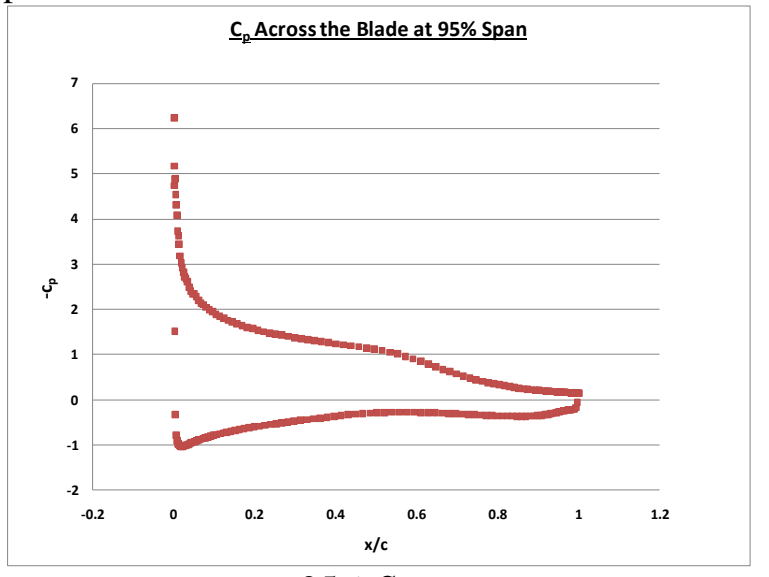

$95 \%$ Span

Figure 25: Chordwise Pressure Distribution at 5 Spanwise Locations $(20 \%, 45 \%, 65 \%, 85 \%$ $95 \%)$.

Final Report

24

CRAFTR-08.2011.030

Prime Contract No. DOE-DE-EE0003269

Combustion Research and Flow Technology, Inc. 


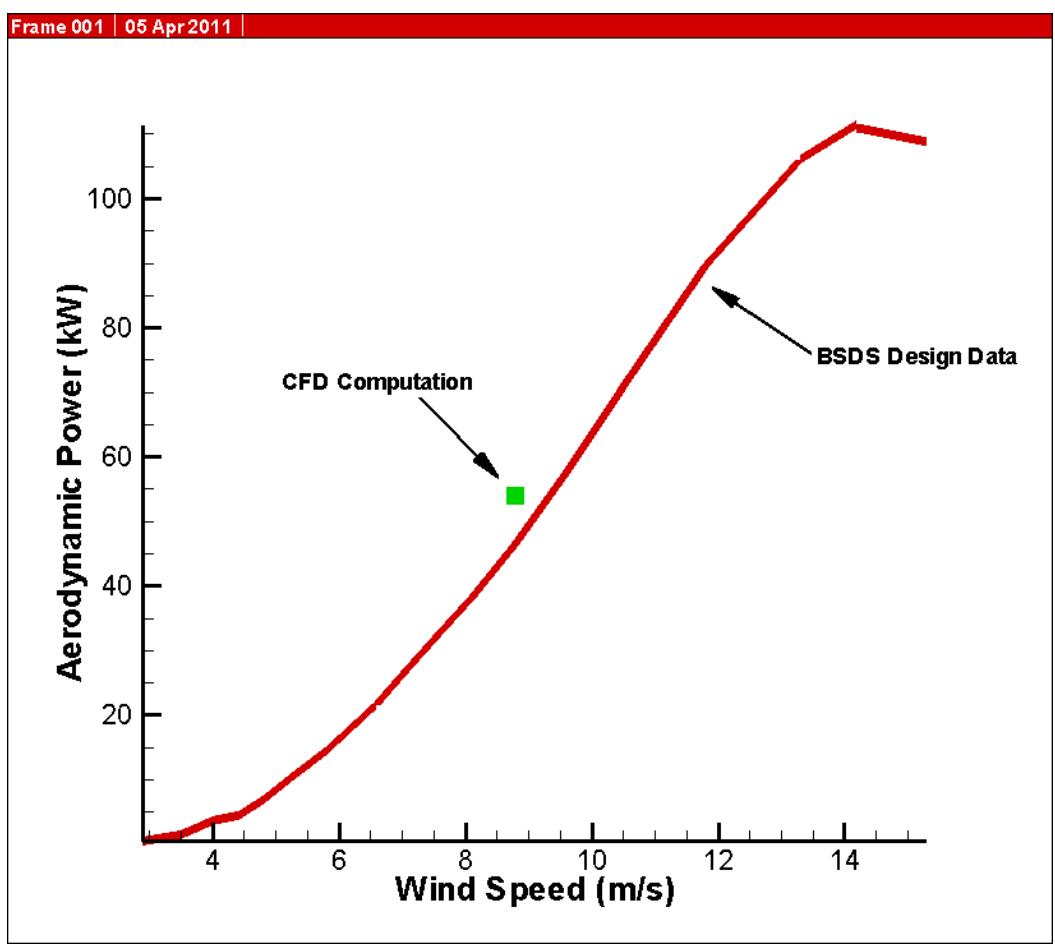

Figure 26: Comparison of Turbine Performance for the CFD Computation with Design Data [2].

\subsubsection{Tip Vortex Simulation}

One of the primary goals of this program is to reduce noise associated with the operation of wind turbines and develop diagnostic and computational tools that aid in achieving this end. In the next few sections we will discuss tonal noise due to the rotation of the turbine blades, which includes thickness and loading noise associated with the rotor blade. In this section we utilized CFD computations to capture the tip vortex that is generated from the operation of the wind turbine. Accurately capturing the tip vortex is important because vortex shedding from the trailing edge of the rotor blade is an important source of noise. The wind turbine design that has been used for the simulation of the tip vortex is the BSDS blade that was developed by the Sandia National Laboratories Blade Systems Design study [2] and has been discussed in the previous section. The wind turbine has 3 blades and a computational domain comprising of a single blade embedded in a 120 degree wedge was utilized for this simulation. The wind speed in

Final Report 
this case was $8.8 \mathrm{~m} / \mathrm{s}$ and the ambient pressure was assumed to be $101325 \mathrm{~Pa}$. The wind turbine had a 1 degree pitch at the tip and based on the design data was rotating with an rpm of 56.

Most of the work involved in the tip-vortex simulation was expended in the grid generation process. As mentioned in Section 3.3.2, the CRUNCH CFD ${ }^{\circledR}$ code, is a multielement solver that utilizes a combination of tetrahedral, prismatic, pyramidal and hexahedral cells to efficiently solve flows in complex systems. In the case of the BSDS blade, the geometry exhibits considerable complexity - for example the blade profile has a circular shape at the root that transforms into a more conventional airfoil shape section at the tip; the chord also initially elongates as we move from root to tip and then contracts; furthermore the blade has a 12 degree twist from root to tip. The surface grid is shown in Figure 27 where both the root and tip are resolved with hexahedral meshes to resolve the large gradients that are exhibited in these regions due to the boundary layer and vorticity respectively. Figure 28 shows the highly resolved tip vortex region extending out azimuthally comprising primarily of high aspect ratio hexahedral cells that are necessary to capture the large gradients in velocity. It should be noted that away from the tip and the blade the grid topology transitions to large tetrahedral cells that were necessary to minimize the overall size of the grid. The resulting tip vortex from the simulation is shown in Figure 29 where an iso-surface of vorticity is plotted. Apart from the well defined vortical structures associated with the blade tip, there is also some production associated with the blade due to its shape and the twist in the blade.

Final Report 


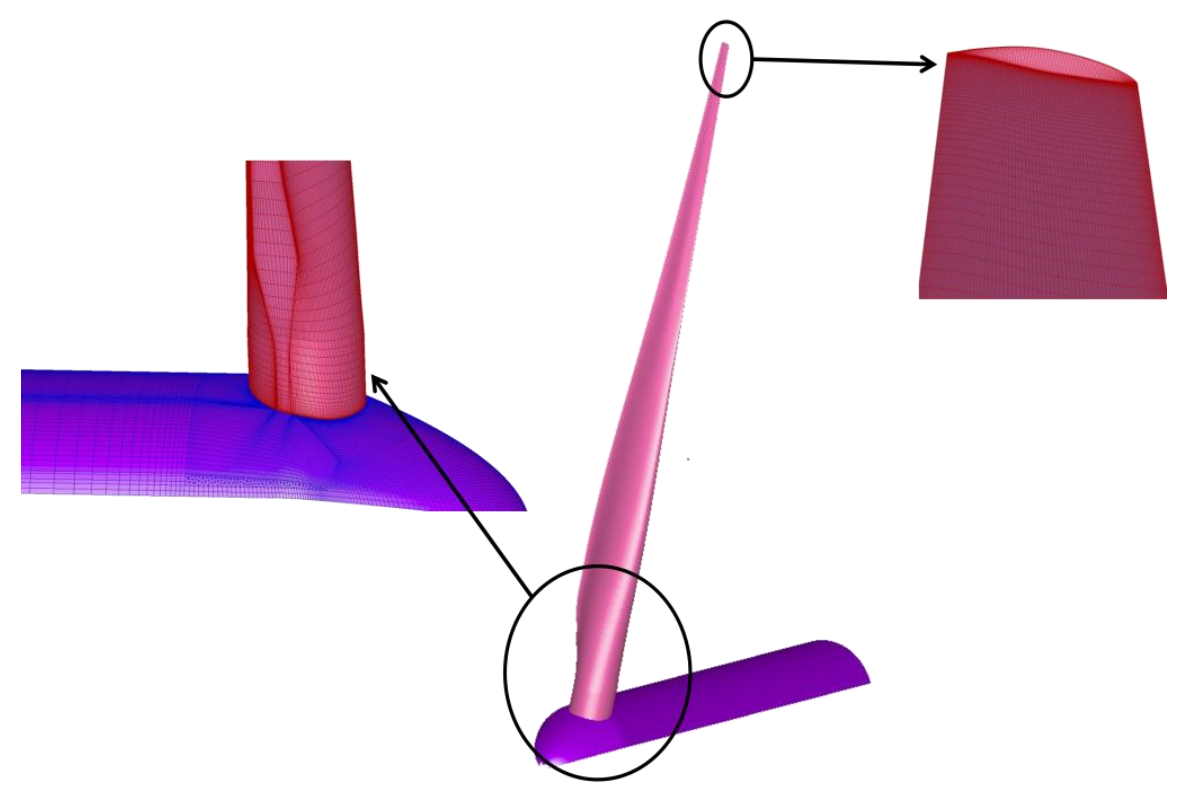

Figure 27: Surface Grid near the Root and Tip of the Blade.

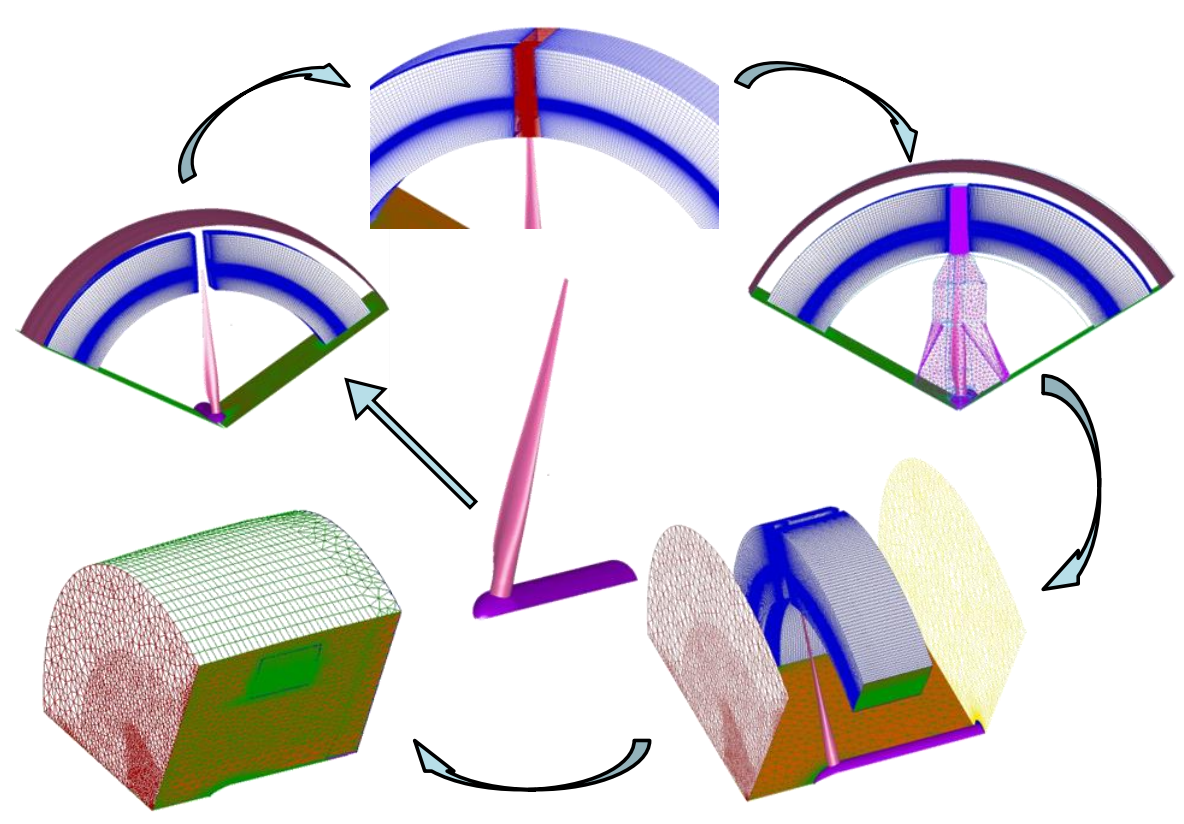

Figure 28: High Resolution Grid to Resolve the Tip Vortex.

Final Report

Prime Contract No. DOE-DE-EE0003269

Combustion Research and Flow Technology, Inc. 


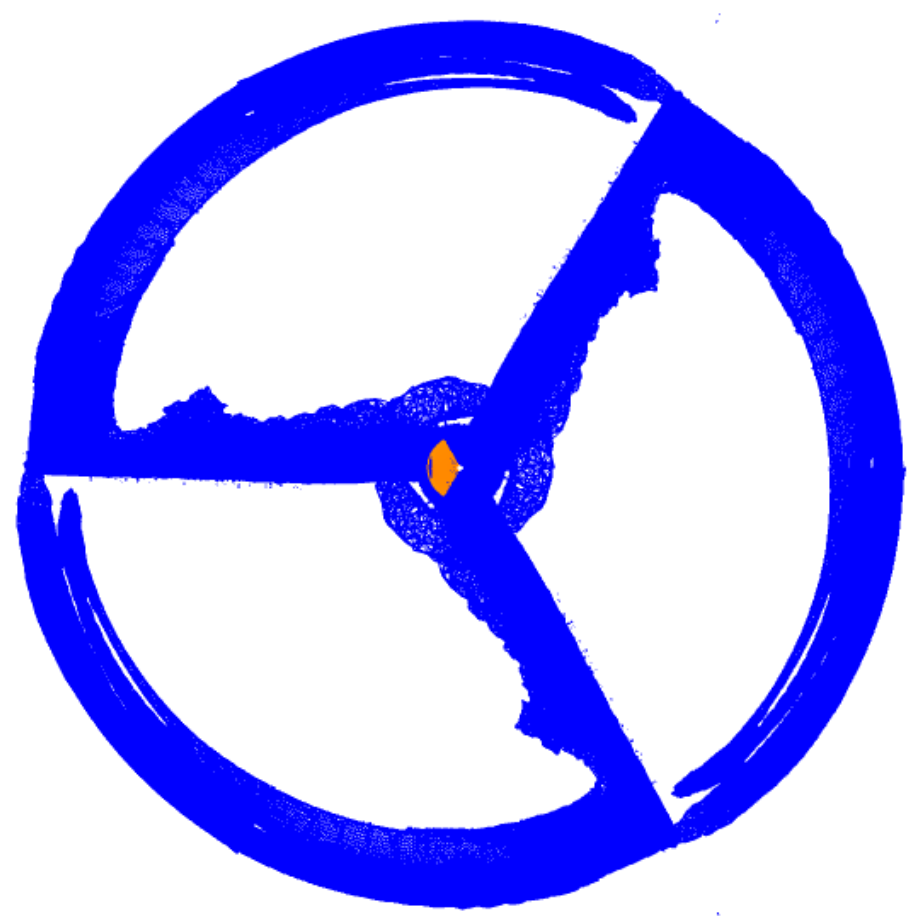

Figure 29: Iso-surface of Vorticity showing Tip Vortex and vorticity production around the blades.

\subsubsection{Acoustic Solver}

CRAFT Tech has developed an Acoustics toolkit for the prediction of far-field noise for aerospace applications that includes aircraft, jets, wakes, fans, propellers, helicopter rotors, etc. The toolkit utilizes steady and/or unsteady high-fidelity CFD solutions along with far-field noise propagation and prediction methods such as the Kirchhoff method and the Ffowcs-Williams and Hawkings (FW-H) equation [5]. In most applications the time history of the flow variables is recorded on the Acoustic Data Surface. However, for turbomachinery problems, this is also analogous to a steady loading on a blade surface rotating with the blade passage frequency. In the example shown below, we extract the steady blade loading from the CFD solution for a fan/impeller. Utilizing the Ffowcs-Williams and Hawkings equation we can compute the noise radiated to a far-field location [6]. The source of the noise radiation is the result of (i) the monopole term which is attributed to the displacement of fluid due to the rotation of the blade and (ii) the dipole term due to the rotation of a steady loading on the blade.

Final Report 

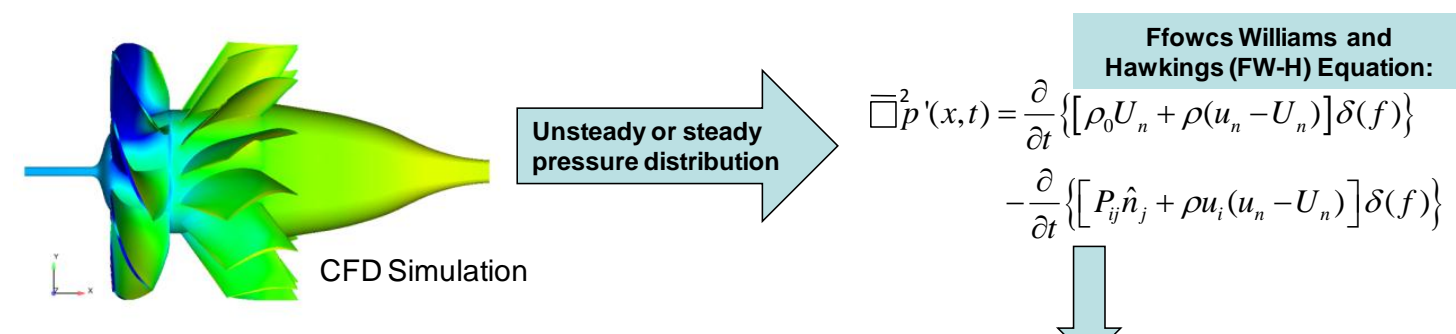

Farassat Formulation $1 A$ of $\mathrm{FW}-\mathrm{H}$ :

$$
\begin{aligned}
4 \pi p_{D}^{\prime}(\vec{x}, t) & =\frac{1}{c_{0}}\left\{\int_{f=0}\left[\frac{\dot{L}_{r}}{r\left(1-M_{r}\right)^{2}}\right]_{r e t} d S+\int_{f=0}\left[L_{r} \frac{r \dot{M}_{r}+c\left(M_{r}-M^{2}\right)}{r^{2}\left(1-M_{r}\right)^{3}}\right]_{r e t} d S\right\} & 4 \pi p^{\prime}{ }_{M}(\vec{x}, t)=\int_{f=0}\left[\frac{\rho_{0}\left(\dot{U}_{n}+U_{\dot{r}}\right)}{r\left(1-M_{r}\right)^{2}}\right]_{r e t} d S \\
& +\int_{f=0}\left[\frac{L_{r}-L_{M}}{r^{2}\left(1-M_{r}\right)^{2}}\right]_{r e t} d S & +\int_{f=0}\left[\frac{\rho_{0} U_{n}\left(r \dot{M}_{r}+c\left(M_{r}-M^{2}\right)\right)}{r^{2}\left(1-M_{r}\right)^{3}}\right]_{r e t} d S
\end{aligned}
$$

Monopole term: geometry and motion of

Dipole term: unsteady loading on blade surface or rotational steady loading rotating blade

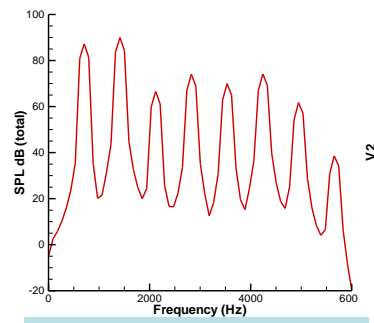

SPL Spectrum at observer

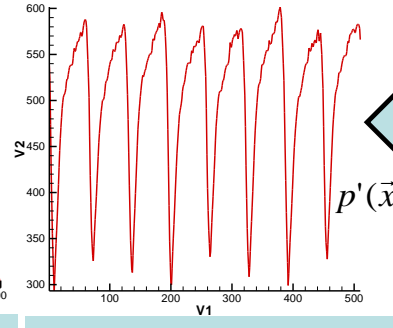

Pressure time history at observer

far-field acoustic solve

\section{Figure 30: Acoustic Toolkit used to Predict Noise from CFD Solutions of Rotating} Machinery.

For our application of the Acoustic toolkit to wind turbine noise, we compute the noise in the far-field from the wind turbine blades utilizing this FW-H equation methodology. Figure 31 shows a simplified schematic of the problem solving process. The Acoustic Data Surfaces (ADS) from which the far-field noise is computed encompasses the blade and rotates about the turbine hub axis with the blades. Our implementation of the FW-H Equation allows for the subsonic motion of the acoustic data surfaces relative to the observer. The major assumption here, for the moment is that scattering from the ground plane will not be accounted for. This may be reasonable since the ground scattering effects will impact all designs and only the relative noise trends are important from a design perspective.

Final Report

Prime Contract No. DOE-DE-EE0003269

Combustion Research and Flow Technology, Inc. 


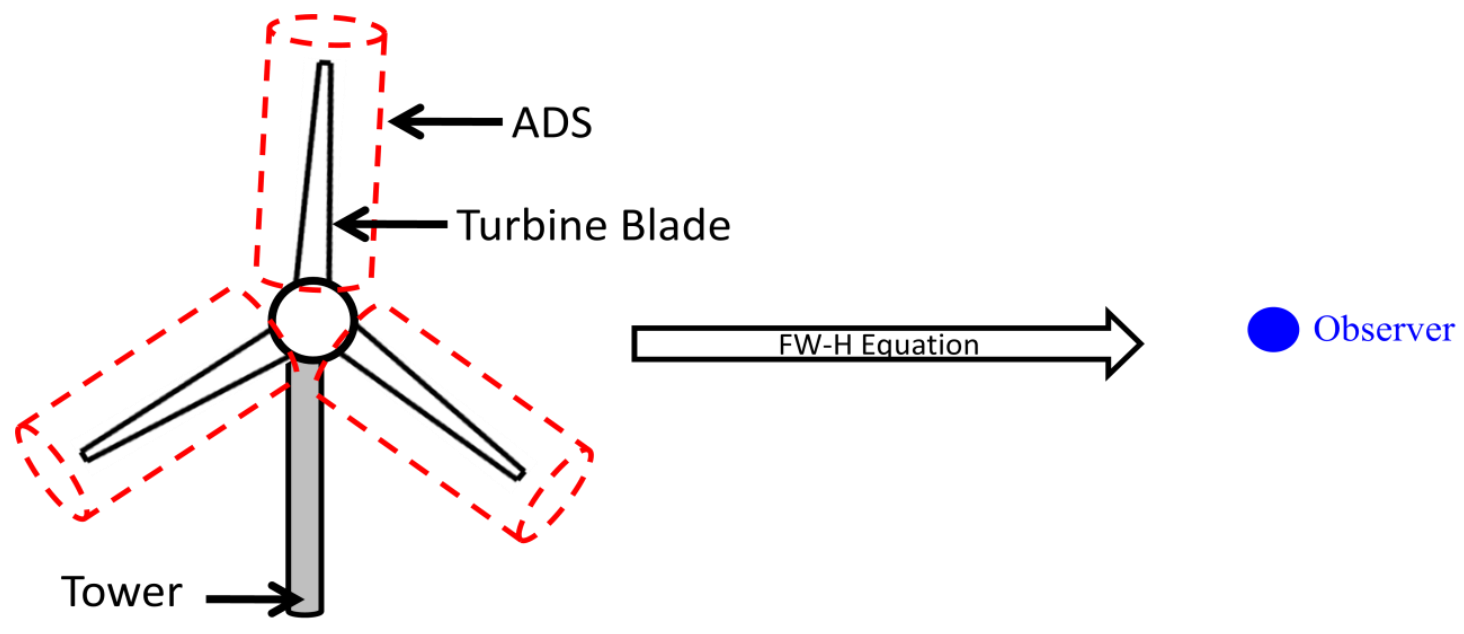

Figure 31: Schematic of Framework for Predicting Noise from Wind Turbines including Ground Effects.

\subsubsection{Acoustic Predictions}

The results of the CFD computations discussed in Section 3.3.3 were used for the prediction of noise utilizing the procedure detailed above. For our noise calculations, the microphone array is assumed to be located in a circular arc at a radius of $18 \mathrm{~m}$ away from center of the wind turbine (See Figure 32). A polar plot of the overall sound pressure levels due to the rotation of the turbine blades is provided in Figure 33. The plot shows that the peak noise levels of $75 \mathrm{~dB}$ are along the 60, 120, 240 and 300 degree angular directions. The large twist in the blade is partially responsible for the angular lobes. Again it should be stressed that these noise levels are primarily due to the rotation effect (spinning dipoles) of the wind turbine and have been extracted from the steady CFD computations. The other noise generating mechanisms related to tip vortex noise and trailing edge shedding instability are beyond the scope of the current work and will be studied in the future.

Final Report 


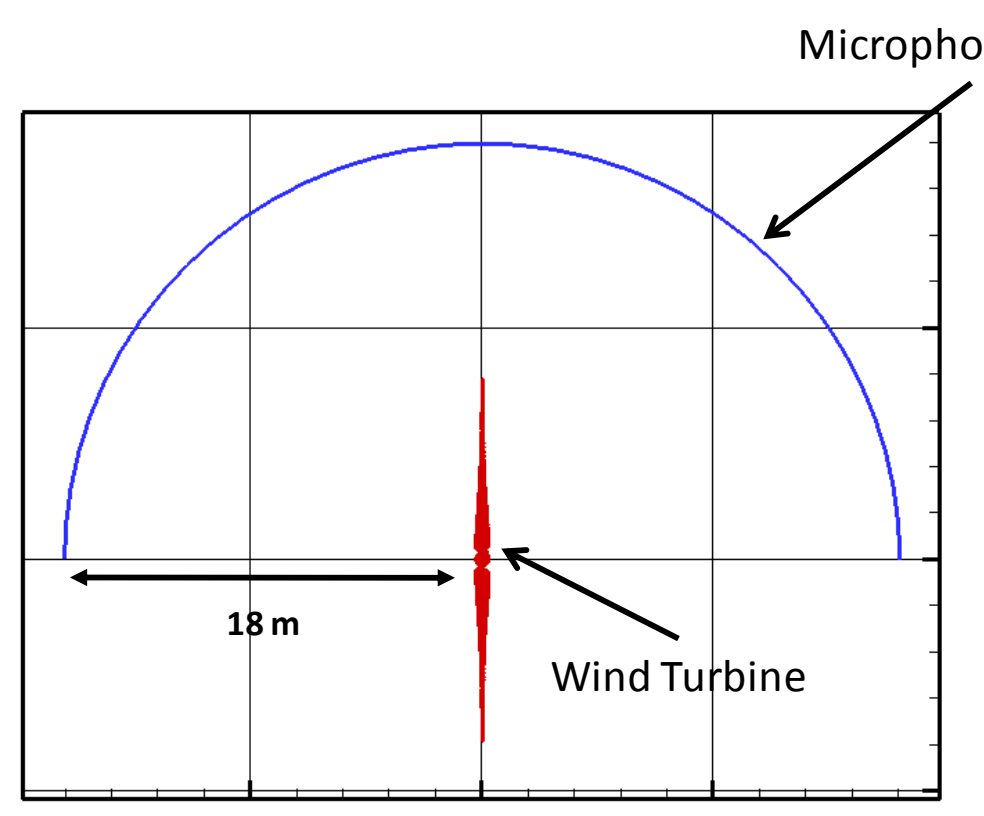

Figure 32: Schematic showing location of microphones for predicting noise from the operation of wind turbine.

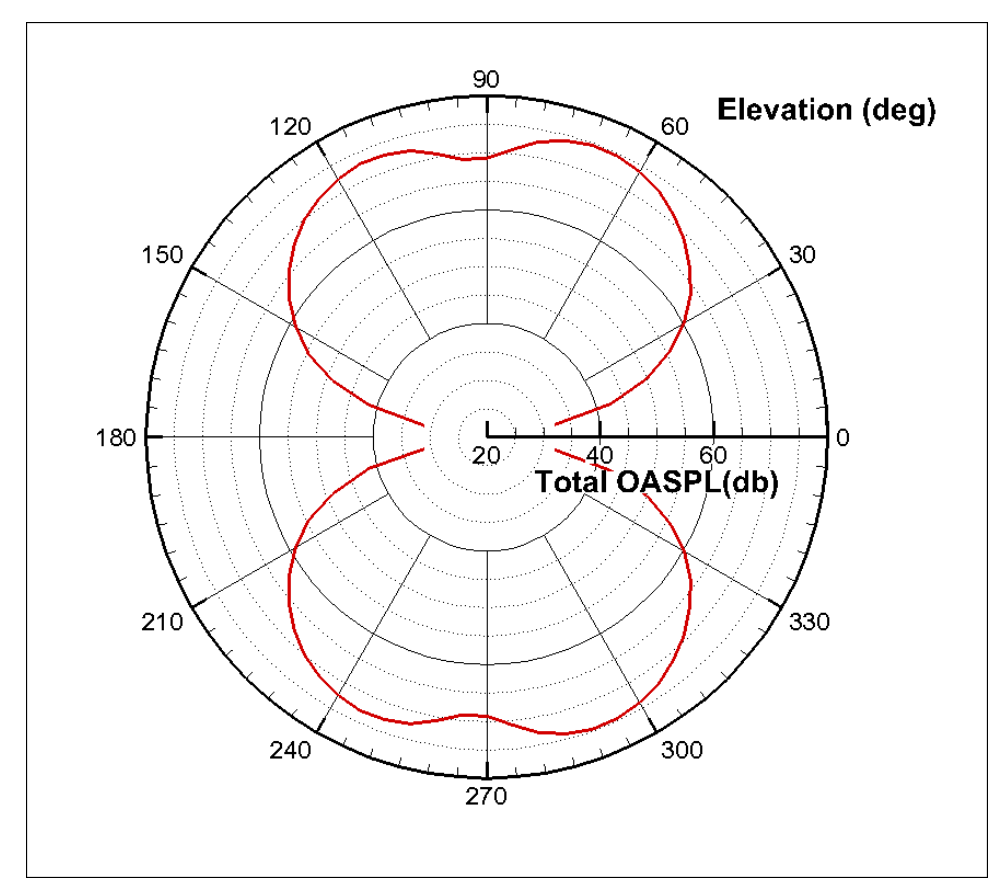

Figure 33: Polar Plot of Overall Sound Pressure Levels (SPL) generated by the rotary motion of the wind turbine blades.

Final Report 


\subsection{Design Optimization Study}

In this activity, we are involved in development of a design optimization toolkit to optimize the shape of wind turbine blades. Our focus is on the design of wind turbine blades optimized for improving aerodynamic performance and reducing noise. The framework that we have constructed is general and can be extended to include aero-elastic effects and structural analysis. In the next sub-section, we will discuss the Design Optimization Toolkit and the different sub-components that have been used to construct the toolkit. This will be followed by a short discussion on the parameterization procedure and definition of the design variables selected for our optimization studies. Lastly, we will discuss results of the design optimization study.

\subsubsection{Design Optimization Framework}

CRAFT Tech has developed a shape optimization toolkit (See Figure 34) that combines modules such as a stochastic optimization algorithm with a shape deformation generator (SCULPTOR) or a grid generator with a high-fidelity flow solver (CRUNCH CFD ${ }^{\circledR}$ ). The stochastic optimization technique used has been based on an evolutionary algorithm such as a genetic algorithm. Such stochastic optimization methods are attractive because they search the design landscape in a global manner and are particularly effective in non-linear and noisy landscapes defined by discontinuous and/or multi-modal functions. Shape optimization is carried out by parameterization of the geometry of interest with design variables. For complex 3-D shapes such as those seen in wind turbine applications, a series of control points are established that define the turbine blade through a network of Bezier curves. The control points are used as design variables for shape optimization, and shape change is smooth, as control points describe higher derivatives of shape contours in parametric space. Once the turbine blade is parameterized by SCULPTOR and the control points established as design variables, the optimization loop is initiated. The design variables are passed by the optimization algorithm to SCULPTOR where the control points are altered based on the design parameters and the shape of the design is modified. The computational grid is then passed to the CFD code which is used to carry out a RANS simulation. The two fitness functions for (i) aerodynamic performance and

(ii) noise are evaluated from the CFD solution and passed back to the evolutionary algorithm which starts the design iteration all over again.

Final Report 


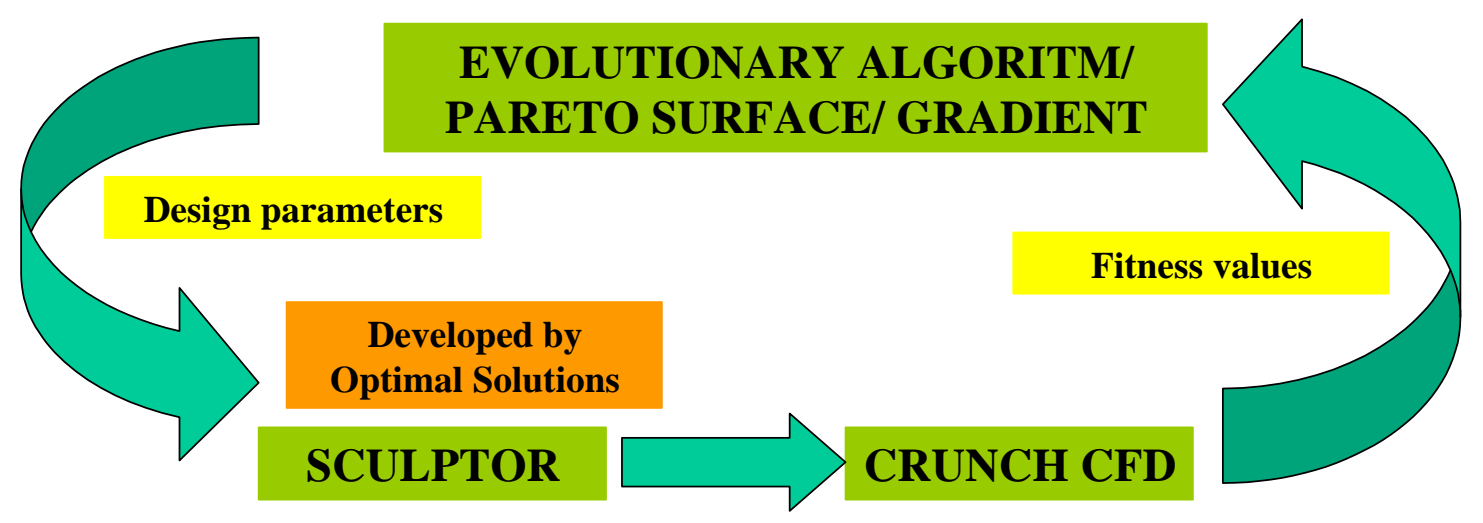

Figure 34: Schematic showing Flow of Information in Design Optimization Toolkit.

Our shape optimization procedure has been used for design optimization studies representative of fluid dynamic flowfields with complex physical phenomena such as multielement airfoils that serve as control surfaces on submarines [8], cavitating airfoils for cryogenic turbopumps, nozzles for noise reduction, combustors for scramjet engines and lift fans for hovercrafts [9].

\subsubsection{Optimization Algorithm}

In our optimization toolkit we include two optimization methodologies: Genetic Algorithms (GA) and Kriging Surface Methodology with a Surrogate Model. We commonly use GA with the Kriging Surface used for complex MDO problems. For the sake of brevity, we will discuss only our GA implementation here. GA's are based on the principles of Darwin's theory of evolution and natural selection. The key ideas of how design unfolds in nature in an efficient, parallel and multi-modular manner satisfying a complex network of constraints, variables and objectives are embodied in the workings of GAs. Formal presentation of the ideology is based on seminal work of Holland [10] that structures based on chromosome-like string of binary switches could trigger more favorable characteristics in systems if the chromosomes were permitted to interact with other similar structures based on some measure of fitness, thereby, reproduce and mutate leading to offspring systems that were better adapted to the environment. Efficient combinatorial procedures with operators for selection, crossover and mutation are used to advance or evolve a design by the evolutionary algorithm. These operators are standard in the

Final Report 
Genetic Algorithm literature although many advanced variants of these operators exist and in our particular implementation we utilize tournament selection with elitism and uniform (multi-point) crossover [12] to speed up convergence. In our implementation of the GA, the chromosomes utilize binary encoding. Although many engineering applications use real coded chromosomes, the lower cardinality binary encoding replicates evolutionary processes closely (for example, four types of nucleotide encode an alphabet in the human genome). Fundamental GA theory also postulates that alphabets of lower cardinality provide greater flexibility during crossover and are not blocked in their search for a global extreme in certain complex landscapes [11]. Convergence can also be accelerated by the preservation of diversity in the design population and it is ensured by niching or fitness sharing by sub-populations that define a certain niche in a multi-modal design space. In particular, following the lead of Carroll [12], we have used Goldberg's multi-dimensional phenotypic sharing scheme with a triangular sharing function. For multi-objective optimization problems we follow the ranking scheme of Fonseca and Fleming [13] in obtaining the Pareto optimal set of solutions, by assigning ranks to the individuals in the population based on their relative domination.

\subsubsection{Parameterization and Design Variables}

Shape parameterization and deformation is achieved by specifying the shape design utilizing the shape deformation tool SCULPTOR that has been developed by Optimal Solutions. The complex shapes are defined by a network of control points (Figure 35). The control points are specified as design variables and their displacement results in smooth variation of the boundary, since the control points influence the displacement of a series of surface nodes. In SCULPTOR a grid of control points is constructed (known as an Arbitrary Shape Deformation grid) around the geometry. For CFD applications, the control points of the ASD grid influence the movement of grid points on the CFD grid. The movement of the grid points, in turn influences the displacement of the boundary (Figure 36(c)).

Final Report 


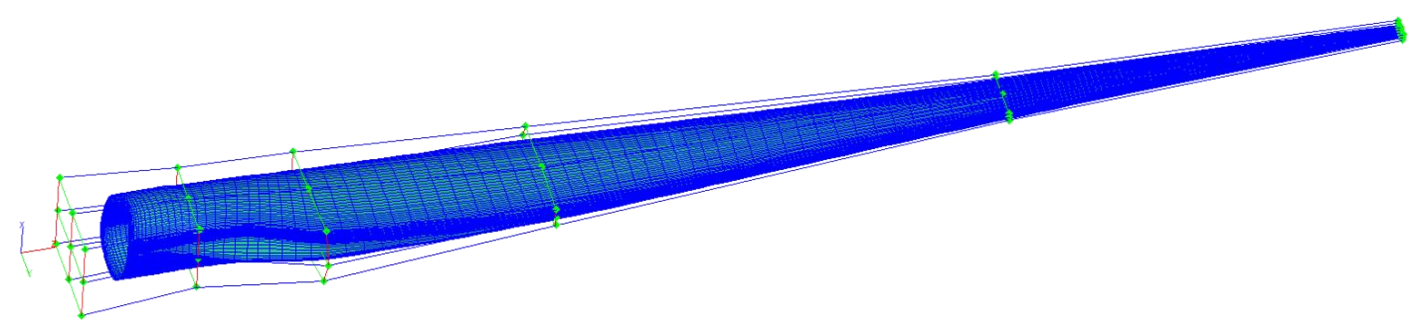

Figure 35: Shape Deformation Grid produced by SCULPTOR and ASD Grid Shown by red, green and blue lines with control points as nodes on the grid.

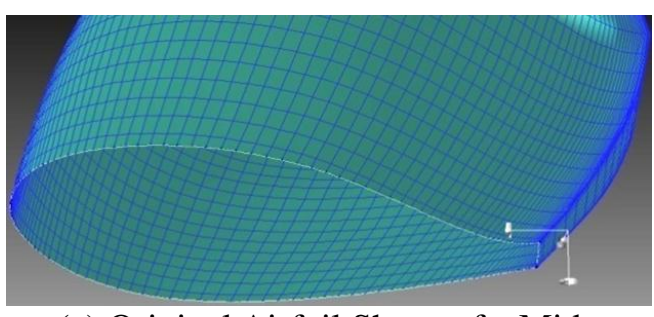

(a) Original Airfoil Shape of a MidSection with surface grid.

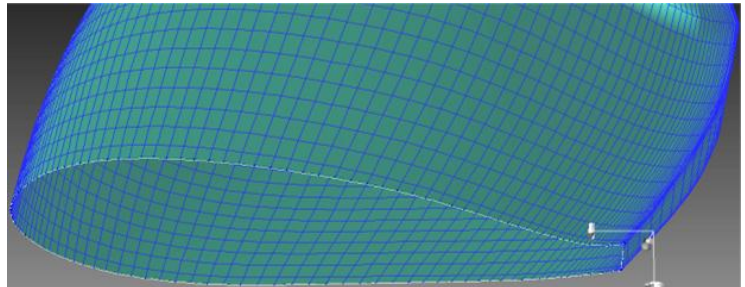

(b) Sample Modified Mid-section Blade geometry with reduced thickness retaining the flatback shape. Shape generated by moving control points .

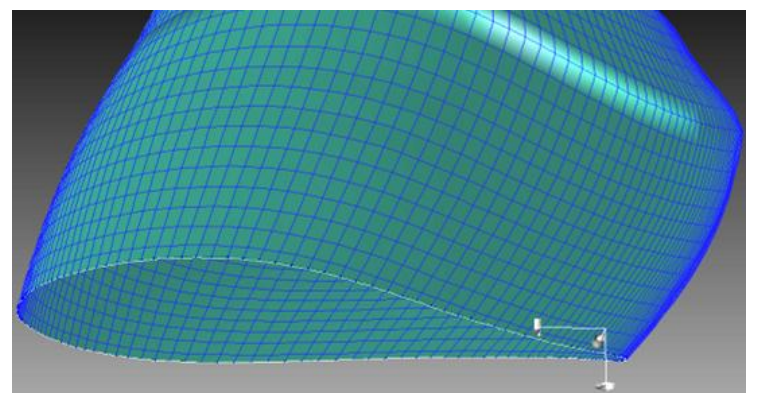

(c) Blade geometry of mid-section deformed by sculptor showing smoothly varying contour with a sharp trailing edge.

Figure 36: Shape modifications to the turbine blade generated by moving the control points.

This methodology of shape deformation is very powerful for multiple reasons: (i) it ensures smooth design shapes (ii) the grid on the modified design is the same as the original grid in terms of the cell topology and cell/node count (iii) constraints related to the shape can be easily defined (iv) the final design can be ported to CAD (v) flexibility in defining the number of

Final Report 
control points/design variables (v) feasibility of affecting large changes to the geometry which works well in conjunction with global optimization methods.

In our optimization study, the deformation of the wind turbine blade geometry was defined by control points spread across airfoil cross-sections at 5 planes (See Figure 37). The shape of each airfoil section was defined by 6 control points (design variables), 3 of which define the airfoil shape on the pressure side (pressure_leading, pressure_center, pressure_trailing), while the remaining 3 define the airfoil shape on the suction side (suction_leading, suction_center, suction_trailing). Movement of these control points alters the surrounding grid and the shape of the airfoil contour. The displacement of each control point is independent of the other control points on a given airfoil section. However, the movement of a given control point is weighted across the different cross-sections. For example, the pressure_leading control point is given $100 \%$ of the defined displacement at the first cross-section and only $3 \%$ of the displacement at the tip. This weighting dependency ensures a smooth variation in airfoil profiles along the span of the blade. More importantly, it permits shape deformation of the entire wind turbine blade with just 6 variables leading to a large savings in the design optimization process.

Final Report 

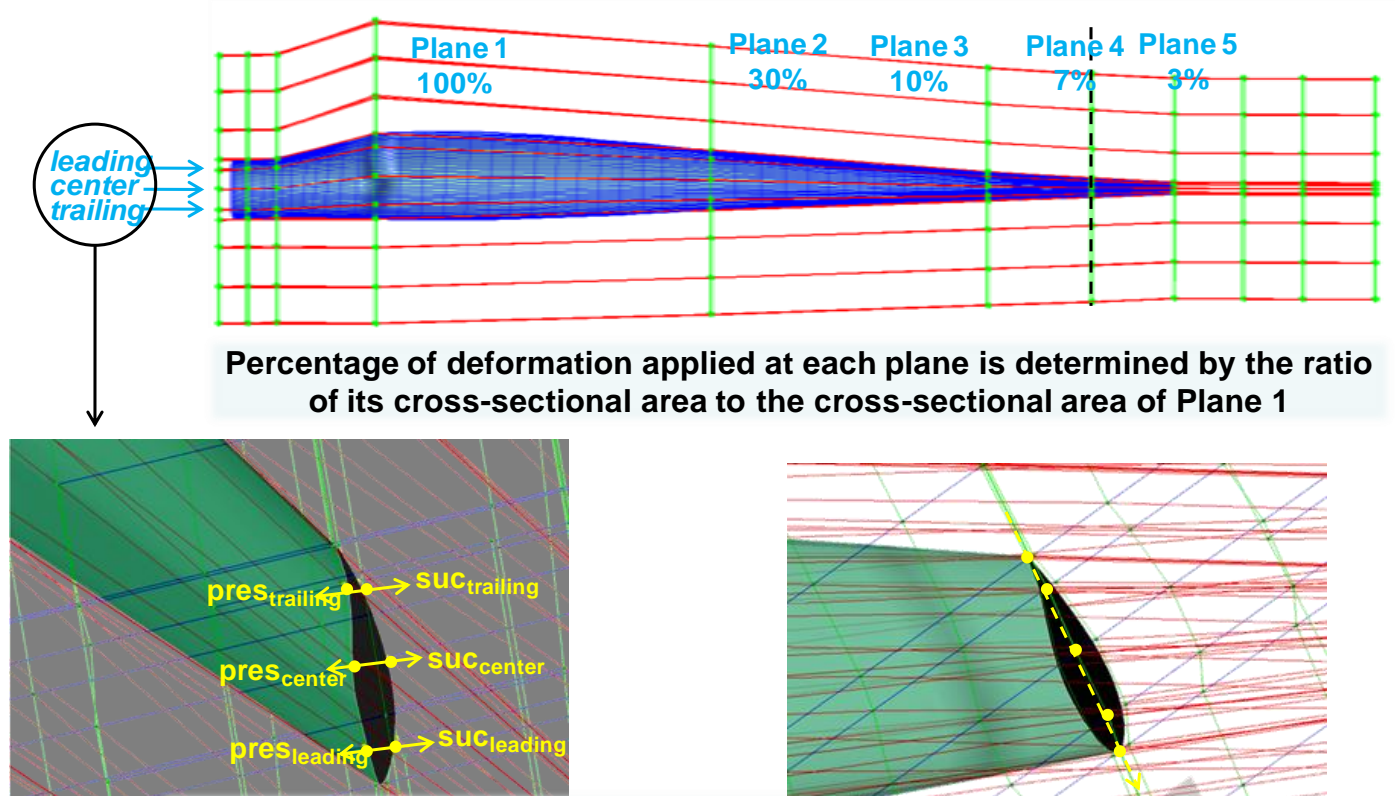

Six Design Variables:

- Pressure leading $_{\text {Pressure }}$ center

Pressure $_{\text {trailing }}$

- Suction leading Suction $_{\text {center }}$

Suction trailing

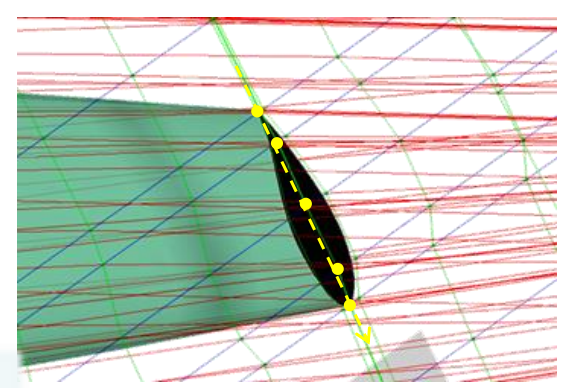

Fixed plane along the centerline isolates the deformations along the pressure side and suction side

Figure 37: Parameterization of Wind Turbine Blade Geometry for Design Optimization Study.

\subsubsection{Design Optimization Results}

Shape optimization of the wind turbine blade is discussed in this section. As mentioned earlier, the baseline shape of the wind turbine was the BSDS blade shape. The primary consideration for this demonstration design optimization exercise is tonal noise reduction. However, tonal noise is directly dependent on blade loading. Therefore, it is more appropriate to carry the design optimization exercise by improving the aerodynamic performance and reducing the noise simultaneously. Both objectives, are in a sense, conflicting, since in most cases increasing the blade loading leads to increased noise and vice versa. Furthermore, it should be mentioned that the baseline BSDS design is an optimized design in terms of aerodynamic performance and structural integrity and any improvement would be a significant achievement. The objective function used for our design optimization study was defined as the ratio of the aerodynamic power to the rms of pressure (peak levels) or in other words the aerodynamic

Final Report 
power per unit of sound pressure level. This definition would facilitate improved designs for blade loading that generated less noise. As mentioned in the previous section 6 design variables were used to define the variation in shape of the wind turbine blade. A population of 8 designs was considered for the genetic algorithm based optimization procedure. The optimization process was carried out utilizing the design optimization framework discussed in Section 3.4.1. The GA based optimizer provided the design variables to SCULPTOR which was used to deform the geometry and computational grid. Steady CFD computations were performed to get an estimate of the aerodynamic power, and the steady solution was passed to the acoustic solver (Section 3.3.5) to estimate the tonal noise levels from the steady blade loading.

A phase space map of all the designs analyzed is shown in Figure 38 where the nondimensional aerodynamic power level and the sound pressure level for each design is shown. The designs that lie along the Pareto-front represent designs that are non-dominated i.e. those designs that are not dominated by both sound pressure levels and aerodynamic power by the rest of the designs in the design landscape. There are three designs that represent interesting features in the design space and they are marked in Figure 38 by their GA designation (G15D1, G15D3 and G14D1). G15D1 represents a design that generates the maximum amount of power. Consequently, it also has significantly higher tonal noise. However, it is noteworthy because tweaking the BSDS design can lead to approximately $20 \%$ more aerodynamic power which is extremely attractive for designers of wind turbines. G14D1 represents a design that generates roughly 5\% more power than the BSDS blade but also generates less tonal noise. Design G15D3, on the other hand generates roughly the same amount of power as the BSDS design but about $0.6 \mathrm{~dB}$ less noise than the BSDS blade. In locations where noise is a greater concern for the operation of wind turbines, the designs to the left of G15D3 along the Pareto-front generate less noise at the cost of aerodynamic power. For example there is a design that generates only 72 $\mathrm{dB}$ noise, which is over $2.5 \mathrm{~dB}$ lower than the BSDS design. However, this comes at a huge cost of the aerodynamic power levels.

Final Report 


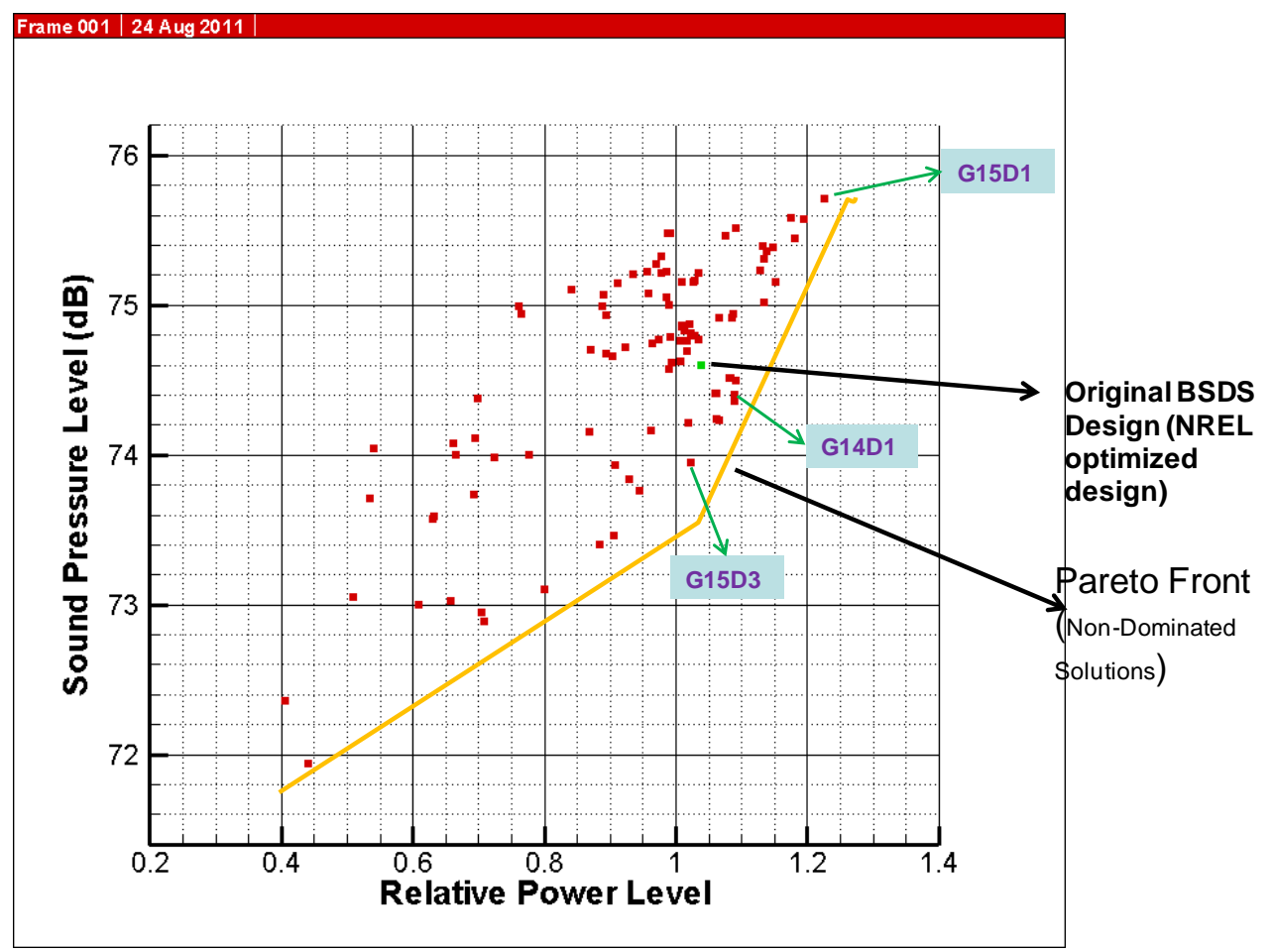

Figure 38: Design Landscape showing Power Generated by each design and its noise characteristics. The design landscape also shows a subset of designs (Pareto-Front) where designs having the non-dominated solutions exist.

A comparison of the different blade cross-sections and the cross-sections of the abovementioned three designs is shown in Figure 39. G15D1, the design with the maximum power, not surprisingly also shows increased thickness throughout the span. This leads to higher pressures on the pressure side of the airfoil sections (Figure 40). However, the abrupt change in slope close to the trailing edge on the suction side leads to uneven pressure loading and may be responsible for the large increase in noise levels for this design. The G15D3 has a comparable blade section profile as the original BSDS blade with small differences in the shape of the airfoil sections closer to the hub (See Figure 39). As a consequence there are small differences in pressure between the BSDS blade sections and the G15D3 blade (See Figure 41). The G14D1 design is the most interesting since it provides greater power than the BSDS design and generates relatively less noise. As seen in Figure 42 it generates more lift at the mid-span section primarily due to higher loading on the pressure side of the airfoil sections. More interestingly, a

Final Report 
polar plot of the rms pressure distribution (Figure 43) indicates that for the primary lobe (about 75 degrees) the BSDS pressure fluctuation is larger than the G14D1 design. However, for the secondary lobe (around 110) the pressure fluctuation is slightly higher on the G14D1 design than the BSDS blade. Otherwise the directivity patterns on the all the designs is similar to the original BSDS design.

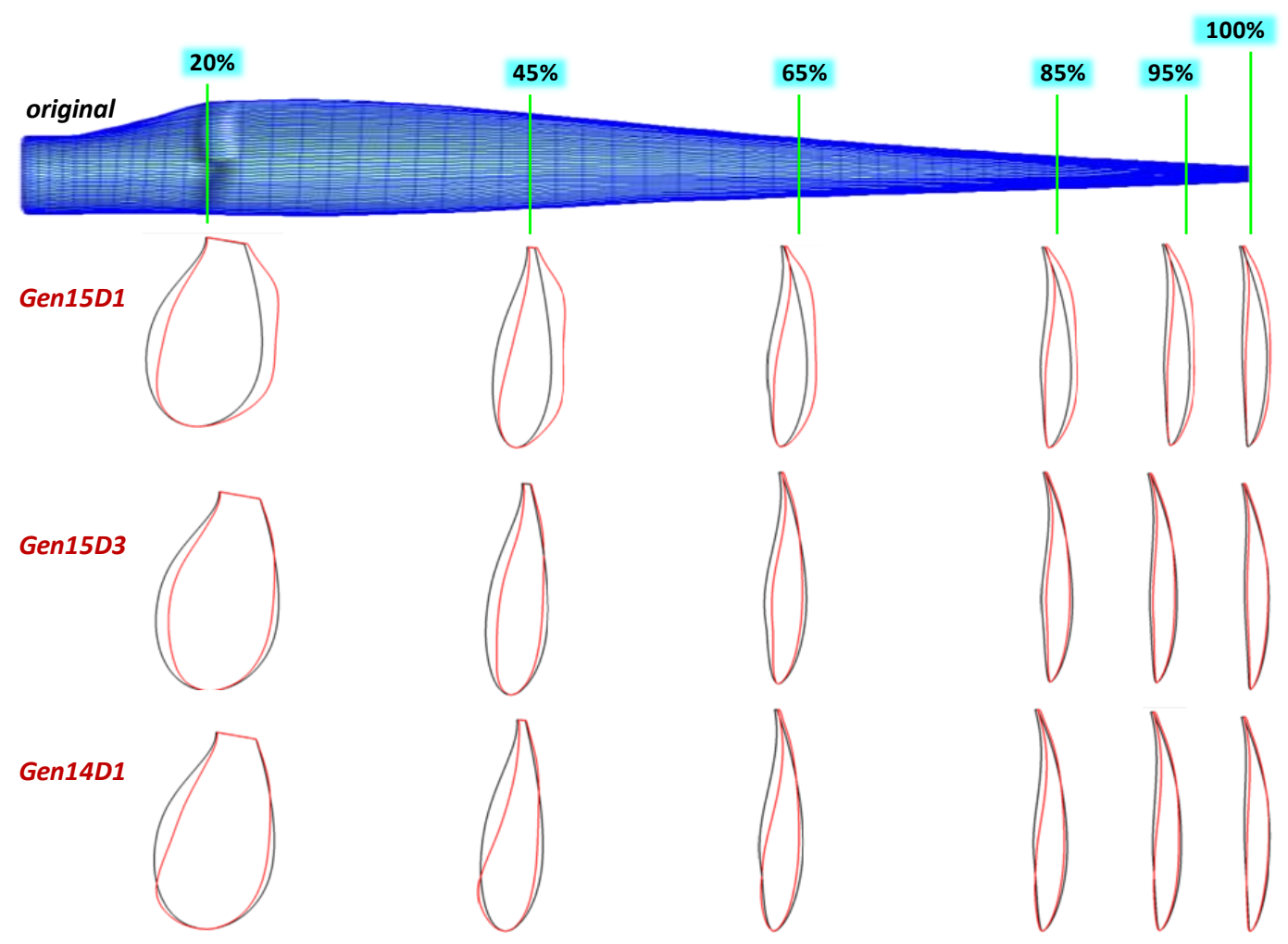

Figure 39: Airfoil Cross-Section Shapes for Different Designs Extracted from Design Space. Cross-Section of BSDS Shape is shown in Black. Other Designs are shown in Red.

Final Report 

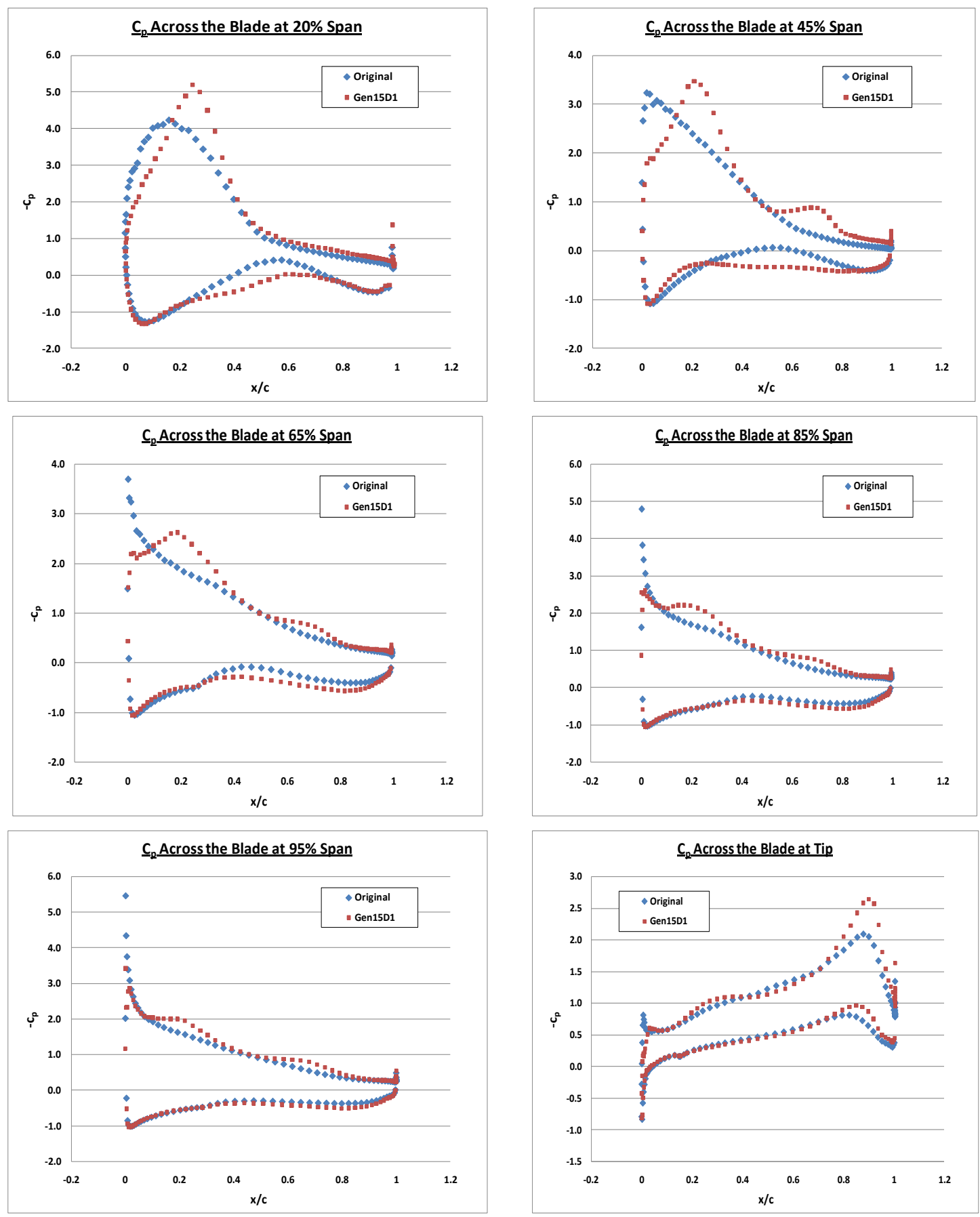

Figure 40: (-Cp) Comparisons of different cross-sections of the original BSDS wind turbine blade (blue) and the G15D1 design (red).

Final Report

Prime Contract No. DOE-DE-EE0003269

Combustion Research and Flow Technology, Inc. 

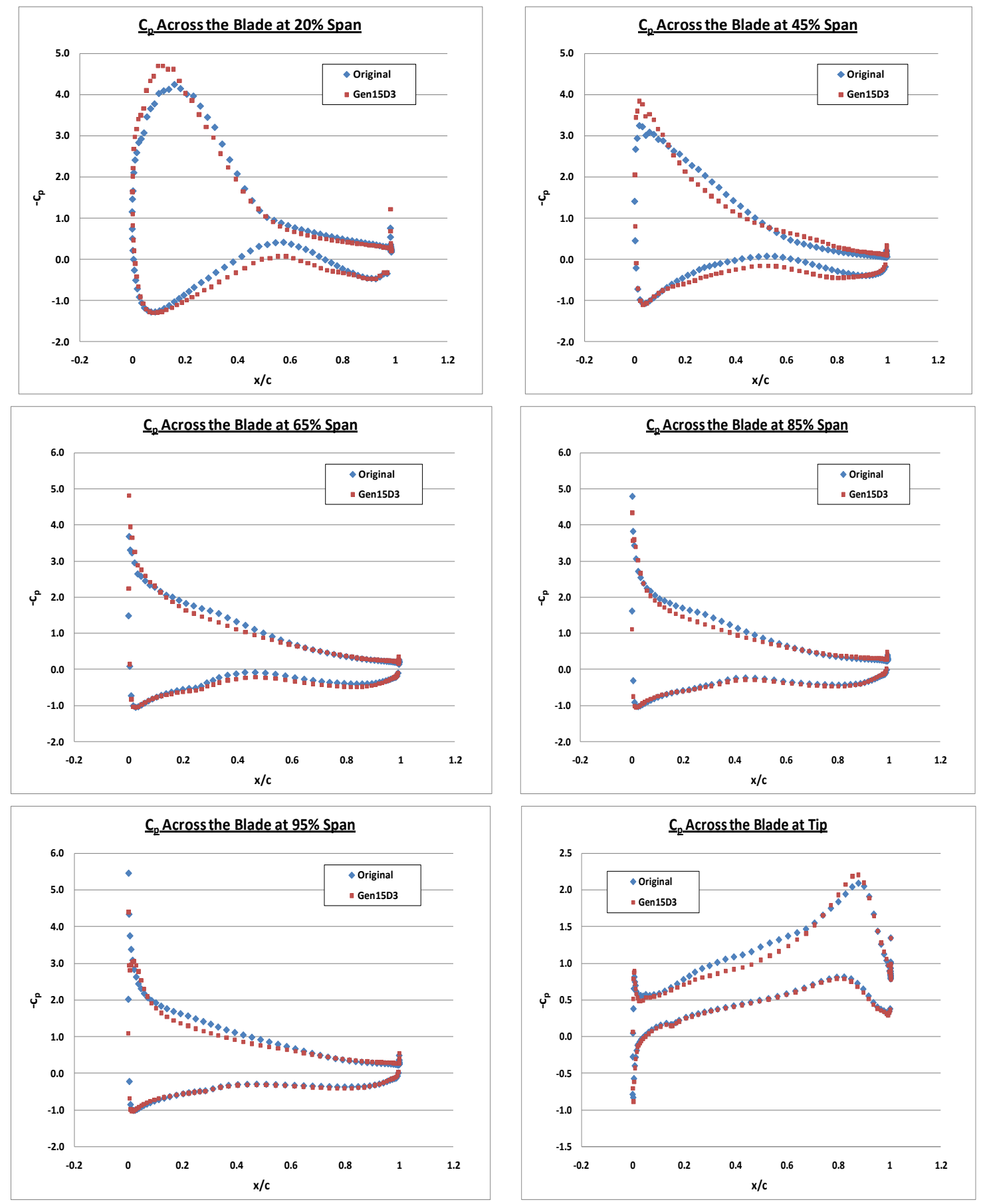

Figure 41: (-Cp) Comparisons of different cross-sections of the original BSDS(blue) wind turbine blade and the G15D3 design (red)

Final Report

42

CRAFTR-08.2011.030

Prime Contract No. DOE-DE-EE0003269

Combustion Research and Flow Technology, Inc. 

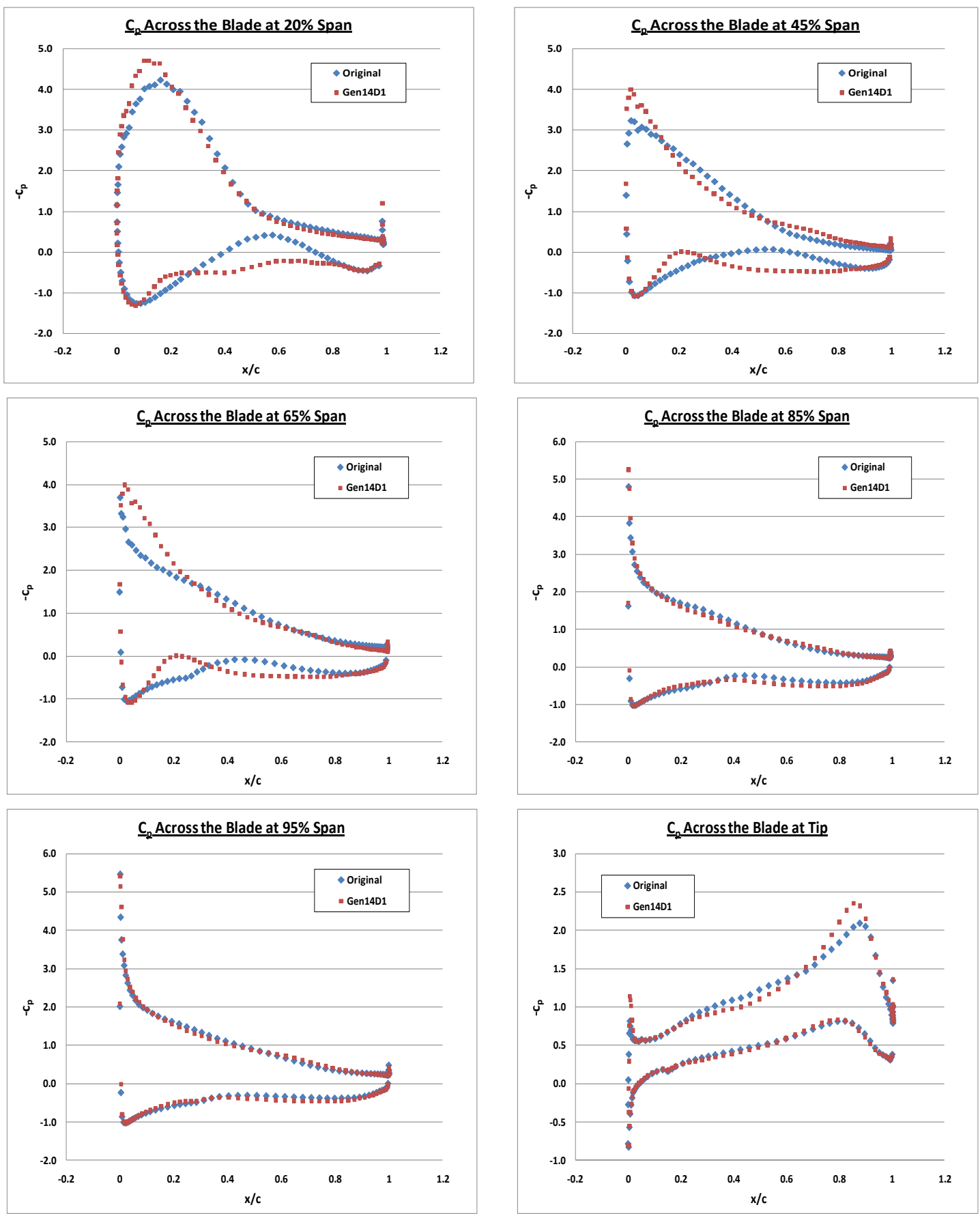

Figure 42: (-Cp) Comparisons of different cross-sections of the original BSDS (blue) wind turbine blade and the G14D1 design(red).

Final Report

Prime Contract No. DOE-DE-EE0003269

Combustion Research and Flow Technology, Inc. 


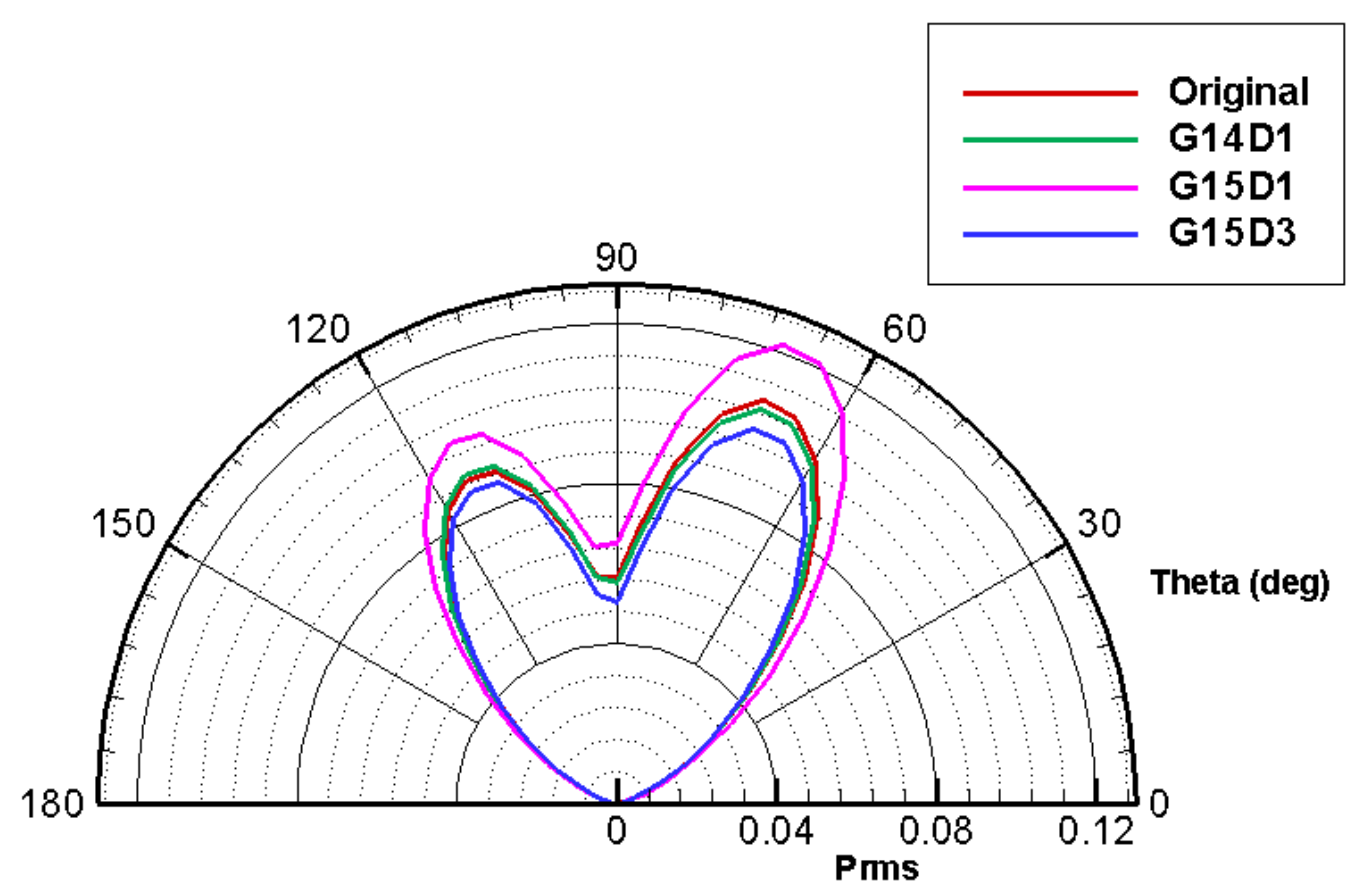

Figure 43: Polar Distribution of RMS Pressure Comparisons for the original BSDS Blade and the three designs.

\subsubsection{Summary of Optimization Studies}

The optimization study for noise reduction and enhancement of power was carried out with the BSDS blade shape, power and noise levels as the baseline reference. As part of the optimization study a Pareto-front that included non-dominated designs in terms of noise and power was identified. Several designs were mined from this front and analyzed for both improvement of aerodynamic power and/or reduction of noise. In particular, three designs were identified: a design that generated approximately the same power as the BSDS design but showed improved tonal noise characteristics; a design that improved both the tonal noise and aerodynamic power marginally and a design that significantly improved aerodynamic power but also increased the tonal noise.

Final Report 


\section{Chapter 4 References}

[1] Barone, M. and Berg, D., "Blade Noise Research at Sandia National Labs", 2010 Blade Workshop, Sandia National Labs, Albuquerque, NM, July, 2010.

[2] Berry, D.S., "Blade System Design Studies Phase II: Final Project Report", Sandia Report SAND2008-4648, July 2008.

[3] Hosangadi, A., Lee, R. A., York, B. J., Sinha, N., and Dash, S. M., "Upwind Unstructured Scheme for Three-Dimensional Combusting Flows," Journal of Propulsion and Power, 12, No. 3, 1996.

[4] Ahuja, V., Shipman, J., Arunajatesan S., and Hosangadi, A., "Multi-Element Unstructured Methodology for Analysis of Turbomachinery Systems" Journal of Propulsion \& Power, Vol. 19, No. 5, pp 945-952, September-October 2003.

[5] Ffowcs Williams, J.E. and Hawkings, D. L., "Sound Generated by Turbulence and Surfaces in Arbitrary Motion", Philosophical Transactions of the Royal Society, A264, 321-342, 1969.

[6] Farassat, F. NASA/TM-2007-214853, "Derivation of Formulations 1 and 1A of Farassat", Langley Research Center, Hampton, VA, 2007.

[7] Ahuja, V., Hosangadi, A., and Lee, Y-T., "Shape Optimization of Multi-Element Airfoils Using Evolutionary Algorithms and Hybrid Unstructured Framework", Paper No. HTFED2004-56374, Proceedings of FEDSM'04: 2004 ASME Heat Transfer/Fluids Engineering Summer Conference, Charlotte, NC, July 11-15, 2004.

[8] Lee, Y.T., Ahuja, V., Hosangadi, A., and Ebert, M., "Shape Optimization of a MultiElement Foil Using an Evolutionary Algorithm", Journal of Fluids Engineering, Vol. 132, No. 5, May 2010.

[9] Lee, Y.T., Ahuja, V., et al. "Impeller Design of a Centrifugal Fan with Blade Optimization", International Journal of Rotating Machinery, Volume 2011, Article ID 537824.

[10] Holland, J.H., Adaptation in Natural and Artificial Systems, MIT Press, 1975.

[11] Goldberg, D.E., Genetic Algorithms in Search, Optimization, and Machine Learning, Addison-Wesley, Reading, MA, 1989.

[12] Carroll, D. L., 1995, "Modeling High Pressure Chemical Oxygen-Iodine Lasers," AIAA Journal, 33, No. 8, pp. 1454-1462.

[13] Fonseca, C. M., and Fleming, P. J., "An Overview of Evolutionary Algorithms in MultiObjective Optimization," Evolutionary Computation, No 3, 1995.

Final Report

Prime Contract No. DOE-DE-EE0003269

Combustion Research and Flow Technology, Inc. 


\section{Appendix D}

Miltec Research \& Technology - Final Report

PAGE D-1

Final Report | DOE Award No. DE-EE0003269 
UNCLASSIFIED

Ducommun Wind Turbine Health Monitoring \& Inflow Predictions

Miltec UM Subcontract \#: 11-10-026

Final Scientific Report
Date prepared: 4 April 2011 Miltec Research and Technology MD911000002-RPT Rev.: New

\section{Wind Turbine Health Monitoring ANd Inflow Predictions}

FINAL SCIENTIFIC REPORT

DOCUMENT \#: MD911000002-RPT

APRIL 8, 2011

CONTRACT NUMBER: DE-EE0003269

SUBCONTRACT NUMBER: 11-10-026

PERIOD: 01 OCTOBER 2010 - 15 APRIL 2011

PREPARED BY:

Miltec Research \& TeChNOLOGY

9 INDUSTRIAL PARK DRIVE

OXFORD, MISSISSIPPI 38655

PHONE: (662) 281-7286

FAX: (662) 281-7261

E-MAIL: JWILLIAMS@ONE.DUCOMMUN.COM

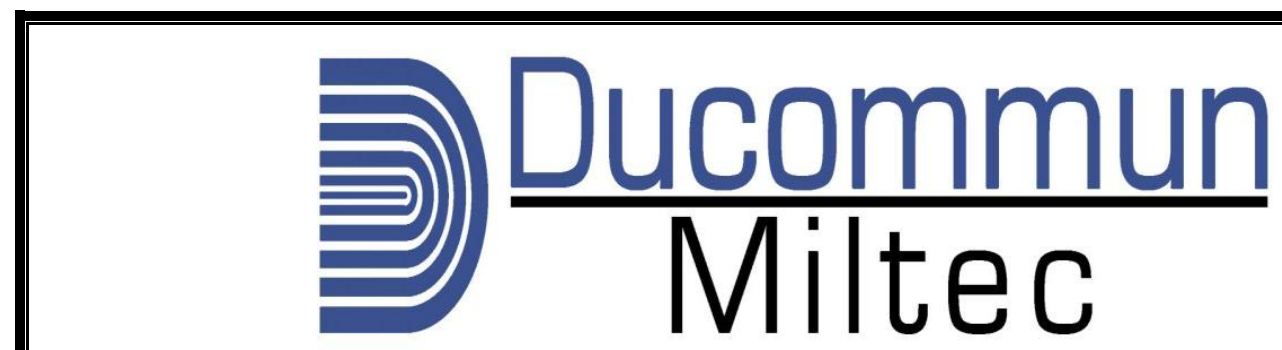

MILTEC PROPRIETARY

Page 1 of 38

UNCLASSIFIED 


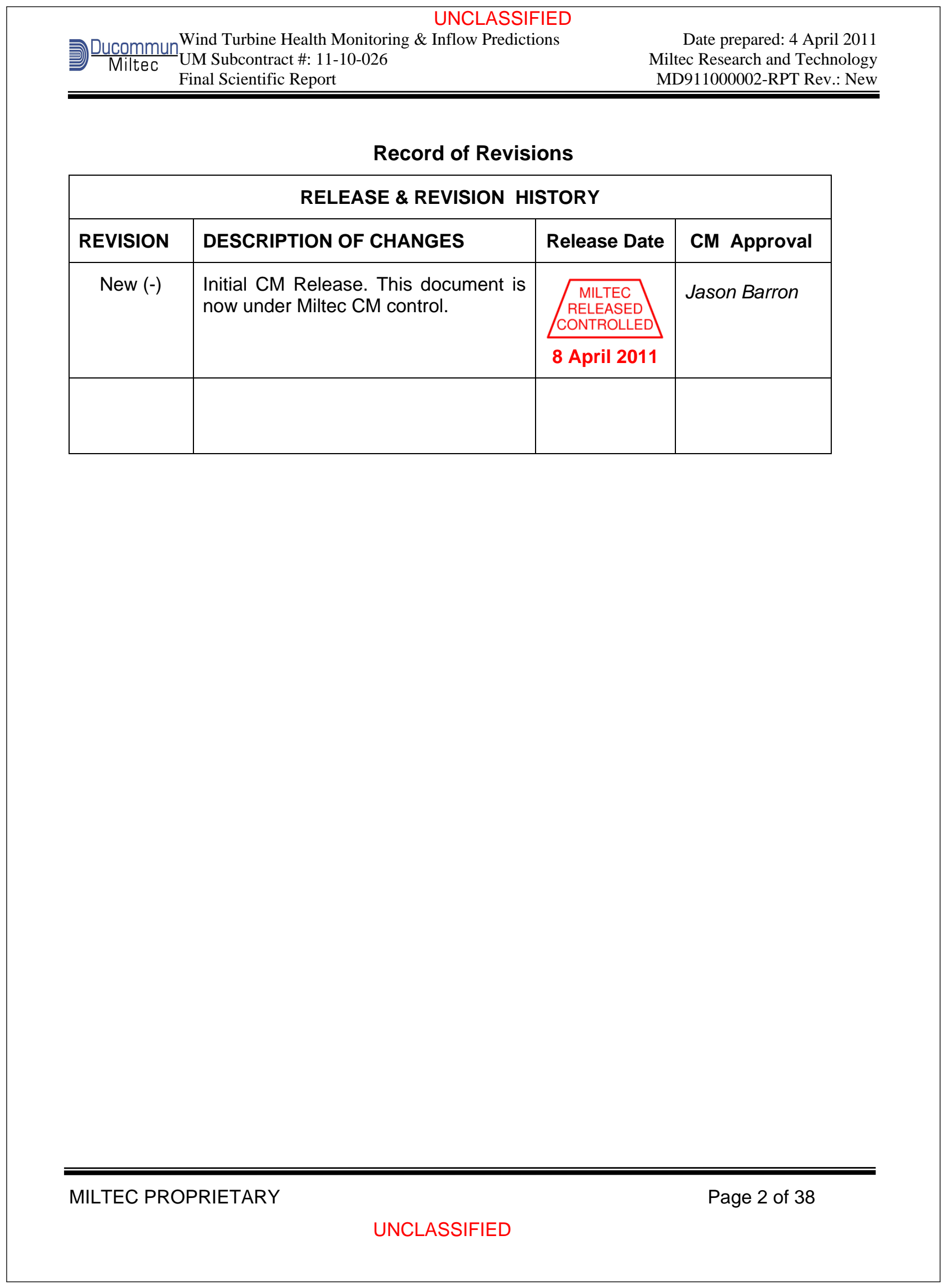

Page D-3

Final Report | DOE Award No. DE-EE0003269 


\section{Table of Contents}

Record of Revisions

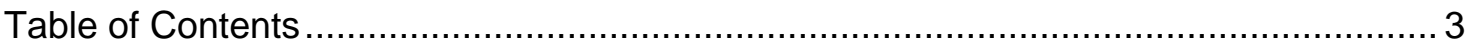

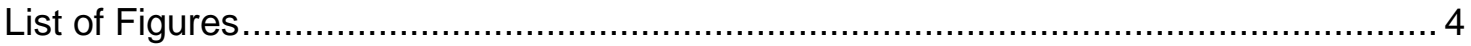

1.0 Task 1 - Health Monitoring of Wind Turbine Blades ...................................... 5

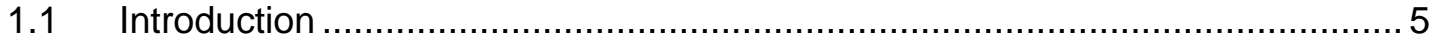

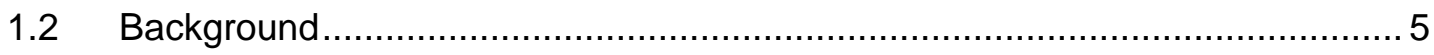

1.3 Wind Turbine Blade Finite Element Models ................................................. 8

1.3.1 Finite Element Model Search............................................................ 8

1.3.2 Generic Blade Finite Element Model .................................................. 10

1.3.3 Identifying Generic Blade Hotspots.................................................. 19

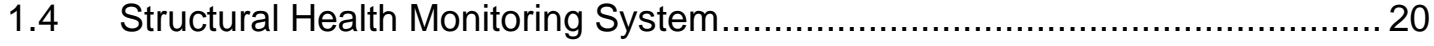

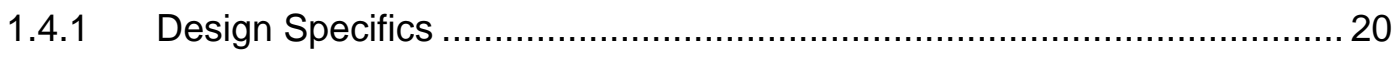

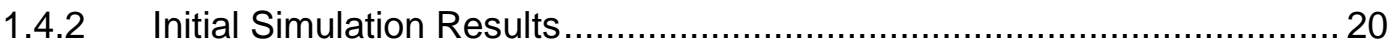

1.4.3 Hypothetical Damage Scenario ...................................................... 24

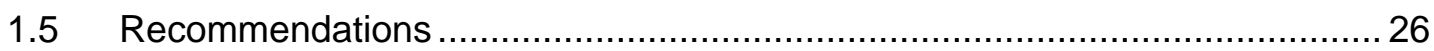

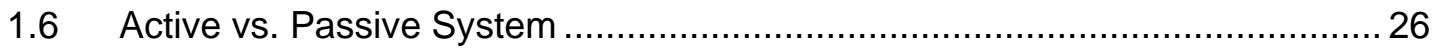

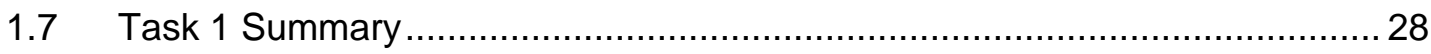

2.0 Task 2 - Passive Detection of Wind Inflow Conditions..................................... 29

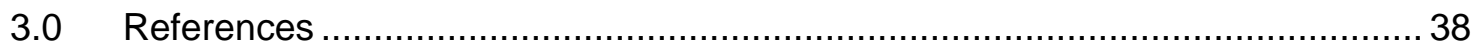




\section{List of Figures}

Figure 1.1 CX-100 blade model.

Figure 1.2 WindPACT blade model. 10

Figure 1.3 Chord length distribution

Figure 1.4 Generic blade section (station 1300-2200) with cross sectional regions labeled.

Figure 1.5 Several views of the generic blade FE mesh.

Figure 1.6 Contour plot of von Mises elastic strain resulting from a static tip load on generic blade; chosen hot spots are outlined in red.

Figure 1.7 Simulation of $10 \%$ Stiffness Reduction at Hotspot 1

Figure 1.8 Simulation of $10 \%$ Stiffness Reduction at Hotspot 2

Figure 1.9 Simulation of $10 \%$ Stiffness Reduction at Hotspot 3 22

Figure 1.10 Simulation of $10 \%$ Stiffness Reduction at Random Location. 22

Figure 1.11 Elements used for simulating crack growth. 24

Figure 1.12 Simulation Results of Crack Growth............................................... 25

Figure 1.13 Health monitoring system design -15 sensors / 1 actuator ..................... 27

Figure 1.14 Health monitoring system design -6 sensors / 1 actuator ....................... 28

Figure 2.1 Modified Google Earth representation of the setup location........................ 30

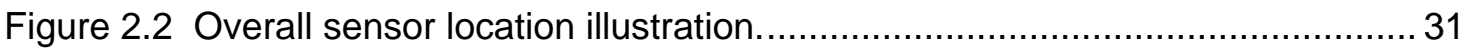

Figure 2.3 Matlab illustration of the distances from center...................................... 31

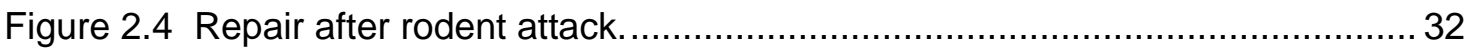

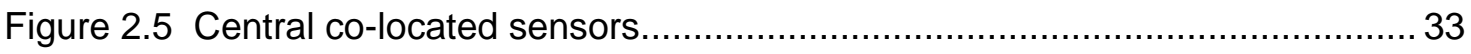

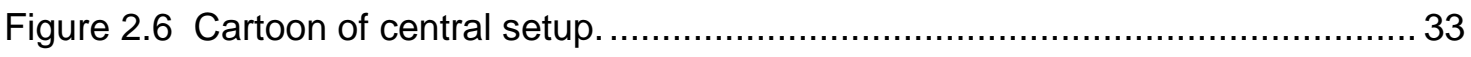

Figure 2.7 Southern-facing view of some of the equipment used for this test. 


\subsection{Task 1 - Health Monitoring of Wind Turbine Blades}

\subsection{Introduction}

Vibration based structural health monitoring (SHM) is a method for detecting damage by monitoring a structure for a change in its dynamic behavior. The dynamic behavior can be characterized using actuators to excite the structure and sensors to measure the structure's response. SHM is an active field of research with most efforts focused on the development of sensors and signal processing techniques. Miltec Research and Technology (MRT) has focused its research efforts in designing where sensors and actuators should be placed on a structure to best detect damage which has traditionally been an area ignored. Designing an optimized health monitoring system has several advantages. The system's sensitivity to detecting damage over the entire structure is maximized while the number of sensors and actuators is minimized. As a result, weight added to the structure is minimized and the system cost and amount of data collected is reduced.

One of MRT's tasks for the National Center for Physical Acoustics (NCPA) is to design a structural health monitoring system for a wind turbine blade, and accomplishing this requires a finite element (FE) model of a blade. Initially our hope was to find a FE model of a non-proprietary blade which could be used to demonstrate MRT's health monitoring design capabilities. MRT pursued several leads in an attempt to acquire a model or at least a complete description of the geometry and material makeup from which a FE model could be created. Unfortunately, our attempts were unsuccessful due to the proprietary nature of many blade designs. As an alternative, MRT used publically available information from several sources to generate a FE model of a generic wind turbine blade. FE models of two wind turbine blades did become available after completing much of this work. However, after some analysis it was determined that the new model would not significantly change the results. Therefore, it was decided to complete the analysis using the MRT developed model.

This report begins with an overview of MRT's health monitoring system design process in Section 1.2. Section 1.3 discusses the wind turbine blade finite element models. The design of a health monitoring system for a generic wind turbine blade is presented in Section 1.4. Multiple optimal designs are generated and their application to a theoretical damage scenario is simulated to judge performance. Two designs are recommended in Section 1.5 and some issues pertaining to the application of an active or passive SHM system to a wind turbine blade is discussed in Section 1.6. Section 1.7 concludes with a summary of the Task 1 effort.

\subsection{Background}

MRT has developed a systematic method for optimally locating sensors and actuators for structural damage detection. The approach is model-based requiring the use of a dynamical model (typically a finite element model) of the structure. When

MILTEC PROPRIETARY

Page 5 of 38

UNCLASSIFIED 
possible, the model is compared against modal analysis test data to ensure it sufficiently represents the structure's dynamics. Modal analysis test data was not available during this effort, but would be a topic to pursue in future work. It is understood in the context of structural modeling that all models have errors and that good designs produced by model-based techniques should be tolerant to these errors. The optimization problem is formulated by specifying the design parameters and constraints, defining the objectives, and selecting a physically measurable damage metric to optimize. The design parameters and constraints specify the type of sensors (acceleration, strain, thermal, etc.) available, sets limits on the number of each sensor type, and defines limits for locating the sensors and actuators. Typical optimization objectives are to 1) be highly sensitive to damage at any known failure location(s), 2) maintain adequate ability to detect damage occurring elsewhere, 3) minimize the number of sensors and actuators used, and 4) maintain robustness to modeling errors. An in-house developed multi-objective genetic algorithm (GA) is employed to perform the optimization. The GA will produce a number of potential health monitoring system designs. Simulations of each design are then performed to validate, judge the performance, and select the most appropriate design.

The framework used by MRT for designing health monitoring systems is founded on the principles of linear, finite-dimensional, time-invariant (LTI) dynamical systems theory. While the linear elastic finite element method (FEM) is appropriate for modeling many structures, MRT's approach is applicable to all systems that can be modeled adequately as a LTI dynamical system in state space form. The state space equations are

$$
\begin{aligned}
& \dot{x}(t)=A x(t)+B u(t) \\
& y(t)=C x(t)+D u(t) \\
& x(0)=x_{0}
\end{aligned}
$$

where $x(t) \in R^{n}$ is the state vector, $u(t) \in R^{m}$ is the actuator signal vector, $y(t) \in R^{p}$ is the sensor signal vector, and $x_{0} \in R^{n}$ is the initial state condition. The matrices $A \in R^{n \times n}$, $B \in R^{n \times m}, C \in R^{p \times n}$, and $D \in R^{p \times p}$ represent the system dynamics, actuator influence, sensor coupling, and direct feed through effects, respectively. Here the symbols $R^{n}$ and $R^{n \times m}$ are used to represent the spaces of $n$-dimensional real vectors and $n$-by- $m$ real matrices, respectively.

The equation of motion resulting from the application of the FEM to a linear elastic structure is,

$$
M \ddot{z}(t)+G \dot{z}(t)+K z(t)=F u(t),
$$

where $M, G$, and $K$, are the mass, damping, and stiffness matrices, respectively. Here, the matrix $F$ maps the actuator signals into the force vector and should be thought of as

MILTEC PROPRIETARY

Page 6 of 38

UNCLASSIFIED 
specifying where independent forces are applied to the structure. The vector $z$ consists of the translational and rotational degrees of freedom (DOF's). Equation 2 is converted to a state space form using the definition

$$
x=\left[\begin{array}{c}
z \\
\dot{z}
\end{array}\right],
$$

and it follows that

$$
A=\left[\begin{array}{cc}
0 & I \\
-M^{-1} K & -M^{-1} G
\end{array}\right] \text { and } B=\left[\begin{array}{c}
0 \\
M^{-1} F
\end{array}\right] .
$$

Any type of sensor may be used provided a mathematical model of the sensor is available. When accelerometers are used for sensing,

$$
y(t)=C_{a} \backslash M^{-1} K \quad-M^{-1} G \underline{\bar{x}}(t)+C_{a} M^{-1} F u(t),
$$

where $C_{a}$ is a matrix specifying the locations of the accelerometers and $F$ is a matrix specifying the locations of the actuators. MRT has typically used piezoelectric disc wafers as actuators. Equation 4 implies that

$$
C=C_{a} \backslash M^{-1} K \quad-M^{-1} G_{-}^{-} \text {and } D=C_{a} M^{-1} F .
$$

The structure of $C_{a}$ is such that each row corresponds to an accelerometer and each column corresponds to a model DOF. A ' 1 ' is placed in the column corresponding to the DOF being measured. The structure of $F$ is such that each column corresponds to an actuator and each row corresponds to a model DOF. A ' 1 ' is placed in the row corresponding to the DOF being excited. Note that no row or column in $C_{a}$ and $F$ can have more than a single ' 1 ' and all other entries should be ' 0 '.

The goal is to optimally position accelerometer(s) and actuator(s) on the structure to detect changes in the structure's dynamics. This corresponds to identifying the best DOF's to measure and excite, i.e., to determine the best $C_{a}$ and $F$ matrices. The structure's steady-state response, defined in (1), to an input (actuator signal) with a covariance matrix $Q_{u}$ is given by

$$
Q_{y}=C Q_{x} C^{T}+D Q_{u} D^{T},
$$

where $Q_{y}$ is the steady-state covariance of the sensor signal $y$ and $Q_{x}$ is the steady-state covariance of the state vector $x$ which can be shown to be the solution to the matrix Lyapunov equation:

$$
A Q_{x}+Q_{x} A^{T}+B Q_{u} B^{T}=0 .
$$

The sensitivity of $Q_{y}$ with respect to parametric variations in the system matrices $A, B$, $C$, and $D$ is given by 


$$
\frac{\partial Q_{y}}{\partial p}=\frac{\partial C}{\partial p} Q_{x} C^{T}+C \frac{\partial Q_{x}}{\partial p} C^{T}+C Q_{x} \frac{\partial C^{T}}{\partial p}+\frac{\partial D}{\partial p} Q_{u} D^{T}+D \frac{\partial Q_{u}}{\partial p} D^{T}+D Q_{u} \frac{\partial D^{T}}{\partial p},
$$

where $\frac{\partial Q_{x}}{\partial p}$ is the solution to

$$
A \frac{\partial Q_{x}}{\partial p}+\frac{\partial Q_{x}}{\partial p} A^{T}+\frac{\partial A}{\partial p} Q_{x}+Q_{x} \frac{\partial A^{T}}{\partial p}+\frac{\partial B}{\partial p} Q_{u} B^{T}+B Q_{u} \frac{\partial B^{T}}{\partial p}=0,
$$

and $p$ is a parameter characterizing the effect of damage on the structure. Damage is modeled as a localized change in the stiffness or mass of a finite element, and this change, represented by the variable $p$, influences the entries of $M, K$, and $G$. Other sensitivity measures are possible, but this one is sensitive to a large space of changes. Note (6) and (8) are of the same form in their unknowns, and readily available numerical algorithms exist to solve these equations.

The 2-norm of (7) is a quantity that can be maximized by MRT's GA to determine optimal sensor and actuator locations. This optimization criterion, $\left\|\partial Q_{y} / \partial p\right\|_{2}$, is a measure of the change in output power covariance in response to a structural parameter change (mass or stiffness reduction). To put this plainly, the algorithm tries to maximize the change in the output signal when damage develops in the structure. In practice this optimization criterion cannot be measured; however, for small changes in $p$, which are of primary interest,

$$
\left\|\frac{\partial Q_{y}}{\partial p}\right\|_{2} \propto\left\|Q_{y}^{r e f}-Q_{y}^{d m g}\right\|
$$

where $Q_{y}^{r e f}$ and $Q_{y}^{d m g}$ are the reference and damaged values of the output power covariance which are quantities that can be measured.

\subsection{Wind Turbine Blade Finite Element Models}

\subsubsection{Finite Element Model Search}

MRT attempted to contact several individuals to request a blade model. MRT has collaborated in the past with Doug Adams at Purdue University who is currently involved in a wind turbine project. Unfortunately the FE model they have developed is proprietary and could not be shared. We have worked previously with Tom Hermann at the National Institute for Aviation Research at Wichita State University. Tom is currently a consultant in the wind turbine field and working for Odonata Research, but did not have any non-proprietary models to share. We also contacted the developers of NuMAD at Sandia National Labs for a blade model, but did not receive a reply. NuMAD is an ANSYS pre-processor designed to aid in creating turbine blade FE models and is available free upon request. 


\section{UNCLASSIFIED}

Ducommun Wind Turbine Health Monitoring \& Inflow Predictions

Miltec UM Subcontract \#: 11-10-026

Final Scientific Report
Date prepared: 4 April 2011 Miltec Research and Technology MD911000002-RPT Rev.: New

Nathan Murray at NCPA was successful in procuring two wind turbine blade FE models. The CX-100 blade is a 9 meter, $100 \mathrm{KW}$ blade designed as a part of a Sandia National Labs research program [1]. The WindPACT blade is a 33 meter, $1.5 \mathrm{MW}$ blade designed as part of a National Renewable Energy Labs program [2]. Figure 1.1 and Figure 1.2 are depictions of each blade model. These models were not used by MRT because they did not become available until very late in the design cycle using a generic blade and after some analysis (presented in Section 1.3.2.5) it was determined that the final results would not be greatly impacted.

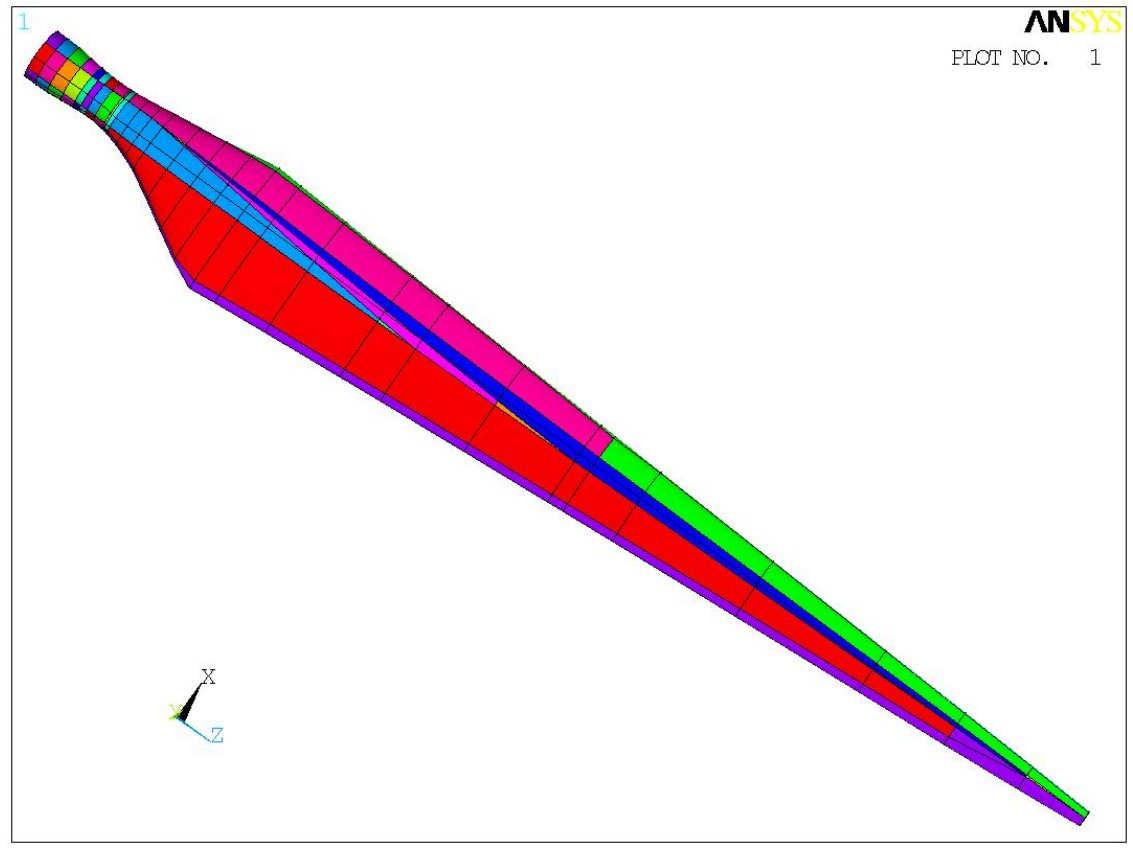

Figure 1.1 CX-100 blade model.

MILTEC PROPRIETARY

Page 9 of 38

UNCLASSIFIED 


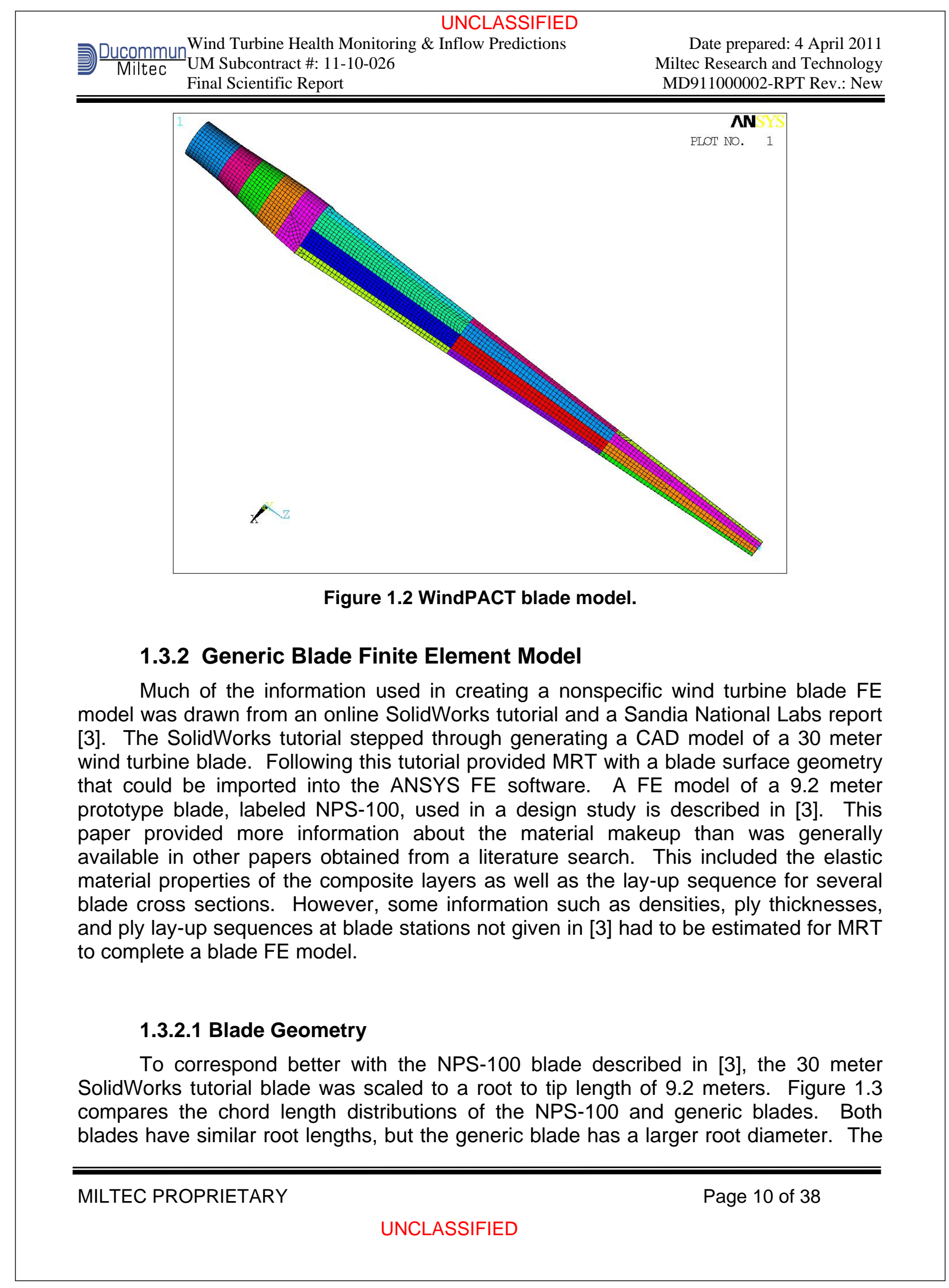




\section{UNCLASSIFIED}

Ducommun Wind Turbine Health Monitoring \& Inflow Predictions

Miltec UM Subcontract \#: 11-10-026

Final Scientific Report
Date prepared: 4 April 2011

Miltec Research and Technology MD911000002-RPT Rev.: New

generic blade has a longer transition region (root to airfoil), but both have similar max chord lengths. Blade thickness versus length for the NPS-100 blade is not provided in [3]. Given that the generic blade has a larger root diameter, it is expected that the generic blade is also thicker than the NPS-100 along the entire blade length. The SolidWorks tutorial does not include any internal structure such as a shear web or spar which are common features in many blade designs. A shear web is used in the NPS100 blade, but its exact location is not given. Based on figures in [3] showing the shear web, a shear web was added to the generic blade model at a similar location. The shear web is normal to the chord length, is offset $50 \mathrm{~mm}$ from the root center towards the leading edge, and extends from blade stations 1300 to $8200 \mathrm{~mm}$.

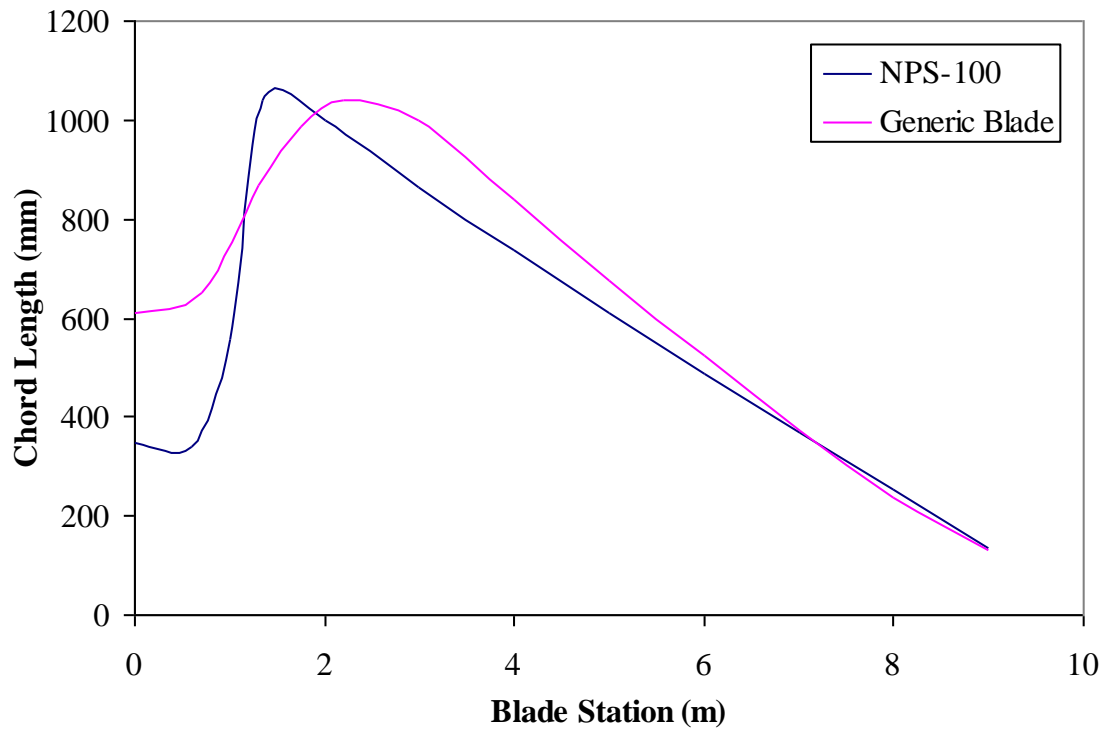

Figure 1.3 Chord length distribution

\subsubsection{Material Properties}

The NPS-100 blade skin and spar cap are composed of e-glass layers with unidirectional (C520), $\pm 45^{\circ}$ (C260), and random (3/4 Mat) fiber orientations. Balsa core material is used in the shear web and trailing edge and the entire blade surface is covered with a gel coat. The properties for each material layer are listed in Table 1.1. The elastic moduli and Poissons' ratio for the e-glass layers and gel coat were provided in [3] along with the thicknesses of the plies composing the DBM layers. Thicknesses for C520, 3/4 Mat, and the gel coat were not explicitly listed but approximated based on a bar graph in [3] plotting total thickness of each material in the spar cap regions. The remaining information in Table 1.1 was not given in [3] and had to be drawn from other

MILTEC PROPRIETARY

Page 11 of 38

UNCLASSIFIED 
sources. The density used for C520 is a typical value for a glass fiber ply according to the Wind Energy Handbook [4]. Because the C260 and 3/4 Mat layers have a lower stiffness than C520, it was assumed they also have a lower fiber volume fraction and were subsequently assigned reduced density values. A web search for gel coats returned a data sheet [5] for an epoxy gel coat manufactured by Gurit from which the gel coat density was obtained. A web search for balsa core materials returned a data sheet [6] for a medium density end grain balsa core manufactured by ProBalsa from which the balsa material properties in Table 1.1 were obtained. A thickness of $6.35 \mathrm{~mm}$ was assumed for balsa used in the skin and $9.5 \mathrm{~mm}$ for balsa used in the shear web.

Table 1.1 Layer material properties

\begin{tabular}{|c|c|c|c|c|c|c|}
\hline Material & $\begin{array}{c}\mathrm{E}_{1} \\
(\mathrm{GPa})\end{array}$ & $\begin{array}{c}\mathrm{E}_{2} \\
(\mathrm{GPa})\end{array}$ & $\begin{array}{c}\mathrm{G}_{12} \\
(\mathrm{GPa})\end{array}$ & $v_{12}$ & $\begin{array}{c}\text { Density } \\
(\mathrm{g} / \mathrm{cc})\end{array}$ & $\begin{array}{c}\text { Thickness } \\
(\mathrm{mm})\end{array}$ \\
\hline $\mathrm{C} 520$ & 48.2 & 11.7 & 6.48 & 0.30 & 1.85 & 1.14 \\
\hline $\mathrm{C} 260$ & 43.0 & 8.90 & 4.50 & 0.27 & 0.925 & 0.186 or 0.296 \\
\hline 3/4 Mat & 7.58 & 7.58 & 6.48 & 0.30 & 0.230 & 0.38 \\
\hline Gel Coat & 3.44 & 3.44 & 1.32 & 0.30 & 1.66 & 0.46 \\
\hline Balsa & 2.00 & 0.40 & 0.025 & 0.20 & 0.155 & 6.35 or 9.5 \\
\hline $\begin{array}{c}\text { DBM1208 } \\
\text { 3 layers }\end{array}$ & $+45^{\circ} \mathrm{C} 260(0.186 \mathrm{~mm}) / 3 / 4 \mathrm{Mat}(0.186 \mathrm{~mm}) /$ & 0.558 \\
\hline DBM1708 & $+45^{\circ} \mathrm{C} 260(0.296 \mathrm{~mm}) / 3 / 4 \mathrm{Mat}(0.296 \mathrm{~mm}) /$ & 0.888 \\
3 layers & \multicolumn{7}{|c|}{$-45^{\circ} \mathrm{C} 260(0.296 \mathrm{~mm})$} \\
\hline
\end{tabular}

\subsubsection{Layered Material Definitions}

The NPS-100 FE model divides the blade cross section into five regions and the blade length into seventeen sections. A layered material definition (ply lay-up sequence) is then defined for each cross sectional/blade station section. This same approach was used in constructing the generic blade FE model. Figure 1.4 shows the 1300-2200 station section of the generic blade and labels the cross sectional regions. The locations of the cross sectional region divisions were estimated based on similar figures given in [3]. The layered material definitions for the cross sectional regions at stations $800,1300,2200$, and 8700 are listed in [3] for the NPS-100 blade. These material definitions were used in the generic blade FE model while the ply lay-up 


\section{UNCLASSIFIED}

Ducommun

Miltec

Wind Turbine Health Monitoring \& Inflow Predictions

UM Subcontract \#: 11-10-026

Final Scientific Report
Date prepared: 4 April 2011 Miltec Research and Technology MD911000002-RPT Rev.: New

sequence for the remaining sections had to be estimated. Layers were either added to or removed from the material definitions given in [3] to create greater skin thickness towards the root and to gradually reduce the skin thickness along the airfoil length (max chord to blade tip). Table 1.2 lists the layered material definitions (LMD) assigned to each cross sectional/blade station section of the generic blade while Table 1.3 gives the ply lay-up sequence for each material definition in Table 1.2.

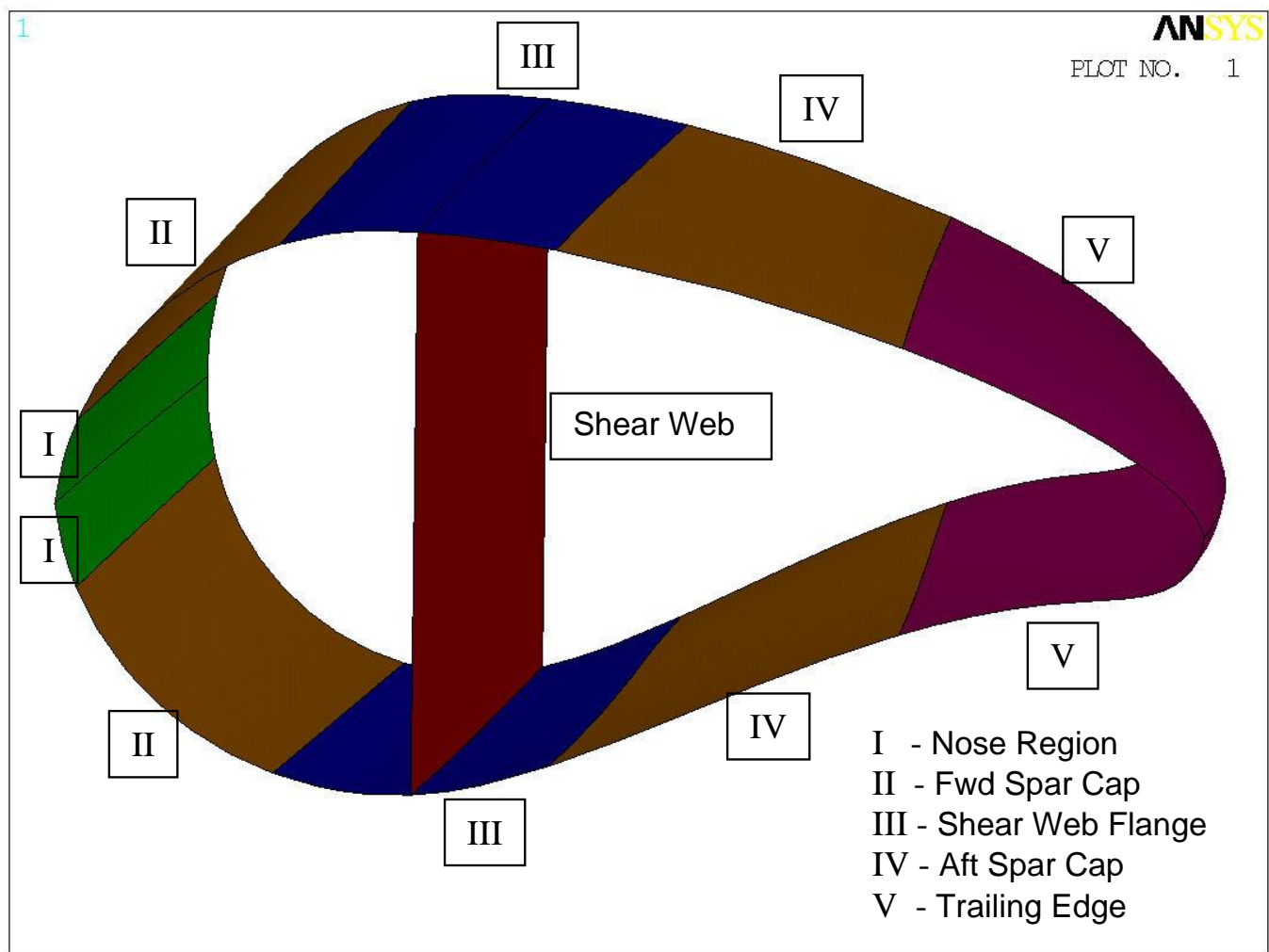

Figure 1.4 Generic blade section (station 1300-2200) with cross sectional regions labeled. 
UNCLASSIFIED

Ducommun Wind Turbine Health Monitoring \& Inflow Predictions

Miltec UM Subcontract \#: 11-10-026

Final Scientific Report
Date prepared: 4 April 2011

Miltec Research and Technology

MD911000002-RPT Rev.: New

Table 1.3 Layered material definitions (type and number of material layers)

\begin{tabular}{|c|c|c|c|c|c|c|c|c|c|}
\hline Material Layer & LMD1 & LMD2 & LMD3 & LMD4 & LMD5* & LMD6* & LMD7* $^{*}$ & LMD8* & LMD9* \\
\hline Gel Coat (exterior) & 1 & 1 & 1 & 1 & 1 & 1 & 1 & 1 & 1 \\
\hline $3 / 4$ Mat & 1 & 1 & 1 & 1 & 1 & 1 & 1 & 1 & 1 \\
\hline DBM1708 & 6 & 4 & 3 & 2 & 1 & 1 & 1 & 1 & 1 \\
\hline DBM1208 & 1 & 1 & 1 & 1 & 1 & 1 & 1 & 1 & 0 \\
\hline C520 & 16 & 14 & 12 & 11 & 10 & 0 & 7 & 7 & 0 \\
\hline Balsa & 0 & 0 & 0 & 0 & 0 & 0 & 0 & 0 & 1 \\
\hline DBM1208 & 1 & 1 & 1 & 1 & 1 & 1 & 1 & 1 & 1 \\
\hline DBM1708 (interior) & 6 & 4 & 3 & 2 & 1 & 1 & 1 & 5 & 0 \\
\hline $\begin{array}{l}\text { Total Thickness } \\
(\mathrm{mm})\end{array}$ & 30.852 & 25.02 & 20.964 & 18.048 & 15.132 & 3.732 & 11.712 & 15.264 & 8.786 \\
\hline
\end{tabular}

* indicates a material definition given in [3] 
UNCLASSIFIED

Ducommun Wind Turbine Health Monitoring \& Inflow Predictions Miltec

M Subcontract \#: 11-10-026 Final Scientific Report
Date prepared: 4 April 2011

Miltec Research and Technology MD911000002-RPT Rev.: New

Table 1.3 cont.

\begin{tabular}{|c|c|c|c|c|c|c|c|c|c|}
\hline Material Layer & LMD11* & LMD12* & LMD13 & LMD14 & LMD15 & LMD16 & LMD17 & LMD18 & LMD1 \\
\hline Gel Coat (exterior) & 1 & 1 & 1 & 1 & 1 & 1 & 1 & 1 & 0 \\
\hline $3 / 4$ Mat & 1 & 1 & 1 & 1 & 1 & 1 & 1 & 1 & 0 \\
\hline DBM1708 & 1 & 1 & 1 & 1 & 1 & 1 & 1 & 1 & 1 \\
\hline DBM1208 & 1 & 1 & 1 & 1 & 1 & 1 & 1 & 0 & 1 \\
\hline C520 & 6 & 6 & 5 & 5 & 4 & 4 & 3 & 0 & 0 \\
\hline Balsa & 0 & 0 & 0 & 0 & 0 & 0 & 0 & 0 & 1 \\
\hline DBM1208 & 1 & 1 & 1 & 1 & 1 & 1 & 1 & 1 & 1 \\
\hline DBM1708 (interior) & 1 & 5 & 1 & 4 & 1 & 3 & 1 & 0 & 1 \\
\hline $\begin{array}{l}\text { Total Thickness } \\
(\mathrm{mm})\end{array}$ & 10.572 & 14.124 & 9.432 & 12.096 & 8.292 & 10.068 & 7.152 & 2.286 & 12.39 \\
\hline
\end{tabular}

MILTEC PROPRIETARY

Page 16 of 38

UNCLASSIFIED 


\subsubsection{Meshed Generic Blade}

Meshing the blade geometry, along with the material properties and layered material definitions, completes the generic blade FE model. Figure 1.5 shows the FE mesh consisting of 8-noded shell elements. In all there are 1,266 elements and 3,735 nodes with each node having 6 degrees of freedom ( 3 translational and 3 rotational). All degrees of freedom at the root end were constrained, positioning the blade in a cantilevered position, and giving a total of 22,194 model degrees of freedom.

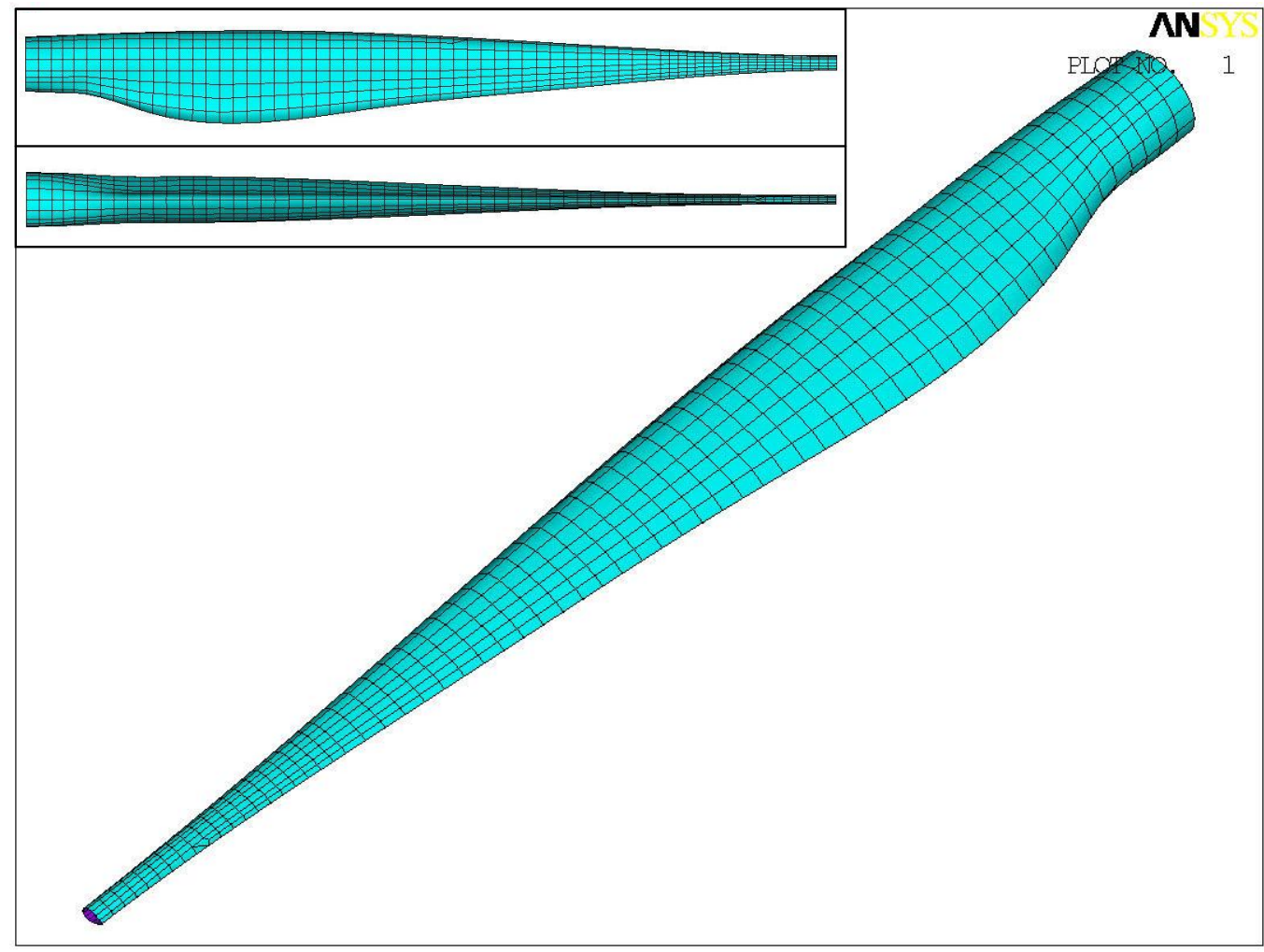

Figure 1.5 Several views of the generic blade FE mesh.

\subsubsection{Comparison to NPS-100 and CX-100 Blades}

Provided in [3] are several FE analysis results used to check the validity of the NPS-100 FE model. These include a calculation of the blade's total mass, a static analysis to determine tip deflection under load, and a modal analysis to compute the first six natural frequencies. The static analysis loading condition consisted of four concentrated $250 \mathrm{lb}$ loads at blade stations 3.0, 4.5, 6.5, and 8.0 meters. The modal analysis was conducted with the root end fixed. Identical simulations were conducted

MILTEC PROPRIETARY

Page 17 of 38

UNCLASSIFIED 

MD911000002-RPT Rev.: New

on the CX-100 and generic blade FE models. This allowed for a comparison of the generic blade to the NPS-100 and CX-100 prototype blades giving an indication of how close the generic blade's mass, flapwise stiffness, and dynamic behavior are to an actual turbine blade.

Table 1.4 summarizes the FE analysis results for the NPS-100 and CX-100 blades and for an initial and a modified generic blade FE model. The initial model uses the material property values listed in Table 1.1 and the modified model has these material property values altered. Given that the generic blade has a larger root diameter and blade thickness than the NPS-100, the generic blade's mass being $27 \mathrm{~kg}$ $(60 \mathrm{lb})$ heavier is not unreasonable. The CX-100 blade has carbon fiber incorporated in its design resulting in a reduced mass compared to the all fiber glass NPS-100 and generic blades. However, the tip deflection and natural frequencies for the initial generic blade model indicate that the blade could be overly stiff. The blade's stiffness could be reduced by adjusting the layer thicknesses, the number or type of layers used in a ply lay-up sequence, or the material properties. In this case, the simplest model parameter to adjust is the material properties. The elastic moduli of the e-glass layers (C520, C260, and 3/4 Mat) were reduced by 75\% from the values in Table 1.1 bringing the tip deflection and natural frequencies closer to those of the NPS-100 blade. Despite the generic blade being a conglomeration, the results in Table 1.4 indicate its dynamic behavior should be similar to that of an actual wind turbine blade.

Table 1.4 Comparison of NPS-100 and CX-100 to generic blade FE analysis results

\begin{tabular}{|c|c|c|c|c|}
\hline & \multirow{2}{*}{$\begin{array}{c}\text { NPS-100 } \\
\text { Blade }\end{array}$} & $\begin{array}{c}\text { CX-100 } \\
\text { Blade }\end{array}$ & \multicolumn{2}{|c|}{ Generic Blade } \\
\cline { 4 - 5 } & $195 \mathrm{~kg}$ & $174 \mathrm{~kg}$ & $222 \mathrm{~kg}$ & $222 \mathrm{~kg}$ \\
\hline Blade Mass & $190 \mathrm{~mm}$ & $80 \mathrm{~mm}$ & $30 \mathrm{~mm}$ & $117 \mathrm{~mm}$ \\
\hline Tip Deflection & $4.52 \mathrm{~Hz}$ & $4.17 \mathrm{~Hz}$ & $10.96 \mathrm{~Hz}$ & $5.56(\mathrm{~Hz})$ \\
\hline $1^{\text {st }}$ Mode (flapwise) & $7.13 \mathrm{~Hz}$ & $5.20 \mathrm{~Hz}$ & $13.23 \mathrm{~Hz}$ & $6.84(\mathrm{~Hz})$ \\
\hline $2^{\text {nd }}$ Mode (edgewise) & $12.27 \mathrm{~Hz}$ & $11.39 \mathrm{~Hz}$ & $25.45 \mathrm{~Hz}$ & $13.02(\mathrm{~Hz})$ \\
\hline $3^{\text {rd }}$ Mode (mixed) & $22.43 \mathrm{~Hz}$ & $17.47 \mathrm{~Hz}$ & $35.49 \mathrm{~Hz}$ & $18.39(\mathrm{~Hz})$ \\
\hline $4^{\text {th }}$ Mode (mixed) & $24.58 \mathrm{~Hz}$ & $21.55 \mathrm{~Hz}$ & $43.60 \mathrm{~Hz}$ & $22.90(\mathrm{~Hz})$ \\
\hline $5^{\text {th }}$ Mode (mixed) & $24.82 \mathrm{~Hz}$ & $31.11 \mathrm{~Hz}$ & $53.90 \mathrm{~Hz}$ & $28.83(\mathrm{~Hz})$ \\
\hline $6^{\text {th }}$ Mode (mixed) & $34.90 d i f i e d$ Model \\
\hline
\end{tabular}


UNCLASSIFIED

Wind Turbine Health Monitoring \& Inflow Predictions

Miltec UM Subcontract \#: 11-10-026

Final Scientific Report
Date prepared: 4 April 2011

Miltec Research and Technology MD911000002-RPT Rev.: New

\subsubsection{Identifying Generic Blade Hotspots}

A static analysis was performed by applying a load at the blade tip with the root end fixed to create bending in the blade and look for resulting areas with high stress or strain. This was done to determine regions, or hotspots, of the blade that may be more susceptible to damage. The health monitoring system can then be designed to be more sensitive to damage occurring in these hotspots. Figure 1.6 is a contour plot of von Mises strain with the selected hotspots outlined in red. There were also high strain regions towards the trailing edge, but these are an artifact of how the FE model is constructed. These areas are along sectional boundaries of the blade model where the skin thickness changes sharply due to different layered material definitions. The first hot spot is where the shear web terminates, the second is along the shear web/skin interface, and the third is in the blade root to airfoil transition region. Two papers describing a static load test [7] and a fatigue test [8] on a 9 meter blade showed cracking in locations similar to hotspots 1 and 2, further suggesting that these areas are likely locations for damage.

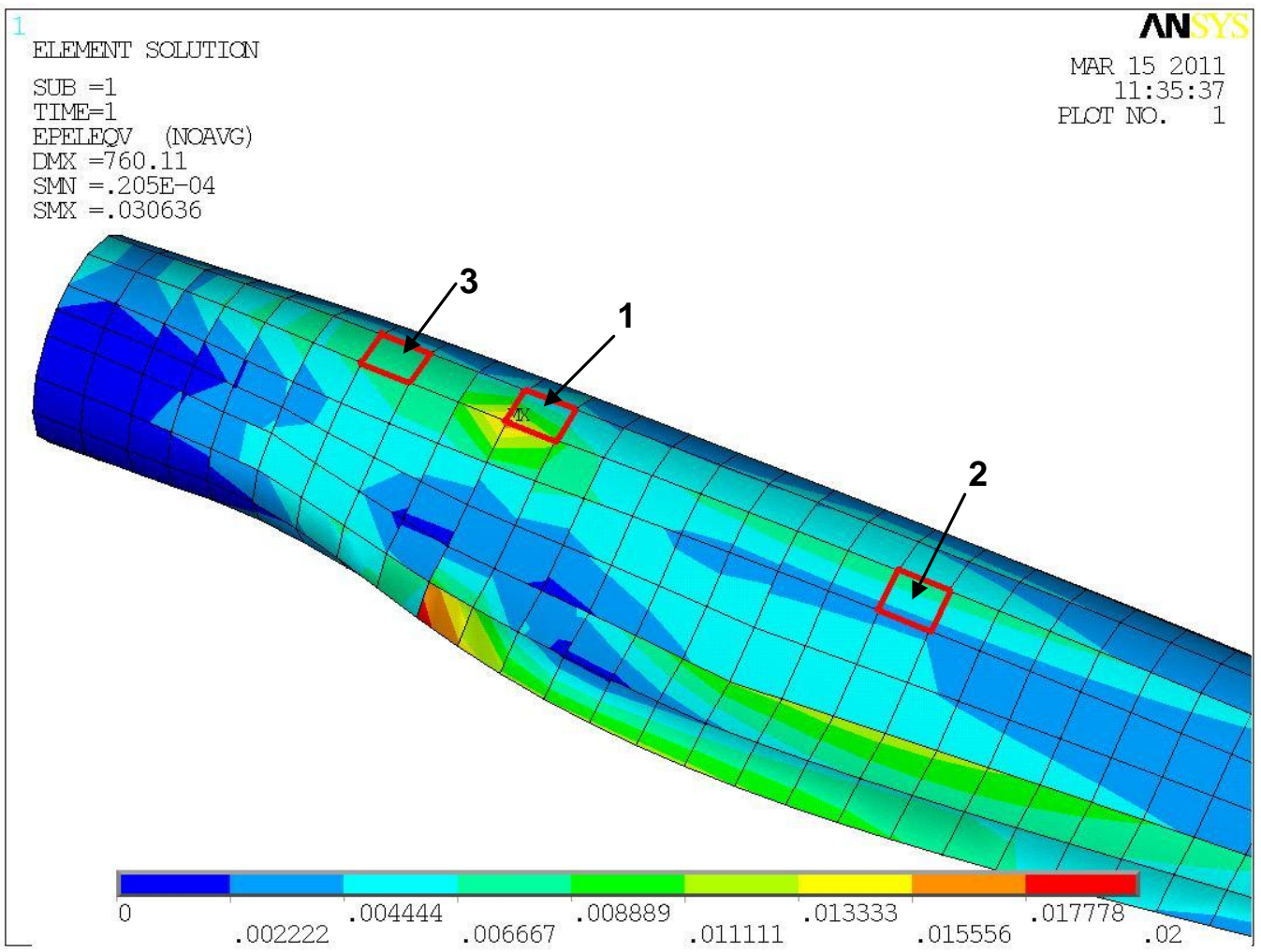

Figure 1.6 Contour plot of von Mises elastic strain resulting from a static tip load on generic blade; chosen hot spots are outlined in red.

MILTEC PROPRIETARY

Page 19 of 38

UNCLASSIFIED 


\subsection{Structural Health Monitoring System}

\subsubsection{Design Specifics}

After the generic FE model was finalized in ANSYS, the mass and stiffness matrices were extracted and reduced to only include modes up to $1,000 \mathrm{~Hz}$. This resulted in a modal reduction from 22,194 DOF to 428 which allows for calculations to be preformed efficiently. Objective functions were then developed in order to specify "what is optimal" to this particular task. Seven objective functions were defined as follows:

- Maximize sensitivity to a reduction in stiffness occurring at the three hot spots mentioned above

- Remain sensitive to randomly occurring damage

- Limit the number of sensors to be between 5-16

- Limit the number of actuators to 1

- Robustness to modeling error

The allowable locations for the sensors and actuator to be placed were limited to the blade surface between stations 500 and 8500 (excluding the leading and trailing edges). This was chosen to make follow on experiments easily implementable. For an actual design intended for use the inside surface of the blade would also be considered. Using MRT's genetic algorithm and the objective functions and constraints listed above, twelve optimal SHM designs were found. Next, these designs were simulated based on various damage cases to gauge the performance and to select the most appropriate design(s).

\subsubsection{Initial Simulation Results}

Four damage locations were selected for simulation purposes; the three hot spots, specified in the objective functions and shown in Figure 1.6, as well as one random location. To simulate cracking, mass and stiffness matrices were extracted from ANSYS with a 10\% reduction in stiffness in each of those elements independently. The stiffness was reduced by decreasing the elastic modulus of the C520 material along the material's strong direction $\left(E_{1}\right)$. The simulated responses of the monitoring system are shown in Figures 1.7-1.10. In these figures each data point represents a run of the SHM system. Data points 1-10 are a baseline (runs on an undamaged blade) with damage being introduced at data point 11. In an actual application a baseline data set is collected so that additional data can be compared back to the baseline to statistically determine if damage has occurred. 


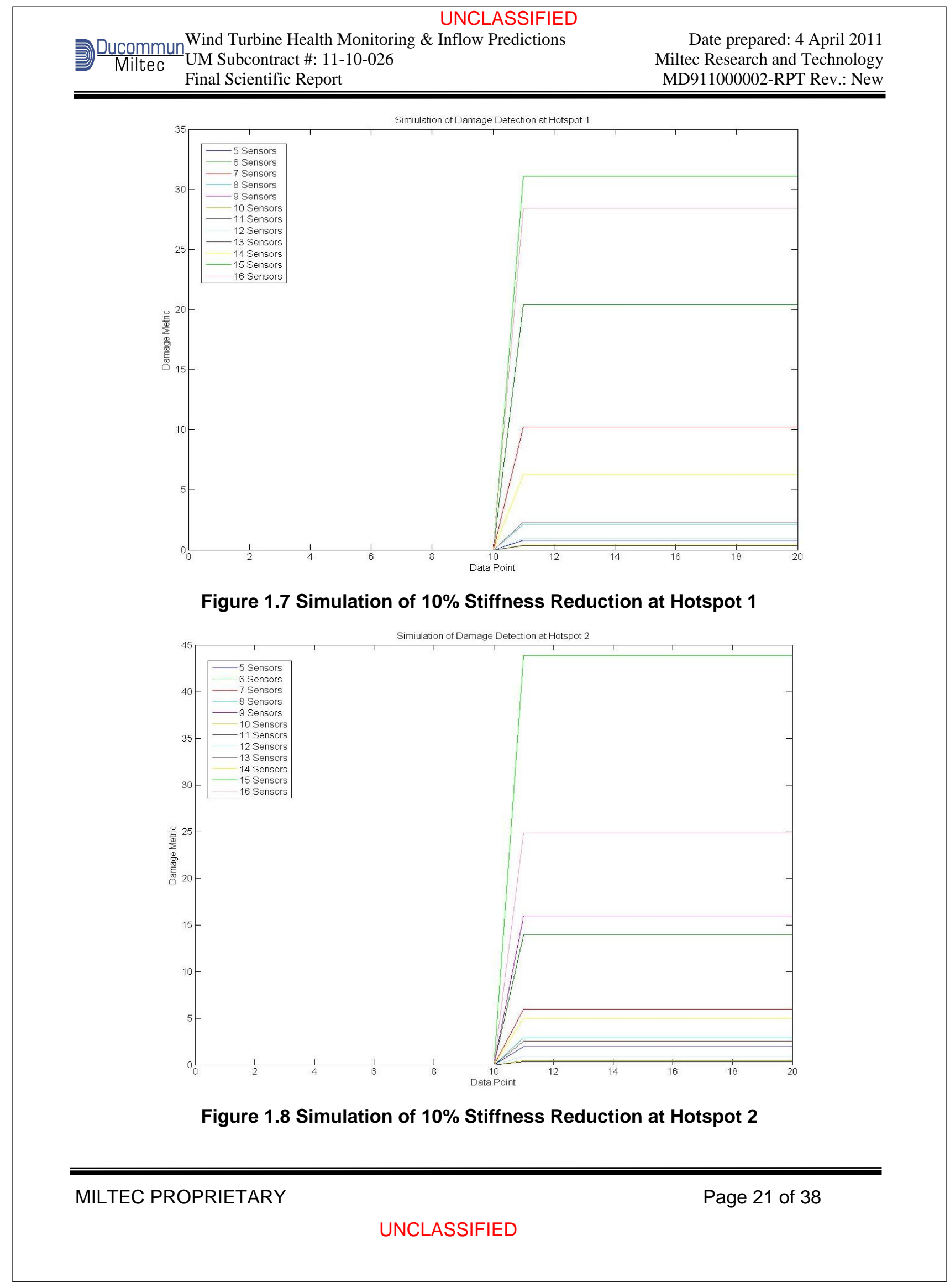

PAGe D-22

Final Report | DOE Award No. DE-EE0003269 


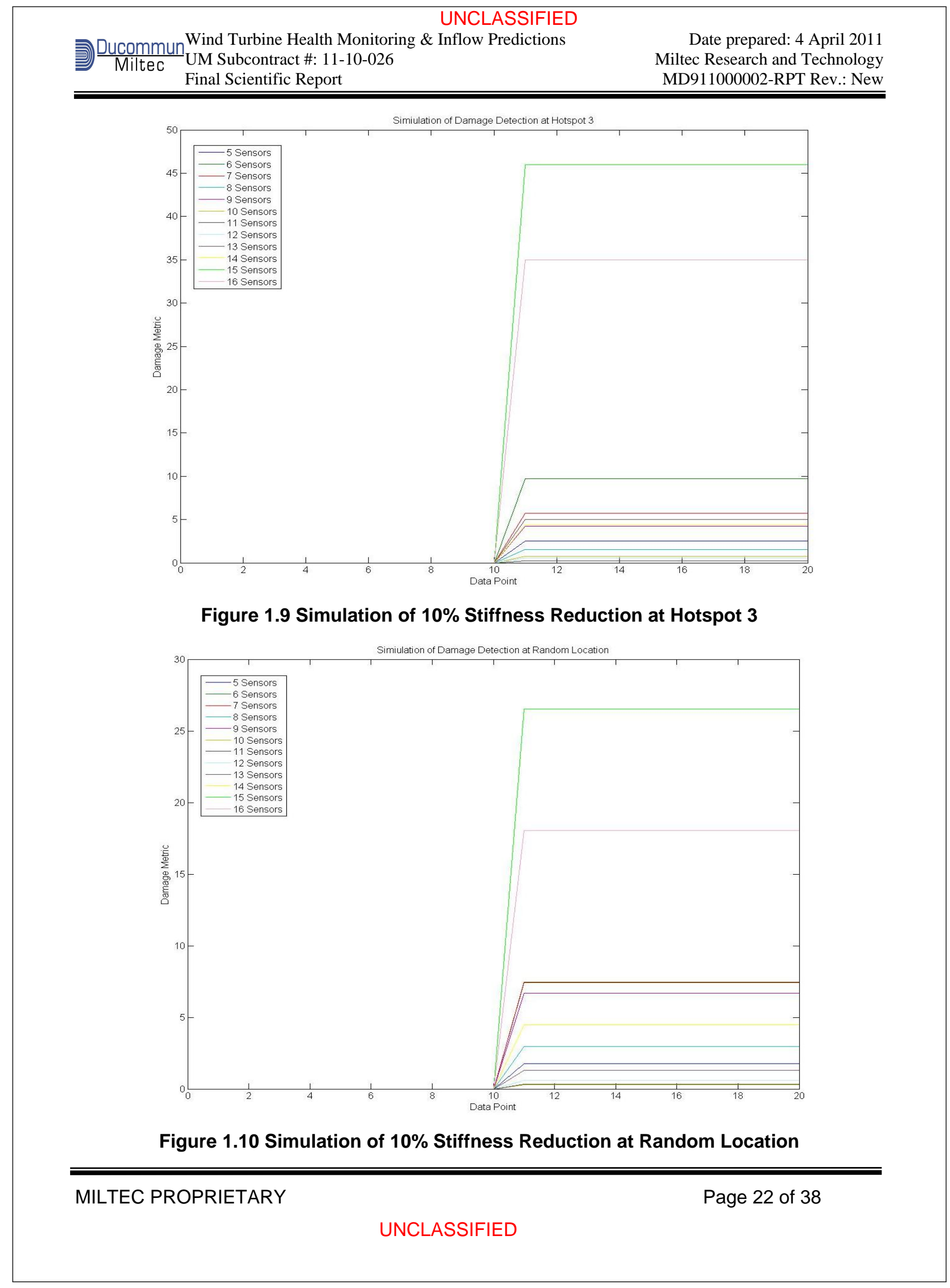

Page D-23

Final Report | DOE Award No. DE-EE0003269 
UNCLASSIFIED

Ducommun Wind Turbine Health Monitoring \& Inflow Predictions

Miltec UM Subcontract \#: 11-10-026

Final Scientific Report
Date prepared: 4 April 2011 Miltec Research and Technology MD911000002-RPT Rev.: New

As one would expect, an increase in the number of sensors typically results in an increase in metric performance. However, since all designs are optimized, they are all equally valid. This allows for the engineer to apply judgment (cost, weight, DAQ, etc.) when selecting which optimal design to use and to know how the number of sensors affects performance. For example, if the engineer is limited to eight inputs on the DAQ equipment, then a design can be chosen based on that constraint. If cost is the most constraining factor, a design can be chosen based on the number of sensors the engineer can purchase.

Table 1.5 contains the output of MRT's genetic algorithm showing the performance trade-off for each optimal design based on number of sensors. These values are each design's sensitivity to a change in stiffness at the three hotspots as well as all other locations (observability). This will show the engineer the sensitivity or performance loss when, for example, a five sensor design is chosen over a ten.

Table 1.5 Performance Trade-off of Optimal Designs

\begin{tabular}{|c|c|c|c|c|}
\hline $\begin{array}{c}\text { \# of } \\
\text { Sensors }\end{array}$ & $\begin{array}{c}\text { Hotspot 1 } \\
\text { Sensitivity } \\
(\mathbf{V} / \mathbf{V})\end{array}$ & $\begin{array}{c}\text { Hotspot 2 } \\
\text { Sensitivity } \\
(\mathbf{V} / \mathrm{V})\end{array}$ & $\begin{array}{c}\text { Hotspot 3 } \\
\text { Sensitivity } \\
(\mathbf{V} / \mathrm{V})\end{array}$ & $\begin{array}{c}\text { Observability } \\
\text { (V/V) }\end{array}$ \\
\hline $\mathbf{5}$ & $1.04 \mathrm{E}-07$ & $7.34 \mathrm{E}-08$ & $1.04 \mathrm{E}-07$ & $1.51 \mathrm{E}-15$ \\
\hline $\mathbf{6}$ & $1.29 \mathrm{E}-08$ & $5.90 \mathrm{E}-07$ & $1.29 \mathrm{E}-08$ & $3.85 \mathrm{E}-15$ \\
\hline $\mathbf{7}$ & $2.67 \mathrm{E}-08$ & $3.94 \mathrm{E}-07$ & $2.67 \mathrm{E}-08$ & $1.32 \mathrm{E}-14$ \\
\hline $\mathbf{8}$ & $1.42 \mathrm{E}-08$ & $2.01 \mathrm{E}-07$ & $1.42 \mathrm{E}-08$ & $9.27 \mathrm{E}-13$ \\
\hline $\mathbf{9}$ & $4.56 \mathrm{E}-08$ & $1.84 \mathrm{E}-07$ & $4.56 \mathrm{E}-08$ & $5.97 \mathrm{E}-11$ \\
\hline $\mathbf{1 0}$ & $6.81 \mathrm{E}-08$ & $5.24 \mathrm{E}-09$ & $6.81 \mathrm{E}-08$ & $1.53 \mathrm{E}-09$ \\
\hline \hline $\mathbf{1 1}$ & $1.86 \mathrm{E}-08$ & $4.42 \mathrm{E}-08$ & $1.86 \mathrm{E}-08$ & $9.51 \mathrm{E}-09$ \\
\hline $\mathbf{1 2}$ & $1.32 \mathrm{E}-08$ & $7.84 \mathrm{E}-08$ & $1.32 \mathrm{E}-08$ & $6.90 \mathrm{E}-08$ \\
\hline $\mathbf{1 3}$ & $1.29 \mathrm{E}-08$ & $3.34 \mathrm{E}-08$ & $1.29 \mathrm{E}-08$ & $1.63 \mathrm{E}-07$ \\
\hline \hline $\mathbf{1 4}$ & $2.01 \mathrm{E}-07$ & $7.34 \mathrm{E}-08$ & $2.01 \mathrm{E}-07$ & $4.50 \mathrm{E}-07$ \\
\hline $\mathbf{1 5}$ & $2.12 \mathrm{E}-07$ & $7.45 \mathrm{E}-07$ & $2.12 \mathrm{E}-07$ & $1.43 \mathrm{E}-06$ \\
\hline $\mathbf{1 6}$ & $1.69 \mathrm{E}-06$ & $7.53 \mathrm{E}-06$ & $1.69 \mathrm{E}-06$ & $3.88 \mathrm{E}-06$ \\
\hline
\end{tabular}




\section{UNCLASSIFIED}

Ducommun Wind Turbine Health Monitoring \& Inflow Predictions

Miltec UM Subcontract \#: 11-10-026

Final Scientific Report
Date prepared: 4 April 2011 Miltec Research and Technology MD911000002-RPT Rev.: New

\subsubsection{Hypothetical Damage Scenario}

The SHM designs were applied to a hypothetical damage scenario to illustrate and compare their performance. Damage is simulated by reducing the stiffness of an element or group of elements. In this case a crack growing through four elements is simulated by successively reducing the stiffness in each element. The stiffness was reduced by decreasing the $E_{1}$ modulus of the C520 material. Figure 1.11 shows the location of the four elements through which the crack grows and the percent reduction in stiffness for each element is given in Table 1.6. Figure 1.12 shows the simulation results for each design and each damage case. The 15 sensor design performs the best for these particular damage conditions, but since all designs are optimal, the engineer can select which design to use based on more than just simulated performance at one or two locations.

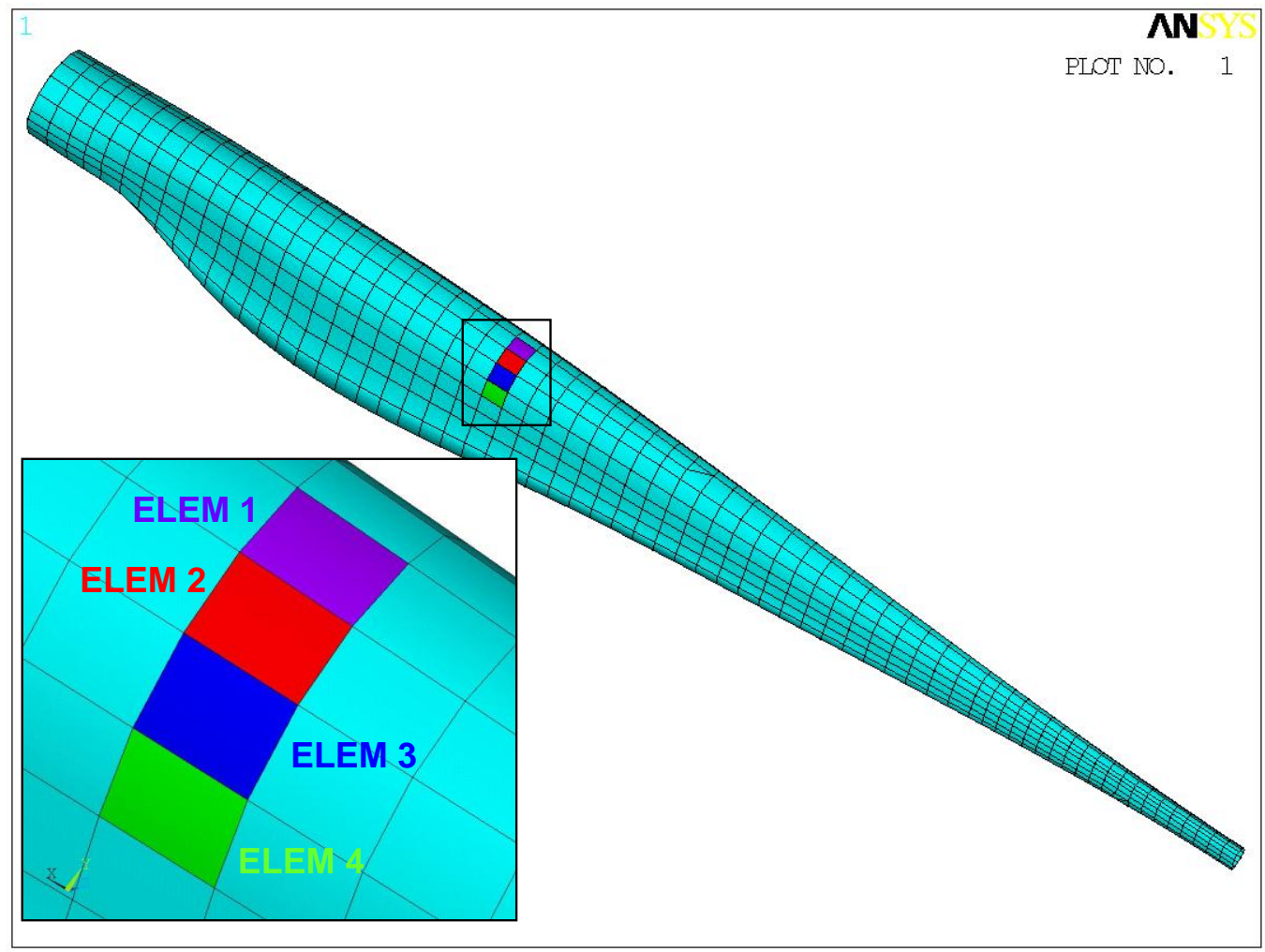

Figure 1.11 Elements used for simulating crack growth.

MILTEC PROPRIETARY

Page 24 of 38

UNCLASSIFIED 


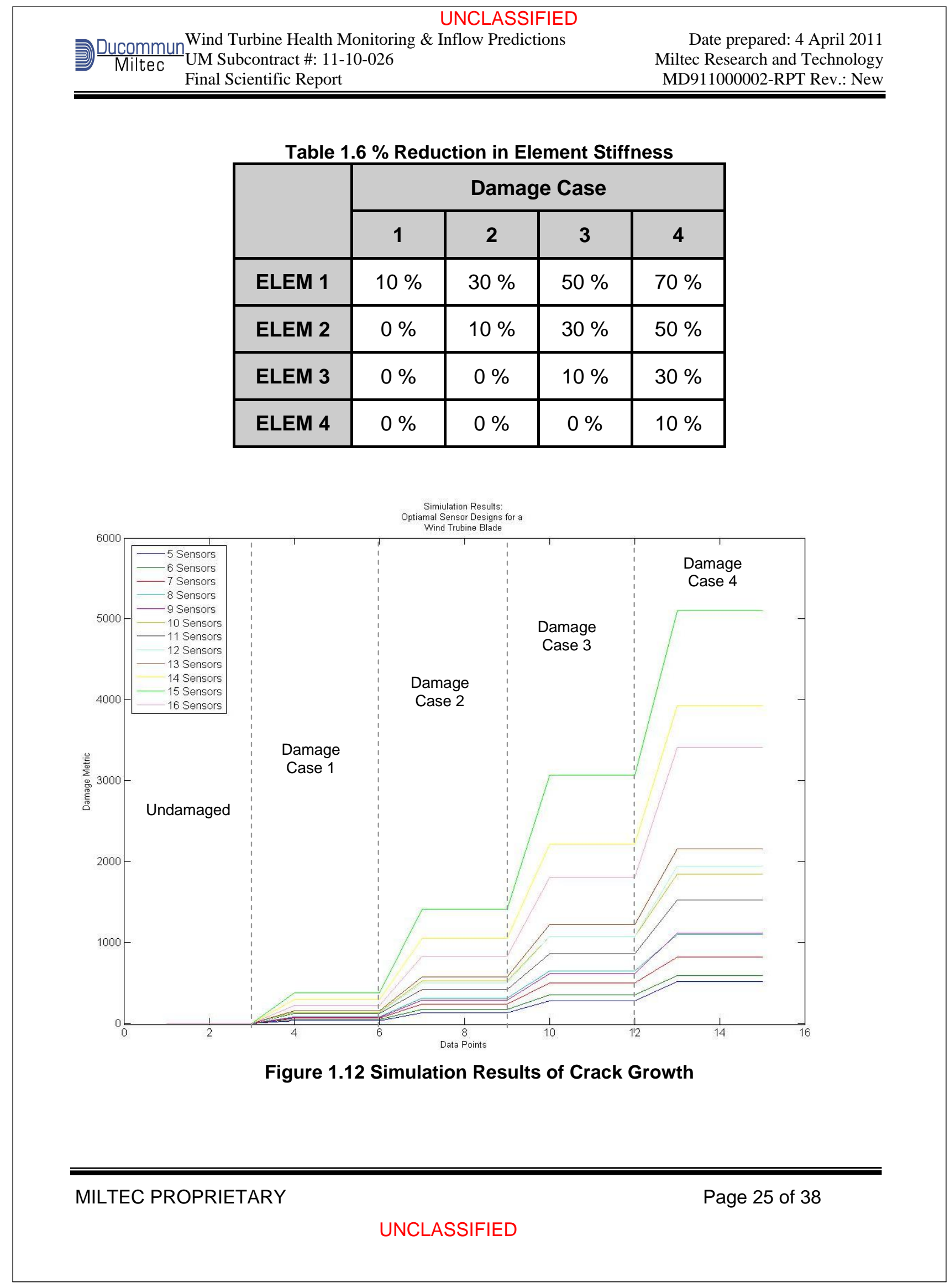

Page D-26

Final Report | DOE Award No. DE-EE0003269 


\subsection{Recommendations}

Based on the simulation results presented in Sections 1.4.2 and 1.4.3, all the designs would have the necessary sensitivity to detect moderate levels of damage. However, the 15 sensor design would be recommended to achieve maximum sensitivity to stiffness changes. If the number of sensors needs to be minimized, based on constraints such as cost, DAQ, weight, etc., while still achieving needed performance, the 6 sensor design would then be recommended. The 15 sensor and 6 sensor designs are depicted in Figure 1.13 and Figure 1.14, respectively.

\subsection{Active vs. Passive System}

These SHM designs are all active systems that operate by exciting the blade via the actuator. The excitation signal used in these simulations is a sine sweep ranging from 500 to $1000 \mathrm{~Hz}$ to excite all modes in this frequency band. If an active system is unacceptable a passive system can be designed where the environmental loads on a blade are utilized as the excitation source. In this case, the spectrum of the wind turbulence is sufficient to excite the structure; however, the noise floor of the baseline data would be higher than for an active system. The result would be that a passive system will not be able to reliably detect small amounts of damage but will perform adequately once the damage grows. As an example, a passive system may have a damage metric noise floor of 500. In this scenario the first damage case in Figure 12 would be undetectable and the 5, 6, and 7 sensor designs would not be viable options. The remaining designs would be effective if implemented as a passive system although their performance would be somewhat reduced. Despite these drawbacks we are confident a passive SHM system is feasible. 


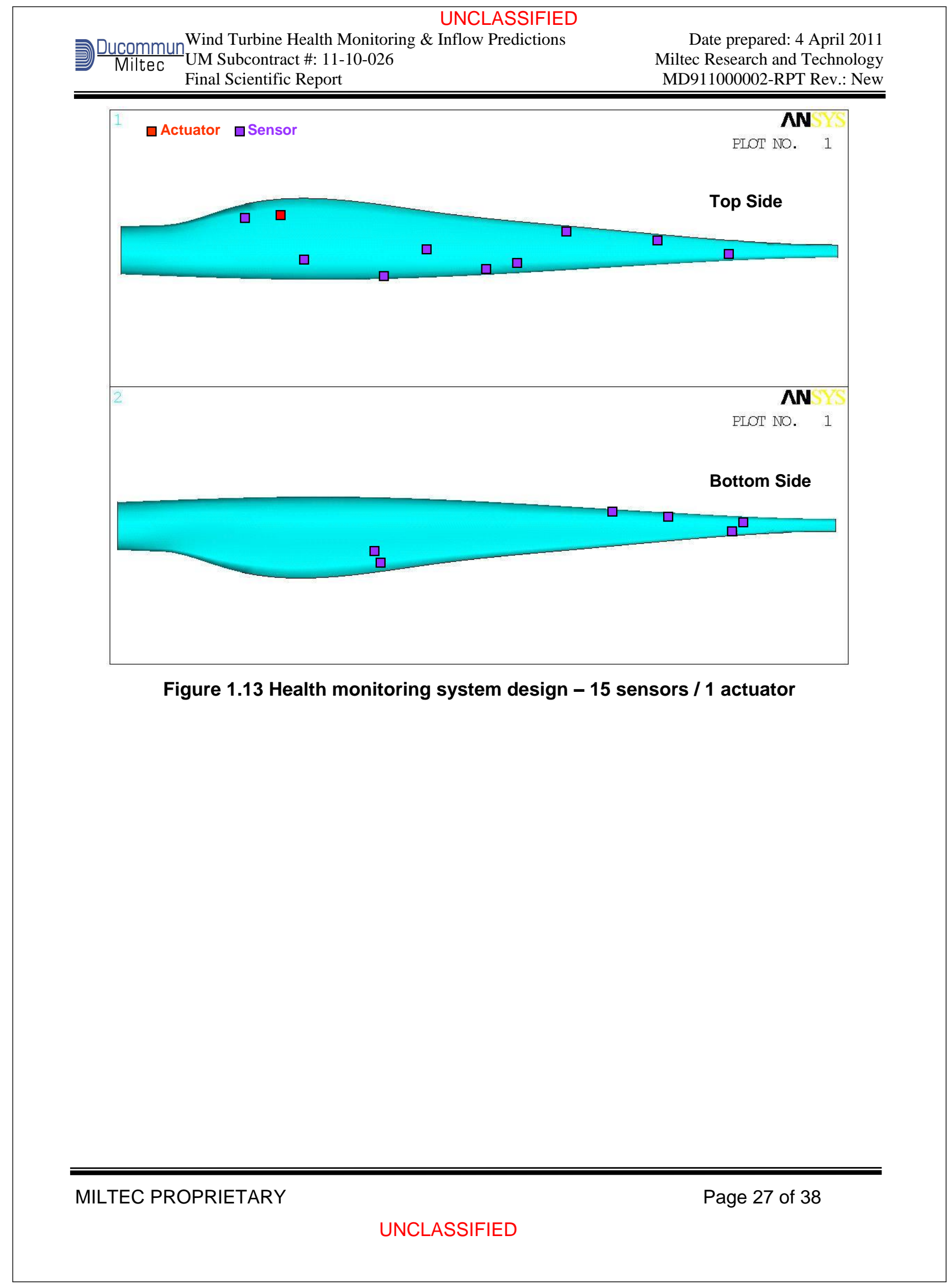




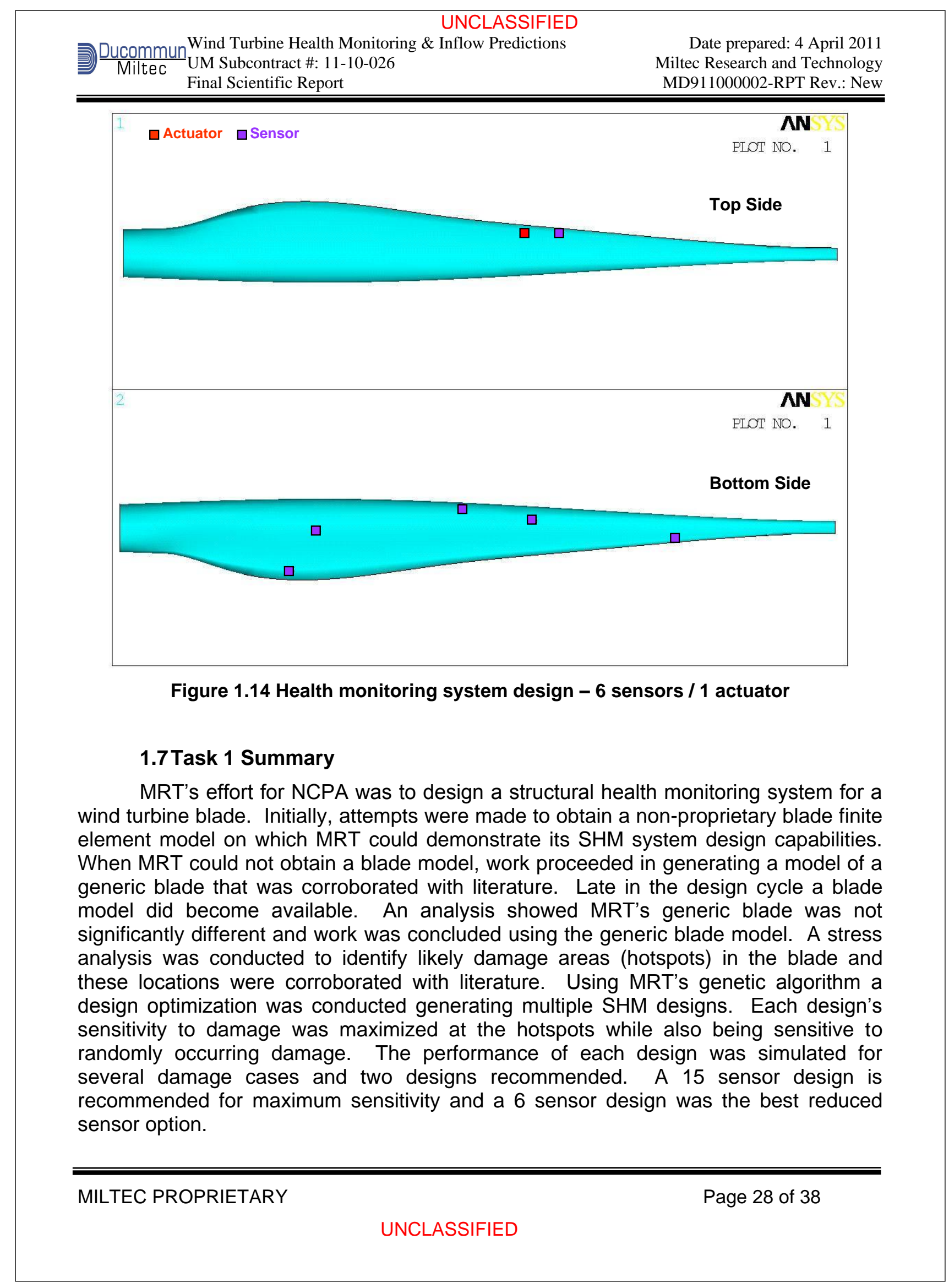




\subsection{Task 2 - Passive Detection of Wind Inflow Conditions}

A field data collection test was conducted during the week of November 8 through 12. The Miltec stand-alone point sensors were deployed in a wagon-wheel arrangement simultaneously with an 3-D sonic anemometer located in the center. The equipment was deployed in the field figure below, with GPS center $34.26453^{\circ} \mathrm{N}$, $90.20182^{\circ} \mathrm{W}$. This site is near Locke Station and is a flat, open field.

The stand-alone sensor DAQ provides data at $2000 \mathrm{~Hz}$, with the sensor response being flat (within $3 \mathrm{~dB}$ ) from 0.025 to $100 \mathrm{~Hz}$.

The anemometer height was 124 inches.

The aperture of the wagon was approximately $384 \mathrm{~m}$, and 49 point sensors were used.

A 5-channel (24-bit) datalogger sampled the anemometer and a B\&K 4193 low frequency microphone at $2048 \mathrm{~Hz}$. The microphone was used for baseline comparison testing, but was only used for a short time beginning 20:15 UTC on Tuesday, November 9.

Known signals occurred at the following specified time intervals:

\section{Table 2.1}

Date

$11 / 8 / 2010$

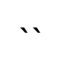



$\cdots$

.

$\cdots$

,

$11 / 8 / 2010$

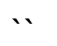

$\because$

, n

․ n

,

ㄴ

11/9/2010 Airplane

. Propane cannon

-
Source

Propane cannon

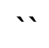

․

$\because$

“

?

?

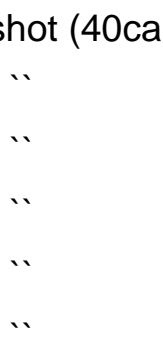

.
Time (UTC)

22:15:21

22:15:22

22:16:11

22:16:12

22:17:54

22:17:55

22:17:56

22:23:50

22:23:51

22:23:52

22:23:53

22:23:54

22:23:55

20:56:??

$21: 22: ? ?$

21:22:??

\section{GPS location}

$34.25937 \mathrm{~N}, 90.20491 \mathrm{~W}$
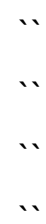

、

$\cdots$$$
\text { 、 }
$$$$
\cdots
$$$$
\cdots
$$$$
\text { 、 }
$$$$
\text { ․ }
$$$$
\cdots
$$

??

Prime has this value

'.

MILTEC PROPRIETARY

Page 29 of 38

\section{UNCLASSIFIED}


UNCLASSIFIED

Ducommun Wind Turbine Health Monitoring \& Inflow Predictions

Miltec UM Subcontract \#: 11-10-026

Final Scientific Report
Date prepared: 4 April 2011 Miltec Research and Technology MD911000002-RPT Rev.: New

\begin{tabular}{clcc}
\hline \hline$\cdots$ & restarted FP & $21: 48: 57$ & N/A \\
$11 / 10 / 2010$ & changed card \#40 & $19: 33: 59$ & N/A \\
$\cdots$ & changed card \#49 & $19: 38: 40$ & N/A \\
$\cdots$ & Cotton compressor & $20: 34: ? ?$ & $\sim 170$ from mag. N \\
$11 / 11 / 2010$ & Miltec drove up & $19: 0 ?: ? ?$ & $\sim 200$ from mag. N \\
. & airplane & $19: 18: ? ?$ & SE to SW
\end{tabular}

The array was setup inside the highlighted box below.

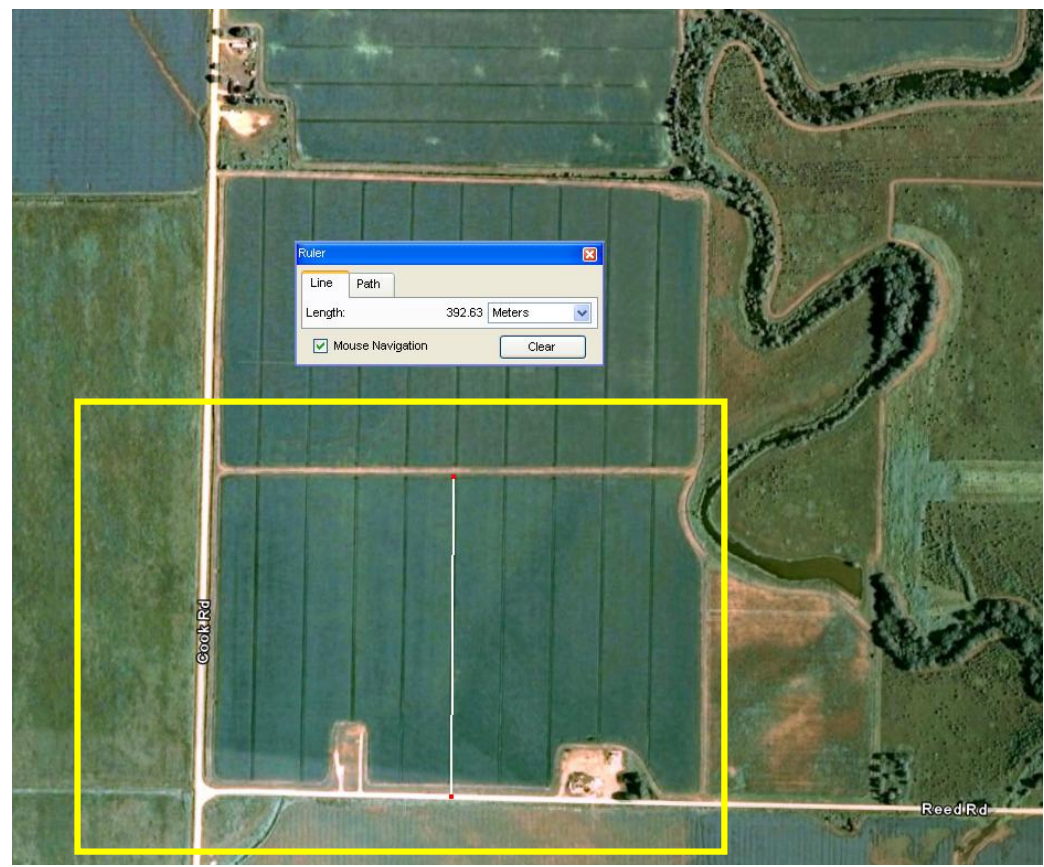

Figure 2.1 Modified Google Earth representation of the setup location.

The diagram below describes the approximate wagon wheel arrangement. The angle degrees are measured from Magnetic North, and the distance out from center in the legend is in meters. 


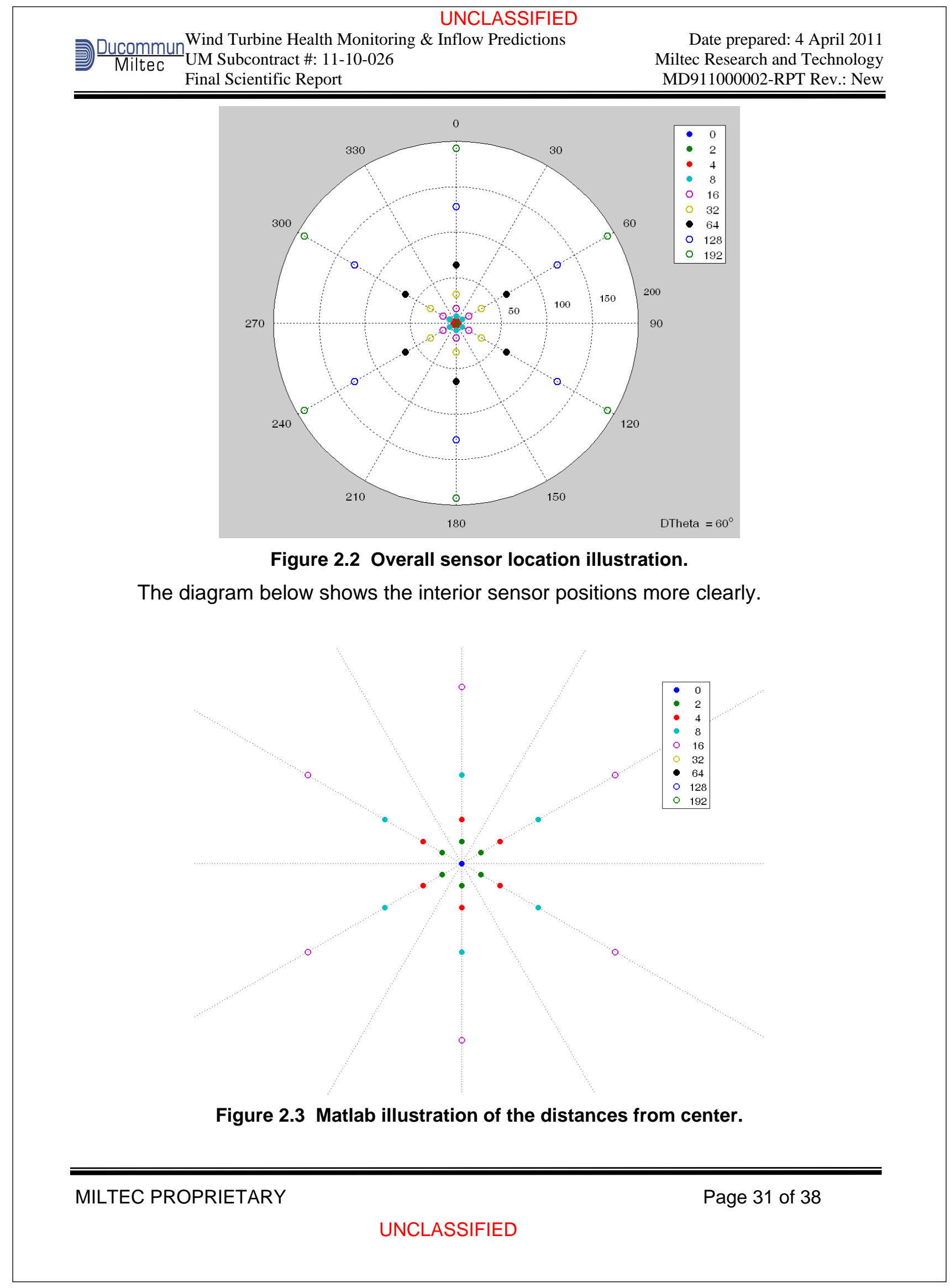

Page D-32

Final Report | DOE Award No. DE-EE0003269 
UNCLASSIFIED

Ducommun Wind Turbine Health Monitoring \& Inflow Predictions

Final Scientific Report
Date prepared: 4 April 2011 Miltec Research and Technology MD911000002-RPT Rev.: New

The sensors were setup in the following order:

\section{Table 2.2}

\begin{tabular}{lcl} 
Leg \# & $\begin{array}{c}\text { Leg Degrees from N } \\
\text { Center }\end{array}$ & \multicolumn{1}{c}{ Sensors (in to out) } \\
0 & 0 & 49 \\
1 & 60 & $1,2,3,4,5,6,7,8$ \\
2 & 120 & $9,10,11,12,13,14,15,16$, \\
3 & 180 & $17,18,19,00,21,22,23,24$ \\
4 & $25,26,27,28,29,30,31,32$ \\
5 & 240 & $33,34,35,36,37,38,39,40$ \\
6 & 300 & $41,42,43,44,45,46,47,48$
\end{tabular}

A rodent had chewed through the sensor cable of TT-040 on the night of $11 / 8 / 2010$. Thus, there will be no pressure data available on this unit from the break until the time of the repair. The unit was repaired on Tuesday, 11/9/2010, and the TT040 data was sampling pressure data by 20:48:?? UTC.

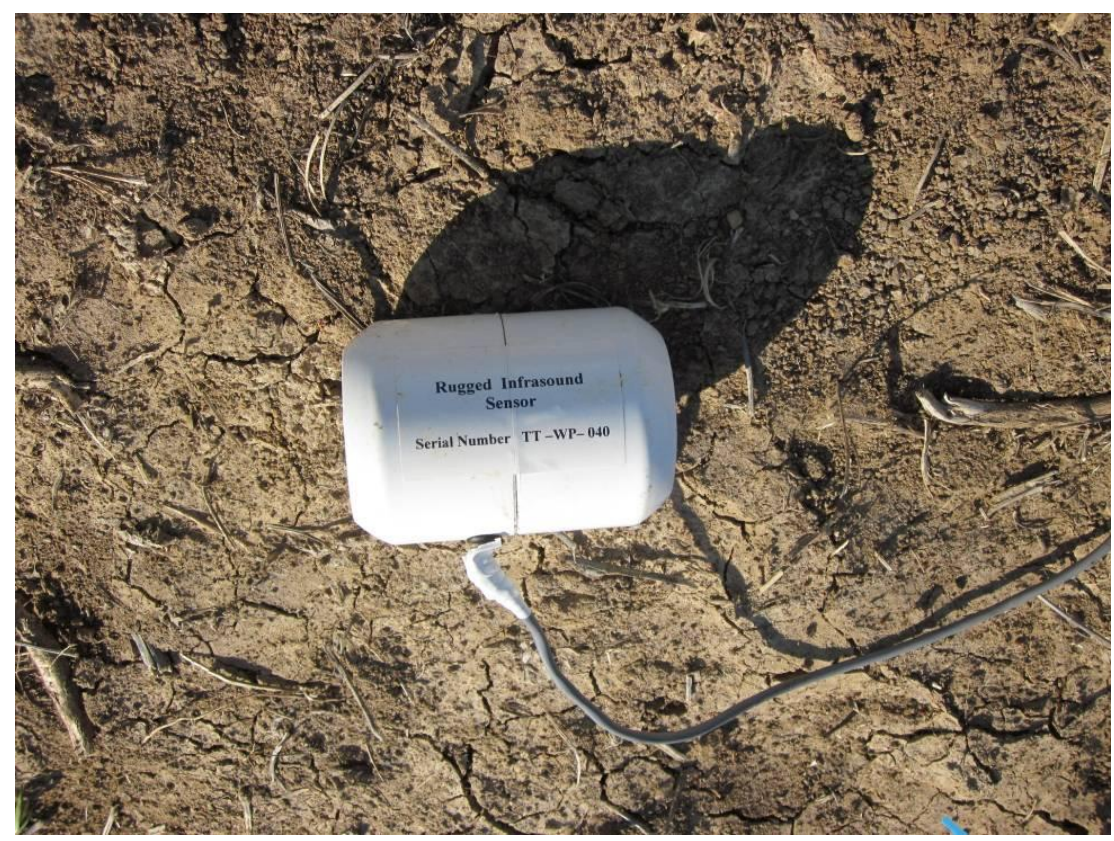

Figure 2.4 Repair after rodent attack. 


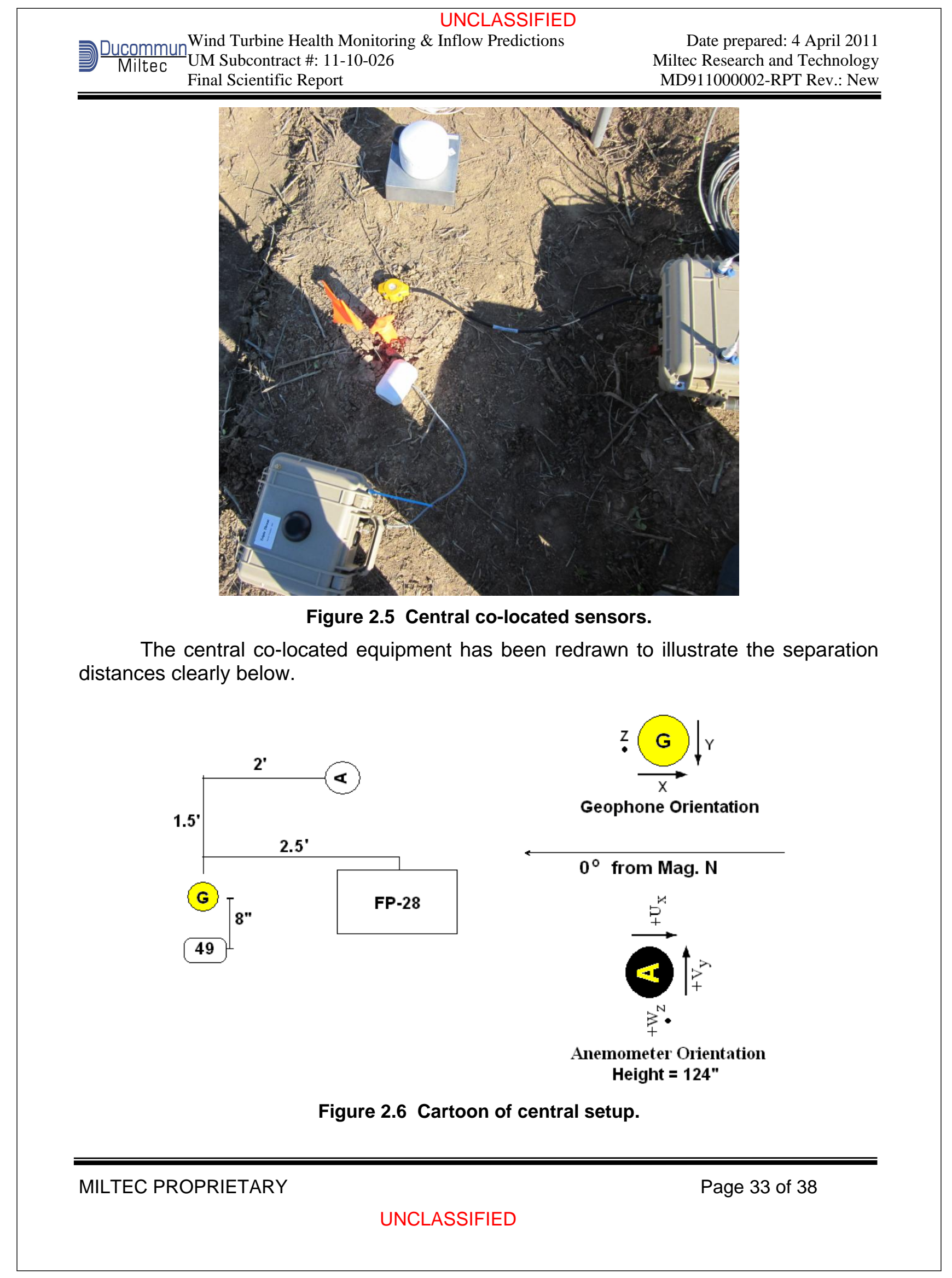




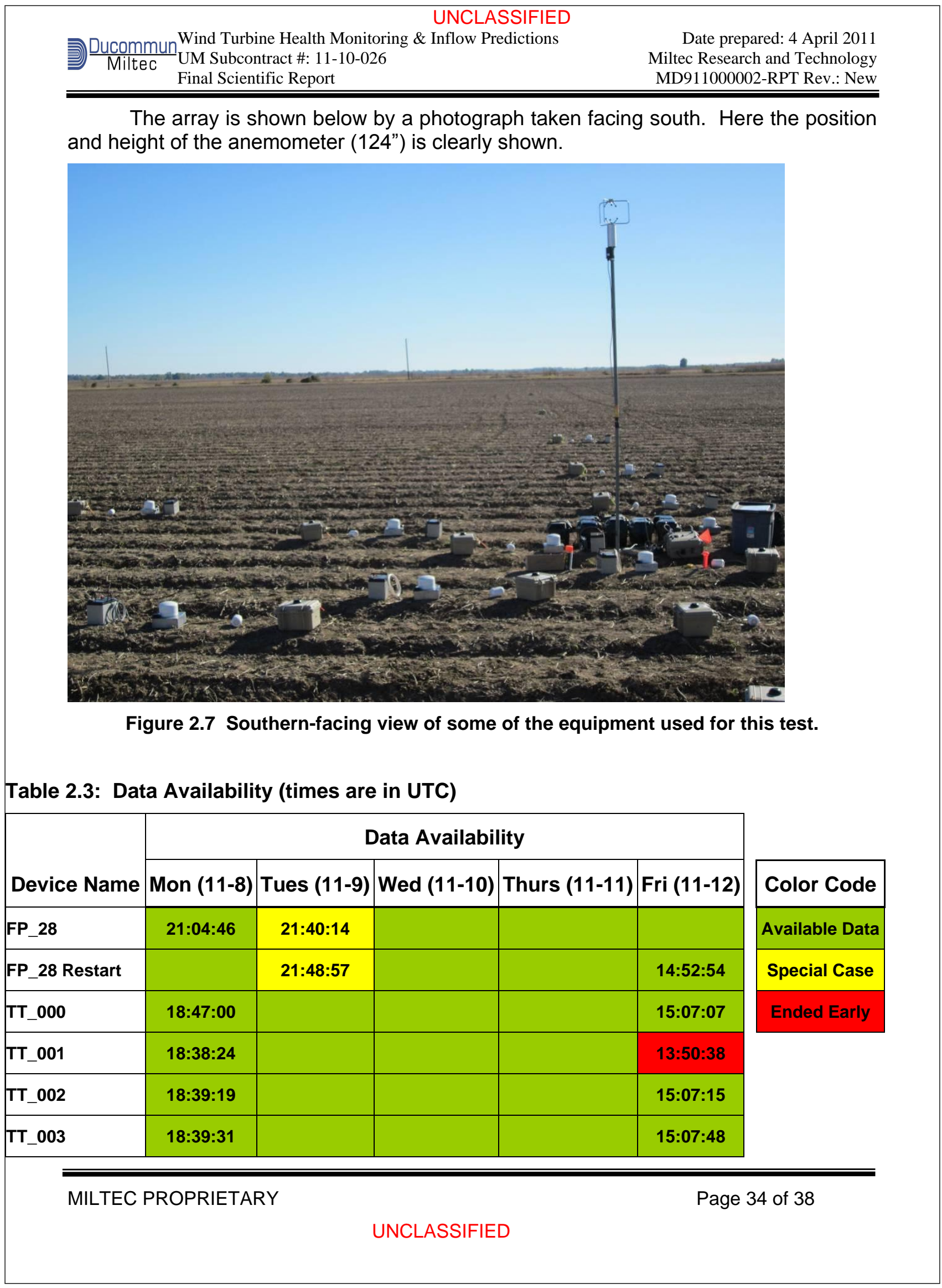


Appendix D | Miltec Research \& Technology - Final Report

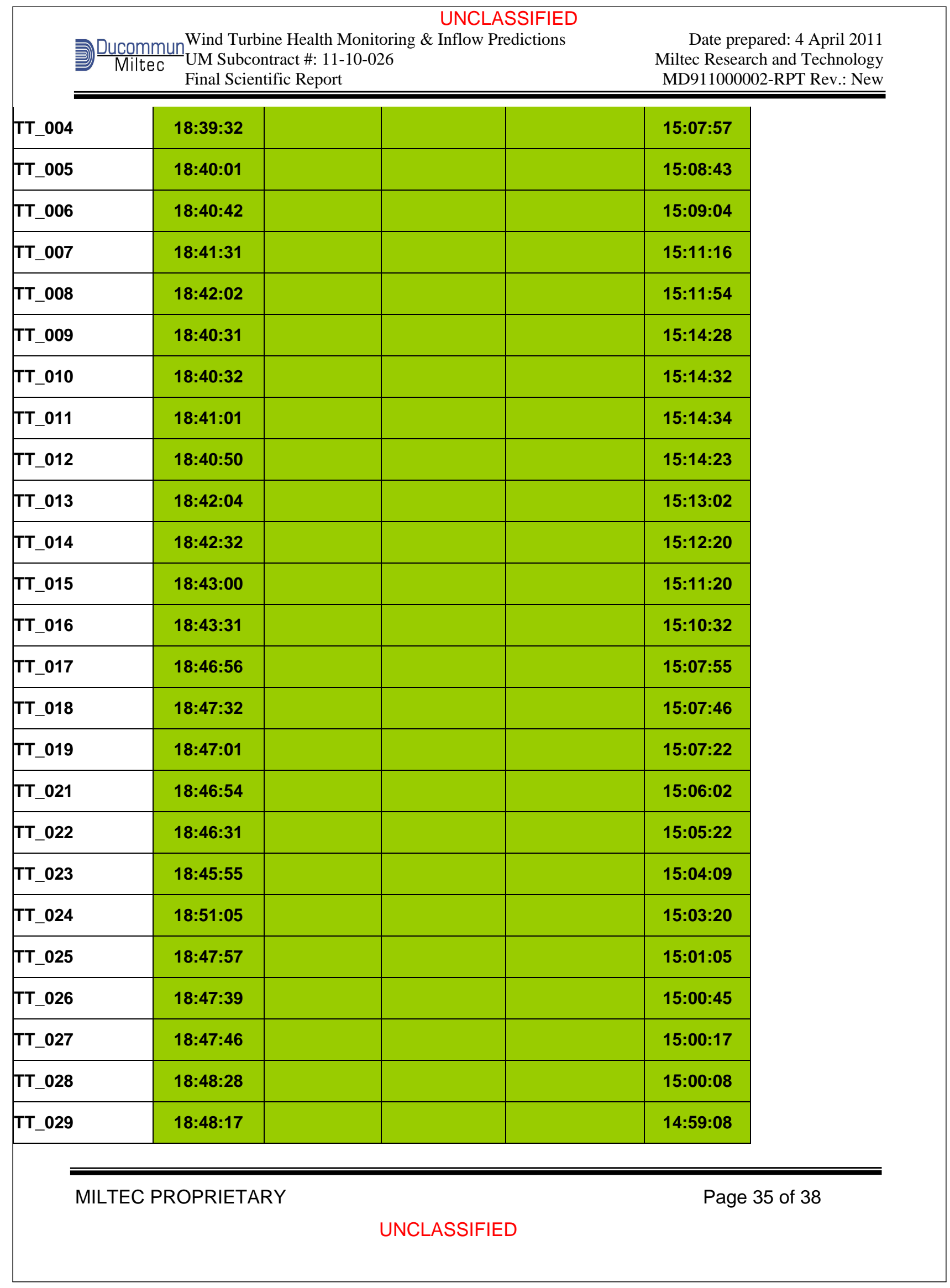


Appendix D | Miltec Research \& Technology - Final Report

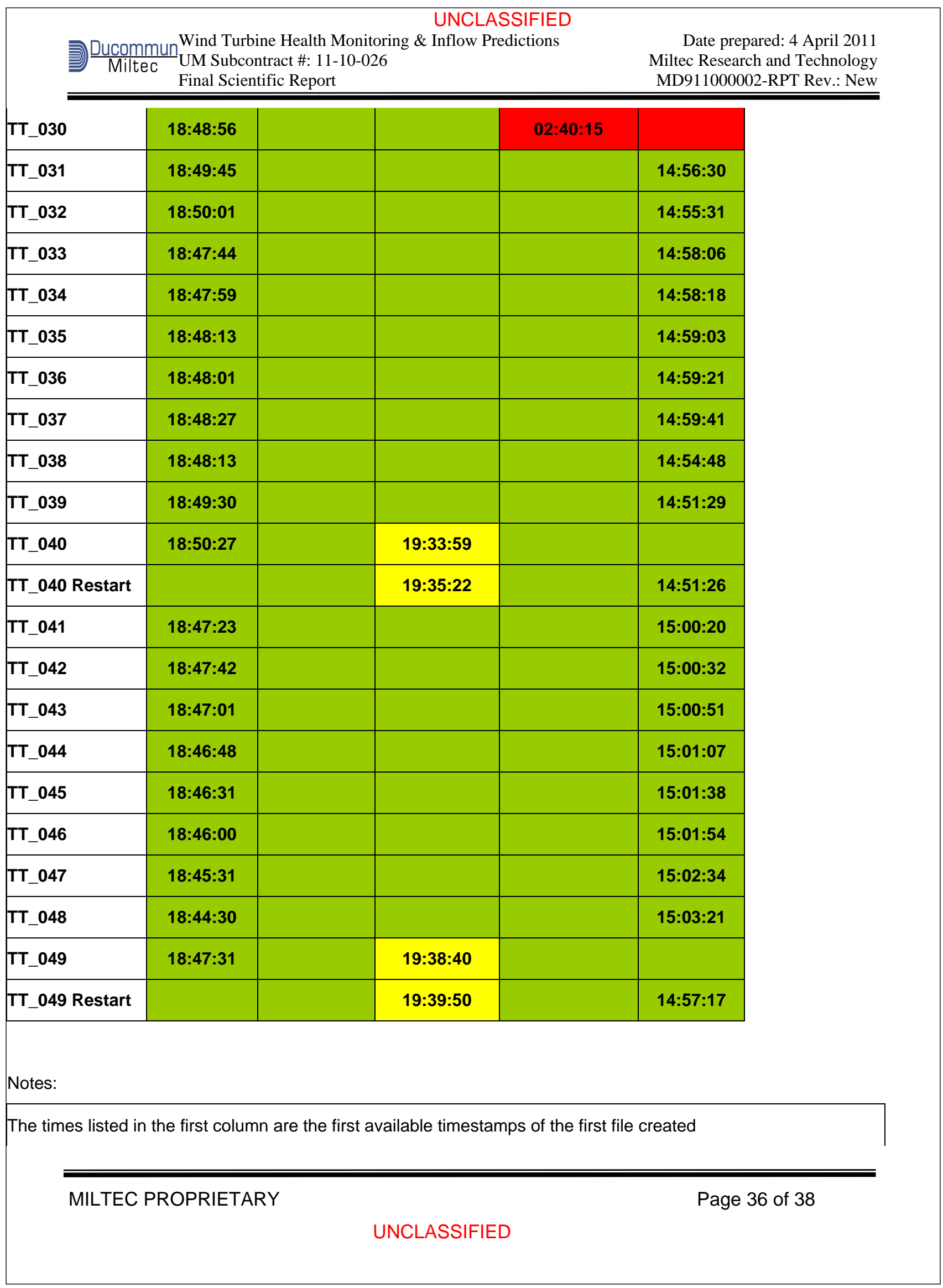




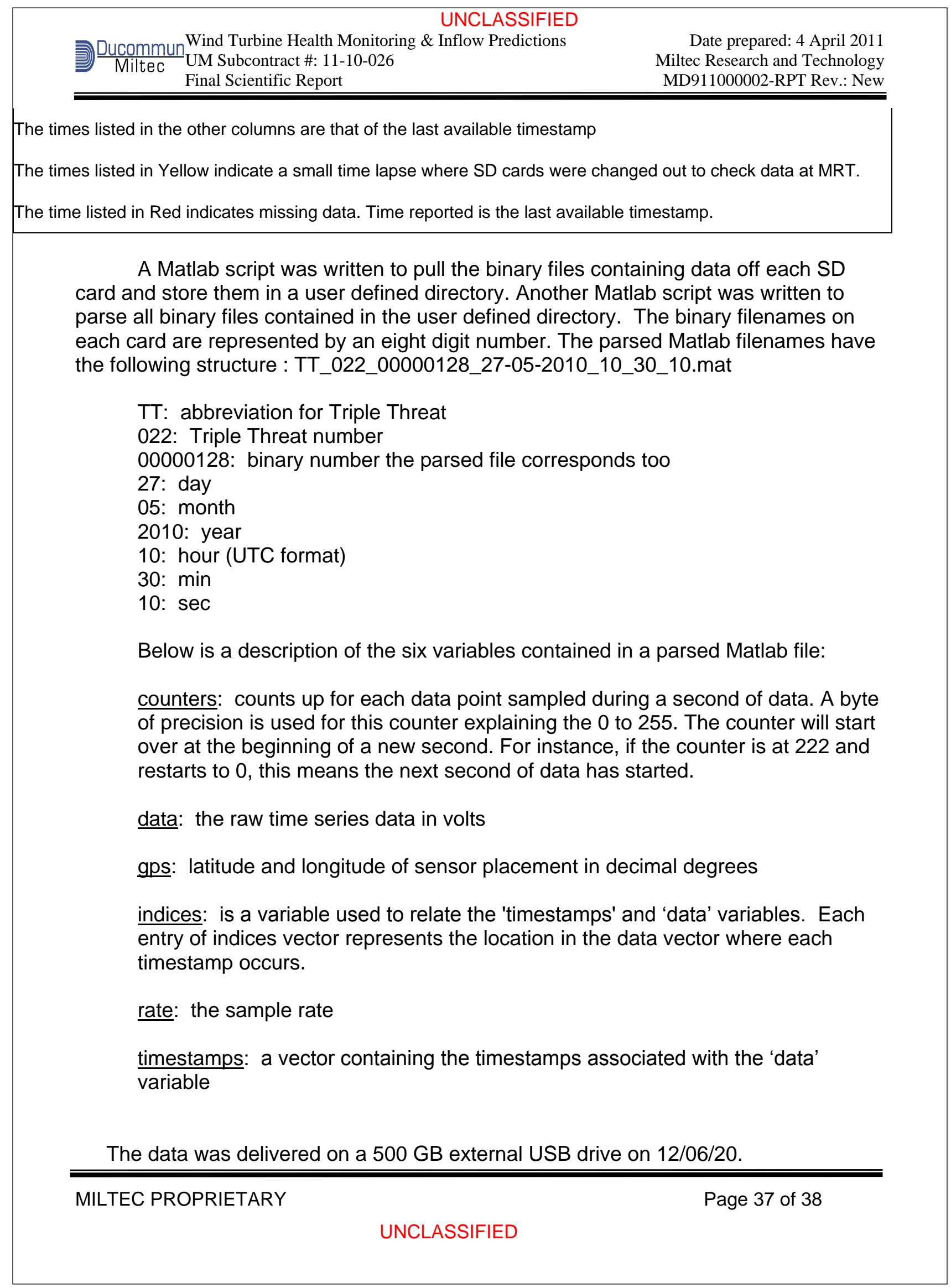




\subsection{References}

[1] Paquette, J., Laird, D., Griffith, D., and Rip, L., "Modeling and Testing of 9m Research Blades," AIAA-2006-1199, 44 ${ }^{\text {th }}$ AIAA Aerospace Sciences Meeting and Exhibit, Reno, NV, Jan. 9-12, 2006.

[2] Resor, B. and Bushnell, T., "A 1.5 MW NuMAD Blade Model," Draft Report, Sandia National Laboratories, Albuquerque, NM.

[3] Locke, J. and Valencia, U., "Design Studies for Twist-Coupled Wind Turbine Blades," SAND2004-0522, Sandia National Laboratories, Albuquerque, NM, June 2004.

[4] Burton, T., Sharpe, D., Jenkins, N., and Bossanyi, E., Wind Energy Handbook, John Wiley \& Sons, 2001.

[5] http://www.gurit.com/core/core_picker/download.asp?documenttable=libraryfiles $\& \mathrm{id}=687$

[6] http://www.diabgroup.com/europe/literature/e_pdf_files/ds_pdf/PB_DS.pdf

[7] Paquette, J., Dam, J., and Hughes, S., "Structural Testing of 9m Carbon Fiber Wind Turbine Research Blades," AIAA-2007-816, 45 ${ }^{\text {th }}$ AIAA Aerospace Sciences Meeting and Exhibit, Reno, NV, Jan. 8-11, 2007.

[8] Rumsey, M., Paquette, J., White, R., Werlink, R., Beattie, A., Pitchford, C., and Dam, J., "Experimental Results of Structural Health Monitoring of Wind Turbine Blades," AIAA-2008-1348, 46 $6^{\text {th }}$ AIAA Aerospace Sciences Meeting and Exhibit, Reno, NV, Jan. 7-10, 2008. 